\section{$\circ$ OCEAN BOTTOM SEISMIC SCATTERING}

by

Martin Eugene Dougherty

B.S., Winona State University (1983)

SUBMITTED IN PARTIAL FULFILLMENT OF THE

REQUIREMENTS FOR THE DEGREE OF

DOCTOR OF PHILOSOPHY

at the

MASSACHUSETTS INSTITUTE OF TECHNOLOGY

and the

WOODS HOLE OCEANOGRAPHIC INSTITUTION

$\frac{\overline{0}}{\frac{1}{3}}$

\author{
August, 1989 \\ copyright \\ Martin E. Dougherty, 1989
}

The author hereby grants to MIT and WHOI permission to reproduce and distribute copies of this thesis document in whole or in part.
7.1

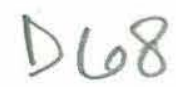

1989

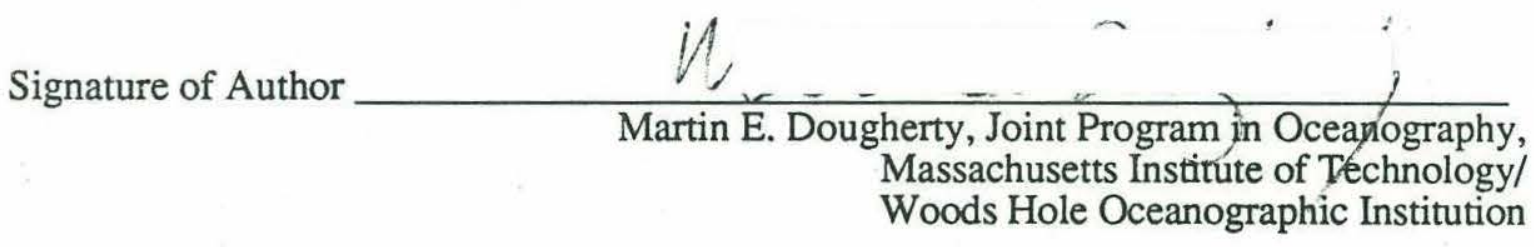

Certified by

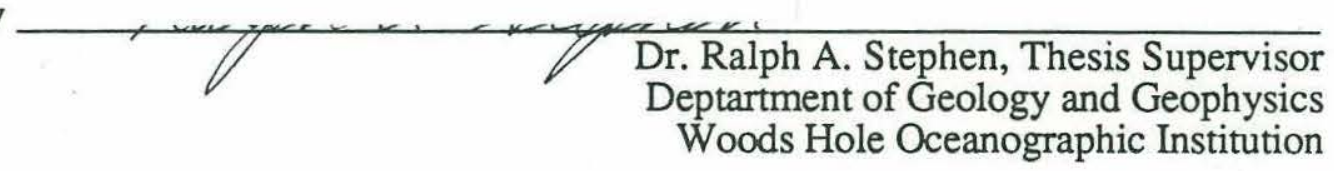

Accepted by

Dr. G.P. Lohmann, Chair

Joint Committee for Geology and Geophysics

Massachusetts Institute of Technology/

Woods Hole Oceanographic Institution

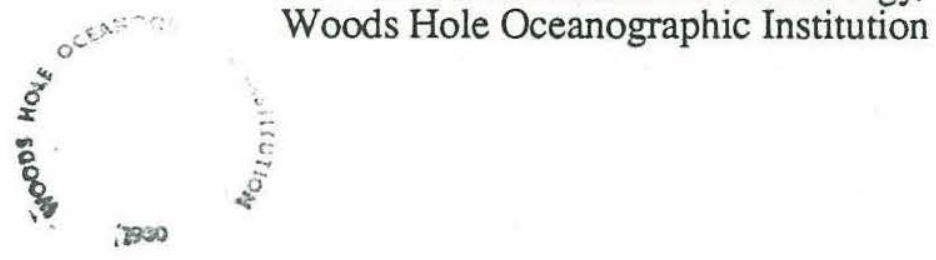


HAM

0.101 영

(igA

di.

worlegoon

II W 


\title{
OCEAN BOTTOM SEISMIC SCATTERING
}

\author{
by \\ Martin Eugene Dougherty
SUBMITTED IN PARTIAL FULFILLMENT OF THE REQUIREMENTS FOR THE DEGREE OF
DOCTOR OF PHILOSOPHY \\ at the \\ MASSACHUSETTS INSTITUTE OF TECHNOLOGY \\ and the \\ WOODS HOLE OCEANOGRAPHIC INSTITUTION \\ August, 1989
}

\begin{abstract}
Seismic studies of the oceanic crust, both experimental and theoretical, often assume a flat seafloor and laterally homogeneous crust. This is done regardless of the appearance in seismic data of obvious effects due to scattering from lateral heterogeneities both on and in the seafloor. Detailed fine scale surveys of mid-ocean ridges, where the upper oceanic crust is exposed, have revealed the presence of lateral heterogeneities in the form of complicated topography, extrusive volcanic structure, and abundant fracturing and faulting. These heterogeneities have a significant affect on the propagation of seismo/acoustic energy through the crust, especially in the immediate vicinity of the seafloor. This thesis deals with the problem of scattering of seismo/acoustic energy from a number of forms of lateral heterogeneity in the upper oceanic crust.

A common theme throughout this work is that the size of the heterogeneity on or in the seafloor is of the same order of magnitude as the seismo/acoustic wavelength. This is the realm of scattering theory where the wave-like characteristics of seismic energy have a particularly large influence on the outcome of interaction with structure in the media. The work presented here involves the application of the finite difference modeling technique to problems concerning laterally heterogeneous elastic media. This method is a full wave solution to the elastic wave equation and as such includes all wave interactions with the media. The finite difference formulation is used to study four distinct phenomena; scattering from discrete deterministic seafloor features; wave propagation through continuous randomly heterogeneous upper oceanic crust; scattering from more complicated topographic profiles and the limitations of the method for the rough seafloor problem; and the problem of plane acoustic wave scattering from an infinite elastic cylinder.

The principal finding of this work is that lateral heterogeneities in the upper oceanic crust can have a dramatic affect on seismo/acoustic wave propagation. Scattering from rough seafloors and/or volume heterogeneities is often quite similar and causes the occurrence of signal generated 'noise' (coda), decorrelation of primary arrivals, and anomalies in arrival travel time and amplitude. Topographic and volume scatterers acting as secondary sources of seismic energy can cause a resonant coupling of body wave energy into interface (Stoneley) waves at the seafloor. This is possibly one mechanism by which natural seismic and storm generated acoustic energy can be coupled into seafloor noise.
\end{abstract}


The applicability of the use of the finite difference method for non-planar watersolid interfaces is also discussed. Models were calculated which approximate sinusoidal seafloors and plane acoustic wave scattering from an infinite elastic cylinder. The discretization of a rectangular difference grid must be extremely fine to accurately accommodate a smoothly varying water-solid interface which does not align with the grid. Regardless of the discretization concerns, the rough seafloor models presented here demonstrate the arrivals expected from larger scale sinusoidal topography as well as the importance of considering quite small ( $<1 / 15$ wavelength) topographic features in the scattering problem. Also, steep topography will allow seismo/acoustic energy to enter the seafloor at very large ranges because the angle of incidence can repeatedly fall below the critical angle for transmitted energy, especially for converted shear energy. Ray theoretical shadow zones do not occur in these models (or in the real world) because of Franz-type waves diffracting into areas where the grazing angle is less than zero. 


\section{TABLE of CONTENTS}

\section{PAGE}

ABSTRACT

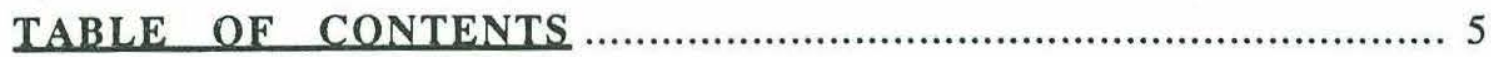

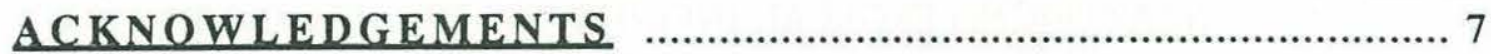

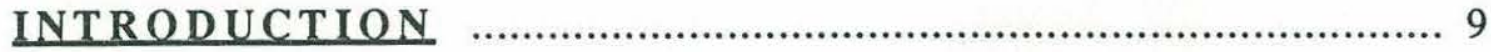

References ….................................................... 17

CHAPTER 1. GEOACOUSTIC SCATTERING FROM SEAFLOOR

FEATURES IN THE ROSE AREA ………….................. 21

Abstract …........................................................ 23

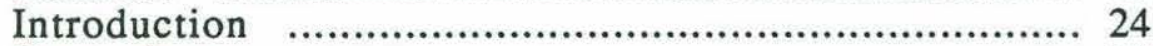

Data Area ....................................................... 30

Travel Time Analysis ……......................................... 33

Finite Difference Modeling ……..................................... 43

A Flat Model …................................................. 45

Diffraction Models ....................................................... 60

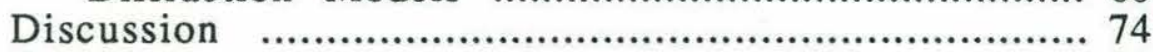

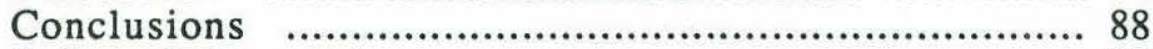

Acknowledgements …................................................ 90

References ........................................................ 91

CHAPTER 2, SEISMIC ENERGY PARTIONING AND SCATTERING

IN LATERALLY HETEROGENEOUS OCEAN CRUST ......... 93

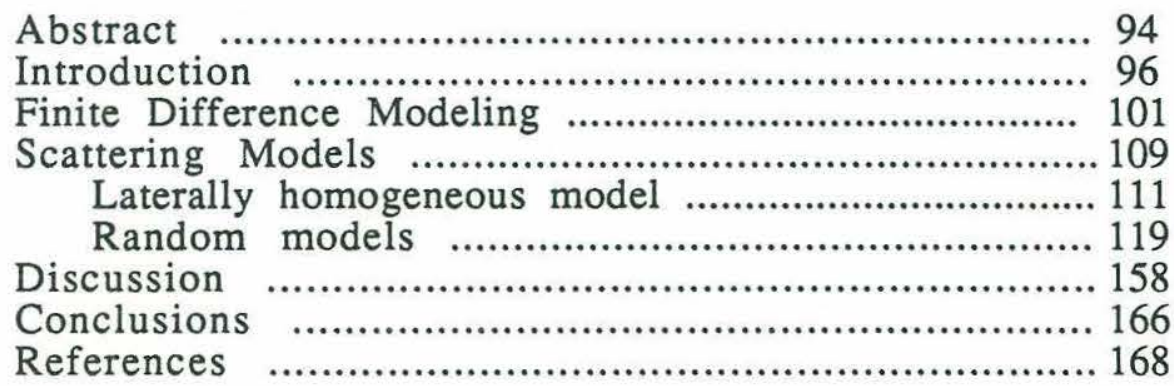


CHAPTER 3.SEISMO/ACOUSTIC PROPAGATION THROUGH

ROUGH SEAFLOORS ….......................................... 171

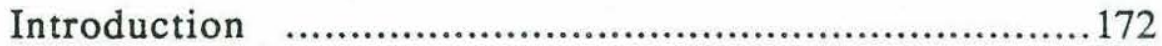

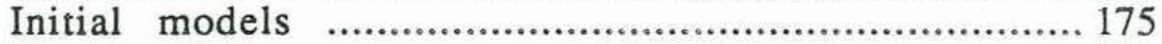

Test models ..................................................... 231

Finer grids ..............................................231

Reciprocal problem .........................................239

Conclusions ...................................................... 251

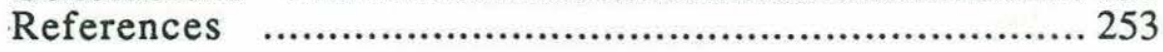

CHAPTER 4, A TIME DOMAIN SOLUTION TO ACOUSTIC WAVE

SCATTERING FROM AN INFINITE ELASTIC CYLINDER .. 257

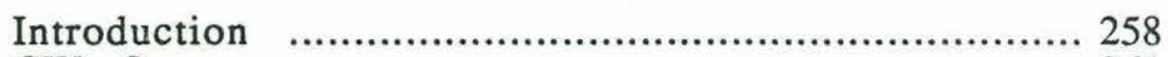

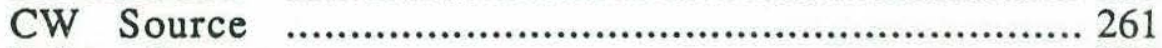

Pulse Source ..........................................................2 281

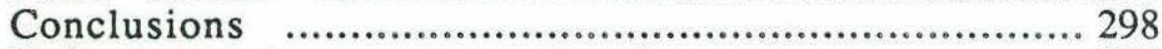

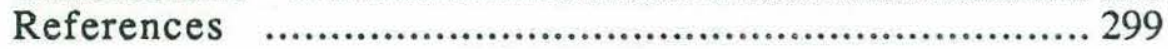

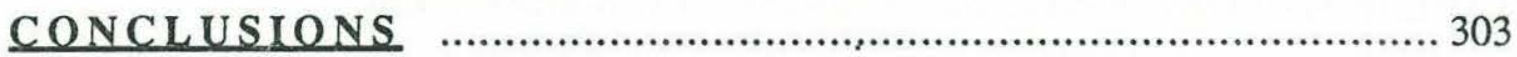




\section{ACKNOWLEDGEMENTS}

I have always felt that life was made easy by the values which I learned as a child. The influence of my parents, Boyce and Shirley Dougherty, on all aspects of my life cannot be overstated. I wish to thank them both for their love, foresight, and the sacrifices they made over the years for their children. My wife, Robin Coen, has been a constant companion throughout my varied career as a student, although sometimes from a great distance. Through many years of spousal unemployment, Robin has remained immeasurably tolerant and loving. Beecher Wooding graciously provided friendship, perspective, and daily wind reports. I look forward to many future rendezvous with Chris and Beecher at exotic sailing destinations around the world.

My thesis advisor, Ralph Stephen, has been unswerving in his support, both moral and financial. I am especially appreciative of the more recent help that Ralph has given me to become independently established in the post-graduation research world, even though sometimes the competition will undoubtedly be between ourselves. My hope is that Ralph will look back on his first experience of being a thesis advisor as a successful one. Tom Bolmer, Steve Swift, and more recently, Dan Burns helped make daily life in front of the terminal much more tolerable. Mary Hunt, Warren Sass, Andy Maffei, and the rest of the IPCL crew probably saved me years of computer hassles and put up with a lot of stupid questions and unreasonable requests.

Barry Parsons at MIT and Dick von Herzen at WHOI supported me in my first few years of the Joint Program. The staff at both the MIT and WHOI Joint Program offices, particularly Jake Peirson, Abbie Jackson, and Mary Athanis, make life too easy for all of the students, even those who complain too much. 
This work has been supported at various times by the Office of Navel Research (contract \#N00014-85-C-0001), the National Science Foundation (contract \#OCE8761132 and the Division of Advanced Scientific Computing) and the John von Neumann National Supercomputer Center. 


\section{INTRODUCTION}

The ultimate objective of much of the work in the fields of marine geology and geophysics is to understand the structure and processes of evolution of the oceanic crust. Marine seismology is the science of the use of sound wave energy (both acoustic and seismic) to remotely examine the structure of the ocean subbottom. While the exact nature of the relationship between seismic velocity, impedance, and lithology is still under investigation (Karson and Fox, 1986; Lewis, 1983; Spudich and Orcutt, 1980; Spudich and Orcutt, 1980), there is no doubt that the results of marine seismic experiments have told us much about the gross structure of the oceanic crust. Inherent in the use of seismology as a tool is the assumption of a basic understanding of the physics of wave propagation through the medium in question. The physics of seismic wave propagation through elastic media have been used in various states of approximation to model seismic data collected in the field. This thesis involves the use of the finite difference modeling technique to study wave propagation through laterally heterogeneous upper oceanic crust.

The classical picture of the oceanic crust is one of a discrete number of flat-lying homogeneous layers with fairly predictable velocities which correlate with age (Houtz and Ewing, 1976). This layered structure is generally composed, in descending order, of sediments, extrusive basalts, intrusive gabbroic dikes, and a peridotitic mantle at a depth of

5-7 kilometers below the sediment-basement interface. Simple seismic models using velocities assigned to these layers were sufficient to reproduce primary wave travel times of early seismic experiments. More accurate modeling techniques and better controlled marine seismic experiments have since shown that the classical oceanic structure, while close to 
correct 'on average', does not explain the lack of inter-crustal reflections and the amplitude of refracted arrivals seen in the field data (Spudich and Orcutt, 1980). Rather, the 'layers' of oceanic crust are now delineated as areas of crust with fairly constant vertical velocity gradients (Collins, 1988; Spudich and Orcutt, 1980). More sophisticated modelling techniques can now reproduce travel times, amplitudes, and phase information seen in seismic data (Helmberger and Morris, 1970; Fuchs and Müller, 1971).

Seismo/acoustic modelling and state-of-the-art seismic experimental methods have traditionally driven each other to higher levels of accuracy. Although the vertical picture has changed, lateral homogeneity in the upper oceanic crust is still assumed today in most cases. This is due in part to the ability of 1-dimensional velocity structures to reproduce the gross features of seismograms, but is also due to the inability of most '2-dimensional' modelling schemes to handle truly 2-dimensional, or laterally heterogeneous, earth models. There are secondary features in virtually all seismic data which are due to scattering from some type of lateral velocity feature.

Another commonly held belief is that seismic methods cannot resolve structure which has lateral extent on the order of the seismic wavelength in size. This is true in the context of trying to resolve laterally homogeneous structure with vertical extent less that a wavelength in size. However, heterogeneities of this size have a dramatic effect on seismic wave propagation. In fact, scattering of primary energy reaches a maximum when $k a(\mathrm{k}=$ wavenumber $=2 \pi$ / wavelength, $a=$ heterogeneity length scale) is approximately equal to one (Aki and Richards, 1980). Scattering of energy from heterogeneities manifests itself mainly in secondary features of the seismograms such as signal generated 'noise' (coda), secondary body and interface waves, and signal decorrelation. If the heterogeneities are large enough, deterministic, predictable effects such as noticeable travel time and amplitude anomalies will also appear in the seismograms. 
This dissertation is a study of the effects of a lateraily heterogeneous upper oceanic crust on the scattering of seismo/acoustic energy both at and below the seafloor. The method of finite differences is used to model propagation effects in media with heterogeneity sizes on the same order of magnitude as the seismic wavelength. Chapters in this work cover a number of 'types' of lateral heterogeneity. Scattering from deterministic, isolated, seafloor features is investigated in Chapter 1. Chapter one appears here with permission from Journal of the Acoustical Society of America (Dougherty and Stephen, 1987). Crustal volume heterogeneities can also have a strong influence on propagation. Chapter 2 deals with these types of media and also appears here with permission from the Journal of Pure and Applied Geophysics (Dougherty and Stephen, 1988). Further investigation into the effects of more complicated topography is made in chapter 3. Chapter 4 is a comparison between the analytical and finite difference solutions to the problem of plane acoustic wave scattering from an infinite elastic cylinder.

Chapter one addresses the problem of deterministic scattering from distinct seafloor features. Seismic data often contain sections of relatively large anomalous arrivals superimposed on expected arrivals for small groups of traces. One line of airgun data from an ocean bottom hydrophone (OBH) in the Rivera Ocean Seismic Experiment (ROSE) contains a very coherent diffracted arrival just after the primary refracted wave. This arrival is referred to as a "refraction branch diffraction" to distinguish it from diffraction hyperbola of near normal incidence reflections. The finite difference method (Bhasavanija, 1983; Clayton and Engquist, 1977; Kelly et al., 1976; Nicoletis, 1981; Stephen, 1984) was used to demonstrate that there are no simple reflection paths which can account for the arrival. Rather, it is due to secondary scattering from any of a number of seafloor features with height about equal to the acoustic wavelength. Energy is scattered from the seafloor 
features both into the water column and back into the crust where it propagates to the receiver.

The interaction of seismo/acoustic energy with discrete scatterers is fairly simple conceptually when compared to the problem of propagation through a continuously varying medium. Random distributions of heterogeneity in the upper oceanic crust can account for much of the signal generated 'noise' often seen in marine seismic data. Also, decorrelation in the primary arrival waveforms is caused by interaction with strongly heterogeneous media. These effects are studied in chapter 2 by calculating the wave propagation through a number of marine models with random velocity perturbations containing a range of heterogeneity correlation lengths.

The theoretical study of wave propagation through media with continuous volume heterogeneities has been well developed. However, the solution to the problem of the influence of strong velocity variations with $k a$ near one remains to be a difficult one. Historically, most of the effort concerning scattering from volume heterogeneities has been directed at the amplitude attenuation and coda wave excitation of seismic energy from earthquakes or teleseismic explosive sources (Aki, 1973; Aki, 1982; Frankel and Clayton, 1986; McLaughlin, Anderson et al., 1985; McLaughlin, Johnson et al., 1983; Menke and Chen, 1984; Menke and Dubendorff, 1985; Sato, 1982; Wu, 1982; Wu, 1983; Wu and Aki, 1988). Large scale heterogeneities have the greatest influence on primary arrival amplitude attenuation while smaller scale heterogeneities seem to control coda wave frequency content and decay (Aki, 1973; Aki, 1982). Most analytical work done to explain these results depends on the validity of the Born approximation which only considers the first interaction of energy with a scatterer (no multiple scattering) and that the scattering is weak with respect to the primary wave amplitude. When the experiment is scaled to that of typical marine refraction work, the Born approximation is not necessarily valid (Hudson, 
1982; Hudson and Heritage, 1981). The case of scattering of vector waves from a point source travelling through a medium with velocity gradients, topography, and random heterogeneities has little chance to be solved analytically. Therefore, numerical methods are probably the most effective resource for studying this problem.. The finite difference method has already been applied to the problem for the case of laterally heterogeneous halfspaces with plane wave sources (Frankel and Clayton, 1986; McLaughlin et al., 1985; McLaughlin et al., 1983). However, these studies have not dealt with a pulse source travelling through the strong heterogeneities or the water-solid interface of the marine case. The marine case is particularly interesting because of the coupling of acoustic and seismic energy at the seafloor. Stephen has used this method successfully to study the scattering effects of simple lateral heterogeneity in the oceanic crust (Stephen, 1984a,b; 1985; 1988a,b,c; Stephen and Bolmer, 1985). The finite difference formulation used for the work in chapters 2 and 3 is a displacement-stress formulation and is well suited for use with the marine problem (Stephen, 1988; Virieux, 1984; Virieux, 1986).

In general, random scattered 'noise' in the seismograms and decorrelation of the primary compressional diving wave reaches a maximum for $k a$ near one. As $k a$ increases much above one, deterministic travel time and amplitude effects become more prevalent. As $k a$ decreases much below one, the medium becomes an equivalent homogeneous medium (Aki and Richards, 1980) and coherence of primary arrivals returns. Another interesting result of chapter 2 is that the heterogeneities near the water-solid interface can act as secondary sources of interface waves when energy is scattered from them. It has been proposed that seafloor noise propagates as some type of interface wave along the seafloor. However, the source for these interface waves was unknown. The work in chapter 2 implies that this 'noise' can be generated by secondary scattering of acoustic waves or microseisms from heterogeneities within the crust but near the water-solid interface. 
Chapter 3 involves more investigation of scattering form rough seafloors. The problem of seismic energy scattering from heterogeneities within the crust is complicated by the presence of topography on the seafloor or sediment/basement interface. While heterogeneities within the crust can contain velocity contrasts on the order of 1-20 per cent, the water-solid interface can represent a velocity contrast of over 100 per cent. Because of this sharp impedance contrast, non-planar seafloors can cause large scattering effects when energy propagates into the oceanic crust. With increased interest in the seismic study of mid-ocean ridges where the impedance contrast is particularly high, it it important to understand the relationship between topography and seismo/acoustic propagation through the crust.

As with the problem of wave propagation through volume heterogeneities, the rough surface scattering problem has also been very well studied. However, the approximations used for most of the known solutions make them inappropriate for the study of elastic scattering at realistic seafloors (Ogilvy, 1987). These solutions include Rayleigh's method of plane wave summation (Rayleigh, 1878), the method of small perturbations (Gilbert and Knopoff, 1960; very small topography with respect to acoustic wavelength), the Kirchhoff method (Eckart, 1953; assumes scattering is from tangent planes along the topography) and methods which sum contributions from distributions of point sources, facets, or protuberances along the bottom (see Ogilvy, 1987).

Methods which require fewer limiting assumptions and are more flexible have more recently been used to study this problem. Bouchon (1985), Campillo and Bouchon (1985), and Paul and Campillo (1988) have successfully used a boundary integral formulation coupled with the discrete wavenumber method to study diffractions from corrugated boundaries. The computationally intensive finite difference/element, and pseudospectral method (Kosloff, et al., 1984; Fornberg, 1986, 1988) have also become 
attractive because of the full solution to the problem of surface and subsurface scattering with very few initial limiting assumptions. Stephen (1984) and the work from chapter 1 of this work use the method of finite differences to explain scattering effects of discrete seafloor features on both sides of the critical range. Hill and Levander, (1984) and Levander and Hill (1985) also used the finite difference method to model scattering from rough low velocity layers within the crust and rough elastic surface layers.

In chapter 3 the finite difference method is used to model propagation through sinusoidal seafloors. While the initial intent of this work was to help to distinguish between volume and surface scattering, this chapter actually deals mainly with the question of the appropriateness of the use of the method for this type of problem. The principle problem with these models is in the approximation of a curved seafloor surface on a rectangular grid. A rectangular stair-stepped structure must be used to closely approximate a continuously sloping seafloor. In this way, a kind of secondary microroughness is imposed onto the larger scale sinusoidal topography. Even though the stair steps are quite small in relation to the seismic wavelength, significant diffractions still occur from the microroughness on the seafloor. Scattering phenomena due to this problem are discussed in chapter 3 as well as some 'real' effects which can be expected from sinusoidal topography.

The problem of plane acoustic wave scattering from an infinite elastic cylinder is solved in chapter 4 with the finite difference formulation used in the work of chapters 2 and 3. The intent of this work was not to shed any new light on the solution to the problem, but rather, to use this well studied problem with exact analytical solutions as a rigorous test of the method and formulation. Work on the cylinder problem pointed out a slight asymmetry in the coding of the definition of elastic parameters on the finite difference grid for the displacement-stress formulation. The coding mistake was corrected and symmetry 
of the solution was established. All of the models in chapter 2 and the preliminary models of chapter 3 were calculated before this slight asymmetry in the grid was discovered.

Fortunately, the effect is a very small one and test reruns of some of these models with the corrected grid definition showed no measurable difference in model results discussed in the text. The TEST models presented in chapter 3 and all of the models presented in chapter 4 were run with the corrected grid definition. 


\section{References}

Aki, K., (1973), Scattering of $P$ waves under Montana LASA, J. Geophys. Res., 78, 1334-1346.

Aki, K., (1982), Scattering and attenuation, Bull. Seis. Soc. Am., 72, 5319-5330.

Aki, K. and Richards, P. G., (1980), Ouantitative Seismology, Theory and Methods, San Francisco, W.H. Freeman and Co.

Bhasavanija, K., (1983), A finite difference model of an acoustic loggin tool: The borehole in a horizontally layered geologic medium, $\mathrm{Ph}$. D. thesis, Colorado School of Mines, Golden, Colorado.

Clayton, R. and Engquist, B., (1977), Absorbing boundary conditions for acoustic and elastic wave equations, Bull. Seis. Soc. Am., 67, 1529-1540.

Collins, J. A., (1988), A search for layering in the oceanic crust, $\mathrm{Ph}$. D. thesis, Massachusetts Institute of Technology/Woods Hole Oceanographic Institution, Woods Hole, Massachusetts.

Dougherty, M. E. and Stephen, R. A., (1987), Geoacoustic scattering from seafloor features in the ROSE area, J. Acoust. Soc. Am., 82(1), 238-256.

Dougherty, M. E. and Stephen, R. A., (1988), Seismic energy partitioning and scattering in laterally heterogeneous ocean crust, PAGEOPH, 128(1/2), 195-229.

Eckart, C., (1953), The scattering of sound from the sea surface, J. Acoust. Soc. Amer.,v. 25 , p. $566-570$.

Fornberg, B., (1987), The pseudospectral method: Comparisons with finite differences for the elastic wave equation, Geophysics, 52, 483-501.

Fornberg, B., (1988), The pseudospectral method: Accurate representation of interfaces in elastic wave calculations, Geophysics, 53(5), 625-637.

Frankel, A. and Clayton, R. W., (1986), Finite difference simulations of seismic scattering: Implications for the propagation of short period seismic waves in the crust and models of crustal heterogeneity, J. Geophys. Res., 91, 6465-6489.

Fuchs,K. and Müller, G., (1971), Computation of synthetic seismograms with the reflectivity method and comparison with observations, Geophys. J. R. astr. Soc., v.23, p.417-433.

Gilbert, F. and Knopoff, L., (1960), Seismic scattering from topographic irregularities, J. Geophys. Res., v. 65, p. 3437-3444. 
Helmberger, D.V., and Morris, G.B., (1970), A travel time and amplitude interpretation of a marine refraction profile: Transformed shear waves, Bull. Seism. Soc. Am., v.60, p.593-600.

Houtz, R. E. and Ewing, J., (1976), Upper crustal structure as a function of plate age, J. Geophys. Res., 81, 2490-2498.

Hudson, J. A., (1982), Use of stochastic models in seismology, Geophys. J. Roy. Astr. Soc., 69, 649-657.

Hudson, J. A. and Heritage, J. R., (1981), The use of the Born approximation in seismic scattering problems, Geophys. J. Roy. Astr. Soc., 66, 221-240.

Karson, J. A. and Fox, P. J., (1986), Geological and geophysical investigations of the Mid-Cayman Spreading Centre: seismic velocity measurements and implications for the constitution of layer 3, Geophys. J. Roy. astr. Soc., 85, 389-411.

Kelly, K. R., Ward, R. W., Treitel, Sven, and Alford, R. M., (1976), Synthetic seismograms: A finite difference approach, Geophysics, 41, 2-27.

Kosloff, D., Reshef, M., and Loewenthal, D., (1984), Elastic wave calculations by the Fourier method, Bull. Seis. Soc. Am., 74, 875-891.

Lewis, B. T. R., (1983), The processes of formation of ocean crust, Science, 220, 151157.

McLaughlin, K. L., Anderson, L. M., and Der, Z. A., (1985), Investigation of scattering and attenuation of seismic waves using 2-dimensional finite difference calculations,Symposium on scattering of waves in random media and random rough surfaces.

McLaughlin, K. L., Johnson, Lane R., and McEvilly, Thomas V., (1983), Twodimensional array measurements of near-source ground accelerations, Bull. Seis. Soc. Am., 73, 349-375.

Menke, W. and Chen, R., (1984), Numerical studies of the coda falloff rate of multiply scattered waves in randomly layered media, Bull. Seis. Soc. Am., 74, 1605-1621.

Menke, W. and Dubendorff, B., (1985), Discriminating intrinsic and apparent attenuation in layered rock, Geophys. Res. Lett., 12, 721-724.

Nicoletis, L., (1981), Simulation numerique de la propagation d'ondes sismiques dans les milieux straifies a deux et trois dimensions: contribution a la construction et a l'interpretation des sismogrammes sythetiques, Ph.D. thesis, Universite Pierre et Marie Curie, Paris, France.

Ogilvy, J. A., (1987), Wave scattering from rough surfaces, Rep. Prog. Phys., 50, 15531608.

Rayleigh, L., (1878), The Theory of Sound, London, Macmillan. 
Sato, H., (1982), Amplitude attenuation of impulsive waves in random media based on travel time corrected mean wave formalism, J. Acoust. Soc. Am., 71, 559-564.

Spudich, P. and Orcutt, J., (1980a), A new look at the seismic velocity structure of ocean crust, Rev. of Geophys., and Space Phys., 18(3), 627-645.

Spudich, P. and Orcutt, J., (1980b), Petrology and porosity of an oceanic crustal site: results from wave form modeling of seismic refraction data, J. Geophys. Res., 85(B3), 1409-1433.

Stephen, R. A., (1984a), Finite difference seismograms for laterally varying marine models, Geophys. J. R. astr. Soc., 79, 184-198.

Stephen, R. A., (1984b), Synthetic vertical seismic profiles by the method of finite difference, from Toksoz M. N., and Stewart, P. 2., 1984, Vertical Seismic Profiling, Part B: Advanced concepts, Geophys. Press, , 63-79.

Stephen, R. A., (1985), Finite-difference synthetic acoustic logs, 50(10), 1588-1609.

Stephen, R. A., (1988a), Finite difference methods for bottom interaction problems, Computational Acoustics: Wave Propagation, D. Lee, R. L. Sternberg and M. H. Schultz, 225-238.

Stephen, R. A., (1988b), Lateral heterogeneity in the upper oceanic crust at DSDP Site 504, J. Geophys. Res., 93(B6),

Stephen, R. A., (1988c), A review of finite difference methods for seismo-acoustic problems at the seafloor, Rev. Geophys., 26(3), 445-458.

Stephen, R. A. and Bolmer, S. T., (1985), The direct wave root in marine seismology, Bull. Seis. Soc. Am., 75, 57-67.

Virieux, J., (1984), SH-wave propagation in heterogeneous media: Velocity-stress finitedifference method, Geophysics, 49, 1933-1957.

Virieux, J., (1986), P-SV wave propagation in heterogeneous media: Velocity-stress finite difference method, Geophysics, 51(4), 889-901.

Wu, R., (1982), Attenuation of short period seismic waves due to scattering, Geophys. Res. Lett., 9(1), 9-12.

Wu, R. S., (1983), Mean field attenuation and amplitude attenuation due to wave scattering, Wave Motion, 4, 305-316.

Wu, R. and Aki, K., (1988), Introduction: Seismic wave scattering in three-dimensionally heterogeneous earth, PAGEOPH, 128(1/2), 1-6. 


\section{CHAPTER 1}

Geoacoustic scattering from seafloor features in the ROSE area 


\title{
Geoacoustic scattering from seafloor features in the ROSE area
}

\begin{abstract}
A strong "refraction branch diffraction", presumably due to scattering from a lateral heterogeneity on or below the sea floor, has been observed on ocean bottom hydrophone data from the Rivera Ocean Seismic Experiment (ROSE). This arrival is unusual because of its coherence and relatively large amplitude. Finite difference modeling of a number of possible seafloor diffractors and associated lateral velocity variations are presented which demonstrate the occurrence and characteristics of "refraction branch diffractions". In general, the half-width of the diffractor must be approximately the same as the seismic wavelength in order to produce a strong diffraction. Velocity gradients present in the models, as well as P-S conversion, complicate the wavelength-halfwidth relationship. Three different models, a hill, a valley, and a subsurface, high-velocity block, all produced diffractions of sufficient amplitude to explain the data. There is a hill along the line with approximately the same dimensions as the model hill and it is the proposed source of the diffracted energy in the data. The large models used also clearly demonstrate the existence of phases which are theoretically possible but rarely seen in marine seismic (geoacoustic) data such as the pseudo-Rayleigh wave and the P and S interference head waves.
\end{abstract}




\section{INTRODUCTION}

Marine seismic (geoacoustic) data often contain relatively large anomalous arrivals superimposed on expected arrivals for individual or small groups of traces. Factors such as topographic focusing or crustal heterogeneities could be the cause of such anomalies. Ocean bottom hydrophone $(\mathrm{OBH})$ data from the Rivera Ocean Seismic Experiment (ROSE) contain much of this type of noise. There is one OBH line, however, which contains coherent arrivals between the refracted P-wave and the direct water wave for almost the entire ten kilometer section (figure 1.1). We call this the "refraction branch diffraction" to distinguish it from diffraction hyperbola of near normal incidence reflections. There are no simple reflection paths which can account for the travel times of the refraction branch diffraction. The arrival time behavior indicates that some type of diffraction or back-scattering is occurring at a range of 6.6 kilometers. Rough seafloor in the area, seen in figure 1.2, contains many hills and valleys, one of which is the most likely cause of this scattering. In this paper we model and discuss the phenomenon of the refraction branch diffractions.

The ability to detect heterogeneities in or on the oceanic crust is a function of the frequency of the seismic source (or the wavenumber or wavelength of the energy as it travels through the medium), the length scale of the heterogeneity and the distance travelled by the energy through the heterogeneous material. Aki and Richards ${ }^{1}$ classify scattering phenomena on the basis of the dimensionless parameters $k a$ ( $2 \pi$ times the ratio of heterogeneity length to wavelength) and $k L(2 \pi$ times the number of wavelengths travelled through the heterogeneous region). Small scale heterogeneities (small $k a$ ) can be approximated by an equivalent homogeneous bodyl and the scattering can be considered as an attenuation effect. Large scale heterogeneities (large $k a$ ) can be considered as separate 
homogeneous bodies and ray theory can be used to trace the energy propagation. However, when the size of the heterogeneity is approximately equal to the seismic wavelength, energy loss due to scattering reaches a maximum. For the problem considered here (figure 1.1) it appears that there is a single scatterer ( $k L$ small, $k a$ near 1 ) and modeling in a deterministic fashion (eg. using the method of finite differences) is appropriate.

For the purpose of travel time analysis, a scattering body can be considered as a secondary source and ray theories can be used for modeling. These methods generally assume either high frequencies or laterally homogenous media or both. Amplitude analysis and energy partitioning in media which varies laterally on the scale of seismic wavelengths are not handled well with these methods. At realistic frequencies and with laterally heterogeneous media, the wave characteristics of seismic propagation become important. Modeling schemes which preserve wave phenomena such as scattering, energy partitioning, and interference must be used under these circumstances. Synthetic modeling using the wavenumber integral or finite difference/element methods preserve wave characteristics but only the finite difference/element methods allow for lateral as well as vertical variations in structure. The finite difference method is used in this study to model the rough sea floor found in the project ROSE area. 
Figure 1.1. The refraction profile for $\mathrm{OBH} 2 \mathrm{~W}$ on airgun line 1 from the ROSE experiment (see figure 1.3 for location). The bathymetry section below the seismogram was used for the topographic travel time correction. The "refraction branch diffraction" shows as a "smile" after the primary refracted arrival (modified figure from Purdy 4 ). 


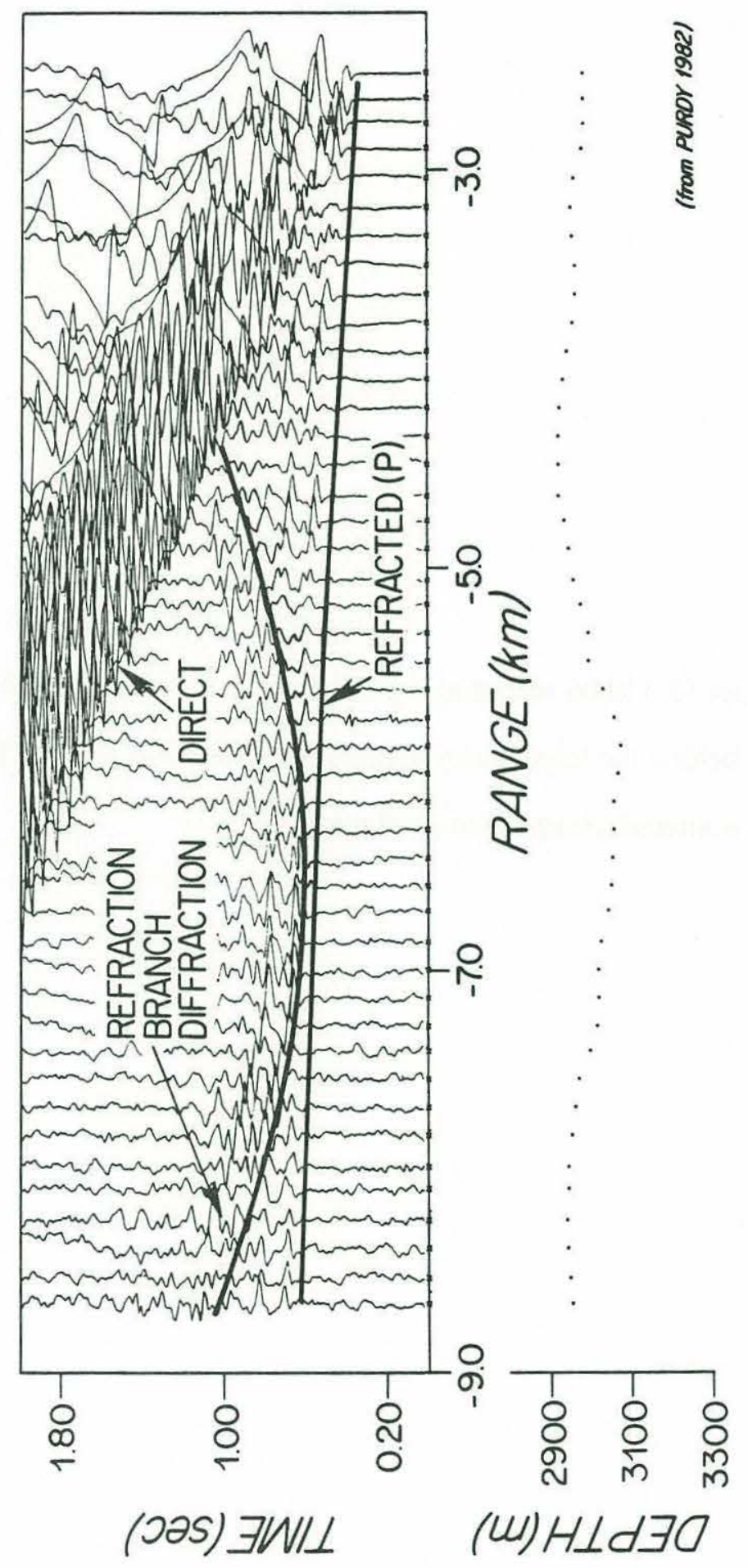


Figure 1.2. Echo sounder $(3.5 \mathrm{kHz})$ record along airgun line 1 near $\mathrm{OBH} 2 \mathrm{~W}$. Note the hill offset from the line behind the large valley centered around $7 \mathrm{~km}$ range. This hill is the proposed source of the scattered energy seen in figure 1.1. 


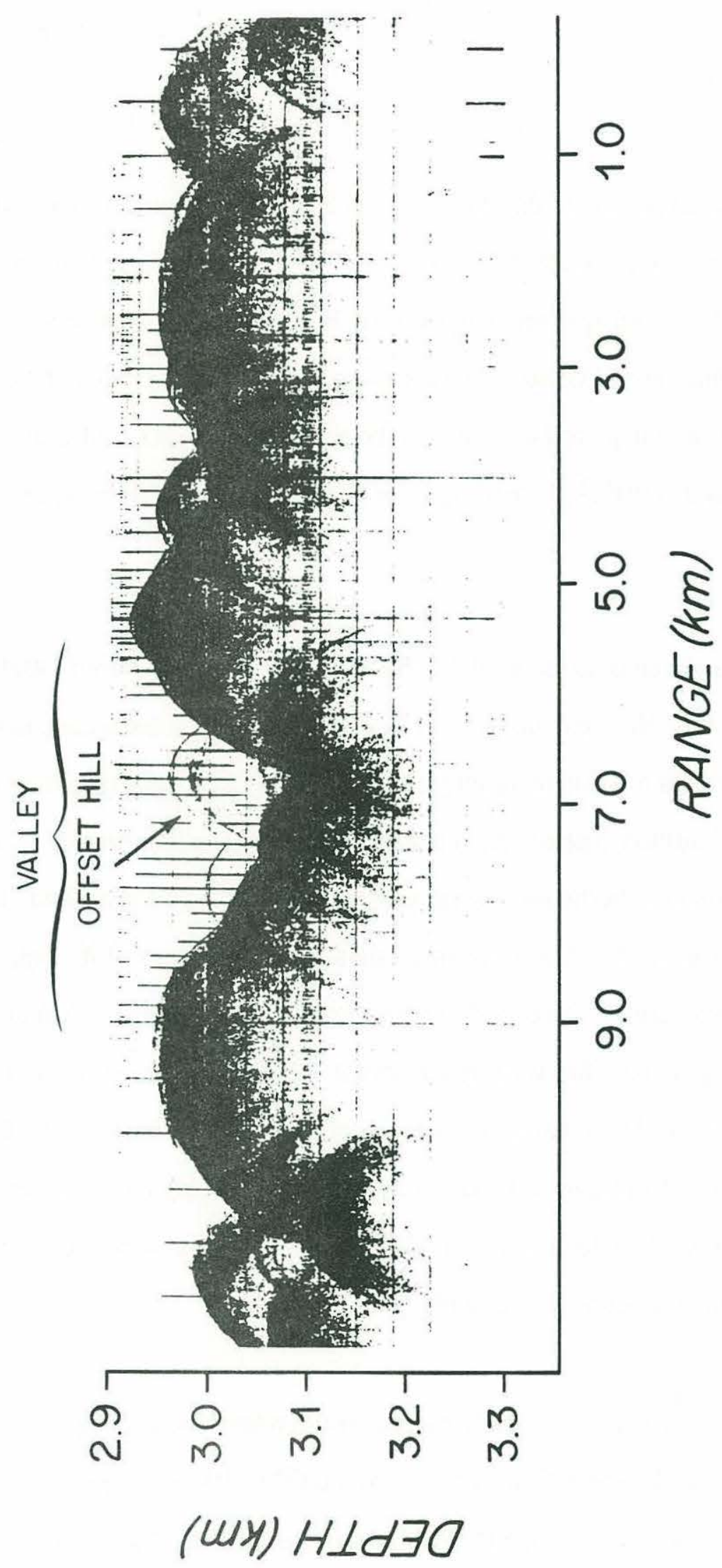




\section{DATA AREA}

The sea based portion of the Rivera Ocean Seismic Experiment took place in early 1979 and was carried out to study the evolution of young ocean lithosphere at the East Pacific Rise (EPR) spreading center (figure 1.3). Earthquake and explosive events from the EPR, the Clipperton and Orozco fracture zones, and along the coast of Mexico, were recorded for a two month period using ocean bottom seismometers (OBS) and ocean bottom hydrophones $(\mathrm{OBH})^{2}$. Crustal ages of 0-4 million years are covered by the ROSE study area.

This study is an attempt to model the diffraction seen in the data from $\mathrm{OBH} 2 \mathrm{~W}$ on airgun line 1. Four 7.604 liter (464 cu.in.) Bolt $1500 \mathrm{C}$ air guns were used to shoot the line, with five OBH's for data recording (figure 1.3). The crustal age below $\mathrm{OBH} 2 \mathrm{~W}$ is approximately 0.5 million years ${ }^{3}$. Data from this line were used primarily by Purdy ${ }^{4}$ to determine the variability in the seismic structure of oceanic crustal layer two. Ewing and Purdy 5 also used part of this data to constrain the velocity structure of the upper 500-800 meters of the oceanic crust. Velocity-depth profiles used to model the crust around $\mathrm{OBH}$ $2 \mathrm{~W}$ are given in figure 1.4. Separate upper crustal velocity structures of Purdy 4 and Ewing and Purdy 5 are shown combined with a lower crustal P-velocity of $6.0 \mathrm{~km} / \mathrm{sec}$. Swave velocities shown in figure 1.4 were inferred by considering the upper crust as a Poisson solid (Poisson's ratio $(s)=0.25$ ). The upper velocity structure of Purdy 4 was used for most of the modeling in this study.

Figure 1.2 shows a portion of a bathymetric profile taken along airgun line 1 in the vicinity of $\mathrm{OBH} 2 \mathrm{~W}$. In general, the terrane around $\mathrm{OBH} 2 \mathrm{~W}$ is rough. The striking feature of the bathymetry around $\mathrm{OBH} 2 \mathrm{~W}$ is the presence of a relatively large valley with 


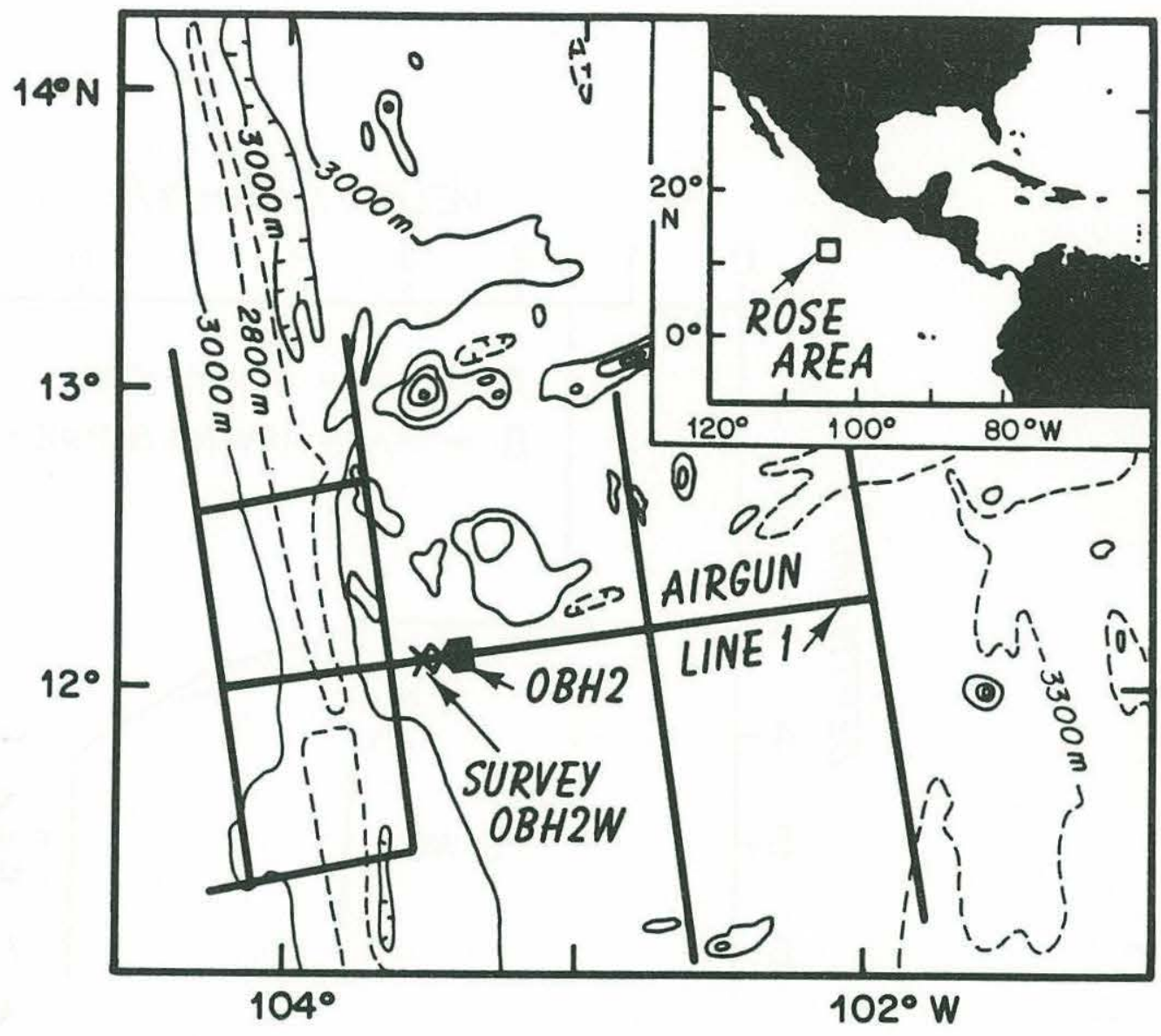

Figure 1.3. Location of $\mathrm{OBH} 2$ along airgun line 1 of the ROSE experiment. Refraction data seen in figure 1 (survey OBH $2 \mathrm{~W}$ ) includes shots ranging from 0 to $9 \mathrm{~km}$ west of OBH 2. Inset shows general location of the ROSE study area (modified figure from Ewing and $\operatorname{Meyer}^{2}$ ). 


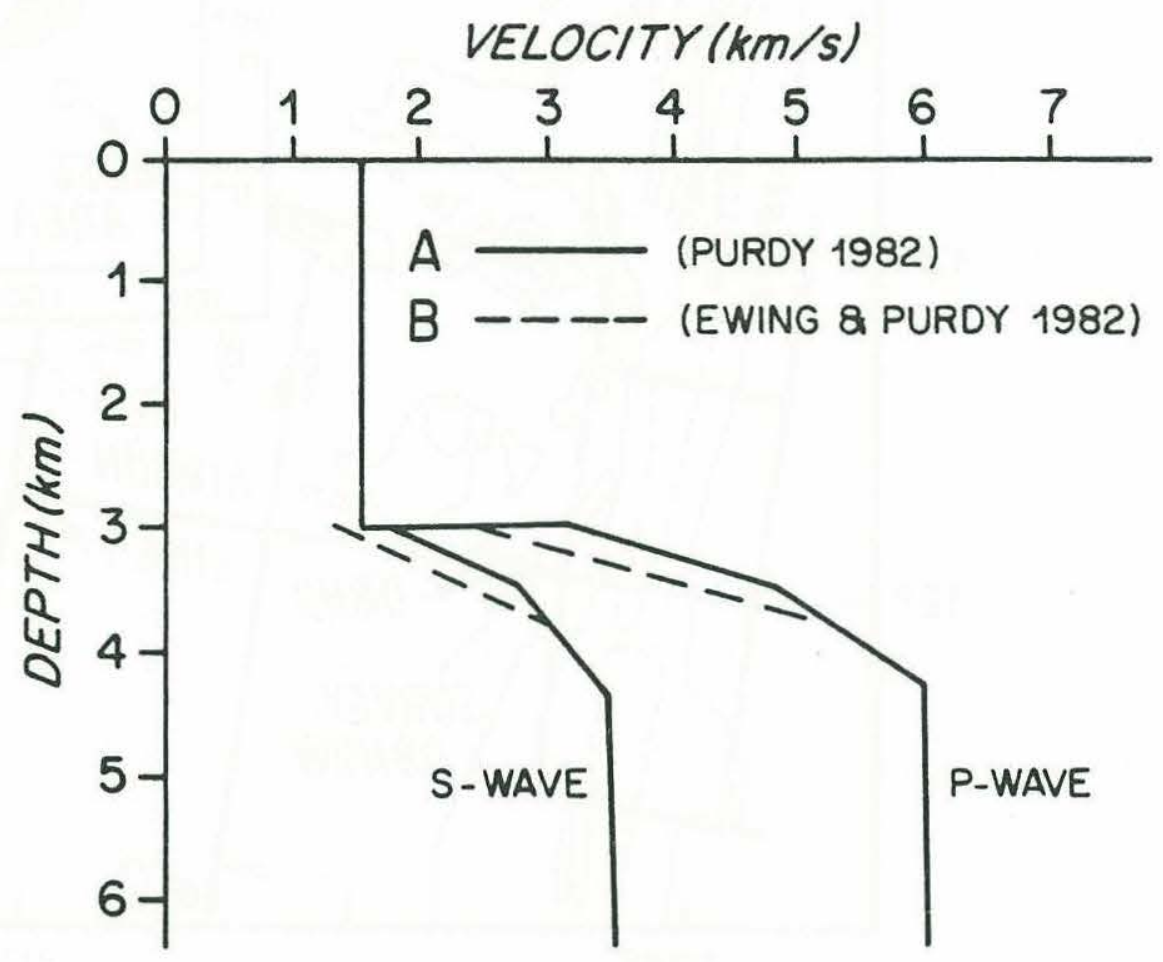

Figure 1.4. Velocity-depth profiles used for finite difference models. Profile 'A' was used for FLAT, HILL, VALLEY1, VALLEY2, and BLOCK models, and profile 'B' was used for VALLEY3. 
a hill offset behind it. Large valleys such as this one are generally not seen along the rest of the line. While there are many hill structures along the line, little can be said about their three dimensional extent, and no initial conclusions can be drawn from the bathymetry alone. It is possible, for example, that the dark hills in figure 1.2 are, in fact, lineated structures and the hill offset behind the valley is circular. There is some 3-D seabeam data along the crest of the EPR, but the data stops just to the west of the area of OBH $2 \mathrm{~W}$ (Macdonald, 1985, personal communication).

\section{TRAVEL TIME ANALYSIS}

The seismogram from $\mathrm{OBH} 2 \mathrm{~W}$ (figure 1.1) is corrected for local ocean bottom

topography using the method of Purdy6. The diffracted arrival appears symmetric about a range of approximately 6.6 kilometers. The minimum difference in travel time (DT) between the refracted and diffracted arrivals is about 0.13 seconds (at $6.6 \mathrm{~km}$ ) and the maximum DT for the ranges available is around 0.53 seconds (at 4.6 and $8.6 \mathrm{~km}$ ). These DT's are subjective and depend on the correct picks for the incidence of the diffraction. It is clear, however, that the shape of the diffraction is fairly well determined and that only its position in time will change with different picks. The general shape of the diffracted arrival, as well as the DT's were modeled first with simple ray theory. Also, the effects of moving the diffracting body along and out of the sagittal plane of the model were studied. These analyses follow for both sea floor and subsurface diffractors.

For the purpose of travel time analysis, the sea floor hill and the buried body were treated as point diffractors which act as secondary sources of seismic energy. That is, when plane wave energy reaches the point diffractor, it is excited and radiates energy in all directions. This is opposed to refraction through the body which would send out energy in only one direction, or for a sharp, flat interface, in a finite number of directions, from 
any one incoming plane wave front. Stephen 7 used the finite difference method to study refractions through a sea floor hill. Although the geometry of Stephen's model is different than that used for this study, it is obvious that there would be no back-scattering of energy from the hill by considering only refraction phenomena. Rather, diffraction from a sea floor hill is necessary to explain the back-scattered energy. Size and shape of the hill were not considered for travel time analysis.

Figure 1.5 shows the parameters and ray diagram for the sea floor hill model which is simply a layer over a half-space. For each super-critical range there are three simple paths for compressional energy to reach the $\mathrm{OBH}$; directly through the water, refracted at the critical angle, and diffracted from the hill. These account for the first three arrivals in the data. With the hill acting as a secondary source, simple ray tracing and Snell's law were used to create the travel time plots of figure 1.6. Diffraction curves for the hill at various ranges in the sagittal plane and at various offsets out of the sagittal plane are shown. The DT's are very similar to those from the data (figure 1.1). The diffracted and refracted phases arrive closest in time at a range of approximately $7.2 \mathrm{~km}$ for a hill at $6 \mathrm{~km}$. in figure 1.6a. At points two kilometers away on either side of $7.2 \mathrm{~km}$. (5.2 and $9.2 \mathrm{~km}$.), the offset between the two arrivals has increased by approximately 0.35 seconds (DT). The corresponding DT in the data is about 0.40 seconds.

Since the position of the hill does not affect the direct or refracted arrivals (for this simple analysis) the traces for a number of different hill locations within the sagittal plane are shown superimposed in one plot in figure 1.6a. As expected, the shapes of the refraction branch diffractions are the same for the different hill locations. Moving the hill away from the sagittal plane (into or out of the page in figure 1.5) effects mainly the vertical placement of the diffraction (in time) with only slight changes in its shape (figure 1.6b). It should be noted that the model geometry used in these simple models, as well as 
in the buried body model below, is the same as the data geometry. For the finite difference modeling, the two geometries are not the same and care must be taken not to confuse them 7 .

Ray tracing for the buried body model was done using velocity-depth profile A of figure 1.4. Again, as with the sea floor hill model, horizontal movement of the diffractor at a given depth within the sagittal plane does not alter the shape of the diffraction, only the horizontal placement of it with respect to the first refracted arrival (figure 1.7a). However, the shape of the diffraction can be changed by moving the diffractor vertically in the upper crust. Moving the diffractor deeper tends to flatten out the diffracted arrival shape, as seen in figure $1.7 \mathrm{~b}$. Vertical movement of the buried body within the upper crust also moves the diffracted arrival vertically in time. If the body is much below the sea floor (more than about 300 meters), the diffraction arrives before the sea floor refraction at large ranges. Of course, by moving the body away from the sagittal plane, the diffracted arrival may also be moved vertically. Because of this, the possible depth range for the diffractor is not well constrained. However, by choosing the right combination of diffractor depth and lateral offset, the DT's for this model can easily be made to match those seen in the data.

Three important facts have been brought out by ray tracing. First, and most important, is that assuming diffractions will occur from a hill or buried body, the DT's seen in the data can be reproduced by the simple models shown above. Minor changes in diffracted arrival shape, from depth difference or different velocity-depth profiles, do not greatly affect the DT's. Second, the shape of the diffraction is not significantly affected by changes in horizontal position(up to $2 \mathrm{~km}$ ) of the diffracting body either parallel or perpendicular to the sagittal plane. Because of this, the horizontal placement of the diffractor in the finite difference models does not have to be exactly the same as that which 
Figure 1.5. Geometry used for ray tracing models (also the same as data geometry).

Three ray paths from each shot to the $\mathrm{OBH}$ are responsible for the refracted, diffracted and direct arrivals seen in Figure 1.1. 


\section{RANGE $(\mathrm{km})$}

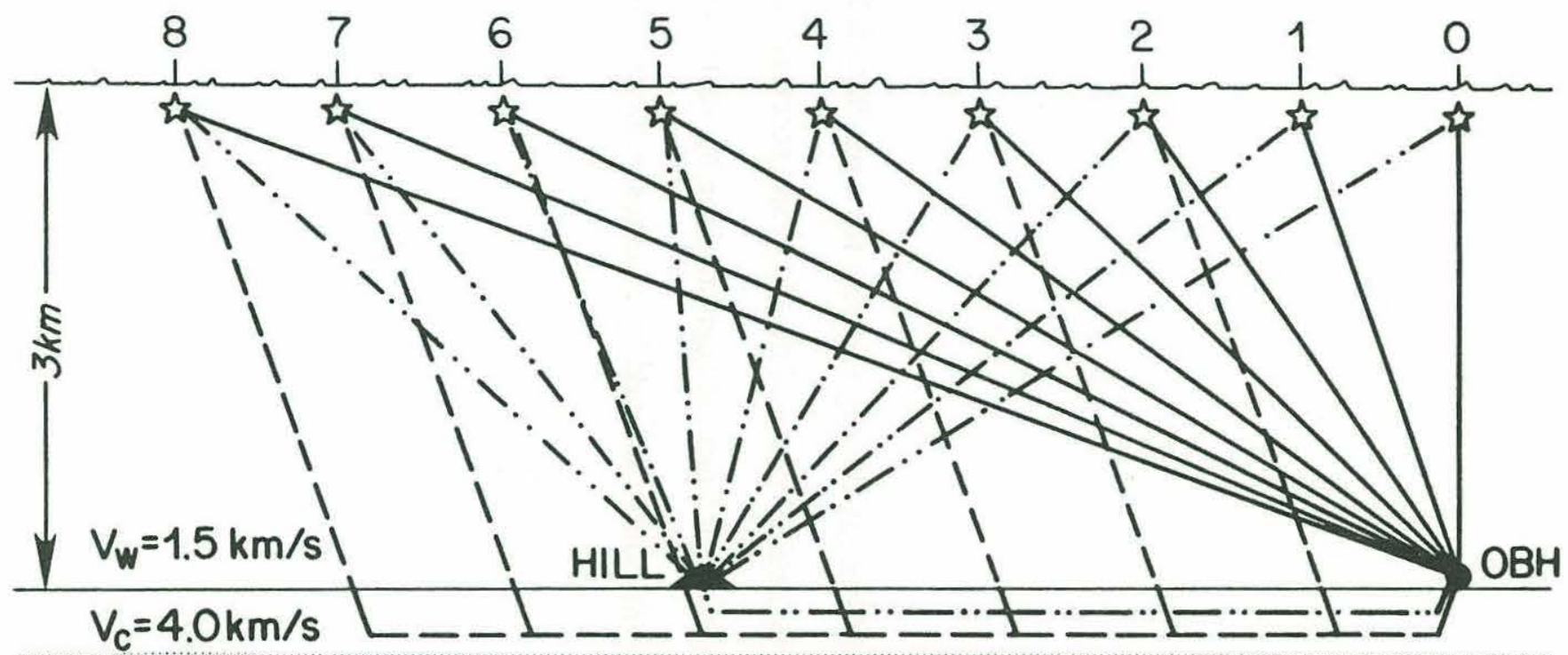

$$
\begin{gathered}
\text { DIRECT } \\
\hline--- \text { REFRACTED } \\
-\cdots-\cdot-\text { DIFFRACTED } \\
\text { ↔ } \quad \text { SHOT }
\end{gathered}
$$


Figure 1.6. Travel time plots created from ray tracing analysis for a sea floor hill. Figure a) shows hills at different ranges in the sagittal plane of the shots and receiver. Figure b) shows hills at a fixed range $(6 \mathrm{~km})$ and offset from the sagittal plane by different distances. The shape of the diffraction is not significantly affected by changes in the position of the hill (up to $2 \mathrm{~km}$ ) in either case. 


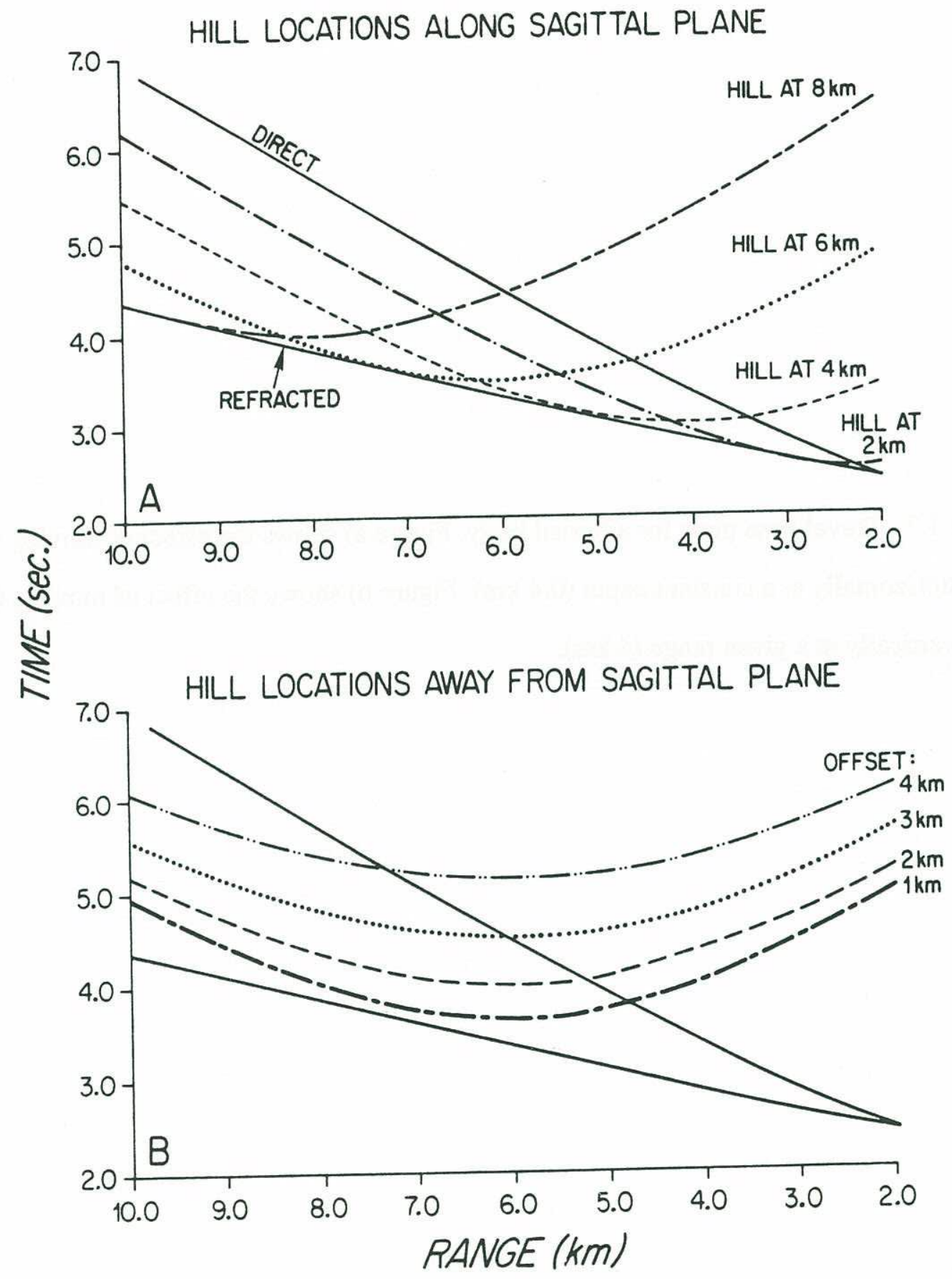


Figure 1.7. Travel time plots for a buried body. Figure a) shows the effect of moving the body horizontally at a constant depth $(0.4 \mathrm{~km})$. Figure b) shows the effect of moving the body vertically at a given range $(4 \mathrm{~km})$. 


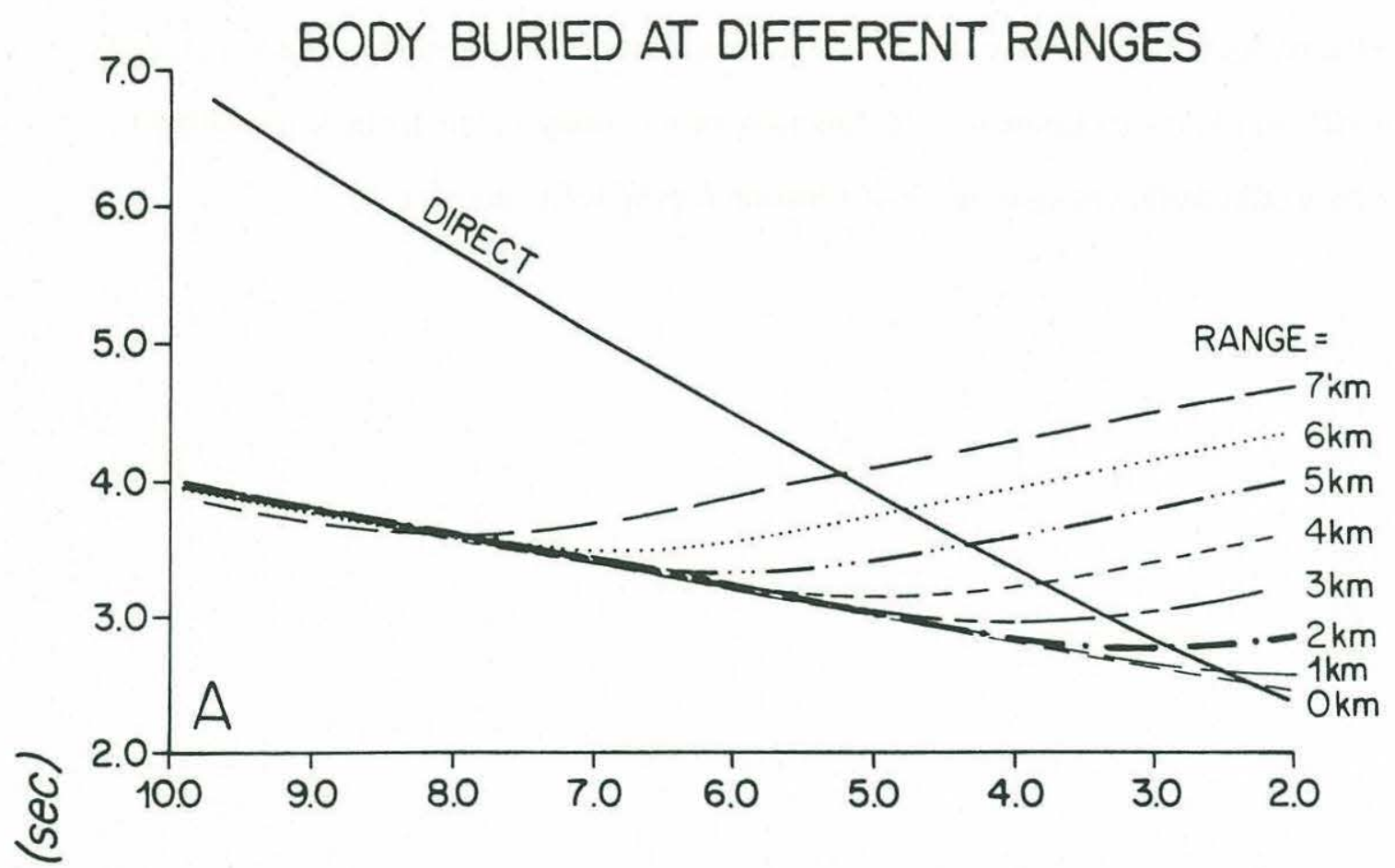

BURIED BODY AT DIFFERENT DEPTHS

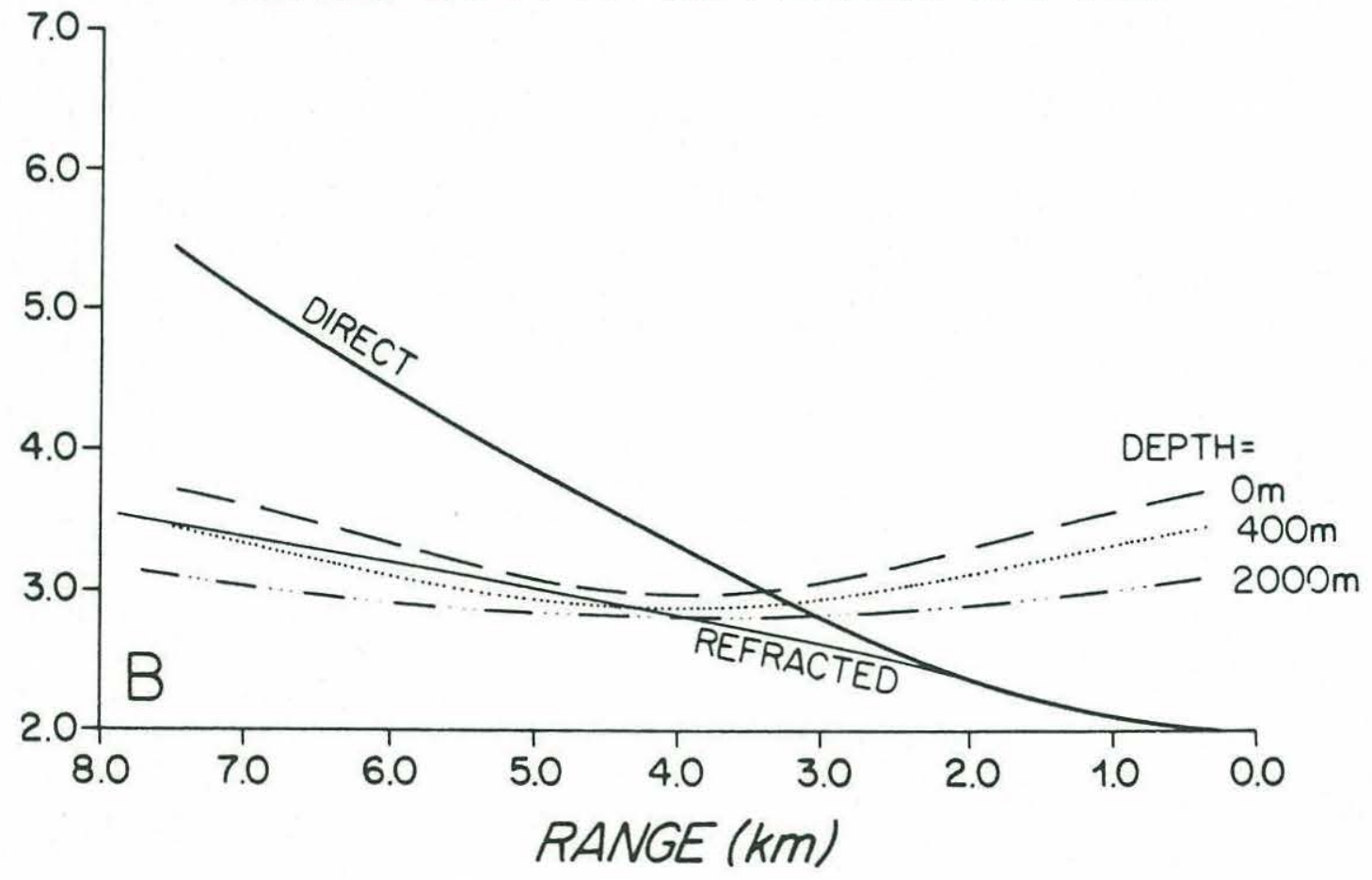


produced the data. Finally, the shape of the diffraction can be changed by altering the velocity-depth function (the shape changes between the hill model and the buried body model because of a change in $\mathrm{V}-\mathrm{Z}$ function) or by changing the depth of the diffractor (which effectively changes the V-Z function for the scattered energy). 


\section{FINITE DIFFERENCE MODELING}

Amplitude and phase conversion, as well as travel time, of the seismic energy need to be reconstructed in order to get a complete solution of the problem. This section discusses synthetic seismograms and wavefront 'snapshots' generated by a finite difference solution of the elastic wave equation for heterogenous media;

$$
\rho \ddot{\mathbf{u}}=(\lambda+\mu) \nabla(\nabla \bullet \mathbf{u})+\mu \nabla^{2} \mathbf{u}+\nabla \lambda(\nabla \bullet \mathbf{u})+\nabla \mu \times(\nabla \times \mu)+2(\nabla \mu \bullet \nabla) \mathbf{u}
$$

where $\mathbf{u}$ is the displacement vector, $\rho$ is density, $\lambda, \mu$ are Lamé's elastic parameters, $\nabla$ is the del vector operator and double dots signify the second time derivative. Some finite difference schemes 8,9 are unstable at liquid-solid boundaries and require specifically coded boundary conditions. A differencing scheme developed by Nicoletis ${ }^{10}$ and Bhasavanijall is used for the solutions in this study. In this scheme, the elastic (Lamé's) parameters and density at a point are calculated from defined velocities at eight surrounding points. In this way, a smoothing of parameters takes place at boundaries and the scheme is stable. Specific boundary conditions for the liquid-solid interface are therefore not needed with this formulation.

Accuracy and stability of the solution depend on the actual differencing scheme used and on the time and/or space increments. Stephen ${ }^{8}$ compared his finite difference solution to the reflectivity method of Fuchs and Müller 12 and found that when boundary conditions of the second order were used there is excellent agreement between the two methods. Bhasavanijall compared his results to Fourier synthesis waveforms and also found 
excellent agreement. If the space increments are too large, the seismic energy will appear to disperse, with low frequencies travelling faster than high frequencies ${ }^{13}$. Kelly et. al. ${ }^{13}$ found that dispersion is minimal if there are at least ten grid points per minimum seismic wavelength. Instabilities caused by insufficient time sampling can be avoided if the time increment satisfies;

$$
\Delta t=h /\left(\alpha^{2}+\beta^{2}\right) 1 / 2 \quad, \quad \alpha=((\lambda+2 \mu) / \rho)^{1 / 2}, \beta=(\mu / \rho)^{1 / 2}
$$

where $h$ equals the space increment (horizontal and vertical increments are equal for these models), $\alpha$ is the P-wave velocity, and $\beta$ is the $S$-wave velocity.

Figure 1.8 shows the set up of the general finite difference grid. Only the points in the transition zone are calculated using the heterogenous finite difference scheme of Bhasavanija. The layers above and below the transition zone are homogeneous (both vertically and horizontally) and are solved using a homogeneous solution. The source is introduced along the top of the grid and, for potential, is in the form of the first derivative of a Gaussian curve ${ }^{13}$. Absorbing boundaries ${ }^{14-16}$ are used at maximum range and depth and there is an axis of symmetry at zero range.

As stated earlier, the geometry of the finite difference models is different than the geometry of the data and ray tracing models. For the data, there is one $\mathrm{OBH}$ and a line of shots at the sea surface (figure 1.5). The situation is opposite in the finite difference models with one surface shot and a line of receivers at the sea floor (figure 1.9a) (although the vertical position of the receivers can be varied). Travel time plots for the two setups are quite different for a hill at the same range (compare figures 1.6 and $1.9 \mathrm{~b}$ ). Only one trace is common to both the data and any given model geometry, depending on the shot- 
diffractor separation. To actually synthetically reproduce the data, one model geometry must be run for every shot-receiver arrangement in the data. By changing the location of the diffractor in the model, a different shot-receiver range of the data can be reproduced.

\section{A Flat Model}

A control model with a flat sea floor and velocity-depth profile A of figure 1.4 was first run to produce the expected arrivals for an area without diffractors. The different phases and partitioning of energy can be seen in wavefront 'snapshots' (figure 1.10) of divergence and curl of displacement. Compressional and shear energy are proportional to the squares of divergence and curl of displacement respectively, and the snapshots can be considered as representations of compressional and shear wave energy travelling across the grid. The curl amplitudes have been scaled by (3) $1 / 2$ times the divergence scaling in order to best represent the relationship to energy. Snapshots were output every 0.4 seconds ( 320 time steps) with the first frame at two seconds.

All of the phases expected for the FLAT model can be seen in figure 1.10. The nomenclature given in brackets is after Brekhovskikh 17 and is appropriate for the phases present in the case of a sharp interface between two media of constant velocities. These symbols are not exactly correct for the phases seen in the models for this study because of the presence of velocity gradients below the seafloor. If the gradients were allowed to decrease to zero, the phases seen in the snapshots would degenerate to those given in brackets. Brekhovskikh's nomenclature is used here because it closely approximates the system of waves which exist in media with velocity gradients below a sharp interface.

The source is located $2 \mathrm{~km}$ above the top of the grid and the direct water wave [P1] is just above the seafloor in the 2.0 second snapshot (see figure 1.10 ). At 2.4 seconds the 
Figure 1.8. Setup of the grid for finite difference models. Range and depth increments are equal to 10 meters (figure not to scale). Each model was run for 4000 time steps of 0.00125 seconds each. The source is introduced along the top boundary of the grid. The bottom and right hand side boundaries are absorbing. The left hand boundary is an axis of symmetry. 


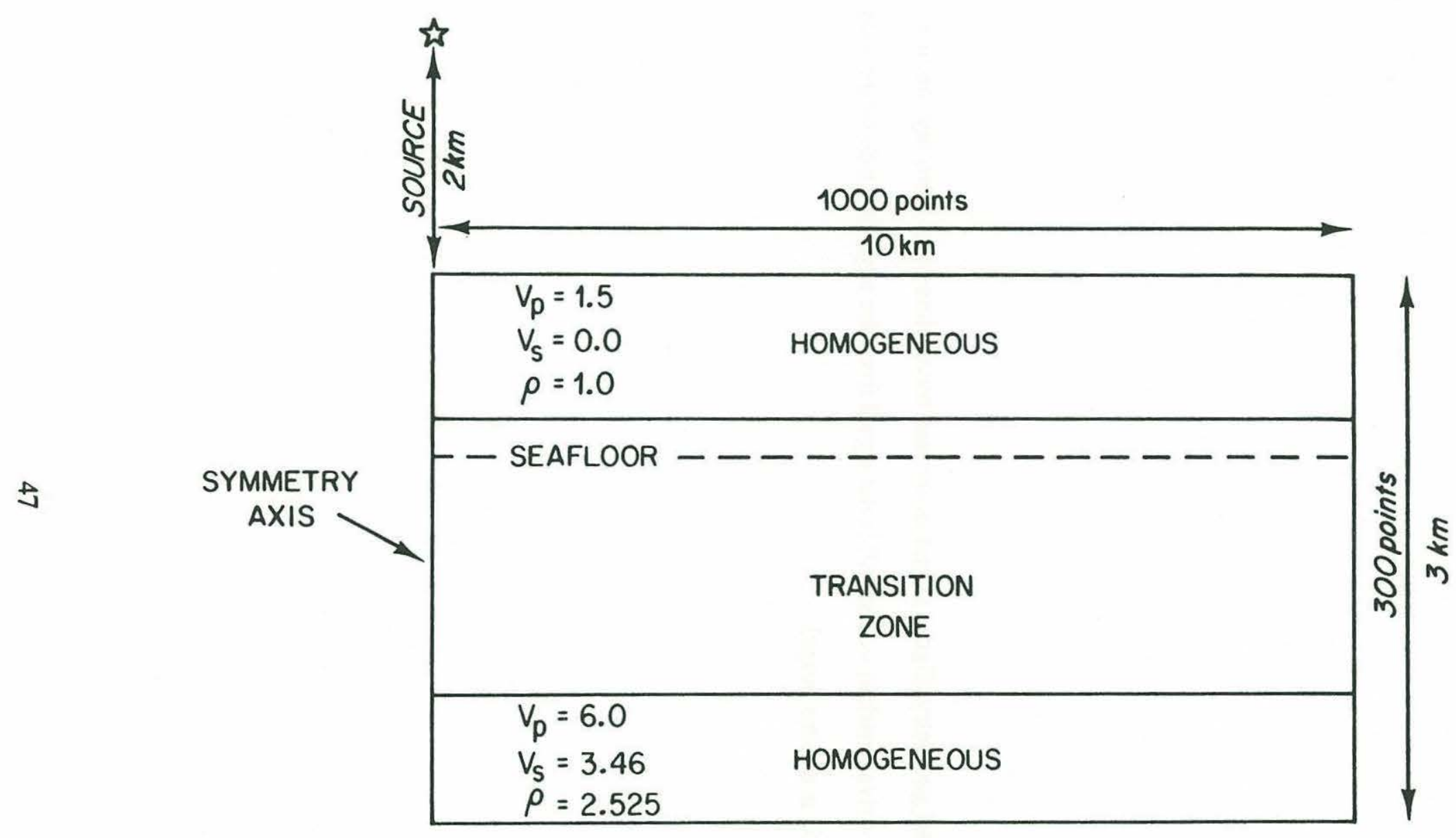


Figure 1.9. Model geometry (figure a) and associated travel time plots from ray tracing (figure b). The arrival marked virtual hill is the signal from the opposite side of the axis of symmetry (axis is at zero range). 

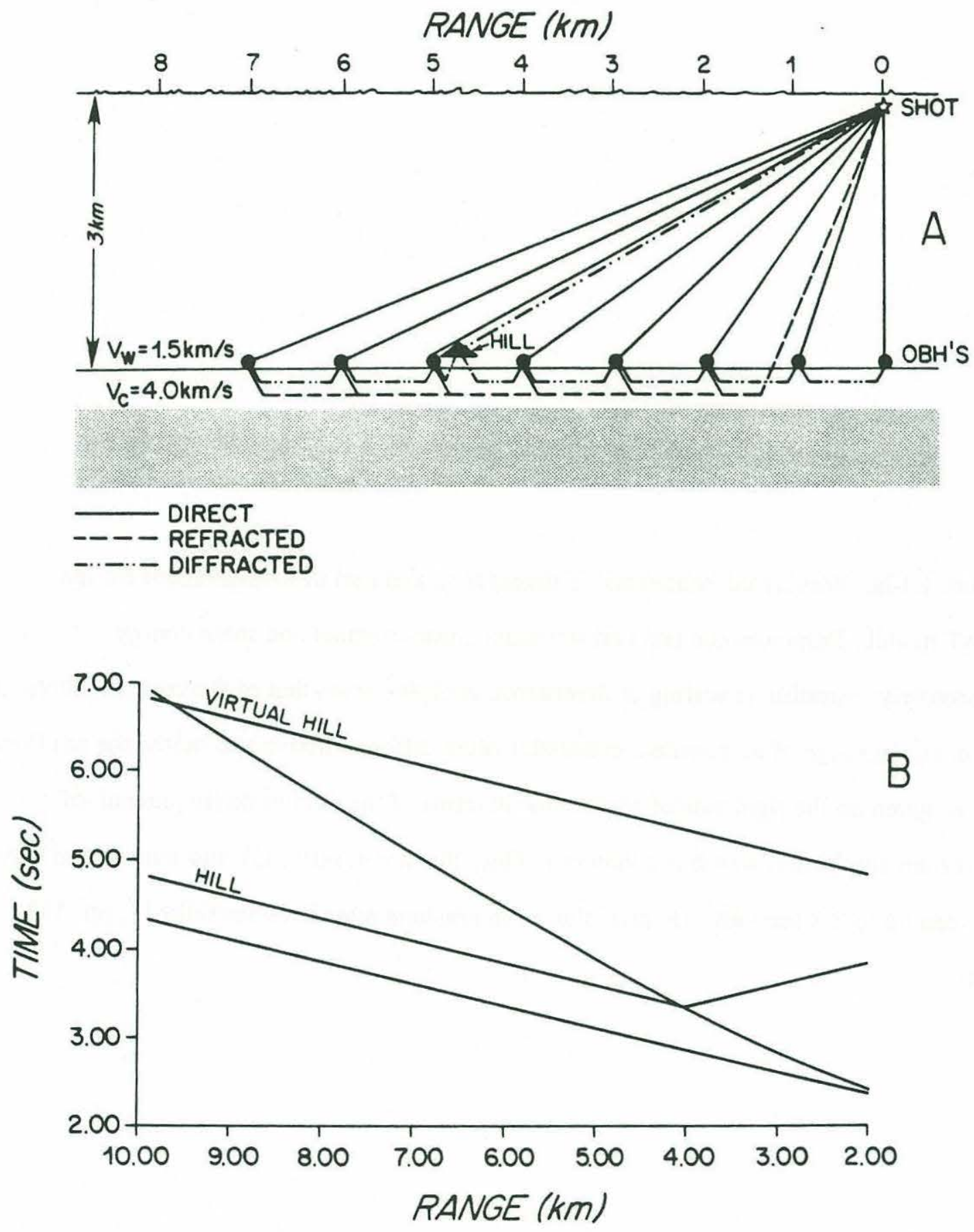
Figure 1.10a. Wavefront 'snapshots' of divergence and curl of displacement for the FLAT model. Displacement and curl represent compressional and shear energy respectively. Amplitude scaling of divergence is (3) ${ }^{1 / 2}$ times that of the curl snapshots. In addition, the range of amplitude contoured is often different above and below the sea floor and is given on the right side of each frame in terms of the amplitude (in percent of maximum amplitude) which is contoured. Only the direct, reflected, and transmitted waves are seen up to 2.8 seconds. (Nomenclature in brackets after Brekhovskikh ${ }^{17}$, pp. 318324). 


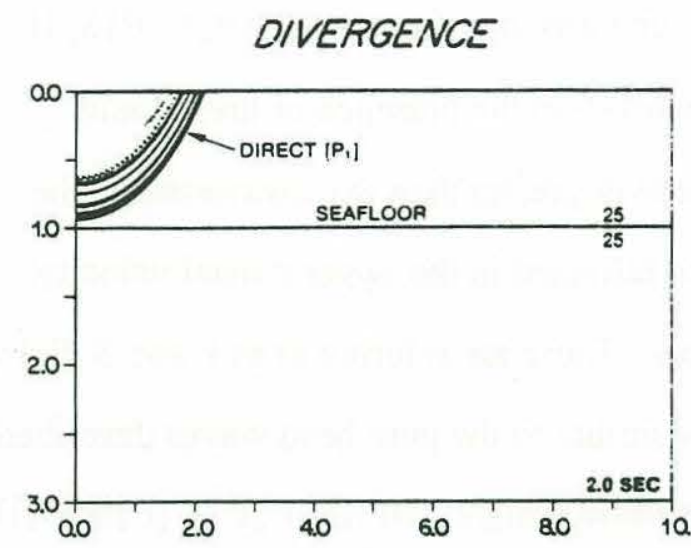

FLAT MODEL

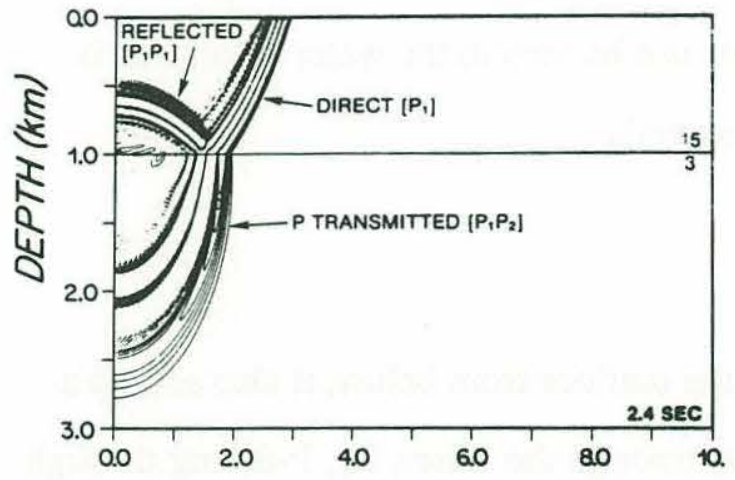

CURL
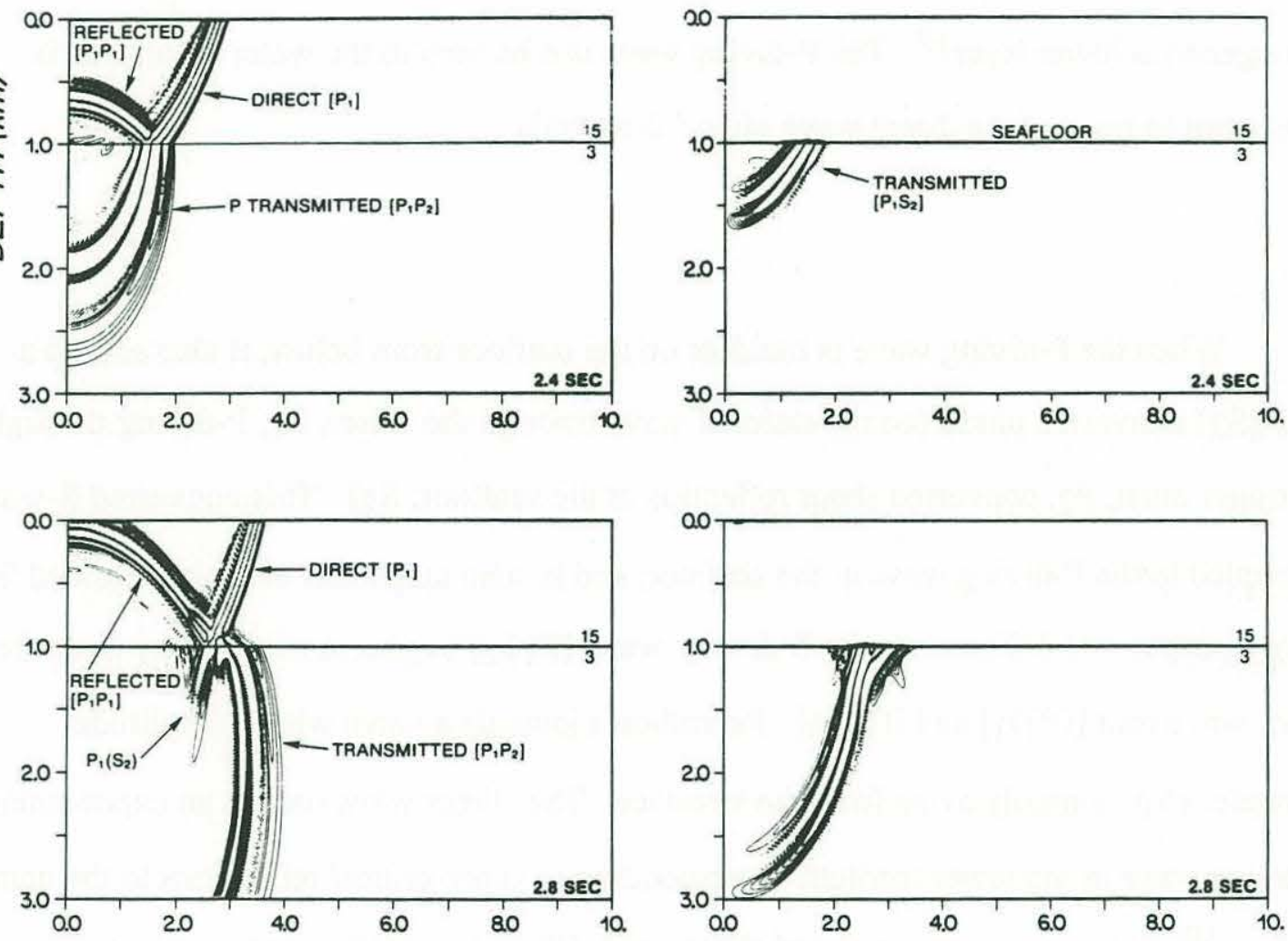

RANGE $(\mathrm{km})$ 
direct wave has reached the seafloor and partitioned into direct $\left[\mathrm{P}_{1}\right]$, transmitted $\left[\mathrm{P}_{1} \mathrm{P}_{2}\right]$, and reflected $\left[\mathrm{P}_{1} \mathrm{P}_{1}\right]$ compressional waves and a transmitted converted shear wave $\left[\mathrm{P}_{1} \mathrm{~S}_{2}\right]$. If the lower medium were a uniform half-space the transmitted waves $\left(\left[\mathrm{P}_{1} \mathrm{P}_{2}\right],\left[\mathrm{P}_{1} \mathrm{~S}_{2}\right]\right)$ would propagate out the bottom of the grid. However, in the presence of the velocity gradients, portions of these waves with ray parameter greater than the slownesses at the bottom of the grid ( 0.17 for $\mathrm{P}$ and 0.29 for $\mathrm{S}$ ) are refracted in the upper crustal velocity gradients and impinge on the seafloor from below. These are referred to as $\mathrm{P}$ and $\mathrm{S}$ diving waves. In the upper layer they are kinematically similar to the pure head waves described in Brekhovskikh 17 and we have given them the same designation ( $\left.\left[\mathrm{P}_{1} \mathrm{P}_{2} \mathrm{P}_{1}\right],\left[\mathrm{P}_{1} \mathrm{~S}_{2} \mathrm{P}_{1}\right]\right)$. The pure head waves do not exist in our model because they are only defined for a homogeneous lower layer ${ }^{18}$. The P-diving wave can be seen in the water column as it moves out in front of the direct wave after 3.2 seconds.

When the P-diving wave is incident on the seafloor from below, it also sets up a $\left[\mathrm{P}_{1} \mathrm{P}_{2} \mathrm{~S}_{2}\right]$ converted phase (compressional wave through the water, $\mathrm{P}_{1}, \mathrm{P}$-diving through the upper crust, $\mathrm{P}_{2}$, converted shear reflection at the seafloor, $\mathrm{S}_{2}$ ). This converted $\mathrm{S}$-wave is coupled to the P-diving wave at the seafloor, and is also tangential to the transmitted Swave at depth. At 3.6 seconds the $\mathrm{S}$-diving wave $\left[\mathrm{P}_{1} \mathrm{~S}_{2}\right]$ begins to move away from the direct wave root $\left[\left(\mathrm{P}_{1}\right)_{1}\right]$ and $\left[\left(\mathrm{P}_{1}\right)_{3}\right]$. Parentheses indicate a wave whose amplitude decreases exponentially away from the interface. The 'direct wave root' is an exponentially decaying wave in the lower medium corresponding to super-critical reflections in the upper medium ${ }^{19}$. It has compressional and shear parts, $\left[\left(\mathrm{P}_{1}\right)_{1}\right]$ and $\left[\left(\mathrm{P}_{1}\right)_{3}\right]$ respectively. Coupled to the S-diving wave $\left[\mathrm{P}_{1} \mathrm{~S}_{2}\right]$ is a $\mathrm{P}$-wave whose amplitude decreases exponentially with depth $\left[\mathrm{P}_{1}\left(\mathrm{~S}_{2}\right)\right]$. 
Interference waves ${ }^{18}$, which are essentially the superposition of diving wave multiples incident on the seafloor from below, occur in the snapshots at 3.2 and 4.0 seconds respectively. After they have separated from the principle diving waves, these waves appear very much like pure head waves in the water column and are referred to as interference head waves ${ }^{18}$. The interference P-head wave can be seen at 3.6 seconds but the interference S-head wave cannot be seen since it does not develop as a distinct phase until after the last snapshot (later than 4.4 seconds). The only other major phase to occur is the pseudo-Rayleigh wave which appears in the 4.4 second snapshot. This phase is an evanescent wave in the crust, with both divergence and curl componenets, and it appears as a plane propagating wave in the water.

Along with these expected arrivals, there are a few phases in the snapshots which are artifacts of the grid and the numerical method. The P-diving wave reflects from the top of the grid (seen in the 4.0 second snapshot of figure 1.10) because this boundary is rigid after the source is introduced. Also, there is some noise in the later snapshots along the bottom of the grid due to incomplete absorption of energy along this boundary. The phases in the water column of the curl snapshots are artifacts of reflection from the upper rigid boundary. Water column wavefronts which occur in the snapshots of the curl of displacement (figure 1.10) would not be present if shear energy was plotted. Since the shear velocity in water is zero, the amplitude of the shear energy in the water would also be identically zero. P-S conversion below the water-solid interface is accurate and complete. None of these problems interfere with any phases of interest for this study.

Figure 1.11 shows the time series of pressure for receivers at depths of 2.48 and 2.98 $\mathrm{km}$. and of vertical displacement for receivers at $3.48 \mathrm{~km}$ depth. The time series in figures 1.1la and $1.1 \mathrm{lb}$ are very similar except for slight offsets of the arrivals in time and space (between the two seismograms) and the number of water wave multiples. The seafloor 
Figure 1.10b. Continuation of the Wavefront 'snapshots' of divergence and curl of displacement for the FLAT model seen in figure 1.10a. Note that the range window moves with the wavefronts. At 3.2 seconds the P-diving wave can be seen as a plane wave in the water and the direct wave root 19 travels below the sea floor directly beneath the direct wave. (Nomenclature in brackets after Brekhovskikh17, pp. 318-324). 


\section{FLAT MODEL}
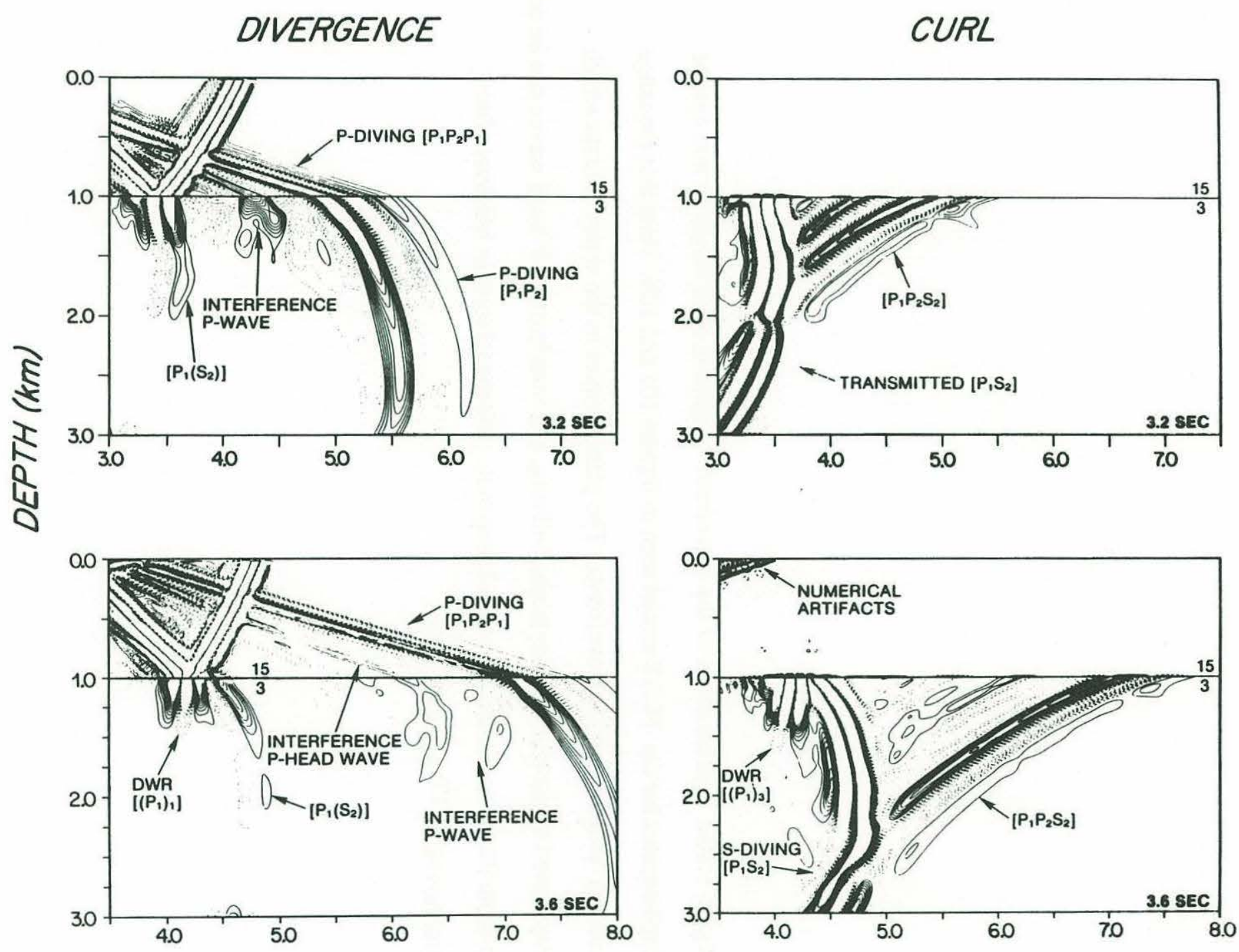

RANGE (km) 
Figure 1.10c. Continuation of the wavefront 'snapshots' of divergence and curl of displacement for the FLAT model seen in figures 10a and 10b. Note that the range window moves with the wavefronts. The planar waves in the water column which correspond to the pseudo- Rayleigh, S-diving, and interference P-head waves can be seen as separate phases in the 4.4 second snapshot. (Nomenclature in brackets after Brekhovskikh ${ }^{17}$, pp. 318-324). 


\section{FLAT MODEL}

\section{DIVERGENCE}

y

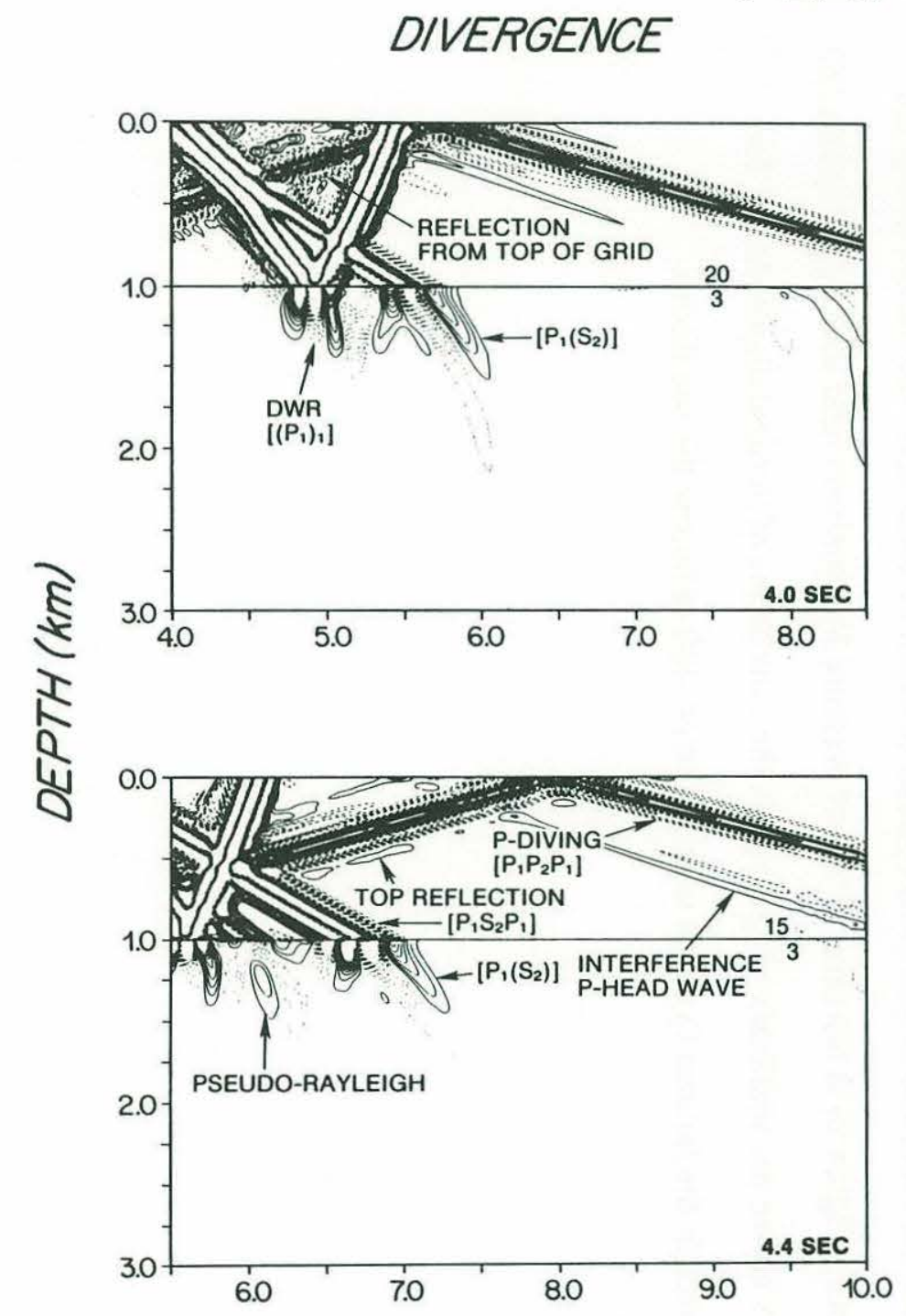

CURL
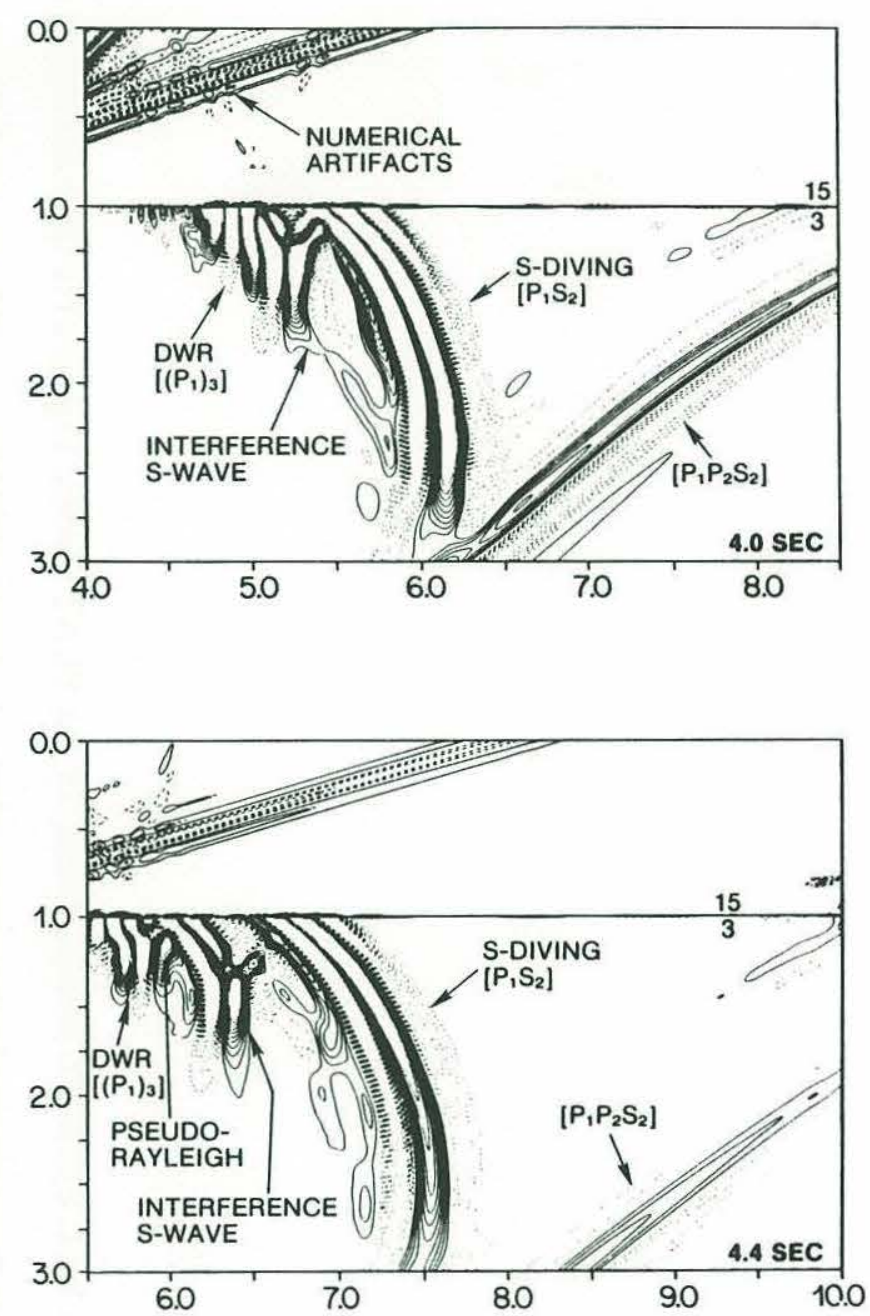

RANGE $(\mathrm{km})$ 
Figure 1.11. Synthetic seismograms for the FLAT model. Figure a) is the time series of pressure for receivers in the water column (a depth of $2480 \mathrm{~m}$ or 520 meters above the seafloor). Figure b) is the time series of pressure for receivers near the ocean bottom (20 meters above the seafloor). Figure $\mathrm{c}$ ) is the time series of vertical displacement for receivers in the bottom (a depth of $3480 \mathrm{~m}$ or $480 \mathrm{~m}$ below the sea floor). 


\section{FLAT MODEL}
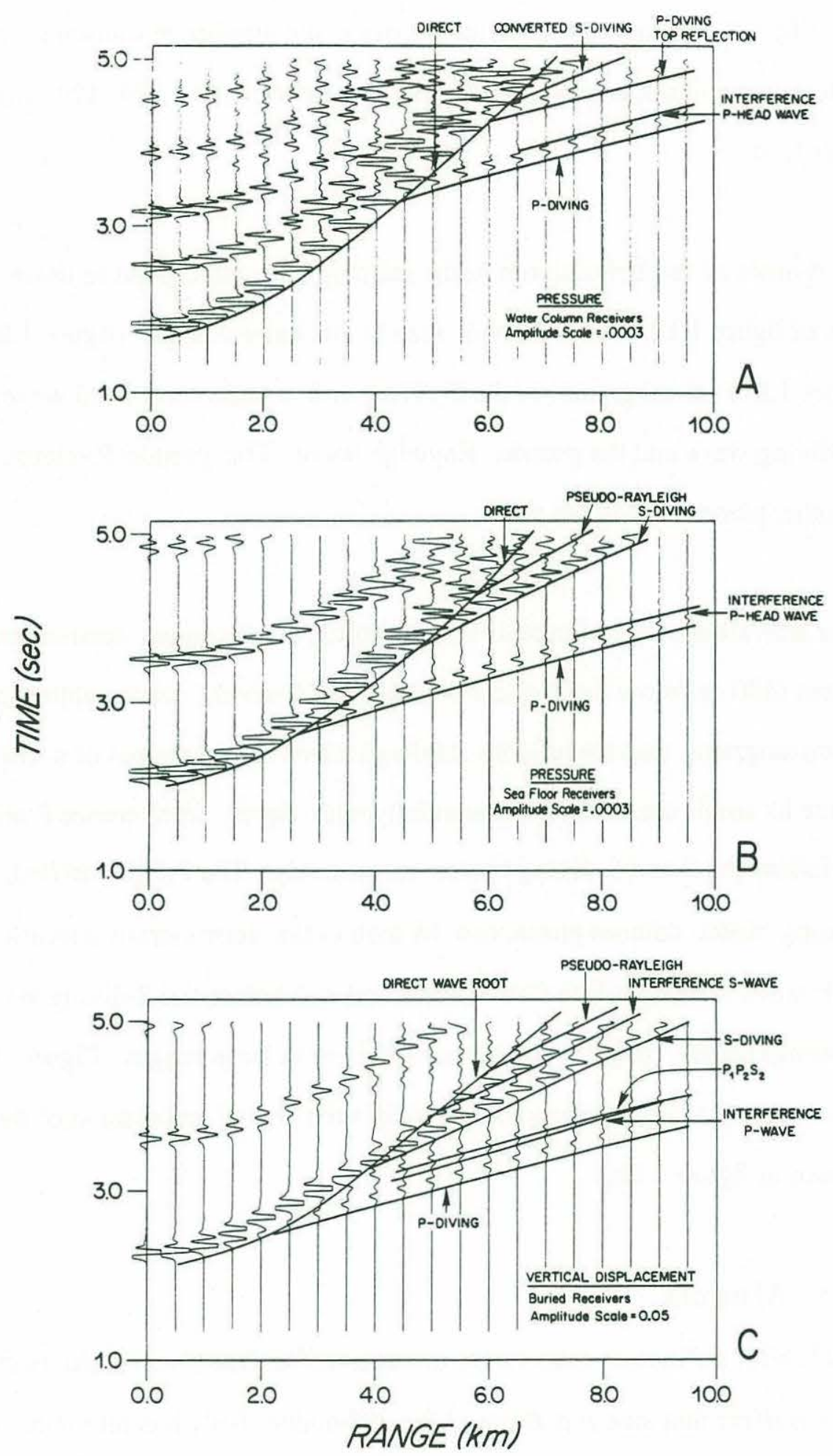
receivers (figure 1.1lb) record only water waves (direct and multiples) incident from above but the receivers in the water column (figure 1.11a) record both up and downgoing waves (direct and multiples). Therefore, figure lla has twice the number of multiples of 1.llb. As in the snapshots, these multiples are reflections from the top of the grid rather than from the true sea surface.

Ranges and times of the arrivals seen in the seismograms correspond to those seen in the snapshots of figure 1.10. Major arrivals seen in the water column (figure 1.lla) and seafloor (figure 1.llb) seismograms are the P-diving and interference head waves, the converted S-diving wave and the pseudo- Rayleigh wave. The pseudo-Rayleigh wave looks like another plane wave in the water.

A few other arrivals appear in figure 1.llc, the vertical displacement seismograms for buried receivers ( $480 \mathrm{~m}$ below the seafloor). The P and S-diving waves appear as they do in the other seismograms, and the pseudo- Rayleigh wave is present but of a low amplitude since its amplitude decays exponentially with depth. Interference P and S waves immediately follow the $\mathrm{P}$ and $\mathrm{S}$-diving waves respectively. The $\mathrm{P}_{1} \mathrm{P}_{2} \mathrm{~S}_{2}$ arrival, which has no corresponding water column phase, can be seen in the seismogram arriving after the interference P-wave (at ranges less than about $8 \mathrm{~km}$ ) and before the S-diving wave. It becomes indistinguishable from the interference $\mathrm{P}$ wave at large ranges. Figure 1.1lc also contains arrivals from the direct wave root, which is the crustal expression of the direct water wave seen in figure $1.11 \mathrm{~b}$.

\section{Diffraction Models}

Five models with diffractors were run to determine if diffractions would occur, and if so, to look at the effect that size and shape of the diffracting body has on the diffractions. The anomalous bodies (figure 1.12) were placed at a range of five kilometers. This gives 

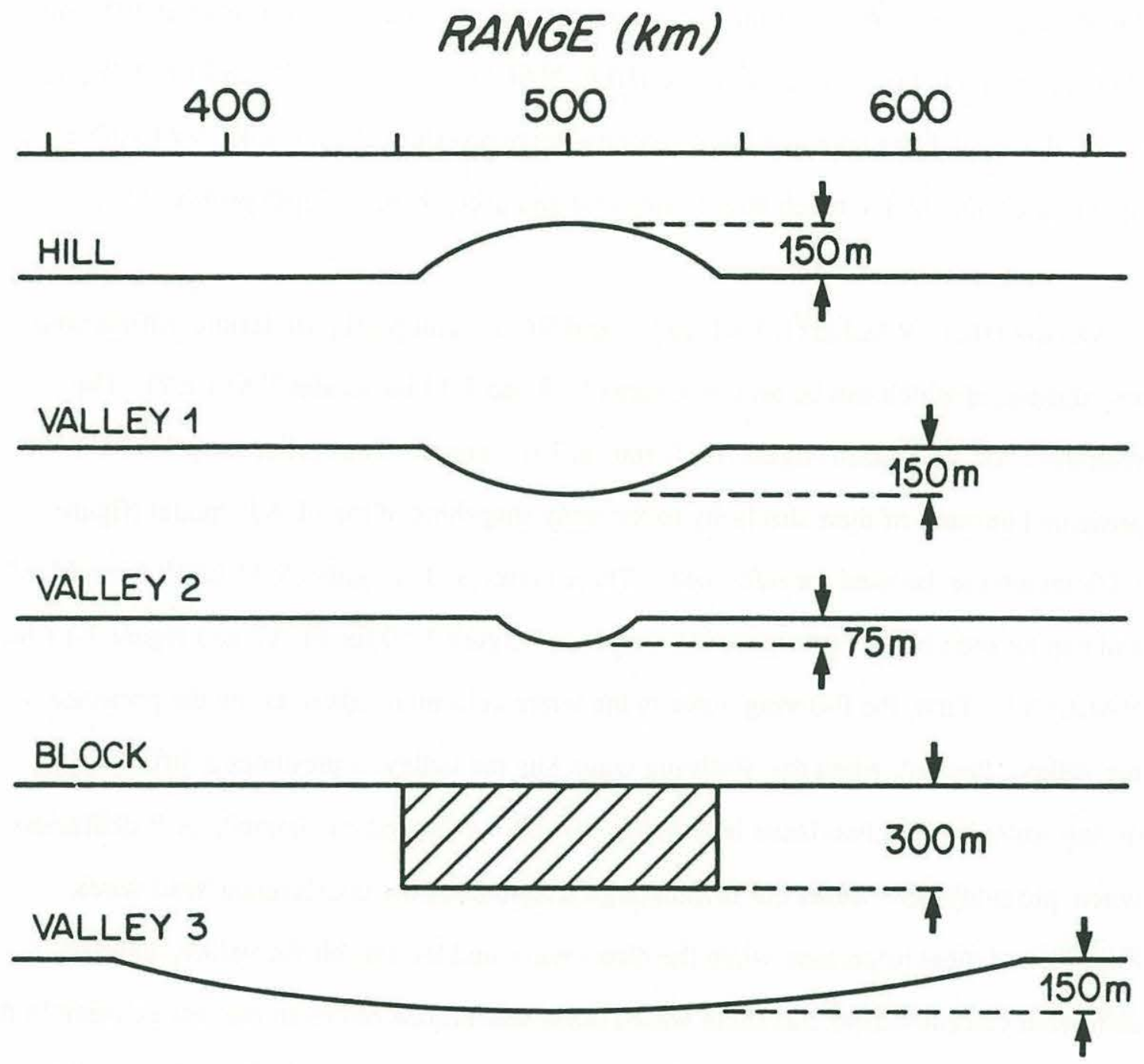

Figure 1.12. Relative sizes and shapes of different diffractors used for the various models. 
the seismic energy sufficient time to partition in order to examine the effects of different phases hitting the body. Four models, HILL, VALLEYl, VALLEY2, and BLOCK, had relatively small diffractors and used velocity-depth profile A (figure 1.4). VALLEY3, the final model run, had a much larger diffractor and used velocity-depth profile B.

Models HILL, VALLEY1, VALLEY2, and BLOCK all produced definite diffractions, an example of which can be seen in figures 1.13 and 1.14 for model VALLEYl. The snapshots for VALLEYl (figure 1.13) start at 3.6 seconds. The earlier snapshots are not presented because of their similarity to the early snapshots of the FLAT model (figure 1.10, which can be used for reference). Three features distinguish VALLEYl from FLAT and can be seen in the corresponding snapshots (figure 1.10 for FLAT and figure 1.13 for VALLEYl). First, the P-diving wave in the water column is distorted by the presence of the valley. Second, when the P-diving wave hits the valley, it produces a diffracted Sdiving wave in the crust (seen best in the 4.0 and 4.4 second snapshots). A P-diffracted wave probably also occurs but is indistinguishable from the interference head wave. Finally, and most important, when the direct wave and its root hit the valley, definite diffracted compressional and shear waves occur and radiate out from the valley (seen in the 4.0 and 4.4 second snapshots). The seafloor seismogram for VALLEYl (figure 1.14) shows all of the arrivals seen in the FLAT model (figure 1.11) as well as the S-diffracted arrival (from P-diving) and the P-diffraction (from the direct wave). The S-diffraction set up by the direct wave is not distinguishable from the pseudo-Rayleigh wave in the seismogram. Although the snapshots and seismograms for models BLOCK, HILL, and VALLEY2 are not presented here, the same three effects are present in all of these models.

VALLEY3 failed to produce a significant diffraction (see figure 1.15). The expected P-diving and interference head waves and the S-diving wave are all seen in this model as in the FLAT model. The P-diving wave is again distorted by the presence of the 
valley but no significant diffractions occur either when the P-diving or the direct wave hit the valley. Because the upper crustal velocities are lower in this example (V-Z function $B$ ) than in the previous examples (V-Z function $\mathrm{A}$ ), the P-diving and interference waves are much more separated.

Figure 1.16 shows the relative amplitudes of the diffracted arrivals and other phases for the seafloor trace at 7 kilometers range of all six models. Using the trace from the FLAT model for reference, it is obvious that the shape of the diffractor is very important in determining the character of the signals. VALLEY1 and VALLEY2 have almost identical signals except for slight differences in amplitudes. The BLOCK and HILL traces are different from each other and from the VALLEY1 and VALLEY2 models. The trace from VALLEY3 is different from all of the others not only because of the size of the valley but also because it was produced with a different velocity-depth function. All of the models which contain significant diffractions have the same arrivals, but often with different arrival times. The amplitudes of the diffractions, as well as most of the other arrivals, are of the same magnitude from one model to the next. The only phase which varies appreciably between the models is the interference head wave which appears to be significantly affected by topography. 
Figure 1.13. Divergence and curl of displacement snapshots for the VALLEYl model (corresponding to compressional and shear energy). Earlier timesteps are very similar to those of the FLAT model. Distortion of the P-diving wave can be seen at 3.6 seconds. The diffracted phases from the P-diving wave and the direct wave begin to appear in the 4.0 second snapshot and are quite clear as separate phases at 4.4 seconds. 


\section{VALLEY1 MODEL}
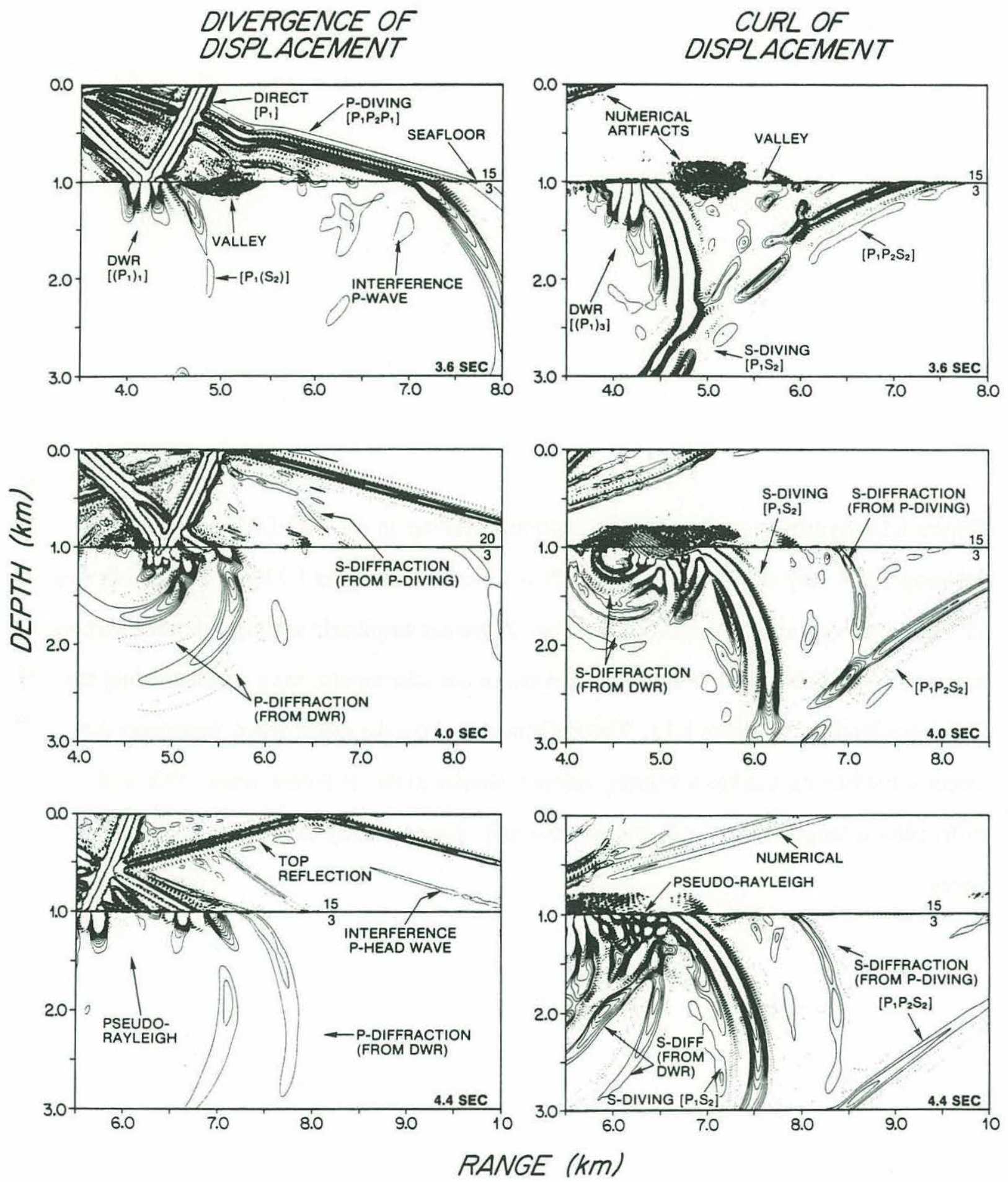
Figure 1.14. Synthetic seismogram for seafloor receivers in the VALLEYl model. The seismogram is very similar to that of the FLAT model (see figure 1.11) except for the two diffracted arrivals and the amplitude scaling. A greater amplitude scaling was used here to accentuate the diffracted arrival. Other arrivals in the seismogram have approximately the same amplitude as in figure 1.11. The P-diffraction from the direct wave intercepts the direct wave branch and has a velocity (slope) similar to the P-diving wave. The P-S diffraction is tangential to the P-diving wave and has a velocity similar to the S-diving wave. 


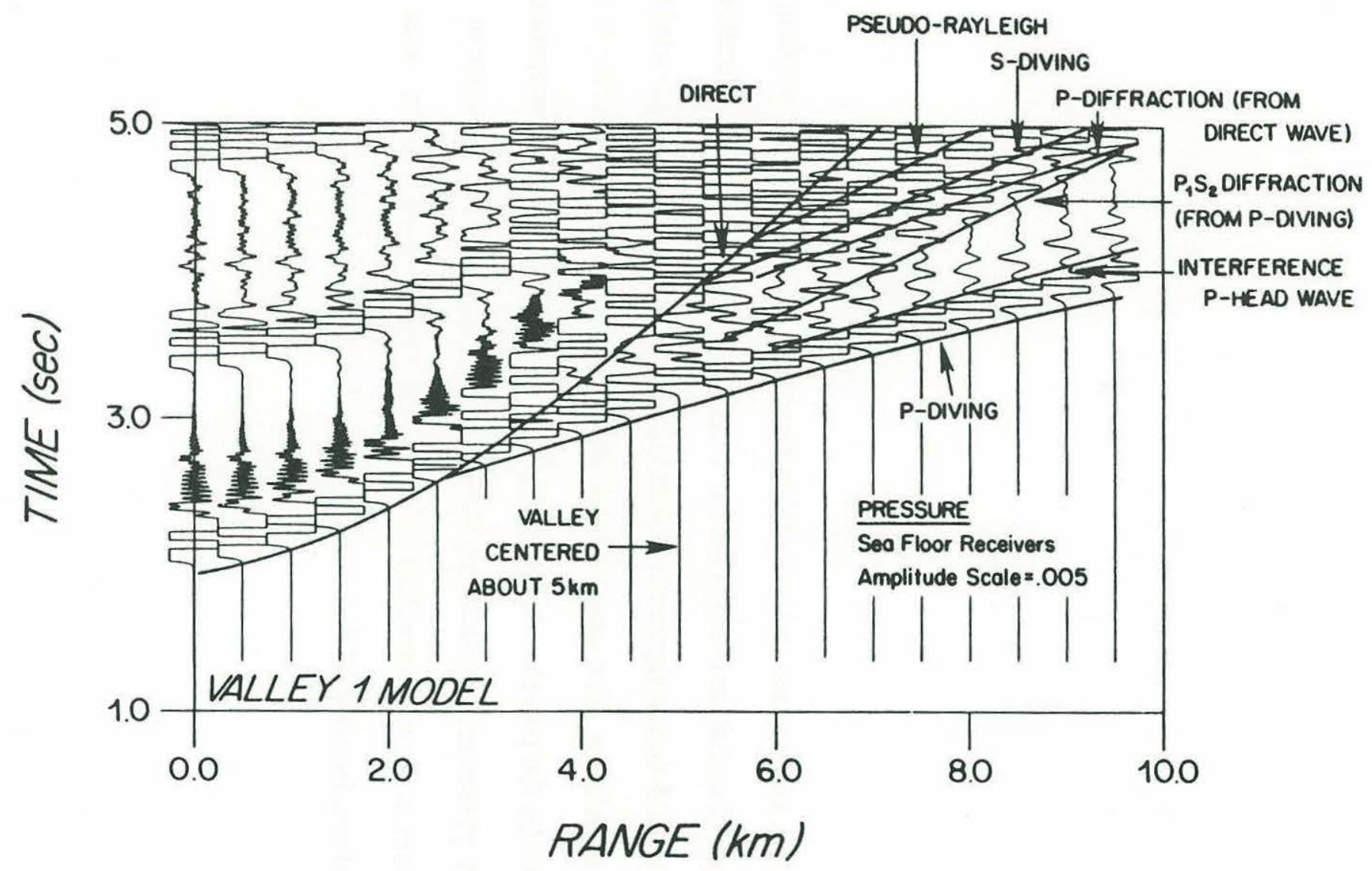


Figure 1.15a. Divergence and curl of displacement snapshots (corresponding to compressional and shear energy) for the model VALLEY3. Amplitude scaling of these snapshots is 0.5 that of figures 1.10 and 1.13 . Note also that the range window moves with the wavefronts. The water column plane wave corresponding to the P-diving wave is distorted by the presence of the valley in the 3.6 and 4.0 second snapshots. However, diffracted phases are indiscernible in any of the snapshots. The presence of prominent Swave phases in the curl snapshots indicates that strong P-S conversion takes place with the velocity-depth function of this model (profile B of figure 1.4). 


\section{VALLEY 3 MODEL}

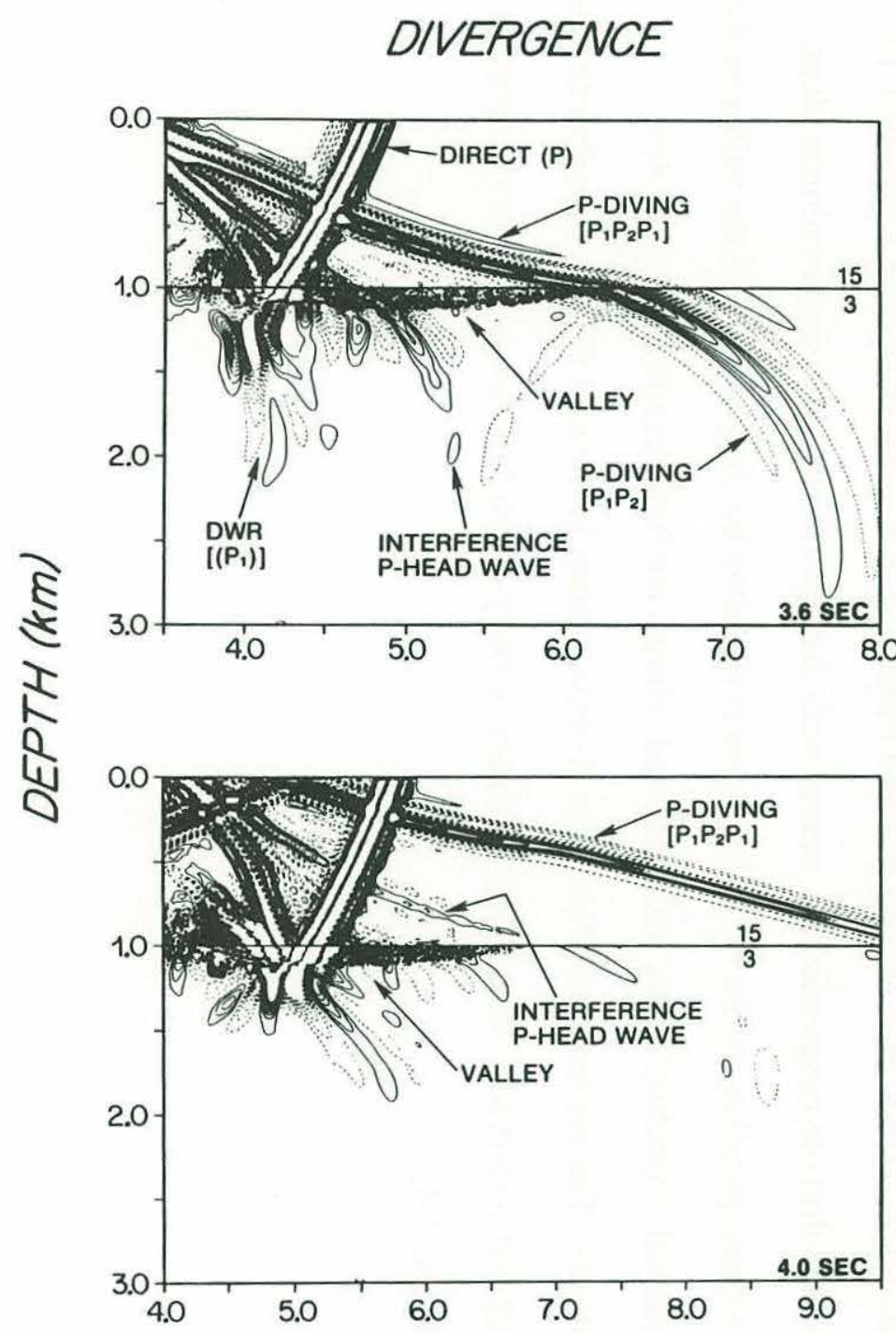

CURL
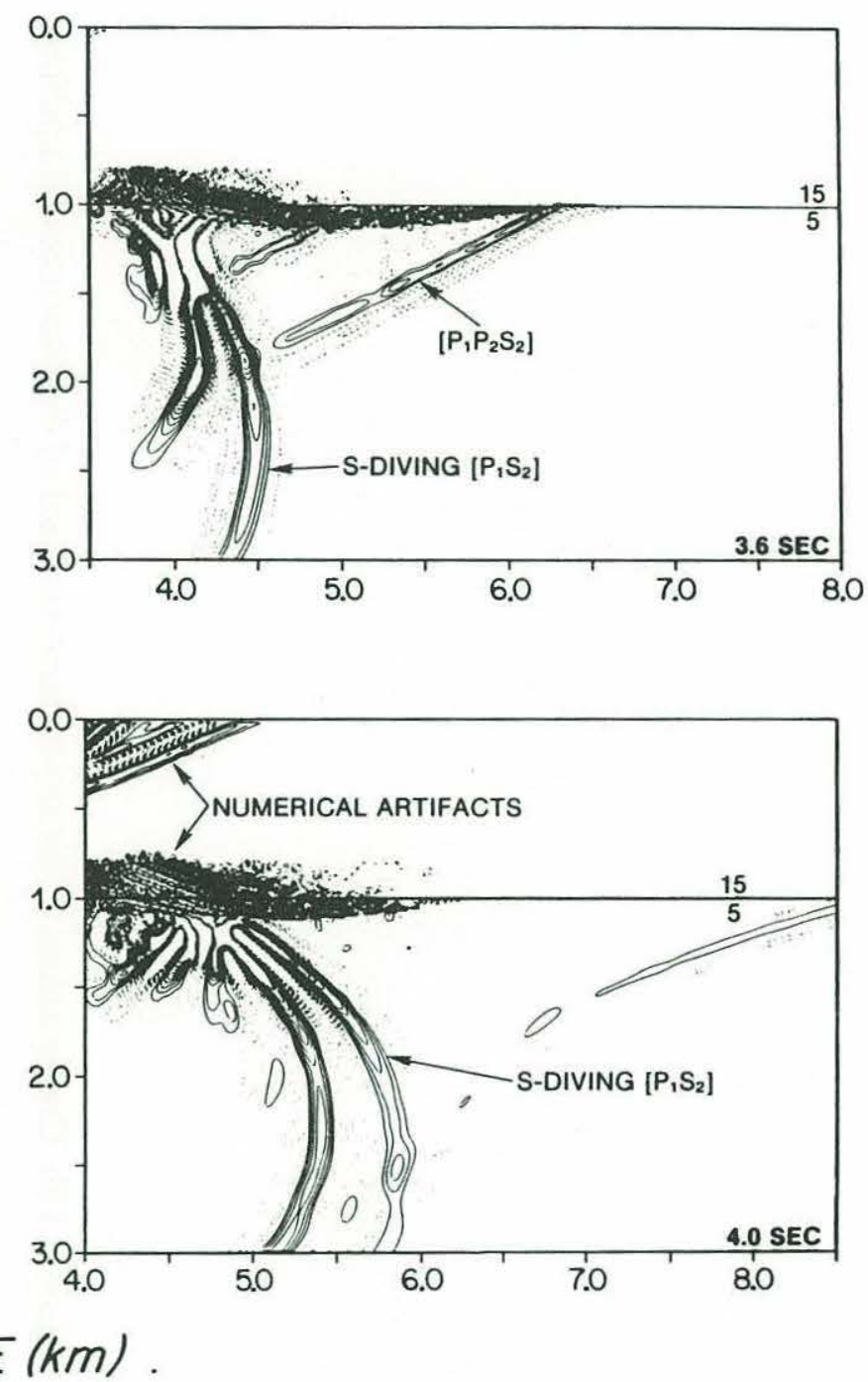
Figure 1.15b. Continuation of the divergence and curl of displacement snapshots (corresponding to compressional and shear energy) for the model VALLEY3 as seen in figure 1.15a. Amplitude scaling of these snapshots is 0.5 that of figures 1.10 and 1.13. Note also that the range window moves with the wavefronts. The water column plane wave corresponding to the P-diving wave is distorted by the presence of the valley in the 4.4 second snapshot. However, diffracted phases are indiscernible in any of the snapshots. The presence of prominent S-wave phases in the curl snapshots indicates that strong P-S conversion takes place with the velocity-depth function of this model (profile B of figure 1.4). 


\section{VALLEY 3 MODEL}
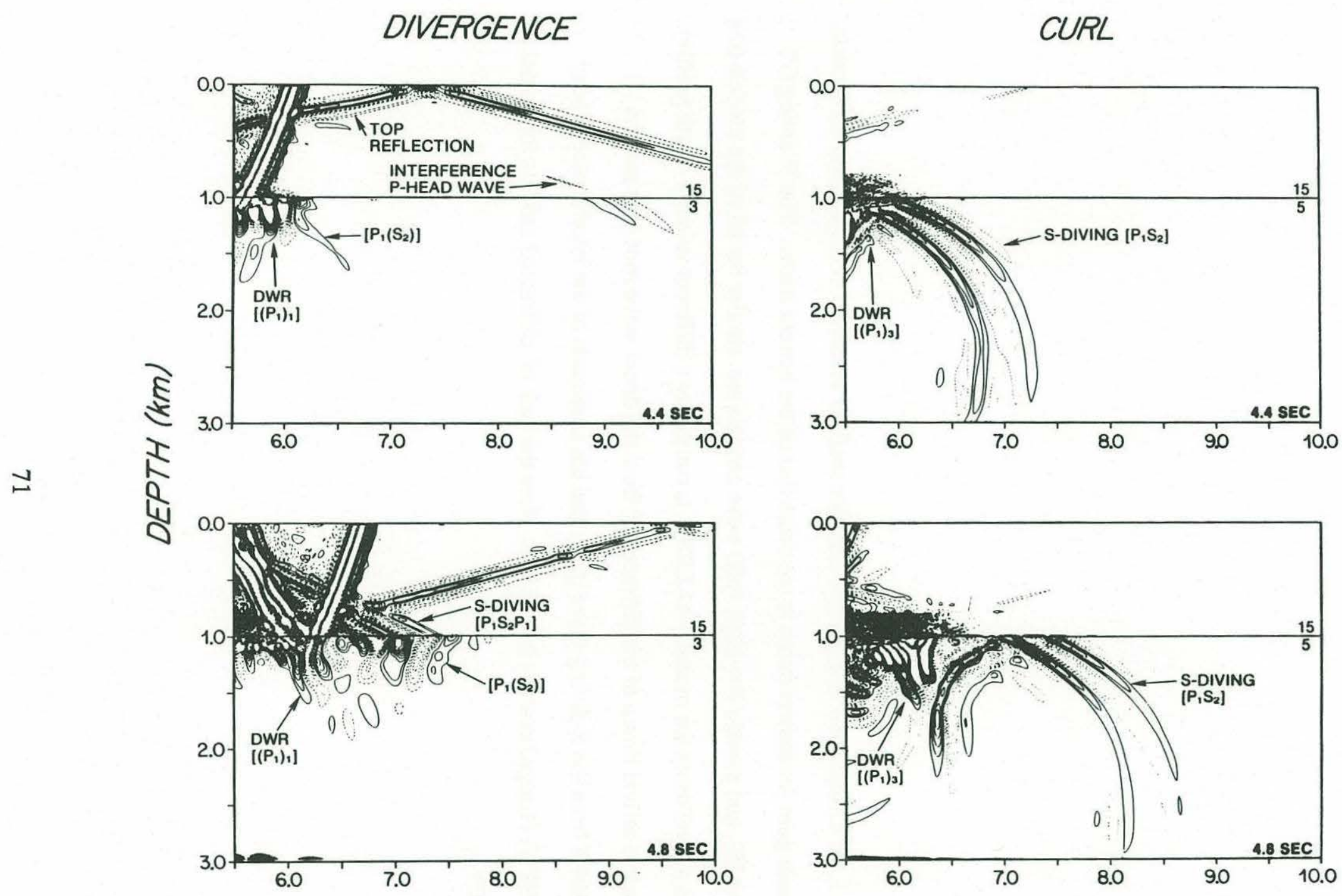

RANGE (km) 
Figure 1.16. Comparison of the $7 \mathrm{~km}$ trace for seafloor receivers in each of the six models. Amplitude gain for the top traces is ten times that of the bottom traces. The P-diving $(\mathrm{P})$, S-diving (S), and pseudo-Rayleigh ( $\mathrm{pR}$ ) wave arrivals are similar for all of the models (the delay in arrival times for model VALLEY3 is caused by a different velocity-depth profile). The relative arrival times of the diffracted $\mathrm{P}$ from the direct wave root $(\mathrm{dP})$ and the diffracted S from the P-diving wave (dS), and the amplitude of the interference P-head wave (IHW) change between the models. Note the lack of diffracted arrivals for model VALLEY3. 

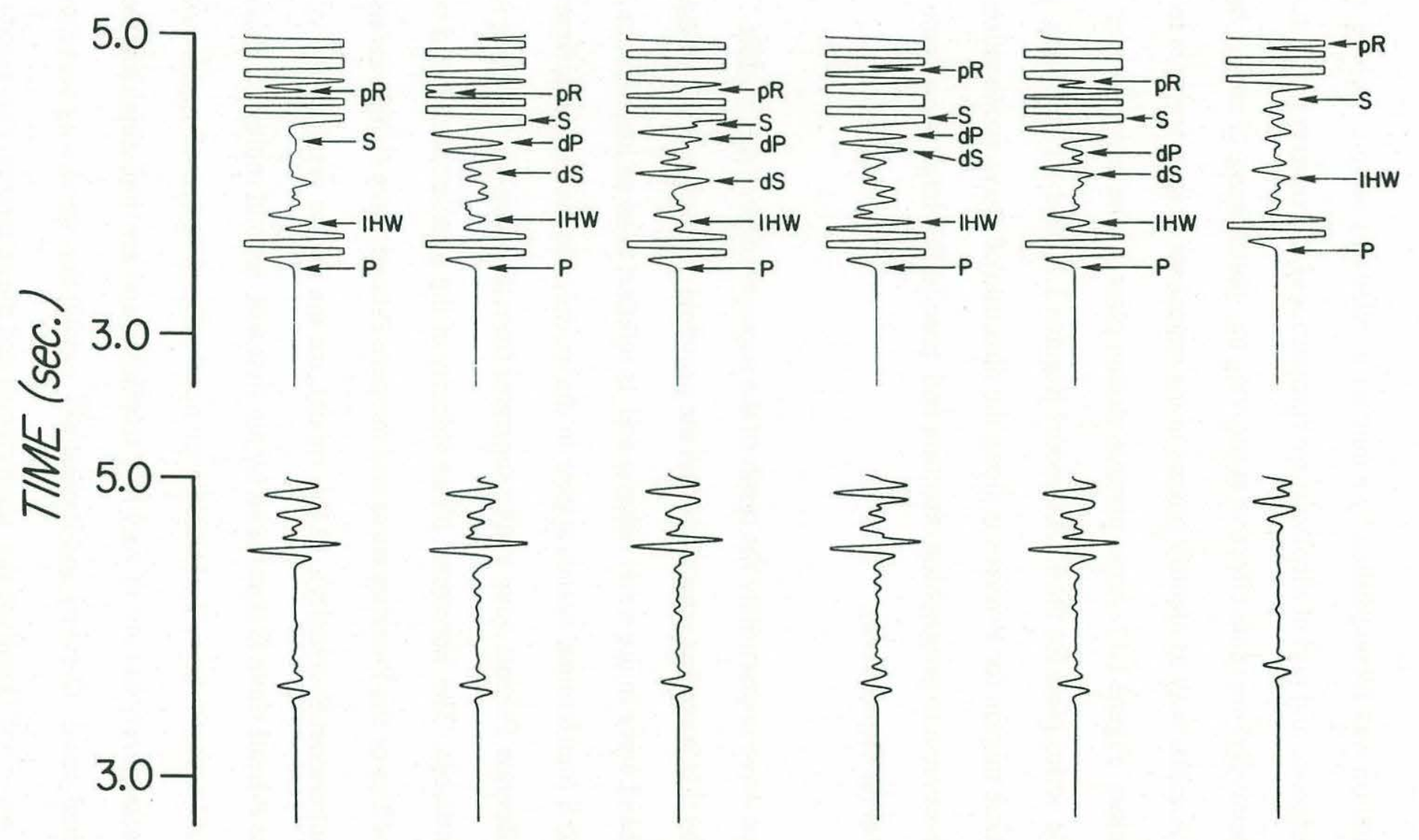

FLAT HILL VALLEY 1 BLOCK VALLEY 2 VALLEY 3 


\section{DISCUSSION}

Phase identification was accomplished by a number of relatively simple techniques. Snapshots of divergence and curl of displacement (proportional to compressional and shear energy respectively) are quite effective at showing the partitioning of energy between $\mathrm{P}$ and $\mathrm{S}$ waves. Another way to identify phases in the snapshots or time series is to plot their particle motion. Figure 1.17 shows particle motion plots for the major phases present in the time series plots for the FLAT model (figures 1.10 and 1.11). These plots confirm that particle motion for $\mathrm{P}$-waves is along the direction of wave propagation, Swave motion is transverse to propagation direction and pseudo-Rayleigh wave motion is retrograde elliptical (at the seafloor).

The interference wave is essentially the result of the superposition of all possible multiples ${ }^{18}$. When this wave has separated from the principle diving wave it appears similar to a pure head wave in the water column and is referred to as an interference head wave. Both $\mathrm{P}$ and $\mathrm{S}$ interference waves appear in the models shown here (see figure 1.10) but only the interference P-head wave totally separates from the principle P-diving wave in the time range available. The horizontal phase velocity of the interference P-head wave is between the velocities of the P-diving wave and the pure P-head wave (which exists for a half-space with uppermost P-velocity). In this model, for the ranges present, the velocity of the interference P-head wave is dominated by the first and second multiple. From ray theory, these should separate from the interference head wave at ranges of approximately 6.5 and $8.5 \mathrm{~km}$ respectively but are of very low amplitude and are indistinguishable from the interference head wave. Cerveny and Ravindra 18 predict that the diving wave and the interference head wave will dominate the seismogram and the separated, non-interference multiple diving waves will be weaker. Our results support the predictions of Cerveny and 
Ravindra. However, the velocity gradients used for this study ( $4.0 \mathrm{sec} .^{-1}$ at the seafloor) are much greater than the those used by Cerveny and Ravindra $\left(\leq 0.04 \mathrm{sec} .^{-1}\right)$. Data from $\mathrm{OBH} 2 \mathrm{~W}$ lack any interference waves, because topography along the line causes attenuation due to scattering. Indeed, the amplitude and coherence of the interference Phead wave arrival in the HILL model is decreased from the amplitude in the FLAT model simply by adding a single hill onto the sea floor (compare figures 1.10 and 1.18).

Stephen 7 discussed the generation of 'double head waves' due to interaction with a seafloor hill. In this example the compressional wave critical angle is reached twice by a direct wave travelling over the hill (once on the slope of the hill and again on the flat surface after it). The situation is somewhat more complicated when a velocity gradient is present since diving waves rather than head waves occur and there is no single critical angle for diving waves. Double head waves do not, however, occur in the models of this study, because the anomalous structures were placed far beyond the critical ranges for any diving compressional energy. However, when the P-diving wave hits the diffractor, it causes a converted P-S diffracted phase to occur. Since this phase occurs in the BLOCK, as well as the HILL and VALLEY models, it appears that it must be a diffraction rather than some sort of 'double head wave' refraction.

The occurrence of backscattered energy also verifies the hypothesis that diffraction rather than refraction or reflection of seismic energy is occuring around the seafloor features. Backscattering of energy is most convincingly seen in figure 1.19 , the time series of pressure for a buried receiver in the BLOCK model. Arrivals predicted by the flat model, as well as the P- diffraction (from the direct wave) are all present in the seismogram. Since this is the pressure time series, S-waves do not have large amplitudes (compare this with the vertical displacement seismogram for buried receivers of the FLAT 
Figure 1.17. Particle motion plots for major arrivals of the FLAT model. The large arrow indicates the direction of wave propagation and the cross indicates the onset of motion for each plot. Receivers for 1.17a-e are buried at $480 \mathrm{~m}$ below the seafloor and for $1.17 \mathrm{f}$ the receivers are at the seafloor. The P-diving wave (1.17a) and the interference P-wave (1.17b) both have particle motion parallel to propagation direction. The S-diving wave (1.17d), $\mathrm{P}_{1} \mathrm{P}_{2} \mathrm{~S}_{2}$ (1.17c), and the interference $\mathrm{S}$-wave (1.17e) all have particle motion perpendicular to propagation direction and the pseudo-Rayleigh wave (1.17f) has retrograde elliptical particle motion at the seafloor. All of the plots are for receivers at a range of $7 \mathrm{~km}$. 

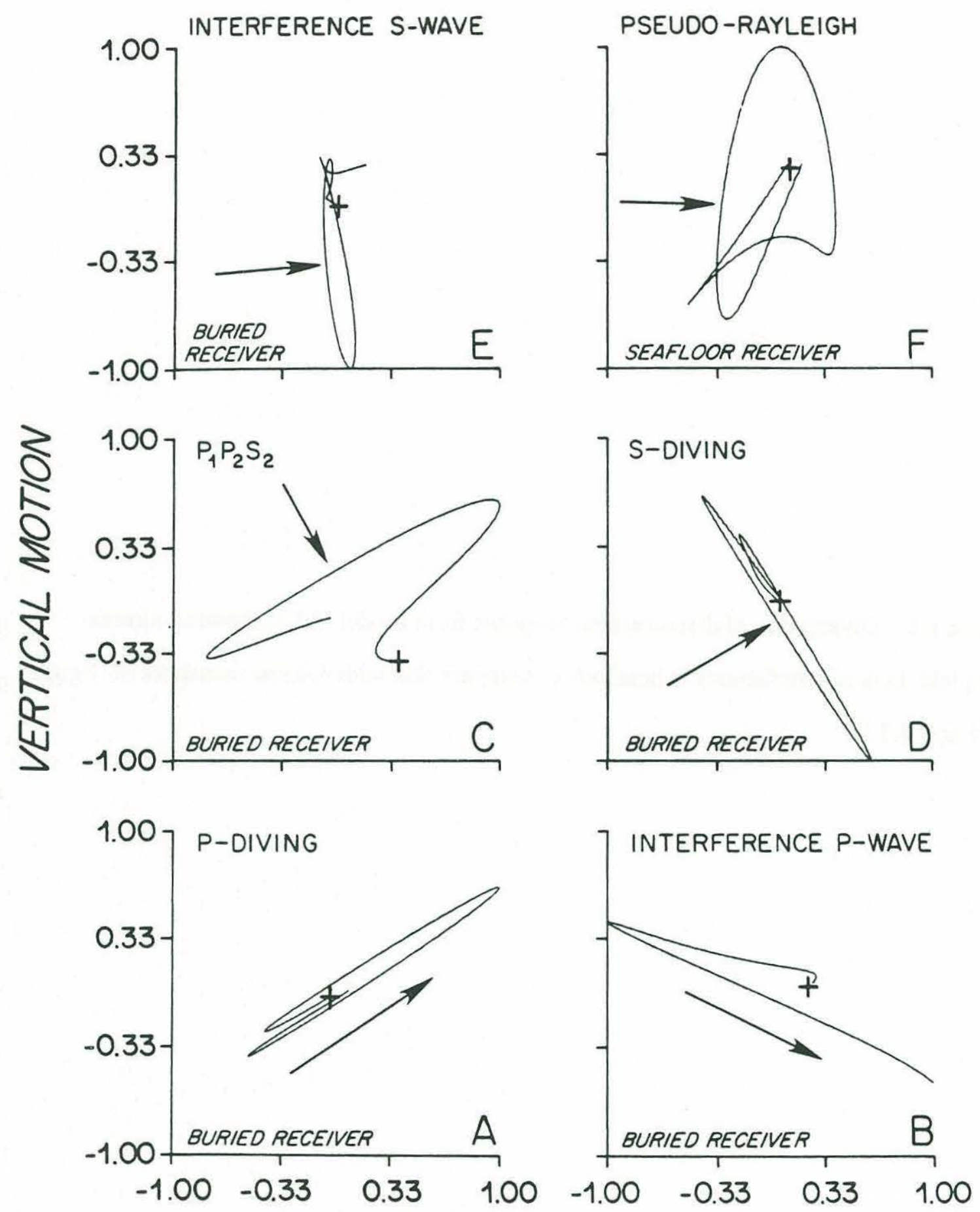

HORIZONTAL MOTION 
Figure 1.18. Divergence of displacement snapshot from model HILL showing almost complete lack of interference P-head wave (compare this with $4.4 \mathrm{sec}$ snapshots of figures 1.10 and 1.13). 
HILL MODEL DIVERGENCE AT 4.4 SECONDS

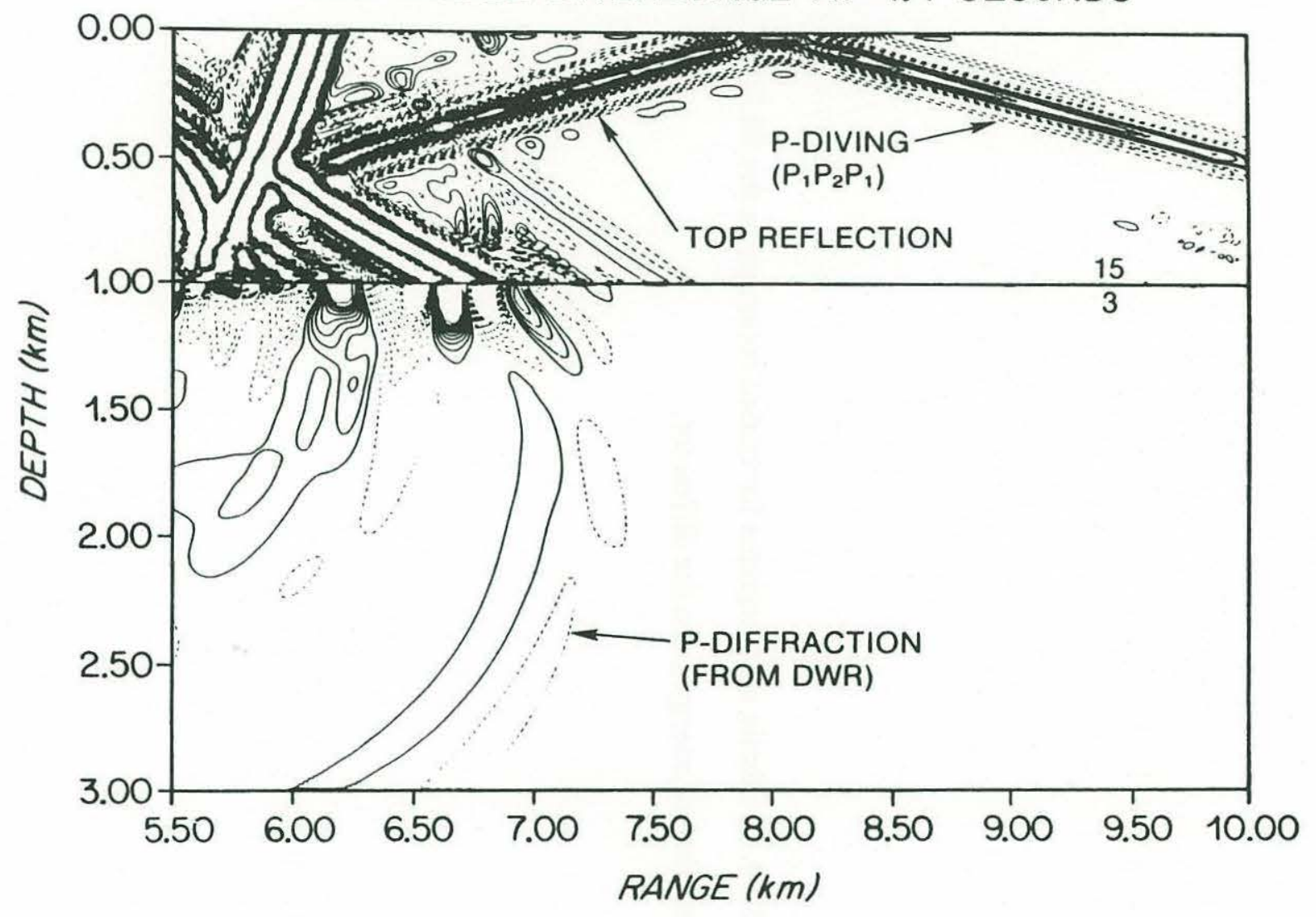


Figure 1.19. Synthetic seismograms for buried receivers in the BLOCK model showing backscattering of energy from the diffractor. 


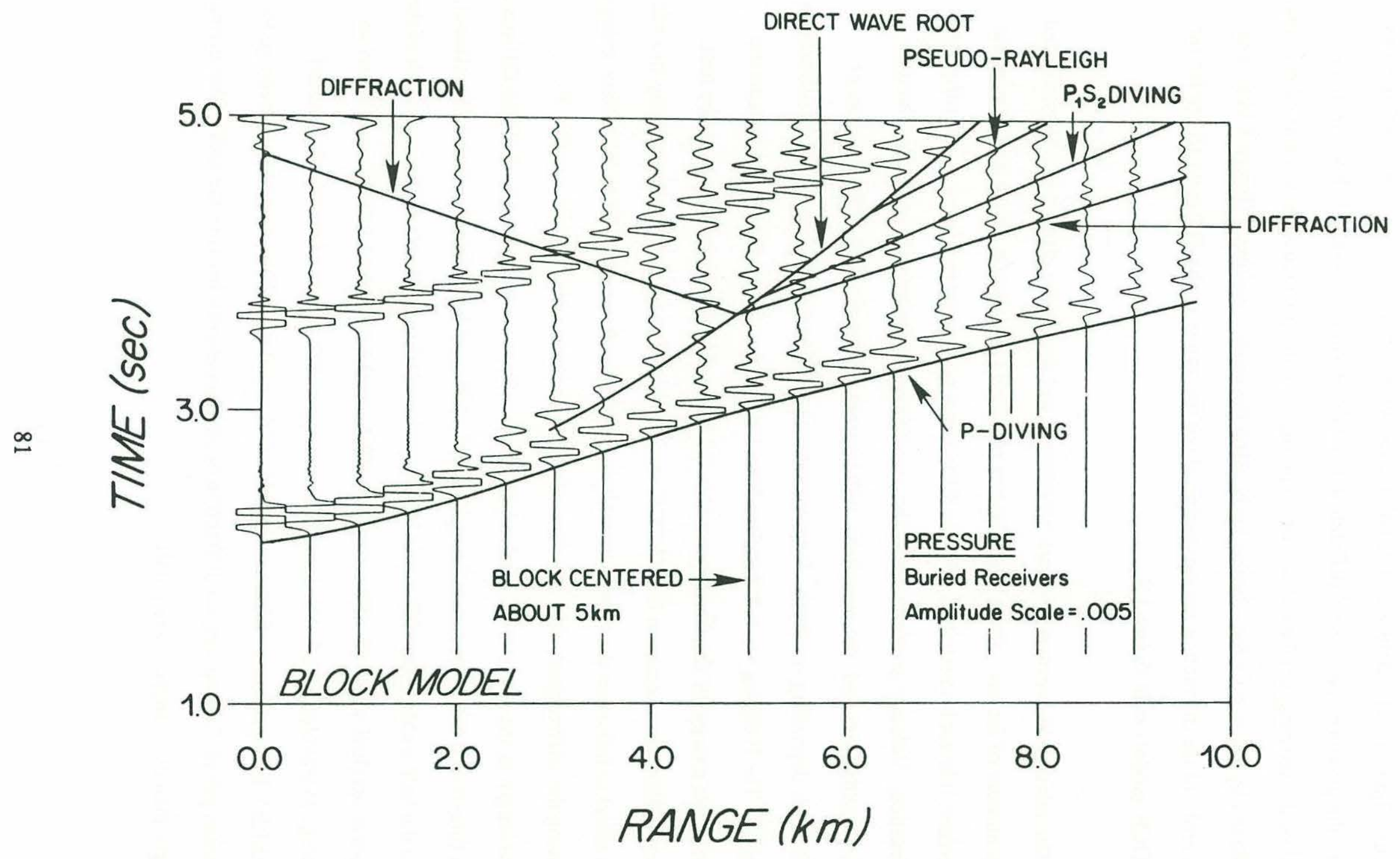


model, figure 1.llc). Only the pressure from $\mathrm{P}$-waves coupled to the $\mathrm{S}$-waves will show up in the pressure plot. The S-diffraction from the P- diving wave is below the noise level of the seismogram for this reason. The signal of the diffraction is quite similar to that predicted by ray tracing for a buried body using the model geometry (figure 1.9b). The two limbs of the diffraction are symmetric about the range of the diffractor $(5 \mathrm{~km}$ for the BLOCK model) as in figure 1.9.

The relationship between seismic wavelength and size of the diffractor is complicated by a number of factors. Since the diffrators modeled in this study are at the water-crust interface, it is not immediately obvious if the direct or transmitted energy is causing the diffraction. Velocity gradients cause the wavelength of the seismic energy to change rapidly with depth and the wavefront will interact differently with different sizes of diffractors depending on depth. It appears that the diffractions are the result of subsurface energy. The P-diving wave is incident from below when it hits the diffractors and the subsurface energy for the direct wave is in the form of its prominent direct wave root. Also, a diffraction occurs in the BLOCK model which has no surface topography (no hill or valley) to influence the energy actually travelling in the water. If it is subsurface energy causing the diffractions, then the sizes of the diffractors must be similar to the Pwavelength in the upper crust. Indeed, if the wavefront sees only one half of the diffractor at a time (i.e. it first encounters the upslope of a hill anad later encounters the downslope), then the half width of the diffractors is almost identical to the average P-wavelength of the upper crust (half width of 450 meters for HILL, VALLEYl and BLOCK models and an average P-wavelength of 400 meters for the upper 400 meters of crust). For model VALLEY3, which has a diffractor of half width equal to 1320 meters and an average Pwavelength of 320 meters, no diffraction occurs apparently because the half width is much larger than the seismic wavelength. 
The possibility of diffractions occuring from a number of different anomalous bodies has been clearly established. On one hand, the smaller diffractors of HILL, BLOCK, VALLEY1, and VALLEY2 caused diffractions but are generally not seen in the corresponding bathymetry (figure 1.4). On the other hand, VALLEY3, a closer representation of the bathymetry of the data area, does not cause a diffraction. One possible compromise to the problem is that the hill offset behind the valley in figure 1.4 could be the source of the diffracted energy. Because it is offset behind a large valley its relative size is closer to the size of the diffractors in the earlier models. The other hills in the section are too low and broad to act as diffractors.

Assuming that the offset hill can be the source of the diffracted energy, a number of comparisons between the data and the models can be made. If the difference in geometry between model and data is considered, the travel times for the diffraction in the models (HILL, VALLEY1, VALLEY2, and BLOCK) are approximately the same as those in the data. The amplitudes of the diffracted arrivals, while not as large as the first P-refraction arrival, are significant. It is interesting that in the data, the diffraction is even larger in amplitude than the P-diving wave for some ranges. Presumably, both the travel times and amplitudes of the model diffractions could be altered by perturbation of the model velocity-depth functions.

Direct comparison of the data and models is not possible because of differences in geometry and source signatures for the two setups. We stress that the objective of this study was to demonstrate the phenomenon of refraction branch diffraction, and not to match exact waveforms in the data. Simple ray tracing showed that movement of the diffractor did not significantly change the shape of the diffracted arrival. We chose to place the diffractors at $5 \mathrm{~km}$ range in the models to best take advantage of the finite difference grid without regard to the exact matching of any one data trace geometry. 
The signatures of the synthetic and airgun sources are different enough to make waveform matching between the models and data unreasonable. The synthetic source is a single pulse with peak frequency at $10 \mathrm{hz}$ (see figure 1.20). Airgun sources typically have an initial pressure pulse plus a number of secondary bubble pulse reverberations. A single pulse source was used for the modeling in order to better define and identify the diffracted arrival. The exact arrival of the refraction branch diffraction in any one data trace is often masked by the airgun source signature of the refraction branch of the seismogram (figure 1.1). Only when the entire survey of $\mathrm{OBH} 2 \mathrm{~W}$ is taken as a whole does the refraction branch diffraction stand out as a coherent arrival. The synthetic source used for the finite difference modeling allows the diffracted arrival to be picked much more easily on any single trace (figure 1.16).

A complicating factor which occurs in the models but not in the data is the appearance of shear and pseudo-Rayleigh waves. Direct wave energy lost to shear energy by P-S conversion could potentially be a source of the additional energy needed to increase the diffraction amplitudes. White and Stephen 20 , showed that P-S conversion is decreased by increasing the Poisson's ratio of the basement or by increasing the impedance contrast between the S-wave in basement and the $\mathrm{P}$-wave velocity in the overlying layer. Spudich and Helmberger 21 produced two ocean bottom models which do not contain shear waves, one which has a crust of high Poisson's ratio (ratio of 0.38 rather than 0.25 for a Poisson solid) and the other which had a high impedance contrast at the top of the basement (crustal $\mathrm{S}$-wave velocity of $2.023 \mathrm{~km} / \mathrm{sec}$ and sediment $\mathrm{P}$-wave velocity of $3.0 \mathrm{~km} / \mathrm{sec}$ ). The young crust in the ROSE area, however, is unsedimented and has water directly on top of basement. It is not clear how the substitution of a water layer above the basement for the Spudich and Helmberger transition zone or White and Stephen sediment layer would affect P-S conversion at the interface. 
From the modeling done in this study it is clear, however, that simply lowering the upper crustal P-wave velocities, while assuming a Poisson solid, does not eliminate P-S conversion (conversion occurred for both velocity-depth functions of figure 1.3). A more realistic model of crustal Poisson's ratios (rather than assuming a uniform Poisson's ratio of 0.25 ) would be one with some depth dependence caused by greater fracturing and porosity of the crust at shallow depths. A Poisson's ratio closer to 0.35 would be more realistic for young, unsedimented crust found in this part of the ROSE area. Other factors which affect the appearance of S-waves in marine seismic data are; angle of incidence of the P-wave, incoherency of S-arrivals due to rough topography and lateral crustal heterogeneities, and absorption of shear energy (higher absorption in fractured, porous crust).

The strength of P-S conversion, while it does not fit with the data, is fortuitous in that it demonstrates the pseudo-Rayleigh wave at large times and ranges (see figures 1.10 and 1.17). There is a definite break between the shear and pseudo-Rayleigh waves and the amplitudes of the two are comparable (figure 1.16). Ray parameter analysis on the wavefront shows that it is travelling with a velocity of $2.0 \mathrm{~km} / \mathrm{sec}$. If the pseudo-Rayleigh wave travels at $92 \%$ of the shear wave velocity (the free surface Rayleigh wave velocity relationship ${ }^{1}$ ) then this wave corresponds to an S-wave of velocity $2.17 \mathrm{~km} / \mathrm{sec}$ at an effective depth of 145 meters instead of being affected by the interface S-wave velocity of $1.85 \mathrm{~km} / \mathrm{sec}$. Rather than travelling at the velocity right at the interface, the velocity gradient causes the pseudo-Rayleigh phase to travel at an 'effective' velocity which is related to the seismic wavelength and the shear velocity gradient. 
Figure 1.20. Normalized pressure signatures of the synthetic and airgun array sources. The synthetic source is the third derivative of a Gaussian curve and has a peak frequency of $10 \mathrm{hz}$ with an upper half power frequency of $13.5 \mathrm{hz} 22$. The seafloor reflection pulse is from the Lamont Doherty Geophysical Observatory airgun array used for ROSE airgun line 1 (J. Diebold, pers. comm.). 


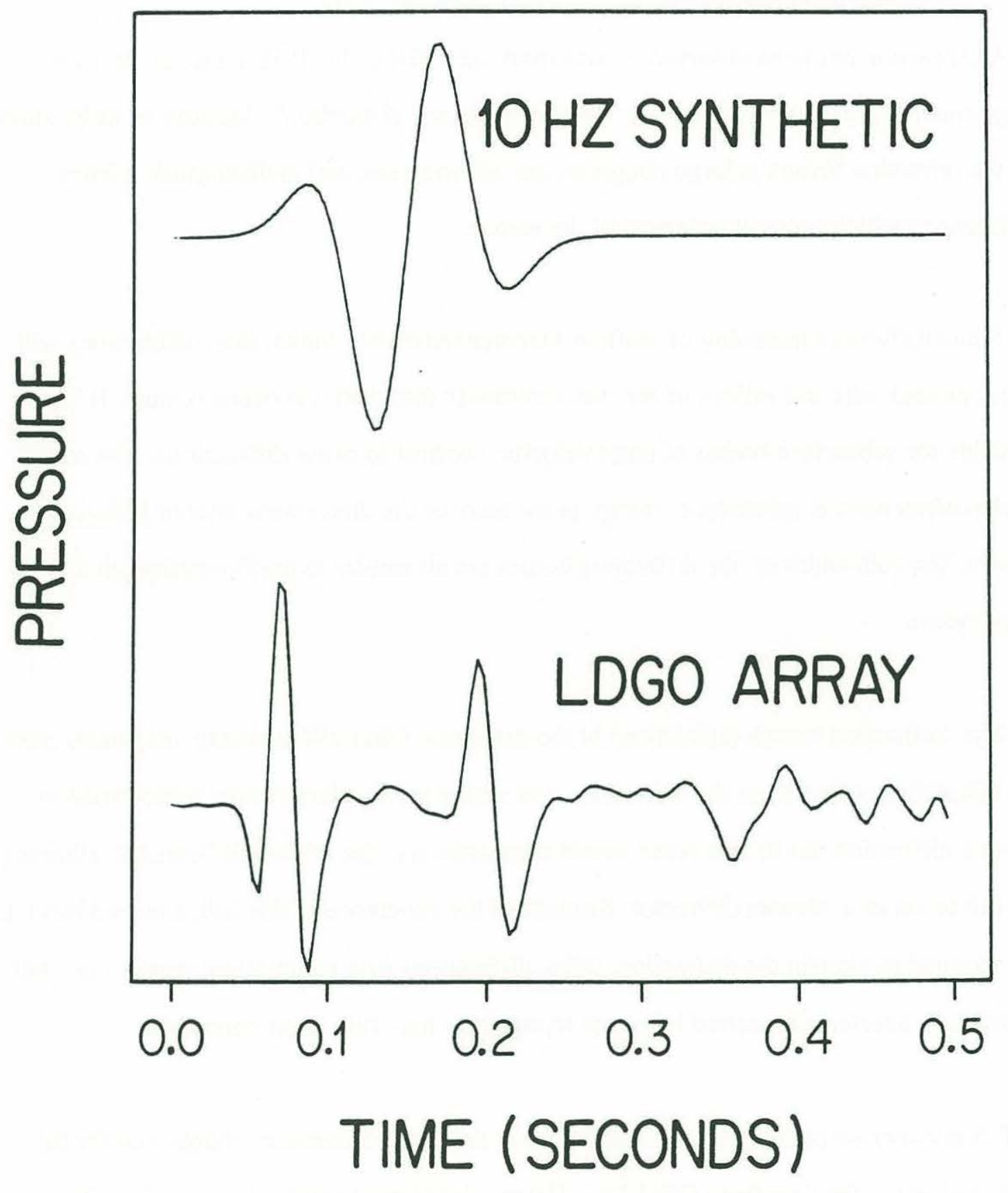




\section{CONCLUSIONS}

The following conclusions can be made from this study:

1. A diffraction has been observed in data from one $\mathrm{OBH}$ of the Rivera Ocean Seismic Experiment. We call this arrival the "refraction branch diffraction" because of its location on the refraction branch at large ranges on the seismogram, and to distinguish it from diffractions which occur at near normal incidence.

2. Finite difference modeling of seafloor heterogeneities has shown that diffractions will occur around hills and valleys of the size commonly found on the ocean bottom. It is also possible for subsurface bodies of large velocity contrast to cause diffractions. The source of the diffractions is subsurface energy in the form of the direct wave root or P-diving waves. The half-width of the diffracting bodies are all similar to the P-wavelength in the upper crust.

3. The "refraction branch diffraction" in the data from $\mathrm{OBH} 2 \mathrm{~W}$ probably originates from the hill slightly offset from the track line. The valley seen at large range is too broad to cause a diffraction but its low relief would accentuate the size of the hill behind it, allowing the hill to act as a distinct diffractor. Because of the occurence of this hill, a buried block is not required to explain the diffraction. Other diffractions may occur along airgin line 1 but destructive interference caused by rough topography may ruin their coherence.

4. P-S conversion at the seafloor is prevalent in the finite difference models run for this study but not in the data from $\mathrm{OBH} 2 \mathrm{~W}$. Using a low upper crustal P-wave velocity and assuming a Poisson's solid does not eliminate the shear conversion. If the velocity-depth function of Ewing and Purdy 5 (V-Z function B in figure 1.3) is reasonable for the upper 
crust then it is apparent that the assumption of a Poisson's ratio of 0.25 is not valid. A Poisson's ratio closer to 0.35 is proposed as more realistic for this region but more analysis needs to be done to correctly determine the depth dependence of Poisson's ratio.

5. A pseudo-Rayleigh wave occurs at large ranges using the $\mathrm{V}-\mathrm{Z}$ functions appropriate for the ROSE area and assuming a Poisson solid. The wave travels at the interface at $92 \%$ of the S-wave velocity at an 'effective' depth related to the seimic wavelength and the shear velocity gradient used (approximately 145 meters for the models with V-Z function A).

6. Interference waves, seen in the models but not in the data from $\mathrm{OBH} 2 \mathrm{~W}$, are attenuated by scattering from rough topography along the line and are below the noise level in the seismograms. 


\section{ACKNOWLEDGMENTS}

We would like to thank J.I.Ewing and G.M.Purdy for invaluable discussions on the project and for providing the ROSE data. S.T. Bolmer provided assistance in display and analysis of the finite difference models. This work was supported by the Office of Naval Research under Contract No. N00014-85-C-0001. Woods Hole Contribution Number 6370. 


\section{REFERENCES}

1. K. Aki and P.G. Richards, Quantitative Seismology, Theory and Methods (W.H. Freeman and Company, San Francisco, CA, 1980),Vol. 2, pp. 240,746-749.

2. J.I. Ewing and R.P. Meyer, "Rivera Ocean Seismic Experiment (ROSE) overview," J. Geophys. Res. 87, 8345-8357(1982).

3. K. Klitgord and J. Mammerickx, "Northern East Pacific Rise: magnetic anomaly and bathymetric framework," J. Geophys. Res. 87, 6725-6750 (1982).

4. G.M. Purdy, "The variability in seismic structure of layer 2 near the East Pacific Rise at 12 N," J. Geophys. Res. 87, 8403-8416 (1982).

5. J.I. Ewing and G.M. Purdy, "Upper crustal velocity structure in the ROSE area of the East Pacific Rise," J. Geophys. Res. 87, 8397-8402 (1982).

6. G.M. Purdy, "The correction for the travel time effects of seafloor topography in the interpretation of marine seismic data," J. Geophys. Res. $\quad 87,8389-8396$ (1982).

7. R.A. Stephen, "Finite difference seismograms for laterally varying marine models," Geophys. J.R. astr. Soc., 79, 185-198 (1984).

8. R.A. Stephen, "A comparison of finite difference and reflectivity seismograms for marine models," Geophys. J. R. astr. Soc., 72, 39-58 (1983).

9. Z. Alterman and D. Loewenthal, "Computer generated seismograms," in Computational Physics, edited by B.A. Bolt (Academic Press, New York, NY, 1972), Vol. 12, pp. 35-164.

10. L. Nicoletis, "Simulation numerique de la propagation d'ondes sismiques dans les milieux stratifies a deux et trois dimensions: contribution a la construction et a l'interpretation des sismogrammes synthetiques", Ph.D. thesis, Universite Pierre et Marie Curie, Paris, France, (1981).

11. K. Bhasavanija, "A Finite Difference Model of an Acoustic Logging Tool: The Borehole in a Horizontally Layered Geologic Medium", Ph.D. thesis, Colorado School of Mines, Golden, Colorado, (1983).

12. K. Fuchs and G. Muller, "Computation of synthetic seismograms with the reflectivity method and comparison with observations," Geophys. J. R. astr. Soc. 23, 417433 (1971).

13. K.R. Kelly, R.W. Ward, S. Treitel, and R.M. Alford, "Synthetic seismograms: A finite difference approach", Geophysics, 41, 2-27 (1976). 
14. R. Clayton and B. Engquist, "Absorbing boundary conditions for acoustic and elastic wave equations," Bull. Seis. Soc. Am. 67, 1529-1540 (1977).

15. S.E. Emerman and R.A. Stephen, "Comment on 'Absorbing boundary conditions for acoustic and elastic wave equations', by R. Clayton and B. Engquist", Bull. Seis. Soc. Am., 73, 661-665 (1983).

16. A.C. Reynolds, "Boundary conditions for the numerical solution of wave propagation problems", Geophysics 43, 1099-1110 (1978).

17. L.M. Brekhovskikh, Waves in Layered Media (Academic Press, New York, 1960), pp. 318-324.

18. V.Cerveny and R. Ravindra, Theory of Seismic Head Waves (University of Toronto Press, Toronto, 1971), pp. 235-250.

19. R.A.Stephen and S.T.Bolmer, "The direct wave root in marine seismology", Bull. Seis. Soc. Am.,75,57-67(1985).

20. R.S. White and R.A. Stephen, "Compressional to shear wave conversion in oceanic crust," Geophys. J.R. astr. Soc., 63, 547-565 (1980).

21. P.K.P. Spudich and D.V. Helmberger, "Synthetic seismograms from model ocean bottoms," J. Geophys. Res. 84, 189-204 (1979).

22. R.A. Stephen, F. Pardo-Casas, and C.H. Cheng, "Finite difference synthetic acoustic logs", Geophysics 50, 1588-1609(1985).

23. M. Ottaviani, "Elastic wave propagation in two evenly-welded quarter-spaces", Bull. Seism. Soc. Am. 61, p.1119-1152(1971). 


\section{CHAPTER 2}

Seismic energy partitioning and scattering in laterally heterogeneous ocean crust 


\title{
Seismic energy partitioning and scattering in laterally heterogeneous ocean crust
}

\begin{abstract}
We present finite difference forward models of elastic wave propagation through laterally heterogeneous upper oceanic crust. The finite difference formulation is a 2-D solution to the elastic wave equation for heterogeneous media and implicitly calculates $\mathrm{P}$ and SV propagation, compressional to shear conversion, interference effects and interface phenomena. Random velocity perturbations with Gaussian and self-similar autocorrelation functions and different correlation lengths (a) are presented which show different characteristics of secondary scattering. Heterogeneities scatter primary energy into secondary body waves and secondary Stoneley waves along the water-solid interface. The presence of a water-solid interface in the models allows for the existence of secondary Stoneley waves which account for much of the seafloor 'noise' seen in the synthetic seismograms for the laterally heterogeneous models.
\end{abstract}

'Random' incoherent secondary scattering generally increases as $k a$ (wavenumber, $\mathrm{k}$, and correlation length, a) approaches one. Deterministic secondary scattering from larger heterogeneities is the dominant effect in the models as $k a$ increases above one. Secondary scattering also shows up as incoherence in the primary traces of the seismograms when compared to the laterally homogeneous case. Cross-correlation analysis of the initial P-diving wave arrival shows that, in general, the correlation between traces decreases as $k a$ approaches one. Also, because many different wave types exist for 
these marine models, the correlation between traces is range dependent, even for the laterally homogeneous case. 


\section{INTRODUCTION}

Marine seismic studies have been paramount in unravelling the gross structure of the oceanic crust. Various models of this structure have evolved and are constantly being refined as data collection, analysis, and modeling techniques become more effective. Currently under debate is the nature of the small-scale lateral heterogeneity of the crust as well as the depth dependence of the lateral heterogeneity. In the high frequency limit, that is, when the structure is much larger than the seismic wavelength $(\lambda)$, seismic energy propagates coherently through the crust and produces coherent seismogram arrivals. Information about large-scale features of crustal structure is obtained from the arrival times and slopes of these principal coherent arrivals. However, as we look for finer scale structure it becomes apparent that this simple analysis of seismograms is no longer adequate.

Aki (1982) defines scattering as "A process which generates incoherent signals by three-dimensional rough heterogeneities". As $k a$ approaches unity $(\mathrm{k}=2 \pi / \mathrm{l}$, $\mathrm{a}=$ heterogeneity length scale) the wave characteristics of the seismic energy come into the picture causing interference, diffractions, and in general much more complex patterns of propagation. Crustal heterogeneities act as scatterers of seismic energy and cause a degradation in the coherence of propagation through the crust. Thus, in order to examine the small scale heterogeneity of the crust it is the incoherence of the expected arrivals and the coda (or signal generated noise) which must be studied.

In general, most studies of scattering have been carried out to explain the amplitude attenuation and coda wave excitation of seismic energy from earthquake or teleseismic 
explosive sources (Aki, 1973,1982; Sato, 1982; McLaughlin, 1983, McLaughlin et.al.,1985, Frankel and Clayton, 1986). Large scale heterogeneities have the greatest influence on primary arrival amplitude attenuation while smaller scale heterogeneities seem to control coda wave frequency content and decay (Aki, 1973,1982). Most analytical work done to explain these results depends on the validity of the Born approximation which only considers the first interaction of energy with a scatterer (no multiple scattering) and that the scattering is weak with respect to the primary wave amplitude (Hudson and Heritage, 1981).

These previous studies are important foundations for this study but are not directly applicable for a number of reasons. Most estimates made of heterogeneities concern the entire lithosphere and parts of the upper mantle instead of just the crust (upper $5 \mathrm{~km}$ for oceanic crust). When the experiment is scaled to that of typical marine refraction work, the Born approximation is not necessarily valid (Hudson and Heritage, 1981). The case of scattering of vector (elastic) waves from a point source travelling through a medium with velocity gradients, topography, and random heterogeneities has little chance to be solved analytically. We wish to investigate heterogeneities with length scales less than the ranges of typical seismic refraction surveys (ranges from $5-50 \mathrm{~km}$ ).

An example of the influence of this size of lateral heterogeneities on the seismic signal is shown in figure 2.1. The data in figure 2.1 are from a borehole seismic experiment at the Deep Sea Drilling Project (DSDP) site 504B near the Costa Rica Rift (Stephen, 1987). This line of data was collected using a borehole receiver placed within the hole 42 meters into basement. Shots were fired in a circle of radius 6.0 kilometers and at azimuths from 0 to 360 degrees. Under the assumptions of lateral homogeneity and azimuthal isotropy, the arrivals in figure 2.1 should be perfectly coherent and have exactly the same arrival time. However, this is obviously not the case for the data shown here. In 
Figure 2.1. Borehole seismic data from the Deep Sea Drilling Project hole 504B near the Costa Rica Rift. These data were produced by placing a borehole receiver 42 meters into basement and firing a circle of surface shots (at azimuths from 0 to 360 degreees) at a range of 6.0 kilometers from the borehole. The effects of small scale lateral heterogeneity in the upper oceanic crust appear as coda (signal generated noise) and azimuth dependent amplitudes, travel times, and correlation. (figure from Stephen, 1987) 


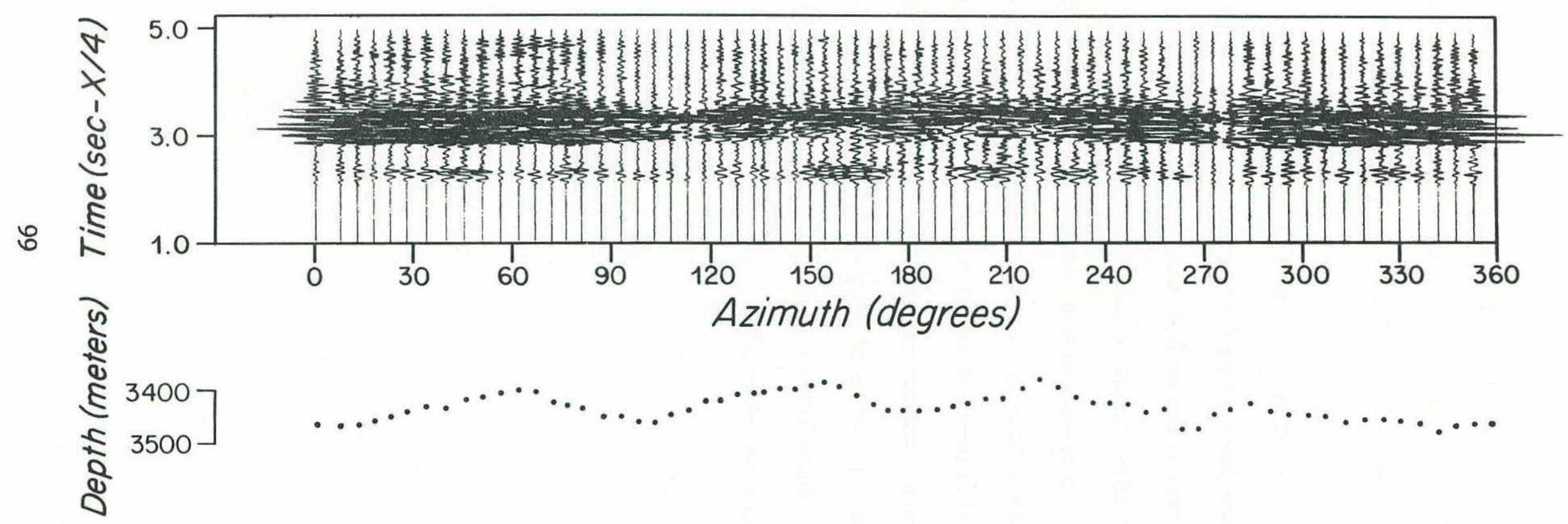

$6 \mathrm{~km}$ Circle Shot to $42 \mathrm{~m}$ into Basement/Vertical Component 
fact, there is a significant amount of signal generated noise, as well as large amplitude and travel time anomalies. It is not the intent of this study to reproduce the data seen in figure 2.1. but rather, we are interested in the types of seconday characteristics seen in these data.

In this study, we will use the finite difference method to solve the forward problem of seismic wave propagation through oceanic crust with random heterogeneity. Synthetic seismograms calculated by this method can be used to quantify the change in seismic traces due to the heterogeneities. An elastic finite difference formulation of the heterogeneous wave equation ( $\mathrm{P}$ and $\mathrm{S}$ velocity as well as density can be varied) allows us to create any realistic crustal model without the simplifying assumptions of most theoretical scattering analyses. Numerical Schlieren diagrams, or snapshots, of the energy propagating through the models show that a significant amount of energy is backscattered and converted by the heterogeneous crust. This scattered energy shows up as 'noise' on the synthetic seismogram traces. The effect of wave propagation through media with different length scales of heterogeneity are presented. 


\section{FINITE DIFFERENCE MODELING}

The finite difference mechod provides an excellent solution to the problem of wave propagation through random media. The finite difference scheme used in this paper is based on the scheme of Virieux (1986) and includes compressional and shear velocity variations and density variations. It was originally presented as a solution to the first order system in terms of particle velocities and stresses, but in order to reduce memory storage requirements we rewrote the formulation for the second order system in terms of particle displacements. The calculations are carried out in two dimensions for a line source in Cartesian coordinates. The equations solved are;

$$
\begin{aligned}
& \rho \frac{\delta^{2} u}{\delta t^{2}}=\frac{\delta \tau_{x x}}{\delta x}+\frac{\delta \tau_{x z}}{\delta z}, \\
& \rho \frac{\delta^{2} w}{\delta t^{2}}=\frac{\delta \tau_{x z}}{\delta x}+\frac{\delta \tau_{z z}}{\delta z}, \\
& \tau_{x x}=(\lambda+2 \mu) \frac{\delta u}{\delta x}+\lambda \frac{\delta w}{\delta z}, \\
& \tau_{z z}=(\lambda+2 \mu) \frac{\delta w}{\delta z}+\lambda \frac{\delta u}{\delta x}, \\
& \tau_{x z}=\mu\left(\frac{\delta u}{\delta z}+\frac{\delta w}{\delta x}\right),
\end{aligned}
$$

where $u, w$ are the horizontal and vertical displacements respectively, $\rho$ is density, $\tau$ is stress, and $\lambda, \mu$ are Lame's elastic parameters. Centered finite differences are used to approximate each derivative in space and time. Dependent and independent variables are 

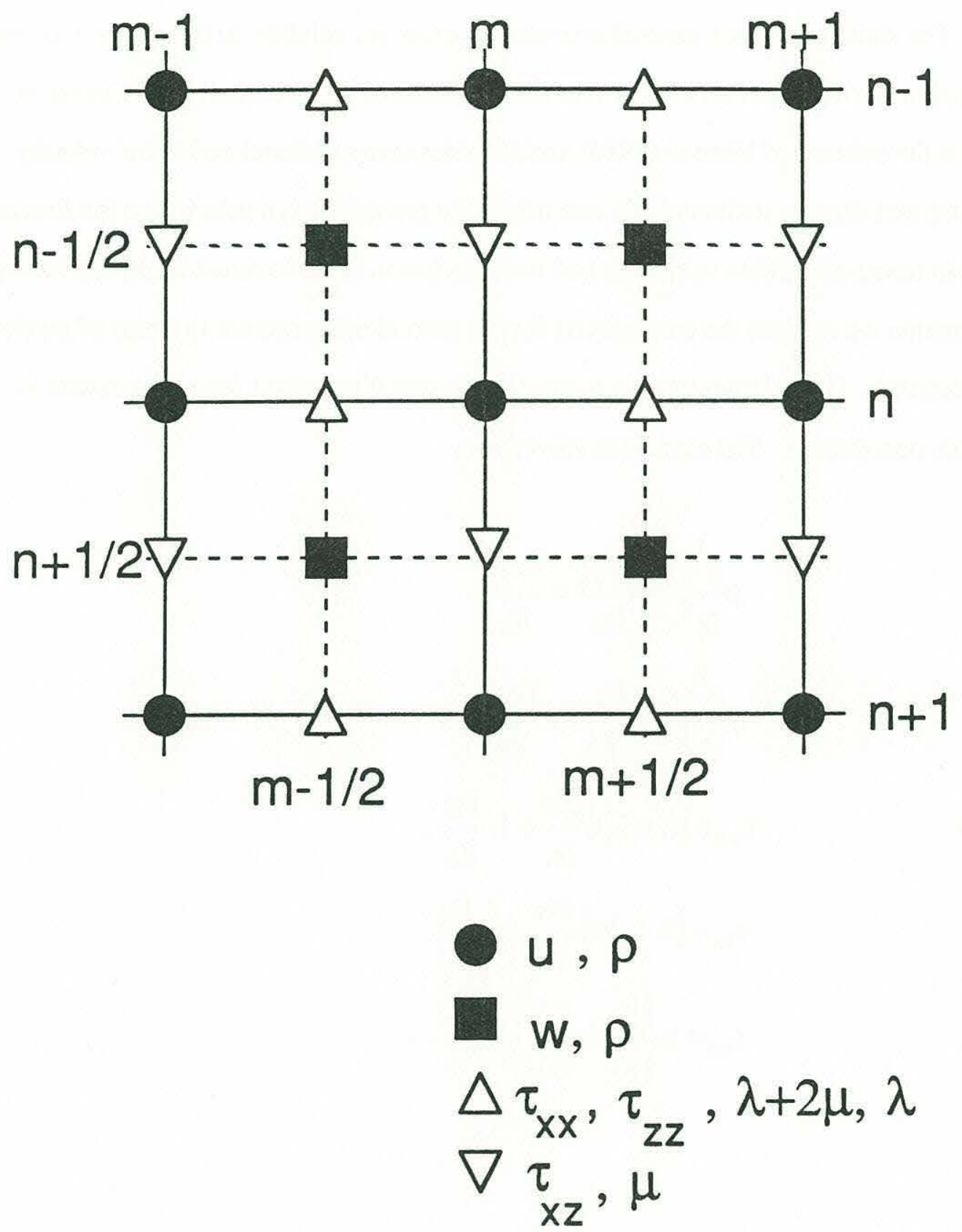

Figure 2.2. Location of dependent and independent variables on the finite difference grid. Horizontal and vertical displacements and stresses are defined at different points of the grid. (after Virieux, 1986) 
defined on the grid as shown in figure 2.2. This formulation implicitly calculates $\mathrm{P}$ and SV propagation ( $\mathrm{SH}$ is not present in the $\mathrm{x}-\mathrm{z}$ plane), compressional to shear conversion, interference effects and interface phenomena.

We prefer this modified Virieux scheme to the Nicoletis scheme used in previous studies (Nicoletis, 1981, Bhasavanija, 1983, Dougherty and Stephen, 1987 (chapter 1)) because is generates less parasitic noise, particularly in the water column. This noise, for the Nicoletis scheme is not large enough to contaminate time series results but it does spoil the appearance of snapshots and creates non-zero curl of the displacement field in the water column. These issues are discussed further in Stephen (1988). A complete comparison of this scheme with fourth order schemes (Alford et.al., 1974, Frankel and Clayton, 1986) has not been made by these authors but Fornberg (1987) has given some results which suggest that the fourth-order scheme has advantages. Since Fornberg (1987) did not investigate staggered grids, as used here, his results should be considered preliminary. Computer time and space requirements for the Virieux scheme were not prohibitive for these models and the accuracy of the seismograms for the Virieux method are excellent. A complete discusion of these issues is beyond the scope of this paper.

Grid layout and boundary conditions are given in figure 2.3. The grid is split into two homogeneous layers and a heterogeneous transition zone. Constant parameters in the homogeneous water $\left(\mathrm{V}_{\mathrm{p}}=1.5 \mathrm{~km} / \mathrm{s}, \mathrm{V}_{\mathrm{S}}=0.0, \rho=1.0\right)$ and deep crustal $\left(\mathrm{V}_{\mathrm{p}}=6.0, \mathrm{~V}_{\mathrm{S}}=3.46\right.$, $\rho=2.525$ ) layers allow a simplification of equation 1 and a saving in computation time. Compressional and shear velocities as well as density can be varied at each grid point within the transition zone.

The initial conditions are zero particle displacement and velocity everywhere on the grid. Boundary conditions for the models were chosen to minimize numerical aritifacts 
returning from the edges of the grid. The right hand side of the grid is an absorbing boundary based on paraxial approximations of the wave equation given by Clayton and Engquist (1977). This type of boundary was found to be unstable for the bottom boundary of the grid at large times. Therefore, reflections from the bottom boundary are suppressed by using a form of the telegraph equation combined with the homogeneous elastic wave equation (Levander, 1985, Cerjan et.al., 1985). Two attenuation terms are added to the wave equation which successively decrease wave amplitude in a region near the bottom boundary. The section of the grid which includes the telegraph formulation is shown by the stipled area in figure 2.3 .

The effect of the line source is introduced as a time dependent boundary condition on the top of the grid. A second grid is introduced along the top edge (see figure 2.3) to absorb upcoming energy and to prevent it from reflecting back into the region of interest as a false arrival. Up and downgoing wavefields are separated as in Alterman and Lowenthal (1972). The upgoing field is then absorbed at the top of the second grid using the Clayton and Engquist (1977) absorbing boundary condition.

As stated above, a source function is introduced at the top of the grid, along a layer $0.5 \mathrm{~km}$ (50 grid points at 10 meters per grid point) above the seafloor (see figure 2.3). The source waveform simulates a $10 \mathrm{~Hz}$ shot at the sea surface and is shown in figure 2.4. The shape of the pressure source wave is based on the second derivative of a Gaussian shape;

$$
\mathrm{p}(\mathrm{x}, \mathrm{z}, \mathrm{t})=\left(-\mathrm{A} /\left(4 \pi \alpha^{2} \mathrm{R}\right)\right) \mathrm{g}^{\prime \prime}(\mathrm{t}-\mathrm{R} / \alpha)
$$

where 


$$
\begin{aligned}
& g^{\prime \prime}(t)=4 \xi^{2}\left(3 T-2 \xi T^{3}\right) \exp \left(-\xi T^{2}\right) \\
& R=\left(x^{z}+z^{2}\right) 1 / 2 \quad, \quad T=t-t_{s}
\end{aligned}
$$

In equation $2, \mathrm{p}$ is the pressure field at time $t$ and coordinates $\mathrm{x}, \mathrm{z}, \mathrm{A}$ is a unit constant with dimensions (mass $\mathrm{x}$ length 2 / time), $\alpha$ is the compressional velocity of water, $\mathrm{g}$ is a Gaussian curve, $t_{s}$ is a time shift parameter, and $\xi$ is a pulse width parameter for frequency scaling (Stephen et.al., 1985, Kelly et.al., 1979). A value of 657 for $\xi$ was used which produces a signal with peak frequency of $10 \mathrm{~Hz}$ and an upper half power frequency of 13.5 Hz. The time increment used was 0.001 seconds.

A number of different output formats are available from the finite difference method. In fact, one of the advantages of the method is the ability to save particle displacements for any number of points on the grid and for all times. A particularly informative way to view wave propagation through the models is by using numerical Schlieren diagrams. These 'snapshots' of the wave field travelling through the grid are produced by saving the value of horizontal and vertical displacement at each point in the grid for a given time step. The quantities plotted in the Schlieren diagrams are related to compressional and shear energies and are calculated by using the spatial divergence and curl operators on the displacements. Morse and Feshbach (1953) define compressional $\left(E_{C}\right)$ and shear $\left(E_{S}\right)$ energy as;

$$
\begin{aligned}
& E_{\mathrm{c}}=(\lambda+2 \mu)(\nabla \bullet \mathbf{u})^{2} \\
& \mathrm{E}_{\mathrm{S}}=\mu(-\nabla \mathrm{xu})^{2}
\end{aligned}
$$


Figure 2.3. Layout of the finite difference grid for the models presented. The source is introduced along a layer 50 grid points above the water-solid interface. Homogeneous layers of water (top of grid) and crust (bottom) are solved using a formulation of the homogeneous wave equation. The heterogeneous transition zone contains all lateral and vertical velocity (both compressional and shear) and density variations. Energy is attenuated by using the telegraph equation in the stipled area at the bottom of the grid. 


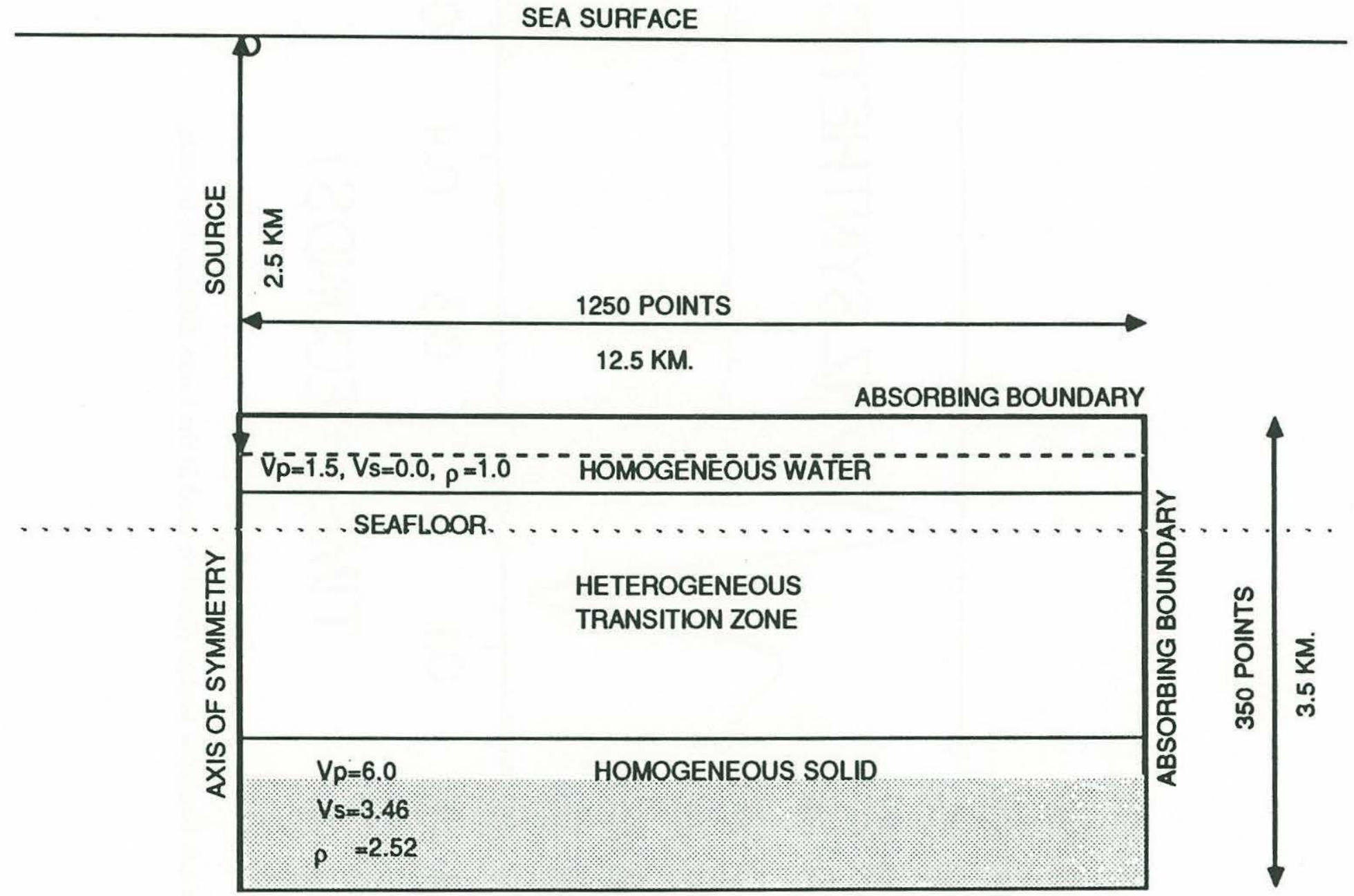




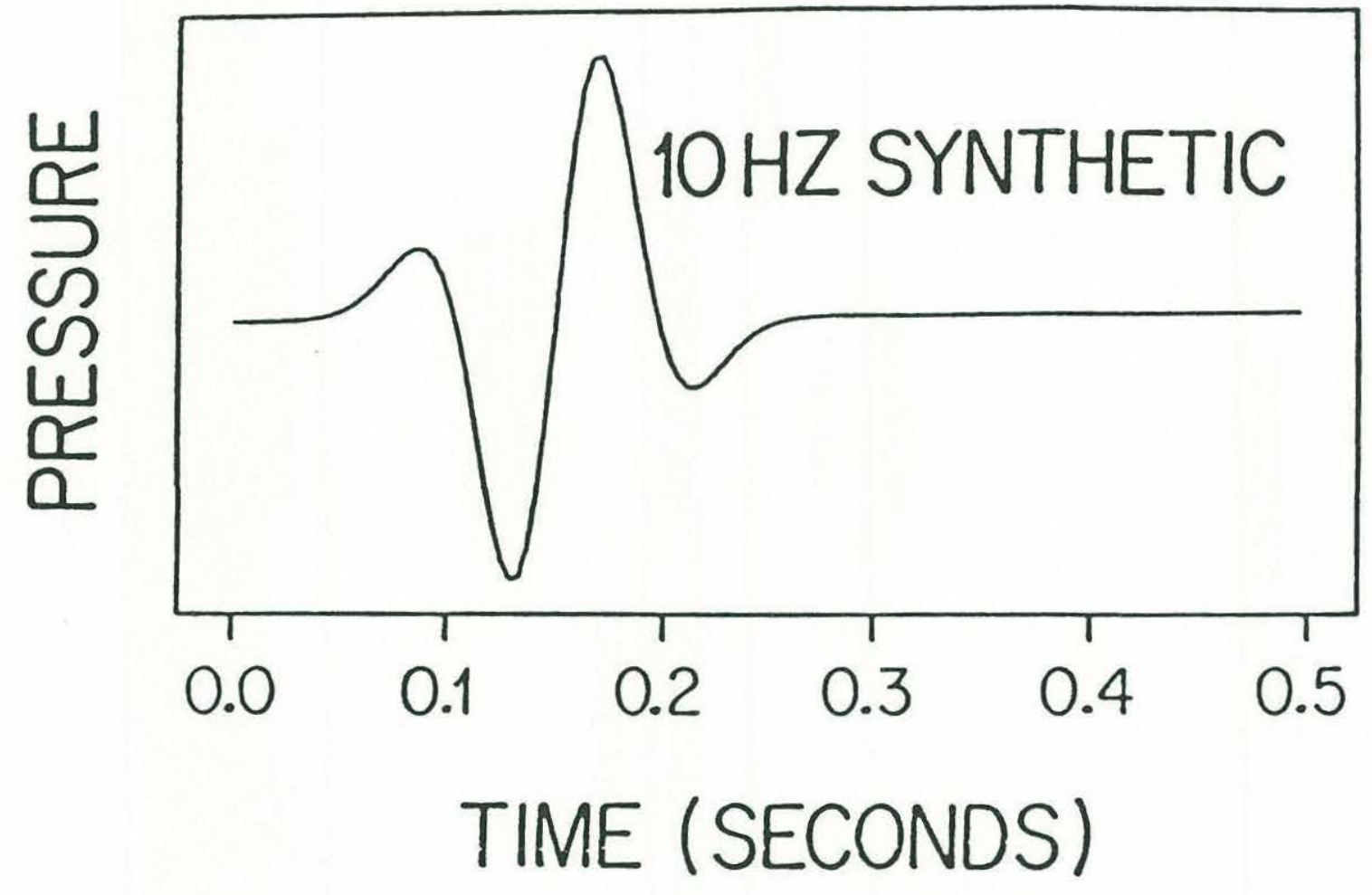

Figure 2.4. Pressure source function used in the finite difference models. 
In order to preserve the divergence and curl sign information while showing relative compressional and shear energy amplitudes, we plot the following normalized quantities in the numerical Schlieren diagrams;

$$
\begin{aligned}
& \text { Norm } P=\operatorname{sign}(\nabla \bullet u) *\left(E_{c}\right)^{1 / 2} \\
& \text { Norm } S=\operatorname{sign}(\nabla x u) *(E s) 1 / 2
\end{aligned}
$$

Synthetic seismograms are produced by saving displacements and/or pressure at any point on the grid for a series of timesteps. Time series of horizontal and vertical displacements, as well as pressure were produced for a row of 'receivers' 20 meters above the water-solid interface and a row 30 meters below the interface. This was done to compare the pressure signal at a water column hydrophone to displacements from a buried geophone. Receivers were placed every four grid points (equivalent to 40 meter spacing) for each row and values were saved for every fourth timestep (.004 second sampling interval).

\section{SCATTERING MODELS}

A number of models were run with different length scales of heterogeneity and spatial filter types to examine the effect on the seismograms. A control model, FLAT, was first run to demonstrate wave propagation through an upper oceanic crust with no lateral heterogeneity. Figure 2.5 presents the velocity-depth function used for FLAT. The velocity-depth function is based on results of Purdy (1982) and Ewing and Purdy (1982) for young ocean crust studied in the Rivera Ocean Seismic Experiment. Dougherty and Stephen (1987, chapter 1) used a similar V-Z function to model deterministic scattering 
from seafloor features. All of the models assume a Poisson's ratio of 0.25 and the density ( $r$ ) is calculated from P-wave velocity by the following relation (Nafe and Drake, 1957);

$$
\rho(x, z)=.252+(.3788) * V_{p}(x, z)
$$

Lateral heterogeneity was created by adding a series of normally distributed random velocity variations to the V-Z function of FLAT. Each grid point in the crustal section of the heterogeneous transition zone has a random component added to it. The random velocity variations have zero mean so that the average velocity for any given layer is approximately equal to the velocity of that layer in the laterally homogeneous case. The heterogeneity standard deviation is $+/-10$ percent at the water solid interface and decreases down to zero at a depth of $1.3 \mathrm{~km}$. (130 grid points ) below the interface. The decrease in heterogeneity with depth is meant to simulate the sealing of cracks and fissures with depth in the upper oceanic crust.

After adding random velocity variations to each grid point in the transition zone, the entire velocity field was spatially filtered to create different heterogeneity correlation lengths. A filter which produced a Gaussian autocorrelation function was used for most of the models in this study. Spatial correlation lengths of 50,100, and 200 meters where used for models GAUSS50, GAUSS100, and GAUSS200 respectively. One model with a self-similar autocorrelation function and a correlation length of 200 meters is also presented (SELFSIM200). The self-similar distribution is based on the fractal geometry of Mandelbrot (1977) and contains uniform fluctuations over a range of length scales. Frankel and Clayton (1986) describe these filter correlation functions in terms of their definitions and fluctuation power spectra. 
Figure 2.6 shows the velocity fields of the five heterogeneous models. GAUSS10 has just the original unfiltered random number sequence added to the gradients of FLAT. Although this would be an uncorrelated sequence for the continuous case, the discrete 10 meter grid spacing of the finite difference formulation imposes a correlation length of 10 meters on the velocity field of GAUSS10. GAUSS50, GAUSS100, and GAUSS200 are based on the same initial random number sequence but are filtered with different correlation lengths of a Gaussian filter. SELFSIM200 has a self-similar aoutocorrelation function with a correlation length of 200 meters.

\section{Laterally homogeneous model}

Before examining propagation through the random models, it is useful to review energy propagation and partitioning for this seafloor geometry with the laterally homogeneous case (FLAT). Unlike propagation of plane or spherical waves through a halfspace, simulations of marine refraction experiments contain a water-solid interface underlain by a region of velocity gradient. This drastic change in elastic parameters results in a number of interesting partitioning, conversion, and interface effects. Figure 2.7 is a series of numerical Schlieren diagrams which show the propagation of normalized compressional and shear energy across the FLAT velocity field. These 'snapshots' are shown for 0.4 second intervals starting at 2.0 seconds after the shot. The top of each frame corresponds to a depth of $2.5 \mathrm{~km}$ below the sea surface with the sea floor at $3.0 \mathrm{~km}$. depth. The bottom of the grid corresponds to a total depth of $5.5 \mathrm{~km}$. $(2.5 \mathrm{~km}$ below the water-solid interface). 
Figure 2.5. Compressional velocity field for the laterally homogeneous model FLAT. Pvelocity (a) at the water-solid interface is $3.2 \mathrm{~km} / \mathrm{sec}$ and increases to $6.0 \mathrm{~km} / \mathrm{sec}$ at a depth of $1.3 \mathrm{~km}$ below the interface (total depth $4.3 \mathrm{~km}$ ). A steep gradient of $4.0 \mathrm{sec}^{-1}$ is present from the interface to $0.4 \mathrm{~km}$ below the interface, with a second less steep gradient of 1.33 $\mathrm{sec}^{-1}$ from 0.4 to $1.3 \mathrm{~km}$. below the interface. It is assumed that the crust is a Poisson's solid so that the shear velocity, $\beta=\alpha /(3)^{1 / 2}$. 


\section{FLAT}
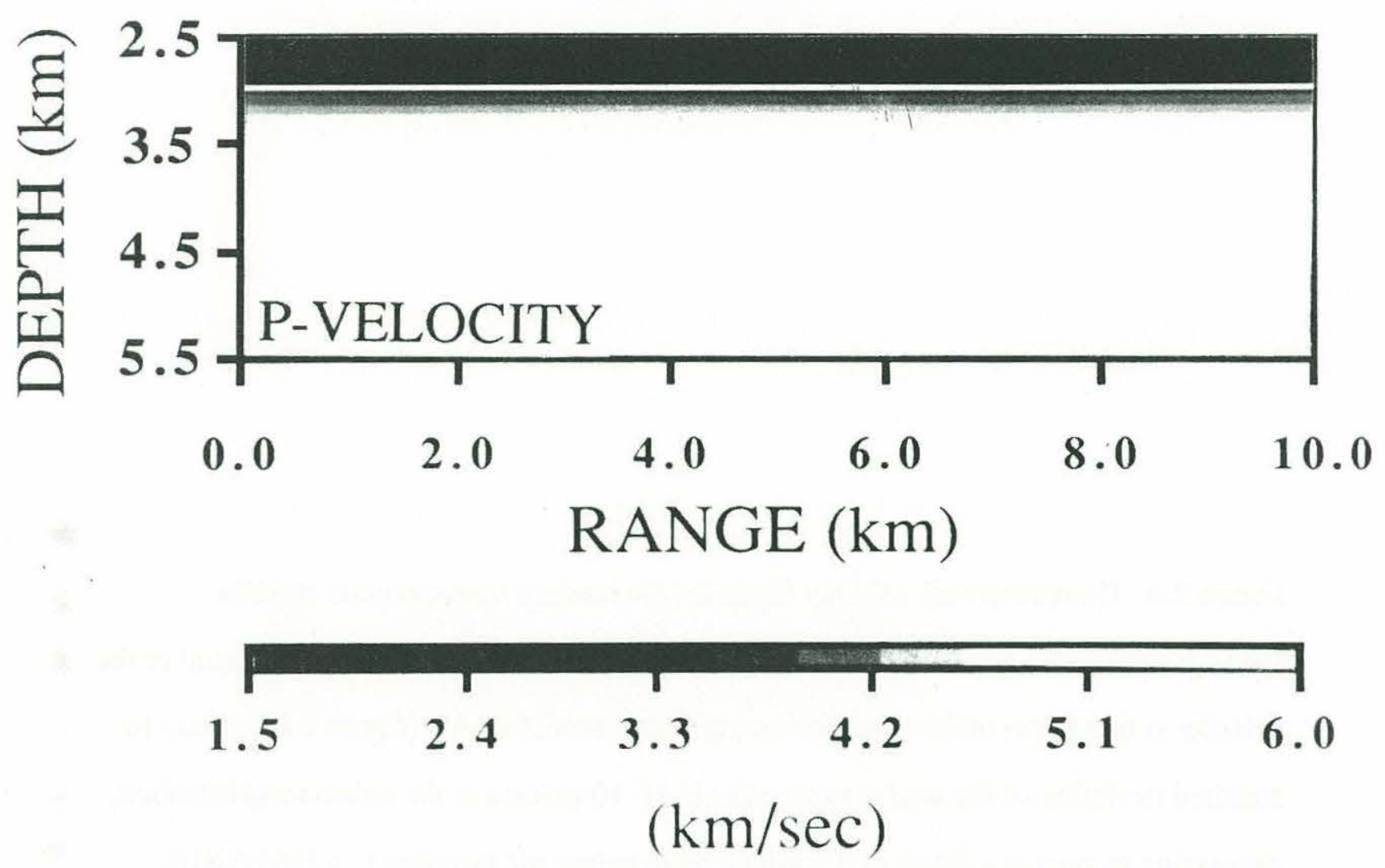
Figure 2.6. Compressional velocity fields for the random heterogeneity models GAUSS10-GAUSS200. In each case, the average velocity for each depth is equal to the velocity at that depth in the laterally homogeneous model FLAT (figure 2.5). Also, the standard deviation of the random velocities is $+/ 10$ percent at the water-solid interface, decreasing to zero at a depth of $4.3 \mathrm{~km}$ (1.3 km below the interface). a.GAUSS10, Gaussian random perturbations applied to each grid point, since no spatial filtering is done, the effective correlation length for GAUSS 10 is 10 meters (equal to the grid spacing). b. GAUSS50, velocity field of GAUSS10 filtered with a 2-D Gaussian spatial filter, correlation length equal to 50 meters. c. GAUSS100, Gaussian spatial filter, correlation length of 100 meters. d. GAUSS200, Gaussian spatial filter, correlation length 200 meters. e. SELFSIM200, self-similar spatial filter, correlation length 200 meters. Note in the latter case, the presence of smaller scale roughness superimposed on the larger features of the filtered field. 

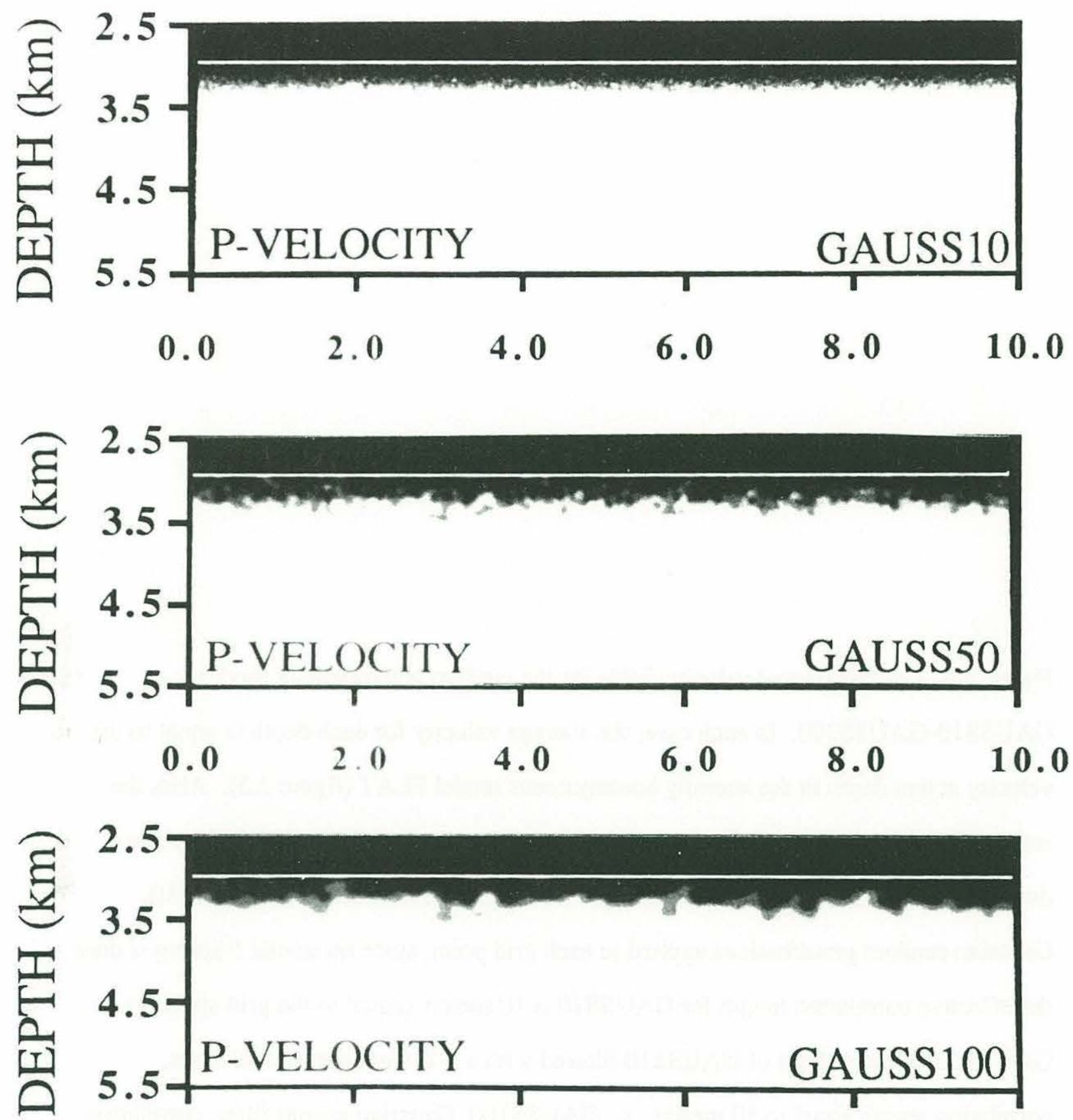
0.0
2.0
4.0
6.0
8.0
10.0
RANGE (km)

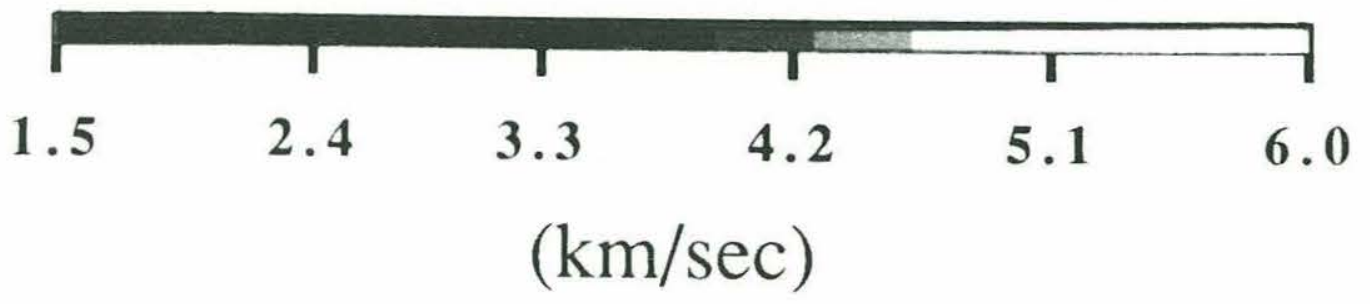


Figure 2.6. Compressional velocity fields for the random heterogeneity models GAUSS10-GAUSS200. In each case, the average velocity for each depth is equal to the velocity at that depth in the laterally homogeneous model FLAT (figure 2.5). Also, the standard deviation of the random velocities is $+/-10$ percent at the water-solid interface, decreasing to zero at a depth of $4.3 \mathrm{~km}$ (1.3 km below the interface). a.GAUSS10, Gaussian random perturbations applied to each grid point, since no spatial filtering is done, the effective correlation length for GAUSS10 is 10 meters (equal to the grid spacing). b. GAUSS50, velocity field of GAUSS10 filtered with a 2-D Gaussian spatial filter, correlation length equal to 50 meters. c. GAUSS100, Gaussian spatial filter, correlation length of 100 meters. d. GAUSS200, Gaussian spatial filter, correlation length 200 meters. e. SELFSIM200, self-similar spatial filter, correlation length 200 meters. Note in the latter case, the presence of smaller scale roughness superimposed on the larger features of the filtered field. 


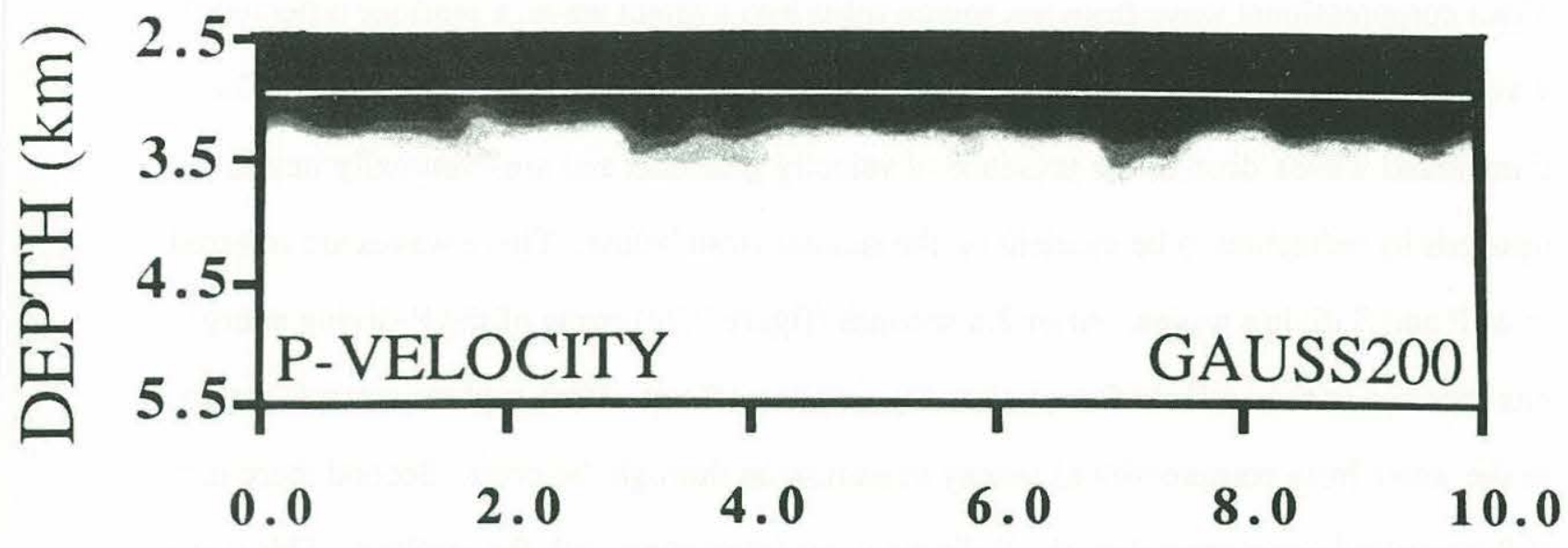

D

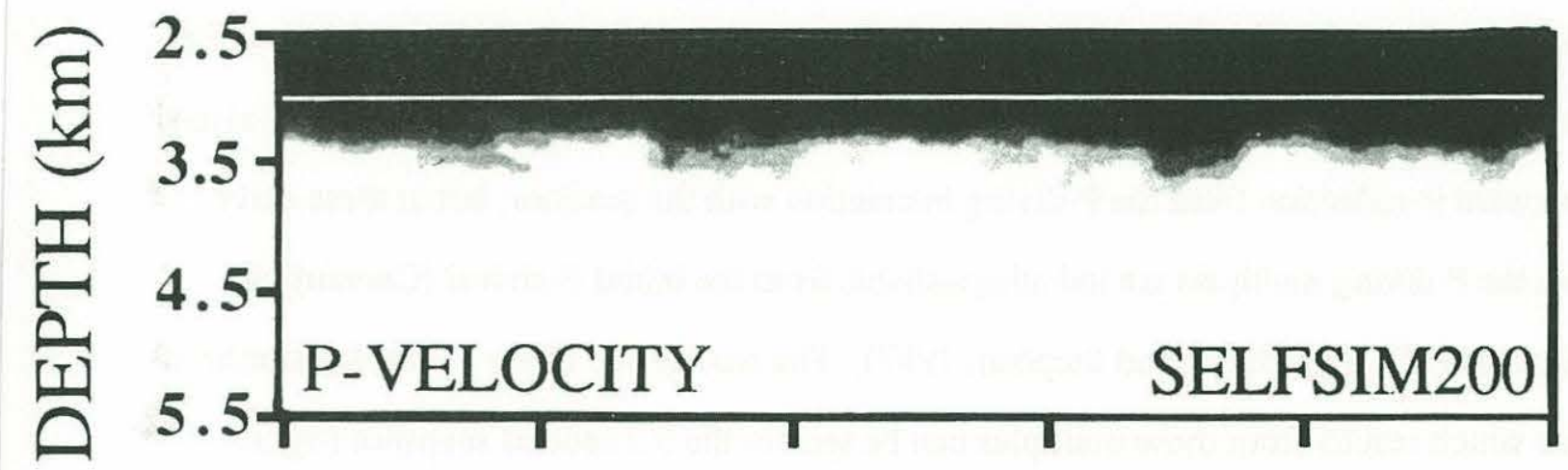
0. 0
2.0
4.0
6.0
8.0
10.0
RANGE (km)

E

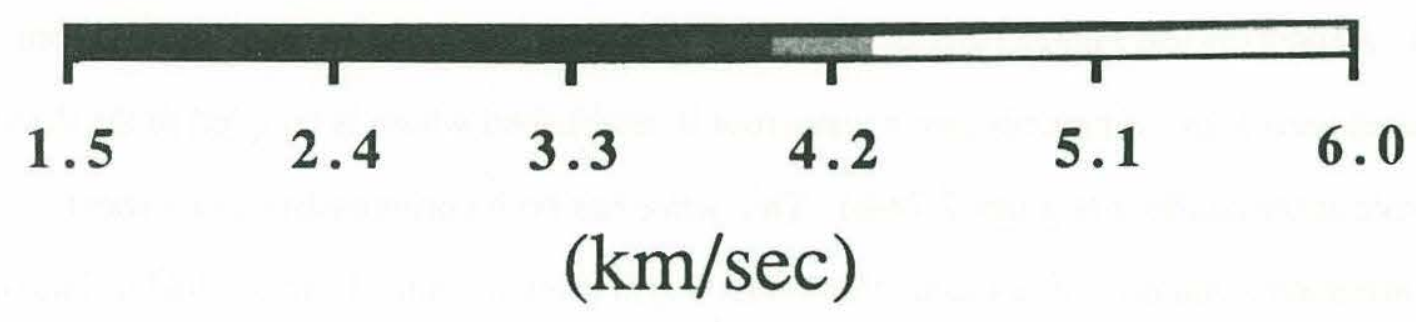


FLAT represents a section of 'typical' young ocean crust with no lateral heterogeneity. With a seafloor at $3 \mathrm{~km}$ depth, it takes 2 seconds for the source to reach the seafloor, as can be seen in figure 2.7a. After incidence on the water-solid interface, the direct compressional wave from the source splits into a direct wave, a seafloor reflected Pwave, a transmitted P-wave, and a converted transmitted shear wave (figure $2.7 \mathrm{~b}$ ). The transmitted waves 'dive' in the presence of velocity gradients and are eventually turned upwards by refraction to be incident on the seafoor from below. These waves are referred to as $\mathrm{P}$ and $\mathrm{S}$ diving waves. After 2.8 seconds (figure 2.7c) some of the P-diving energy has returned to the seafloor from below causing two effects. First, a plane wave is set up in the water from compressional energy transmission through the crust. Second there is a P-S converted wave caused by the P-diving wave interaction with the seafloor. This wave is the $\mathrm{P}_{1} \mathrm{P}_{2} \mathrm{~S}_{2}$ wave (Brekhovskikh, 1960) and is seen coupled to the P-diving wave at the water-solid interface and tangential to the S-diving wave at depth. Of course, there is also a downward P-reflection from the P-diving interaction with the seafloor, but at these early times the P-diving multiples are indistinguishable from the initial P-arrival (Cerveny and Ravindra, 1971, Dougherty and Stephen, 1987). The emergence of the P-interference head wave which results from these multiples can be seen in the 3.2 second snapshot (figure $2.7 d)$.

Eventually, the S-diving wave is turned back to be incident on the seafloor from below and shows the same general characteristics of the P-diving wave (see figures $2.7 \mathrm{~d}$ h). At post critical ranges (that is, after the P and S-diving waves have separated from the direct wave), an evanescent direct wave root is established which is coupled to the direct wave at the seafloor (figures $2.7 \mathrm{e}-\mathrm{h}$ ). This wave has both compressional and shear components and dies off exponentially with depth (Stephen and Bolmer, 1985). The only other major wave to develop is the pseudo-Rayleigh wave which appears in the 4.4 and 4.8 second snapshots (figures $2.7 \mathrm{~g}-\mathrm{h}$ ). 
Synthetic seismograms from model FLAT are shown in figure 2.8 for hydrophones and buried receivers along the seafloor. The Schlieren diagrams for FLAT reveal that the interactions between the different waves present is quite complex even for this laterally homogeneous case. However, it is important to note that all of the waves appear as coherent arrivals in the seismograms. Deterministic scattering from laterally homogeneous crust is complex but predictable. This is evidenced by apparently strong correlation between seismic traces in figure 2.8 .

\section{Random models}

The object of this study is to demonstrate the degradation in the seismic signal which is caused by wave scattering from random velocity variations in the upper oceanic crust. A series of models with Gaussian velocity autocorrelation functions were run to demonstrate the effect on the seismic signal. The Gaussian autocorrelation function was chosen for most of the models because it is a particularly well studied problem (Chernov, 1960). One model with a self-similar (fractal) distribution of velocity fluctuations is also presented.

Model GAUSS50, as with all of the following models, contains a $+/-10$ percent random velocity fluctuation at the water-solid interface. The fluctuation decreases to zero at a depth of $1.3 \mathrm{~km}$. below the seafloor (see figure 2.6). The velocity field of GAUSS50 has a Gaussian autocorrelation function with a characteristic correlation length of 50 meters. Since a predominantly $10 \mathrm{~Hz}$ source is used in these models, $k a$ is approximately equal to one at the seafloor. Wavefront snapshots for model GAUSS50 are presented in figure 2.9. Synthetic seismograms for water column and buried receivers are shown in 
Figure 2.7. Numerical Schlieren diagrams of normalized compressional and shear (top and bottom, respectively) energy for the laterally homogeneous model FLAT. The frames correspond to the ranges $0-10 \mathrm{~km}$. in the model geometry shown in figure 2.3. After a simulated surface shot, the direct wave is just reaching the seafloor after 2.0 seconds (fig. 2.7a) and partitions into direct, reflected, transmitted $\mathrm{P}$ and transmitted $\mathrm{S}$ waves after interaction with the seafloor (fig. 2.7b). The $\mathrm{P}_{1} \mathrm{P}_{2} \mathrm{~S}_{2}$ arrival is seen after 2.8 seconds (fig. 2.7c) and the interference P-wave is starting to be distinct after 3.2 seconds (fig. $2.7 \mathrm{~d}$ ). P and $\mathrm{S}$ components of the direct wave root show up as evanescent waves coupled to the direct wave after the $\mathrm{P}$ and $\mathrm{S}$ diving waves have separated from the direct wave (fig. $2.7 \mathrm{f}$ h). The only other major wave to occur is the pseudo-Rayleigh wave seen in figure $2.7 \mathrm{~g}$ h. 


\section{FLAT}
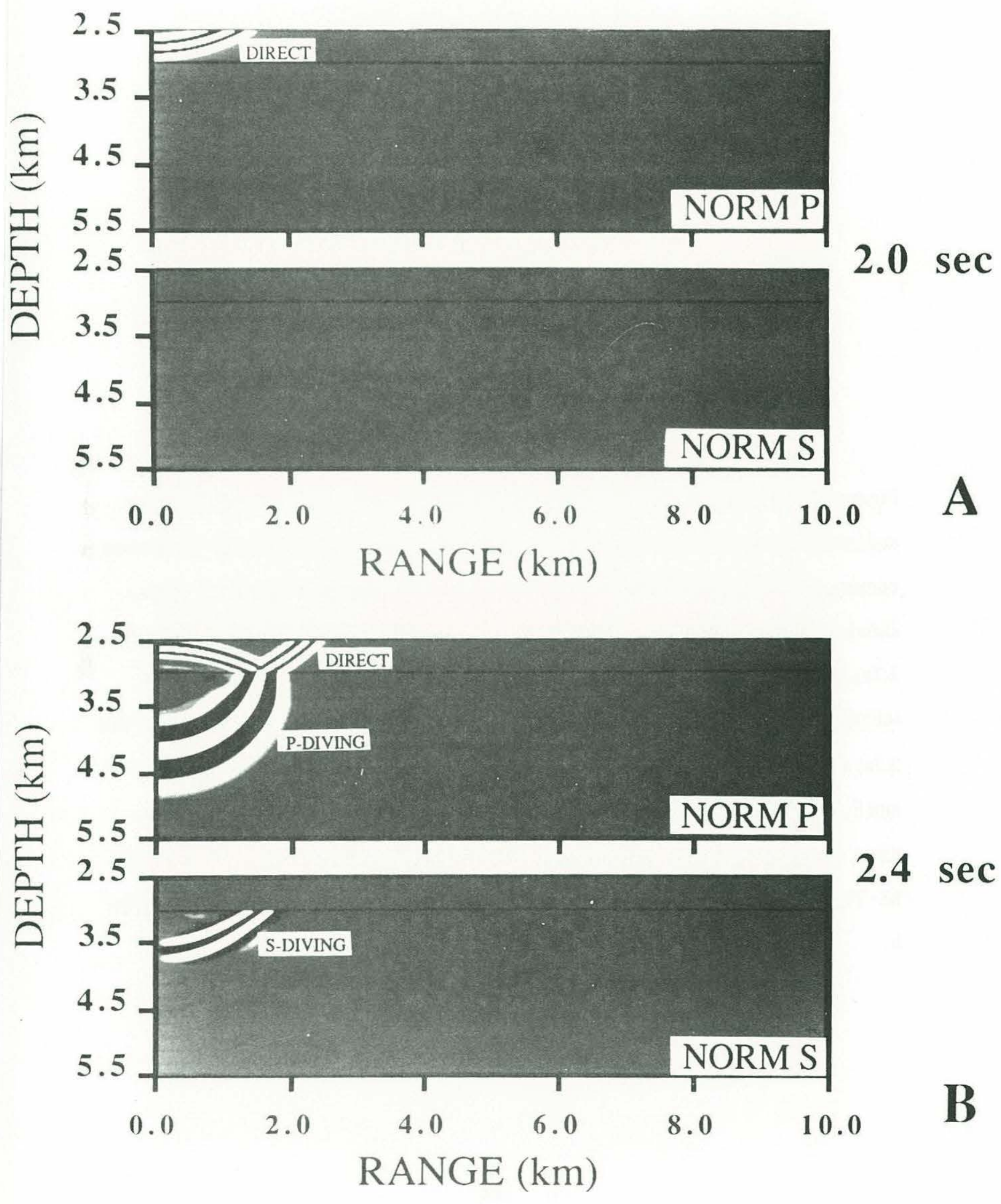
Figure 2.7. Numerical Schlieren diagrams of normalized compressional and shear (top and bottom, respectively) energy for the laterally homogeneous model FLAT. The frames correspond to the ranges $0-10 \mathrm{~km}$. in the model geometry shown in figure 2.3. After a simulated surface shot, the direct wave is just reaching the seafloor after 2.0 seconds (fig. 2.7a) and partitions into direct, reflected, transmitted $\mathrm{P}$ and transmitted $\mathrm{S}$ waves after interaction with the seafloor (fig. 2.7b). The $\mathrm{P}_{1} \mathrm{P}_{2} \mathrm{~S}_{2}$ arrival is seen after 2.8 seconds (fig. 2.7c) and the interference P-wave is starting to be distinct after 3.2 seconds (fig. $2.7 \mathrm{~d}$ ). P and $\mathrm{S}$ components of the direct wave root show up as evanescent waves coupled to the direct wave after the $\mathrm{P}$ and $\mathrm{S}$ diving waves have separated from the direct wave (fig. $2.7 \mathrm{f}$ h). The only other major wave to occur is the pseudo-Rayleigh wave seen in figure $2.7 \mathrm{~g}$ h. 


\section{FLAT}
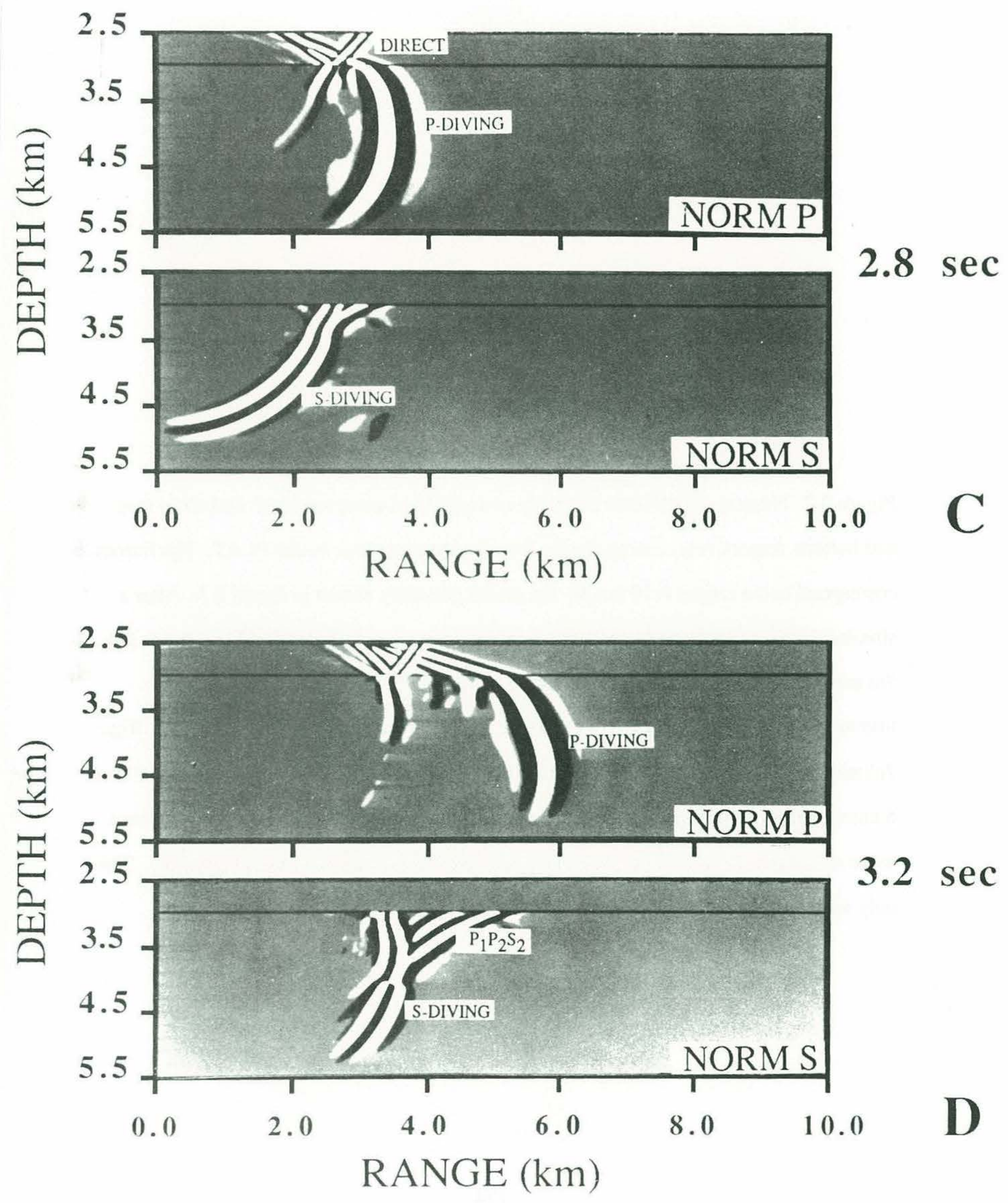
Figure 2.7. Numerical Schlieren diagrams of normalized compressional and shear (top and bottom, respectively) energy for the laterally homogeneous model FLAT. The frames correspond to the ranges $0-10 \mathrm{~km}$. in the model geometry shown in figure 2.3. After a simulated surface shot, the direct wave is just reaching the seafloor after 2.0 seconds (fig. 7a) and partitions into direct, reflected, transmitted $\mathrm{P}$ and transmitted $\mathrm{S}$ waves after interaction with the seafloor (fig. 7b). The $\mathrm{P}_{1} \mathrm{P}_{2} \mathrm{~S}_{2}$ arrival is seen after 2.8 seconds (fig. 7c) and the interference P-wave is starting to be distinct after 3.2 seconds (fig. 7d). P and $\mathrm{S}$ components of the direct wave root show up as evanescent waves coupled to the direct wave after the P and S diving waves have separated from the direct wave (fig. $7 \mathrm{f}-\mathrm{h}$ ). The only other major wave to occur is the pseudo-Rayleigh wave seen in figure $7 \mathrm{~g}$-h. 


\section{FLAT}
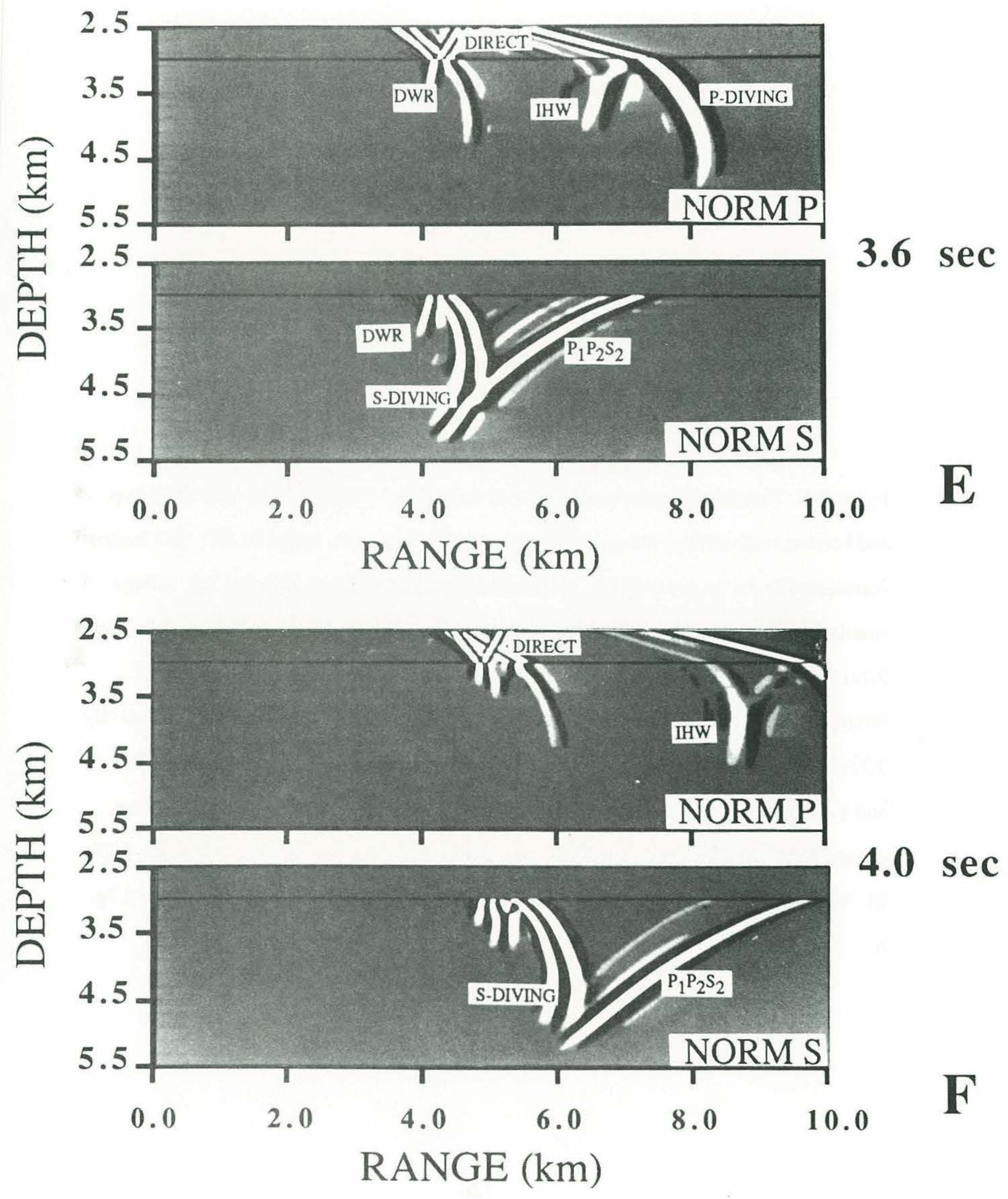
Figure 2.7. Numerical Schlieren diagrams of normalized compressional and shear (top and bottom, respectively) energy for the laterally homogeneous model FLAT. The frames correspond to the ranges $0-10 \mathrm{~km}$. in the model geometry shown in figure 2.3 . After a simulated surface shot, the direct wave is just reaching the seafloor after 2.0 seconds (fig. 2.7a) and partitions into direct, reflected, transmitted $\mathrm{P}$ and transmitted $\mathrm{S}$ waves after interaction with the seafloor (fig. 2.7b). The $\mathrm{P}_{1} \mathrm{P}_{2} \mathrm{~S}_{2}$ arrival is seen after 2.8 seconds (fig. 2.7c) and the interference P-wave is starting to be distinct after 3.2 seconds (fig. $2.7 \mathrm{~d}$ ). P and $\mathrm{S}$ components of the direct wave root show up as evanescent waves coupled to the direct wave after the P and S diving waves have separated from the direct wave (fig. 2.7fh). The only other major wave to occur is the pseudo-Rayleigh wave seen in figure $2.7 \mathrm{~g}$ h. 
FLAT
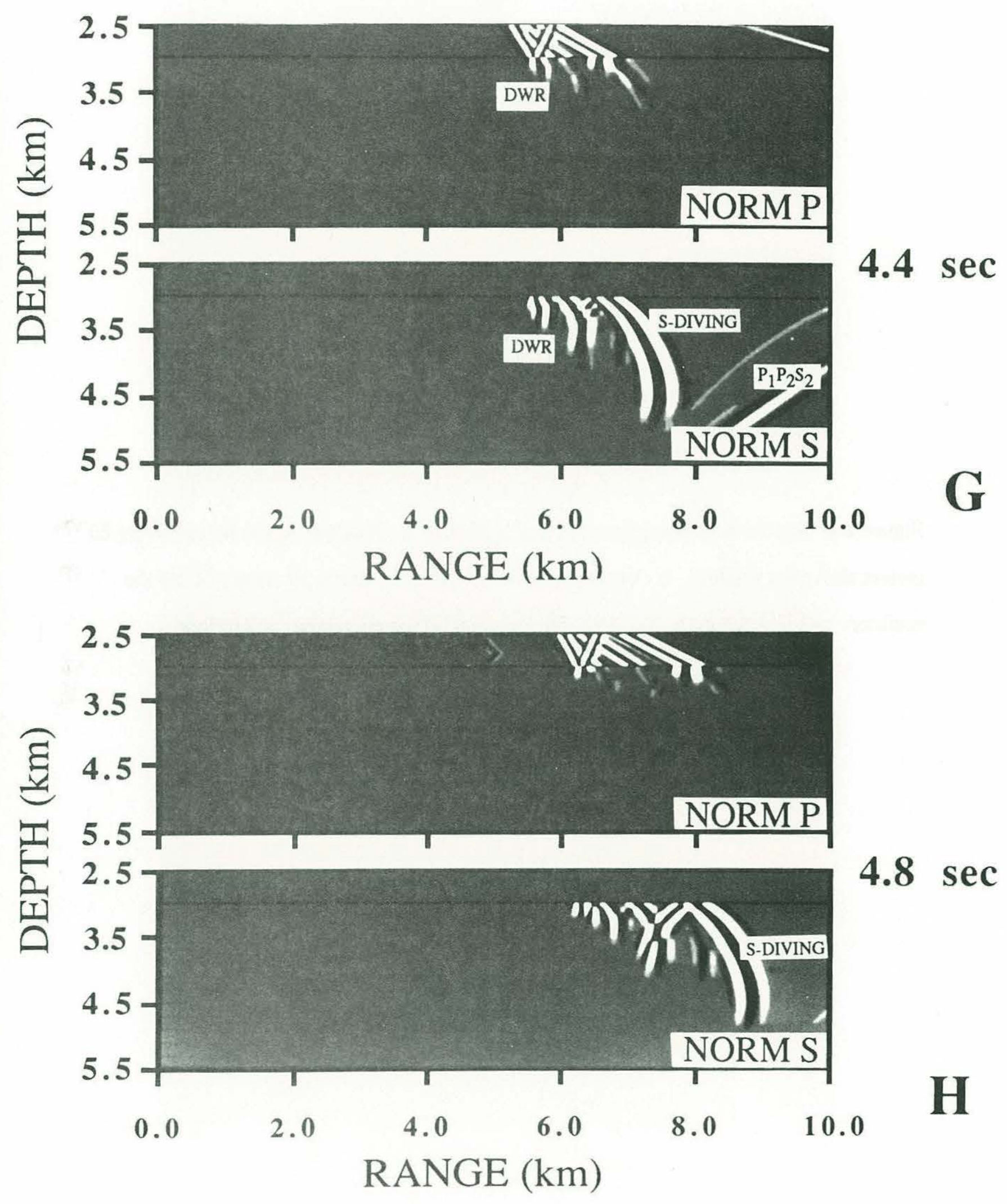
Figure 2.8. Synthetic seismograms for model FLAT. a. Pressure signal for receivers 20 meters above the seafloor. b. Vertical displacement for receivers 30 meters below the seafloor. c. Horizontal displacement for receivers 30 meters below the seafloor. 


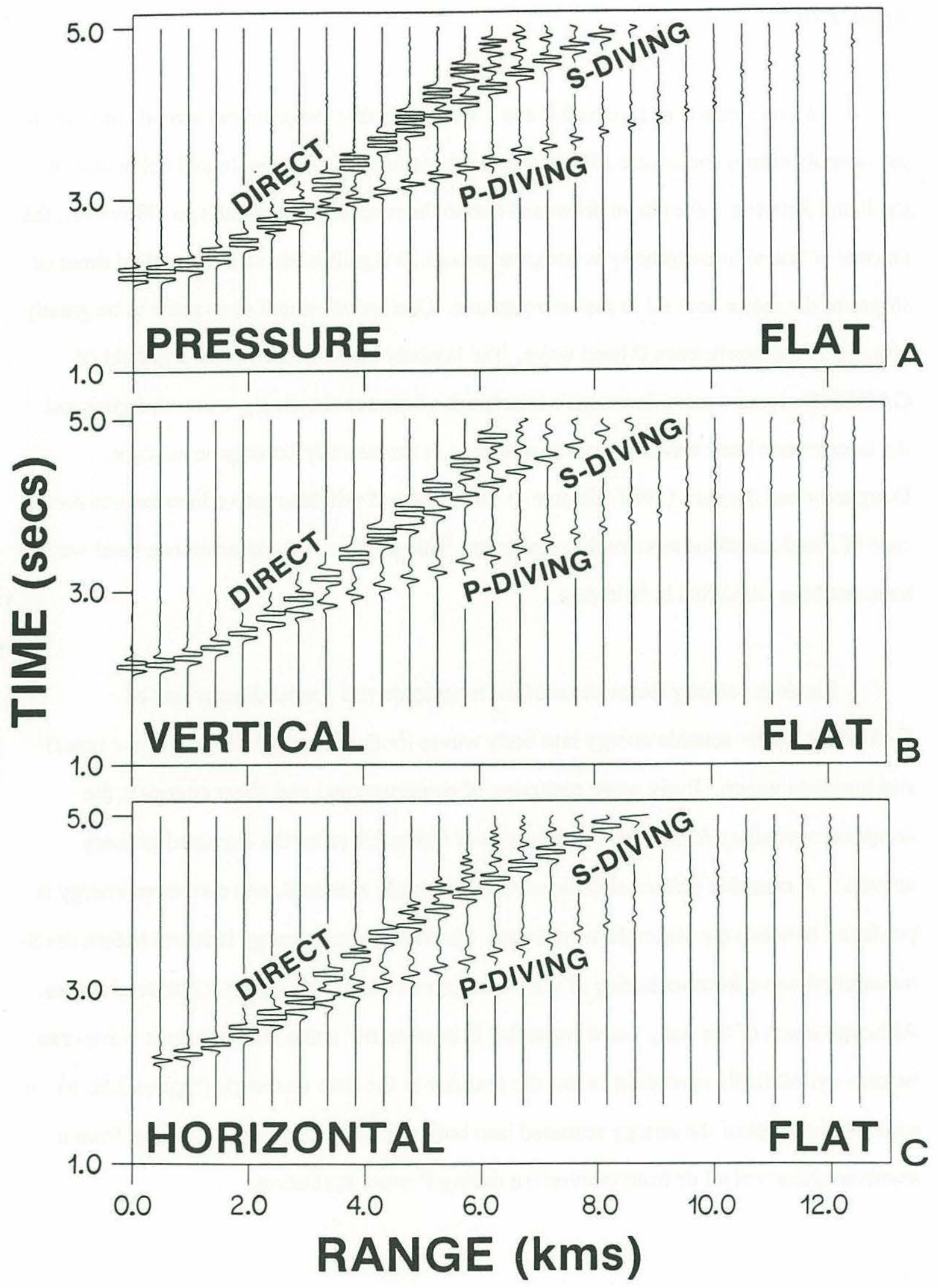


figure 2.10 .

An examination of figures 2.9 and 2.10 reveals that the principal arrivals present in the laterally homogenous case still dominate the results. The amplitude and coherence of the $\mathrm{P}$ and $\mathrm{S}$ diving waves have decreased due to the random heterogeneities. However, the amount of lateral heterogeneity is not great enough to significantly alter the arrival times or slopes of the major arrivals in the seismograms. One arrival which does seem to be greatly affected is the interference P-head wave. The laterally heterogeneous velocity field of GAUSS50 causes a more destructive interference between the diving wave multiples and the interference head waves are not as strong as in the laterally homogeneous case. Dougherty and Stephen (1987, chapter 1) also observed this destructive interference for the case of simple seafloor topographic scatterers. This explains why interference head waves have not been identified in field data.

Random velocity fluctuations of the magnitude and spatial dimensions of GAUSS50 scatter seismic energy into body waves (both compressional and shear types) and interface waves. Body wave scattering of compressional and shear energy is the dominant secondary feature in the snapshots of figure 2.9 (after the expected primary arrivals). A complex pattern of backscattered, multiply scattered, and converted energy is produced between the principle wavefronts. Converted shear energy is found before the Stransmitted wave from scattering of the P-diving wave into both $\mathrm{P}$ and S scattered waves. Although much of the body wave scattering is incoherent, some faint coherent waves can be seen cylindrically spreading below the seafloor in the later timesteps (figures $2.9 \mathrm{c}-\mathrm{h}$ ). It appears that most of the energy scattered into body waves is shear energy, either from a scattered shear arrival or from conversion during P-wave scattering. 
A great deal of energy is scattered by the lateral velocity fluctuations back into the water column. The backscattered body waves appear as plane waves in the water column and show up in the time series as relatively incoherent 'noise'. Upon closer examination of the time series, coherent sections of scattered body waves can be observed. Most of the coherent scattered body waves are shear arrivals and travel with a speed of approximately $2.8 \mathrm{~km} / \mathrm{sec}$. Figure 2.11 is a plot of two sections of the time series from receivers with a spacing of 40 meters (instead of 480 meter spacing in figures 2.8 and 2.10). Small sections of coherent S-phases are present which are not obviously coherent when observed with a larger seismogram spacing.

Another effect of secondary scattering of the primary arrivals is the generation of secondary Stoneley waves along the water-solid interface. These waves do not appear in the laterally homogeneous case because the only source is located too far from the interface. However, in the laterally heterogeneous models, velocity fluctuations of a significant magnitude and spatial dimension can act as secondary sources of seismic energy when encountered by primary source energy. If these secondary sources are in close enough proximity to the water-solid interface, secondary Stoneley waves can be generated.

Secondary Stoneley waves in GAUSS50 appear to be generated by diffractions of the P-diving wave, the direct wave root, and the S-diving wave. The amplitude characteristics, velocity, and particle motion of Stonely waves distinguish them from the other arrivals present. The amplitude of these interface waves dies off exponentially with distance away from both sides of the water-solid interface. Stoneley waves appear as small packets of energy propagating along the interface rather than as wave fronts travelling through the bodies of water or solid in the models (see figure 2.9). 
Figure 2.9. Numerical Schlieren diagrams of normalized compressional and shear energy for model GAUSS50. The velocity field has Gaussian autocorrelated random velocity variations with correlation length of 50 meters (see figure 2.6 for velocity field). The principle waves are very similar to those seen in the laterally homogeneous case of figure 2.7. Secondary effects include incoherent backscattering of $\mathrm{P}$ and $\mathrm{S}$ energy from the $\mathrm{P}$ and $\mathrm{S}$ diving waves and Stoneley wave generation along the interface from the transmitted waves and the direct wave root. Note the predominance of randomly scattered shear energy from both the S-diving wave and converted from the P-diving wave. Shear energy from the P-diving wave appears in front of the S-diving wave. 


\section{GAUSS50}
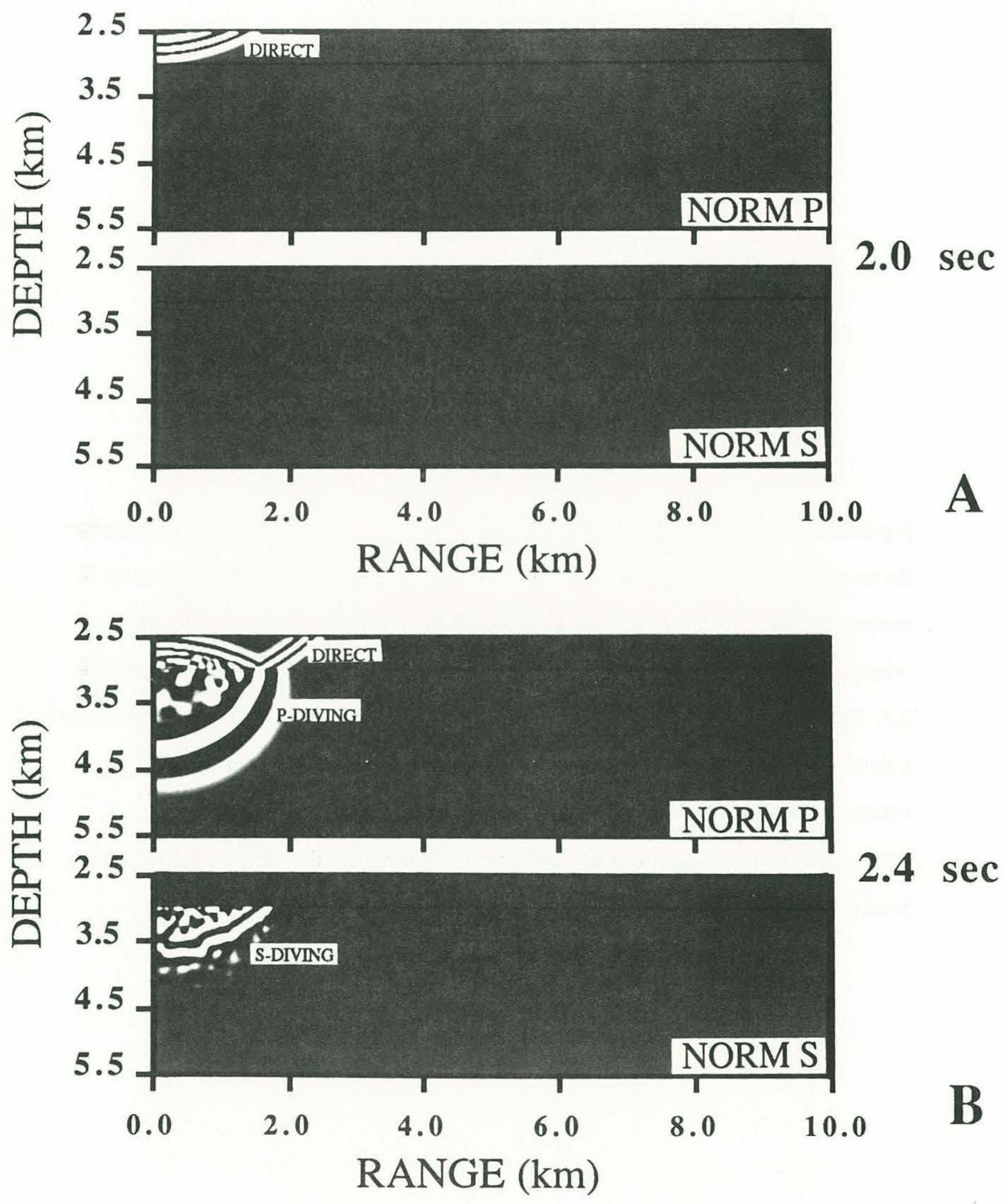
Figure 2.9. Numerical Schlieren diagrams of normalized compressional and shear energy for model GAUSS50. The velocity field has Gaussian autocorrelated random velocity variations with correlation length of 50 meters (see figure 2.6 for velocity field). The principle waves are very similar to those seen in the laterally homogeneous case of figure 2.7. Secondary effects include incoherent backscattering of $\mathrm{P}$ and $\mathrm{S}$ energy from the $\mathrm{P}$ and $\mathrm{S}$ diving waves and Stoneley wave generation along the interface from the transmitted waves and the direct wave root. Note the predominance of randomly scattered shear energy from both the S-diving wave and converted from the P-diving wave. Shear energy from the P-diving wave appears in front of the S-diving wave. 


\section{GAUSS50}
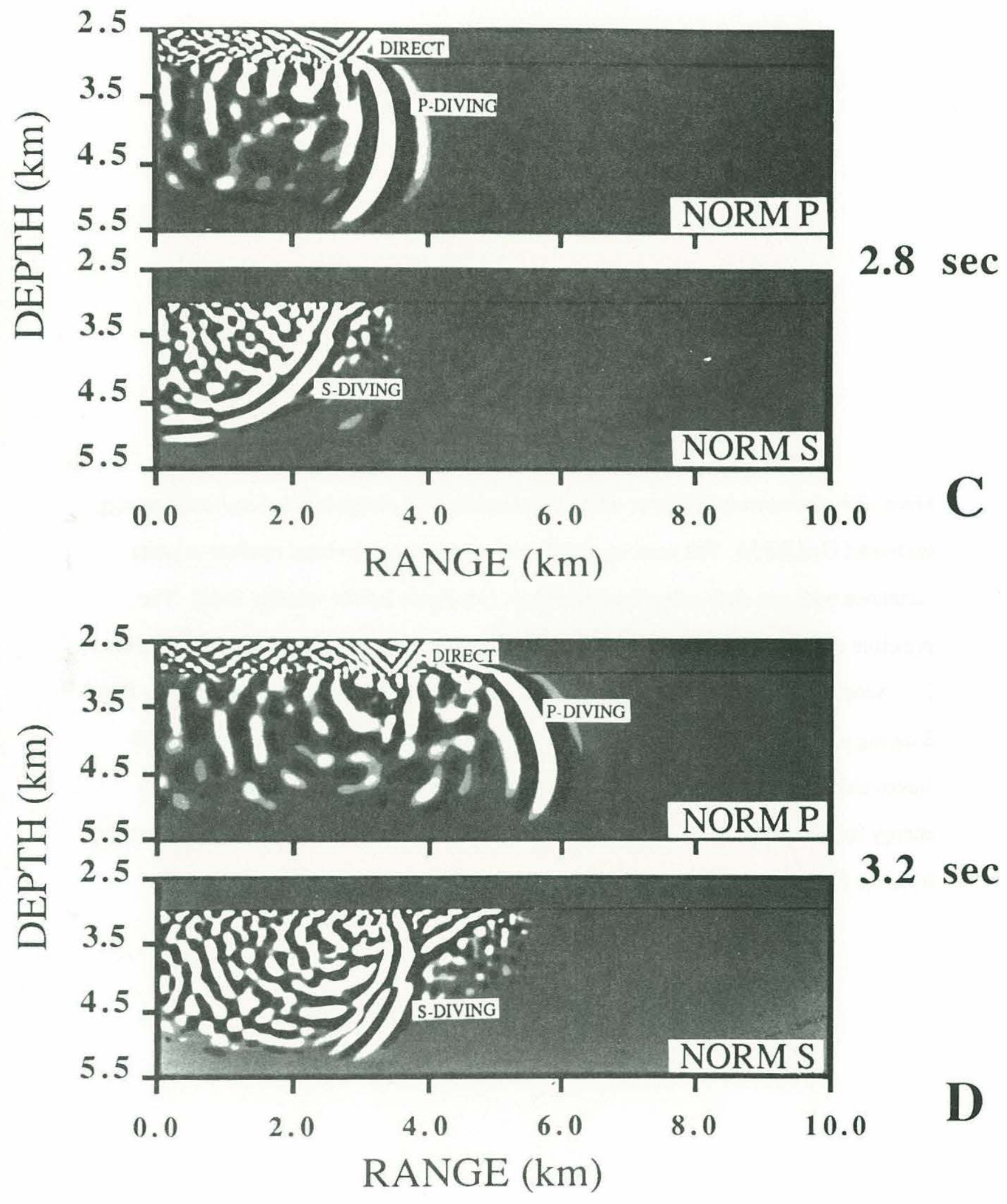
Figure 2.9. Numerical Schlieren diagrams of normalized compressional and shear energy for model GAUSS50. The velocity field has Gaussian autocorrelated random velocity variations with correlation length of 50 meters (see figure 2.6 for velocity field). The principle waves are very similar to those seen in the laterally homogeneous case of figure 2.7. Secondary effects include incoherent backscattering of $\mathrm{P}$ and $\mathrm{S}$ energy from the $\mathrm{P}$ and $S$ diving waves and Stoneley wave generation along the interface from the transmitted waves and the direct wave root. Note the predominance of randomly scattered shear energy from both the S-diving wave and converted from the P-diving wave. Shear energy from the P-diving wave appears in front of the S-diving wave. 


\section{GAUSS50}
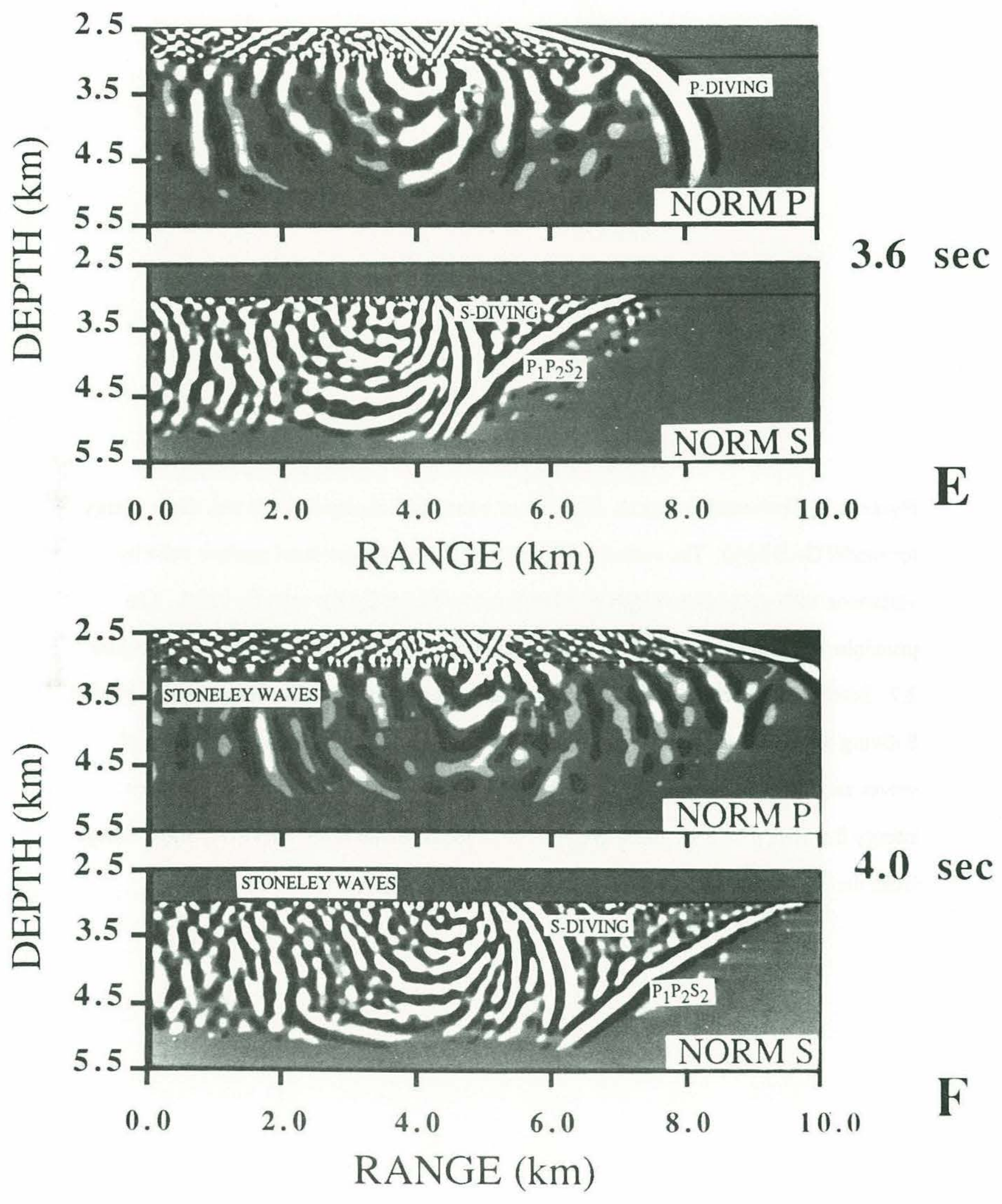
Figure 2.9. Numerical Schlieren diagrams of normalized compressional and shear energy for model GAUSS50. The velocity field has Gaussian autocorrelated random velocity variations with correlation length of 50 meters (see figure 2.6 for velocity field). The principle waves are very similar to those seen in the laterally homogeneous case of figure 2.7. Secondary effects include incoherent backscattering of $P$ and $S$ energy from the $P$ and $\mathrm{S}$ diving waves and Stoneley wave generation along the interface from the transmitted waves and the direct wave root. Note the predominance of randomly scattered shear energy from both the S-diving wave and converted from the P-diving wave. Shear energy from the P-diving wave appears in front of the S-diving wave. 


\section{GAUSS50}
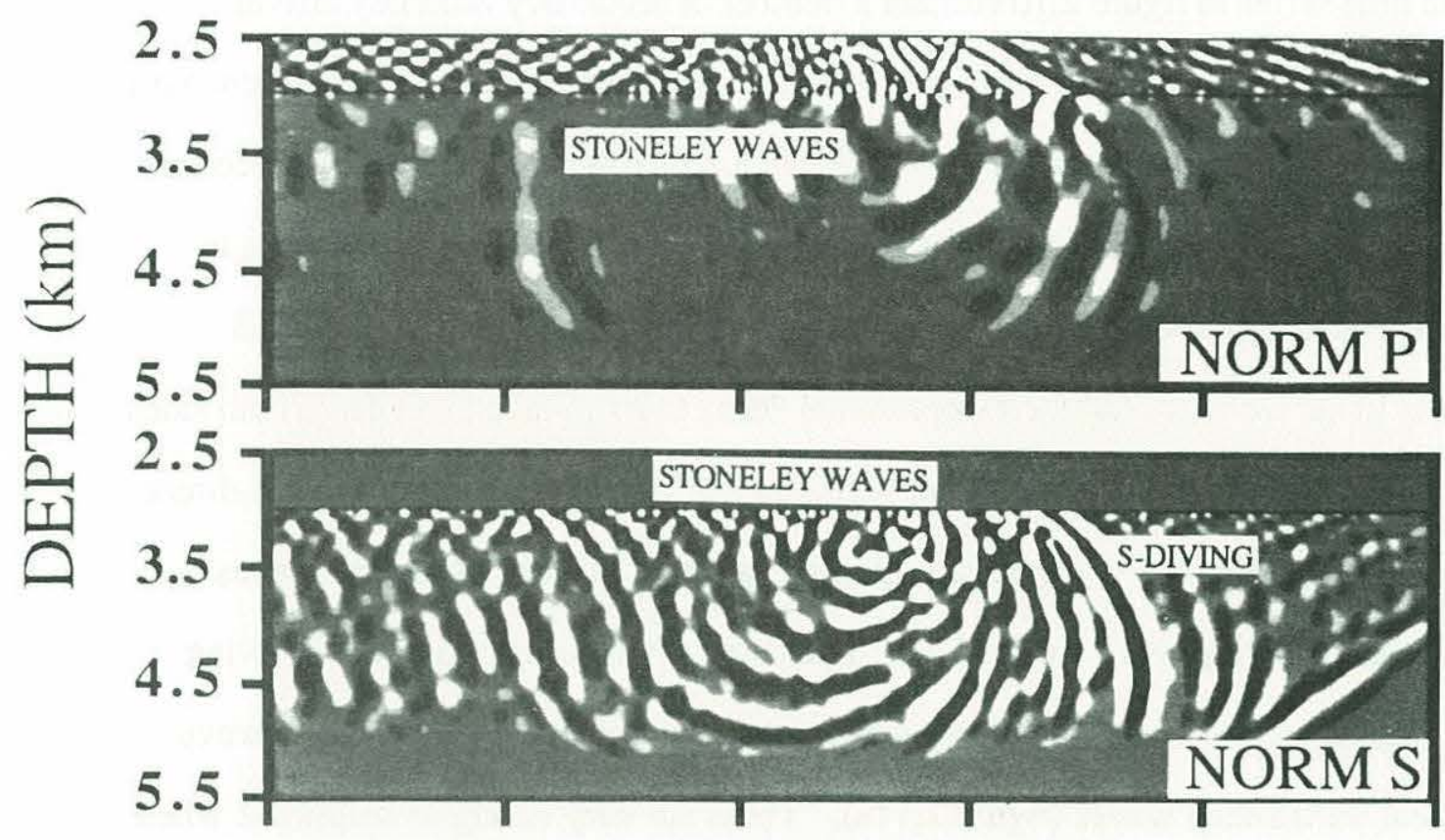

0.0

2.0

4.0

6.0

8.0

10.0

$4.4 \mathrm{sec}$

RANGE (km)
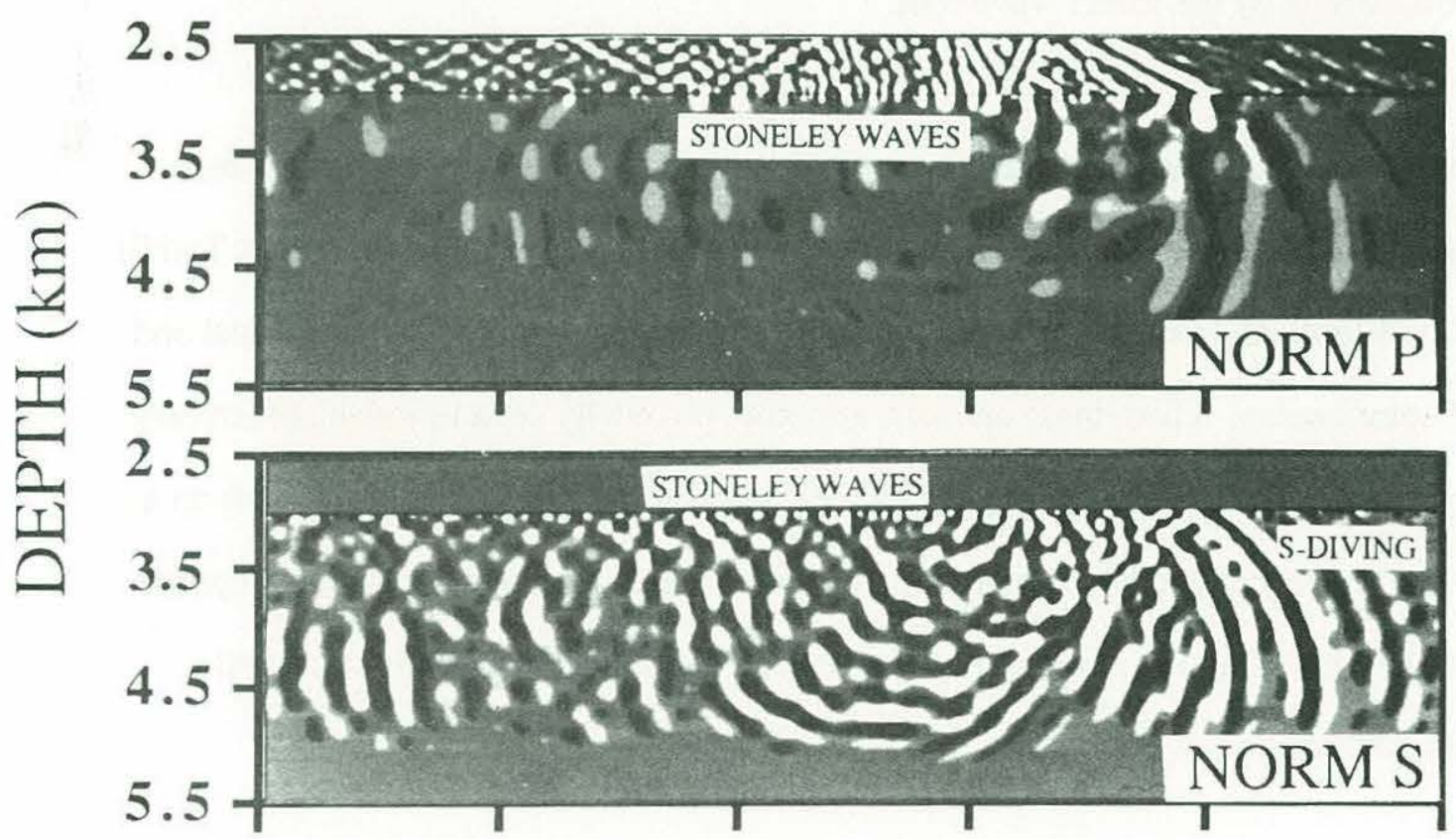
0.0
2.0
4.0
6.0
8.0
10.0
RANGE (km)

4.8 sec 
The time series in figure 2.10 contain a number of secondary Stoneley arrivals. Their low velocity and the number present (especially after the direct wave) make them hard to identify with the receiver spacing shown in figure 2.10. Stoneley wave arrivals become more apparent when signals from all of the receivers are plotted ( 40 meter spacing as in figure 2.11). The distinctive features of these arrivals are their low velocity (1.2-1.33 $\mathrm{km} / \mathrm{sec}$ ),their linear moveout and their exponential decay in amplitude away from both sides of the interface. Distinct Stoneley wave arrivals are seen following the P-diving and direct waves in 'blown-up' sections of the seismograms (figures 2.11a-b). Stoneley waves generated by the P-diving wave are weak but significant when compared to the P-diving amplitude (figure 2.11b). A number of Stoneley waves are generated by the direct wave root and shear transmitted waves (figure 2.11a). These are very strong in amplitude when compared with the P-diving wave and the Stoneley waves generated by it. Although most of the Stoneley waves are travelling to the right across the grid, there are a few which are scattered backwards by the direct wave root.

Secondary Stoneley waves have a characteristic elliptical, generally retrograde, particle motion at the water-solid interface (Bullen and Bolt, 1985, Schirmer, 1980, Tuthill et.al., 1981). Elliptical particle motion entails a 90 degree phase shift between vertical and horizontal components. Thus, these arrivals, although not easily seen as coherent arrivals when the receiver spacing is too great, can be identified by observing this phase shift on a superposition of vertical and horizontal particle motion traces. Also, when plotted against each other, the vertical and horizontal particle motions yield the charateristic retrograde elliptical particle motion (figure 2.12).

The 4.0 second snapshot for the other models with Gaussian velocity autocorrelation functions are shown in figure 2.13. The corresponding pressure time series 
are presented in figure 2.14. Again, as with GAUSS50, all of the expected, deterministic arrivals occur as well as a number of secondary scattered arrivals. In general, it appears that the amount of scattered energy decreases as $k a$ differs from one. Scattered arrivals become more coherent and deterministic as $k a$ increases ( $k a$ appr. equal to 4 for compressional waves in GAUSS200). Amplitude fluctuations and residual travel time of the initial P-diving wave also increase with increasing $k a$.

Model SELFSIM200, with a self-similar (fractal) velocity autocorrelation function is presented in figure 2.15. SELFSIM200, like GAUSS200 has a 200 meter correlation length and the large scale features of the snapshots and time series appear very similar between the two. However, the self-similar distribution also contains smaller scale fluctuations (see figure 2.6 for velocity fields) which scatter energy as in the Gaussian models with smaller correlation lengths. Although the principle effects of scattering seem to be characteristic of the large correlation length $(200 \mathrm{~m})$, some small correlation length effects are also present with the self-similar distribution. 
Figure 2.10. Synthetic seismograms for model GAUSS50. The major arrivals, as in the wavefront snapshots (fig. 2.9) are very similar to those of the laterally homogeneous case (fig. 2.8). a. Pressure signal from receivers 20 meters above the interface. b. Vertical displacement from receivers 30 meters below the interface. c. Horizontal displacement from receivers 30 meters below the interface. Only a few Stoneley waves are coherent with this receiver spacing (480 meters). 


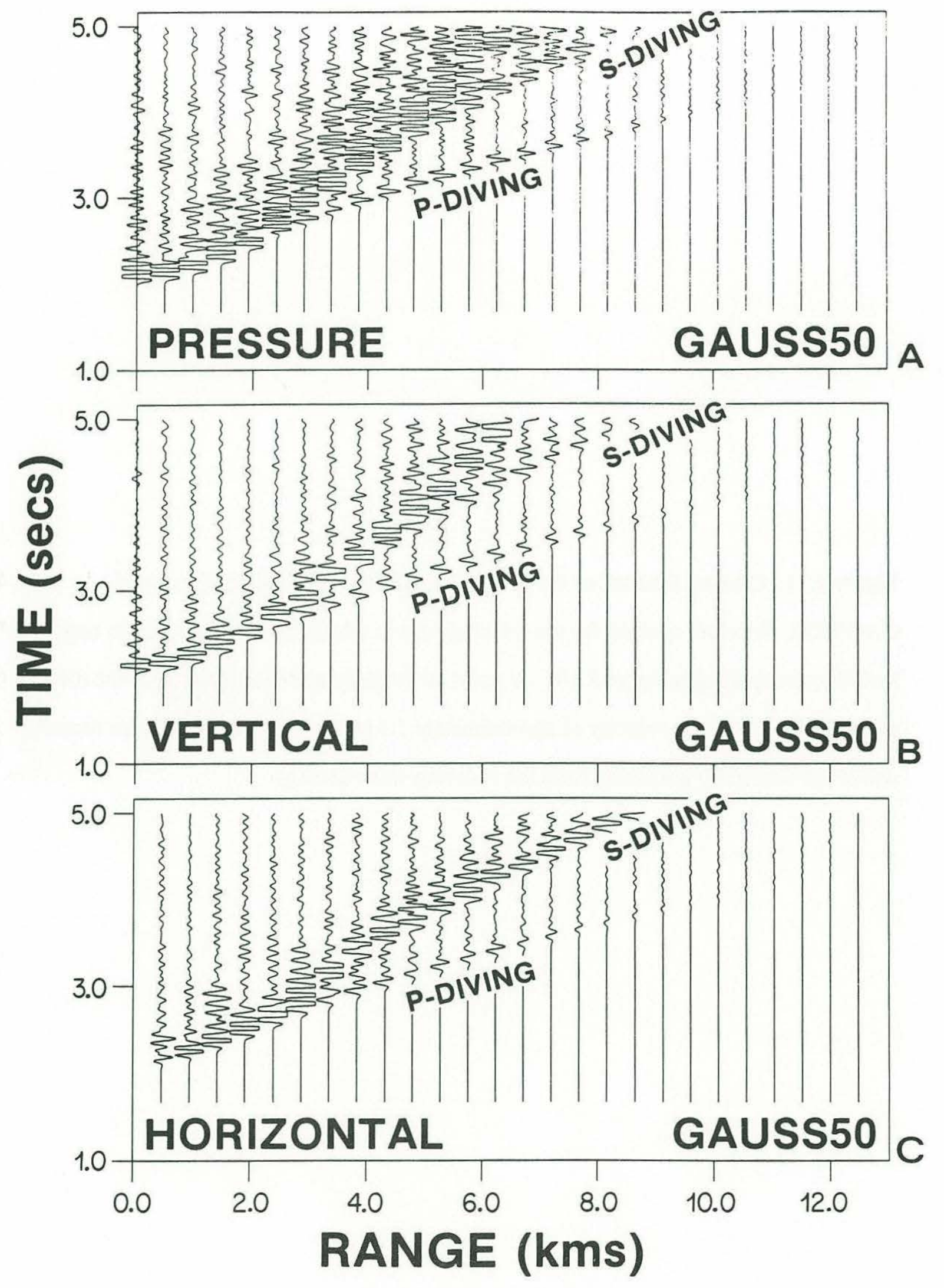


Figure 2.11. Closeup sections of the pressure synthetic seismograms for model GAUSS50. Receiver spacing for the seismograms in this figure is 40 meters (as opposed to 480 meter spacing in figure 2.10). A series of Stoneley arrivals appear after the direct wave and have a phase velocity of approximately $1.3 \mathrm{~km} / \mathrm{sec}$ (fig. $2.11 \mathrm{a}$ ). Other Stoneley waves are caused by scattering from the P-diving wave (2.11b). 


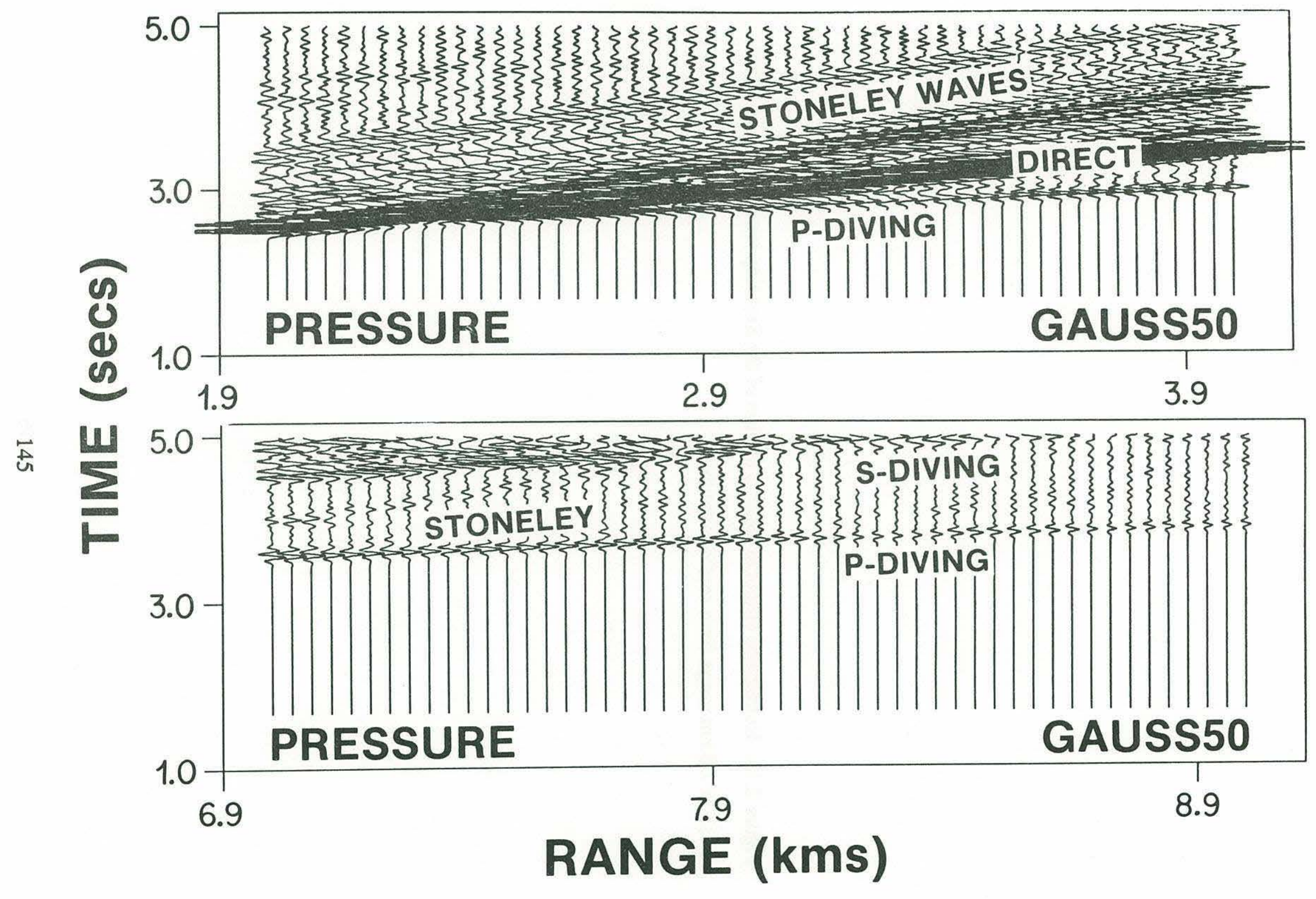


Figure 2.12. Retrograde elliptical particle motion of the Stoneley wave shown in figure $2.11 \mathrm{~b}$ at $7.5 \mathrm{~km}$ range for a seafloor receiver. 


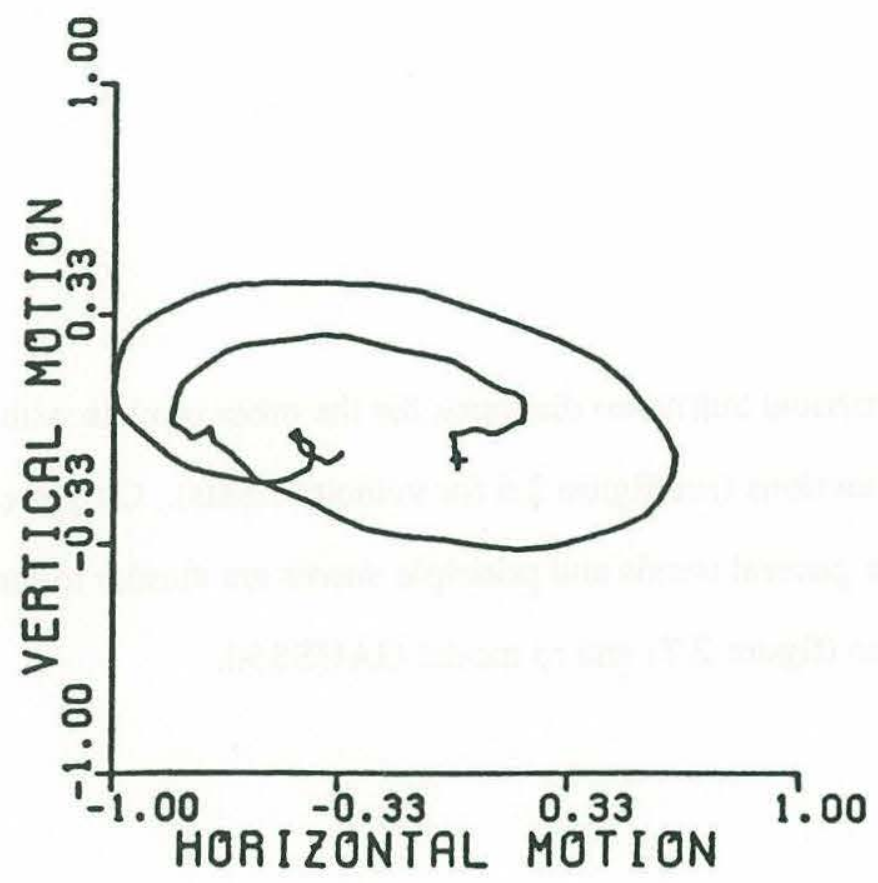


Figure 2.13. Numerical Schlieren diagrams for the other models with Gaussian autocorrelation functions (see figure 2.6 for velocity fields). Only the 4.0 second snapshot is shown since the general trends and principle waves are similar to those of the laterally homogeneous case (figure 2.7) and to model GAUSS50. 


\section{GAUSS10}
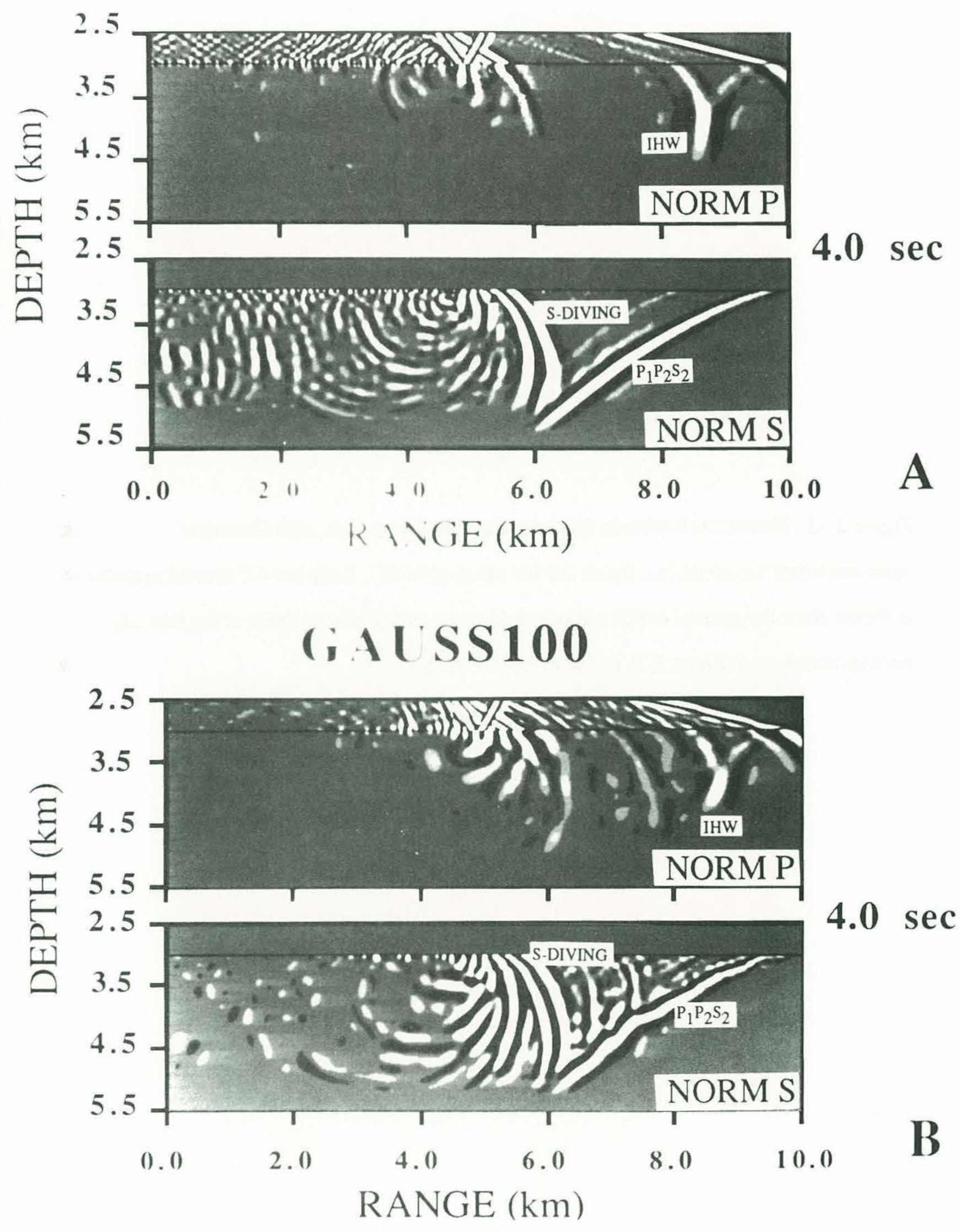
Figure 2.13. Numerical Schlieren diagrams for the other models with Gaussian autocorrelation functions (see figure 2.6 for velocity fields). Only the 4.0 second snapshot is shown since the general trends and principle waves are similar to those of the laterally homogeneous case (figure 2.7) and to model GAUSS50. 


\section{GAUSS200}

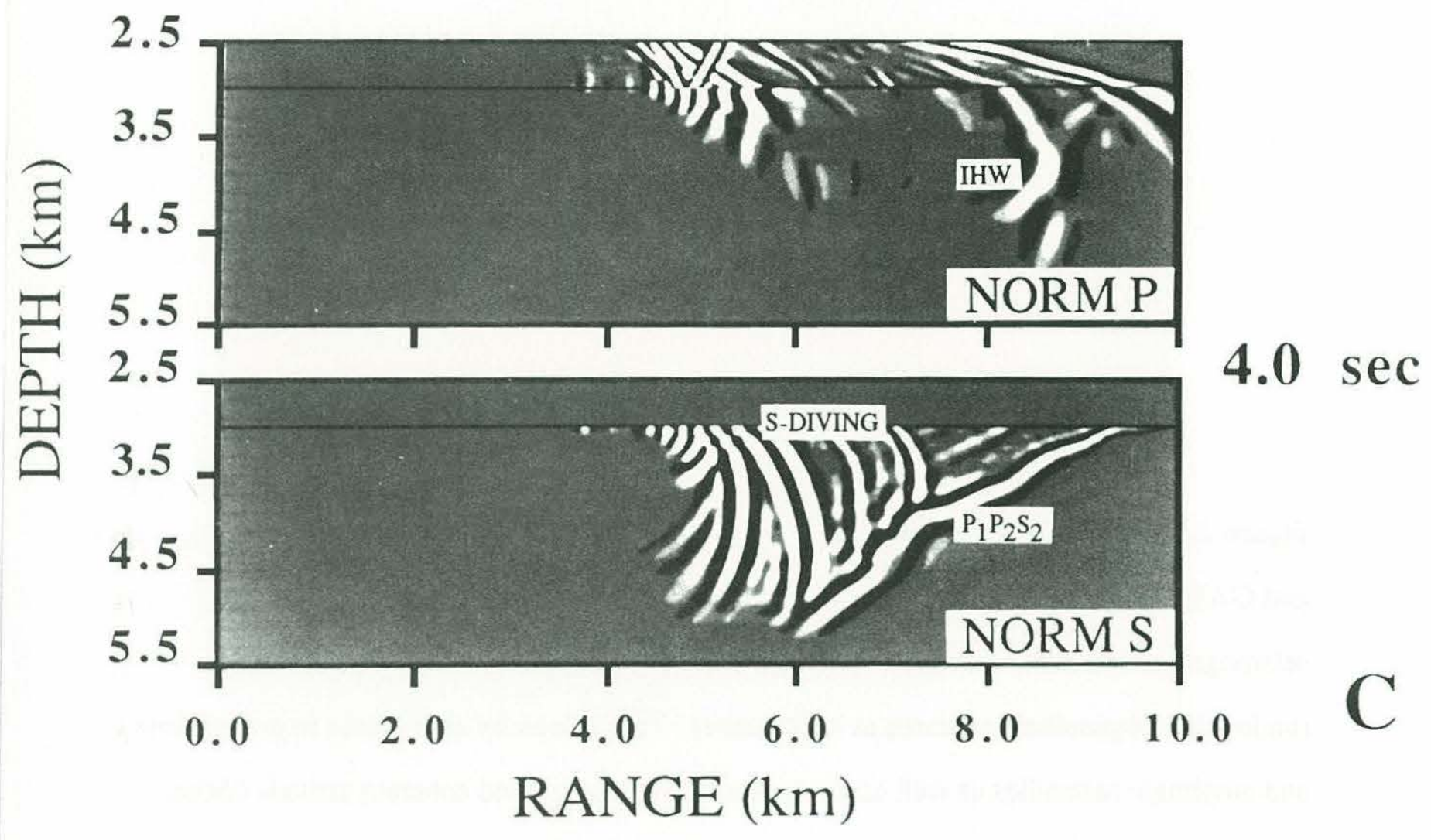


Figure 2.14. Pressure time series for models GAUSS10 (2.14a), GAUSS100 (2.14b), and GAUSS200 (2.14c). In general, the amount of random scatter, or 'noise', in the seismograms decreases as ka increases. Conversely, deterministic scattering from the random heterogeneities increases as ka increases. This is seen by an increase in arrival time and amplitude anomalies as well as the appearance of unexpected coherent arrivals (those not seen in the laterally homogeneous case) in the seismograms. 


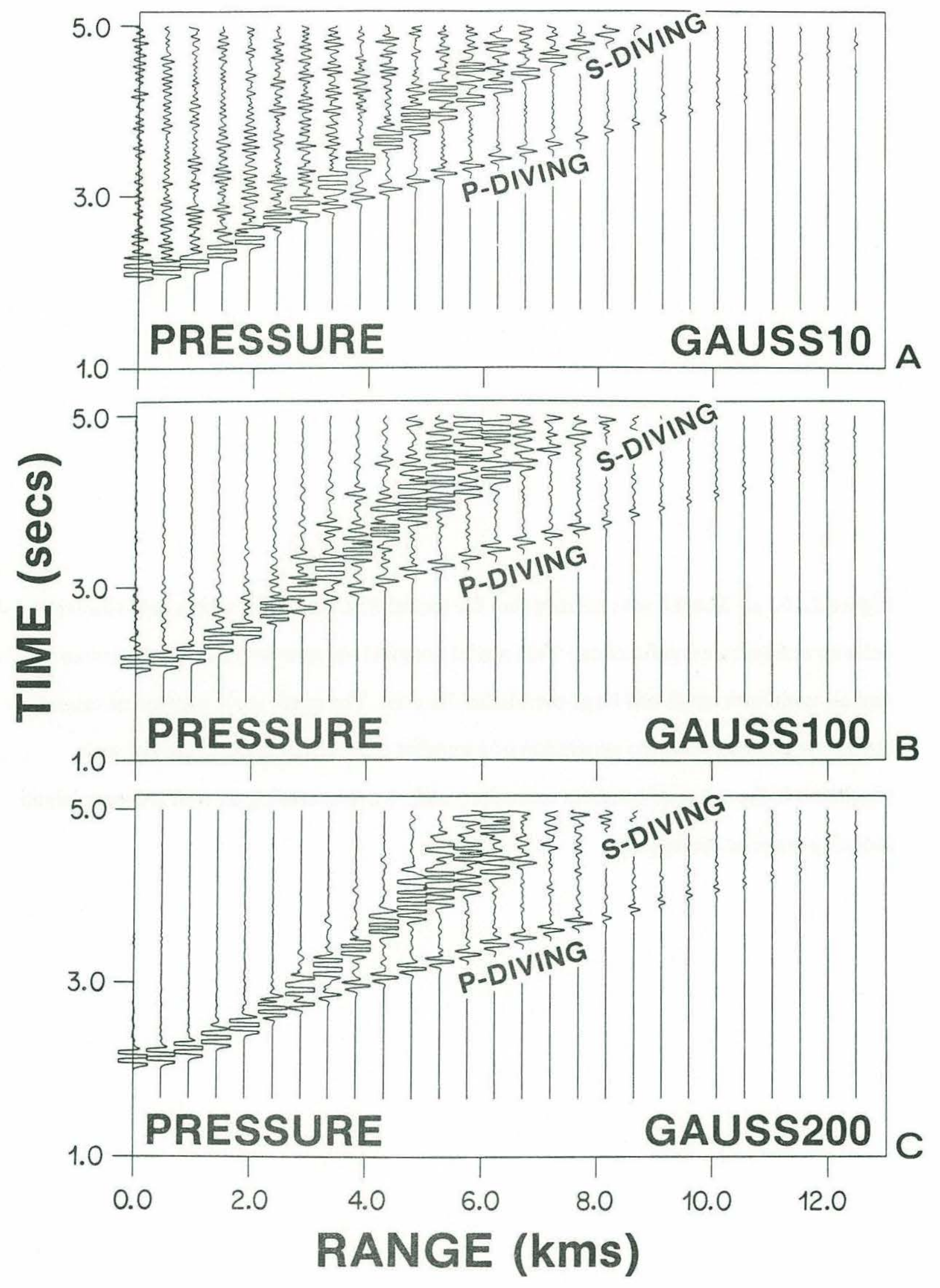


Figure 2.15. a. The 4.0 second snapshot for model SELFSIM200 with a self-similar velocity autocorrelation function. This model contains characteristics of the Gaussian models with both small and large correlation lengths. The small scale roughness causes random backscatter and the generation of a number of Stoneley waves. Larger scale roughness is the cause of coherent secondary body wave scattering as well a deterministic Stoneley wave scattering. 


\section{SELFSIM200}

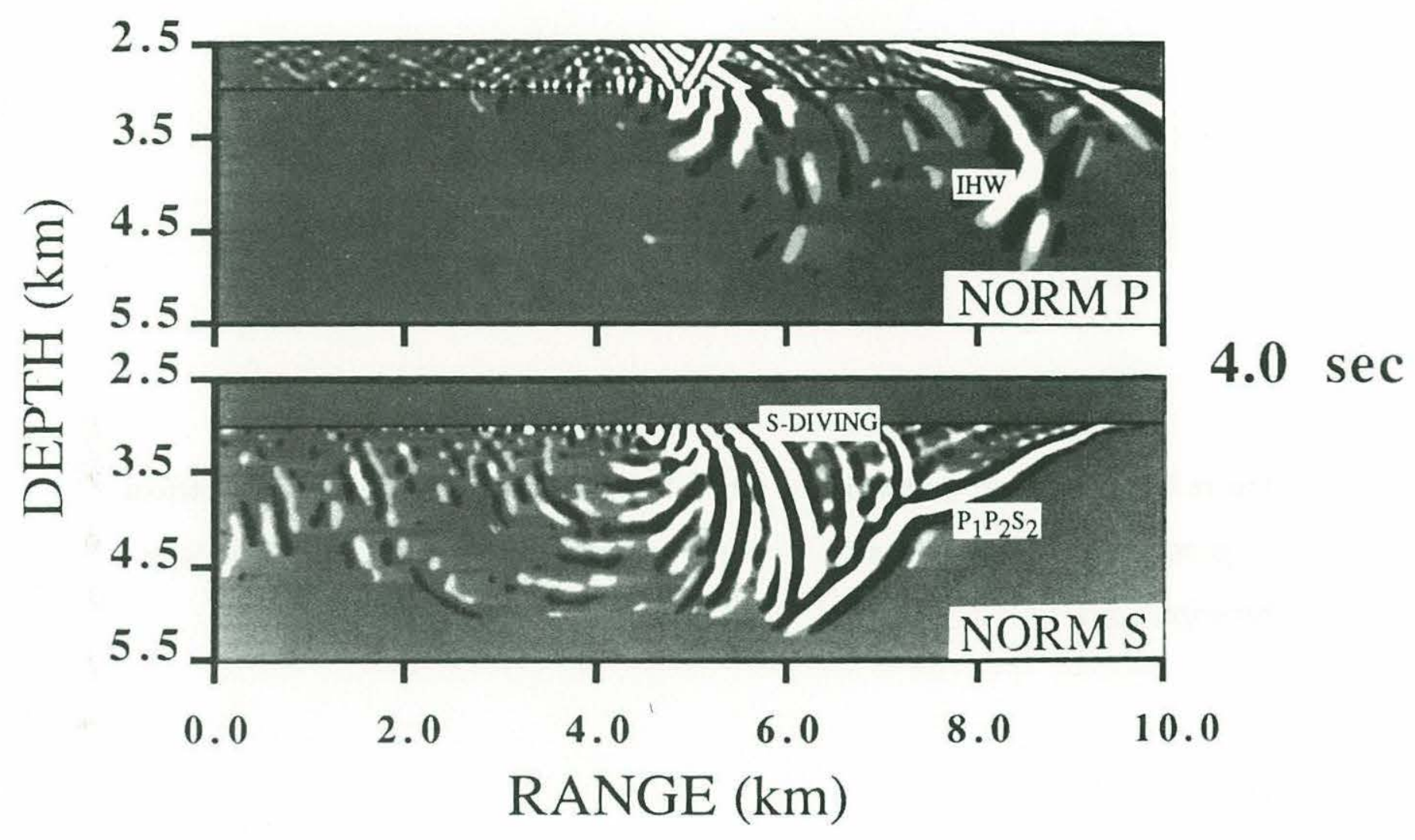


Figure 2.15 b. Pressure time series for model SELFSIM200. Again, as in the wavefront diagram, this model contains characteristics of scattering from both large and small scale heterogeneities. 


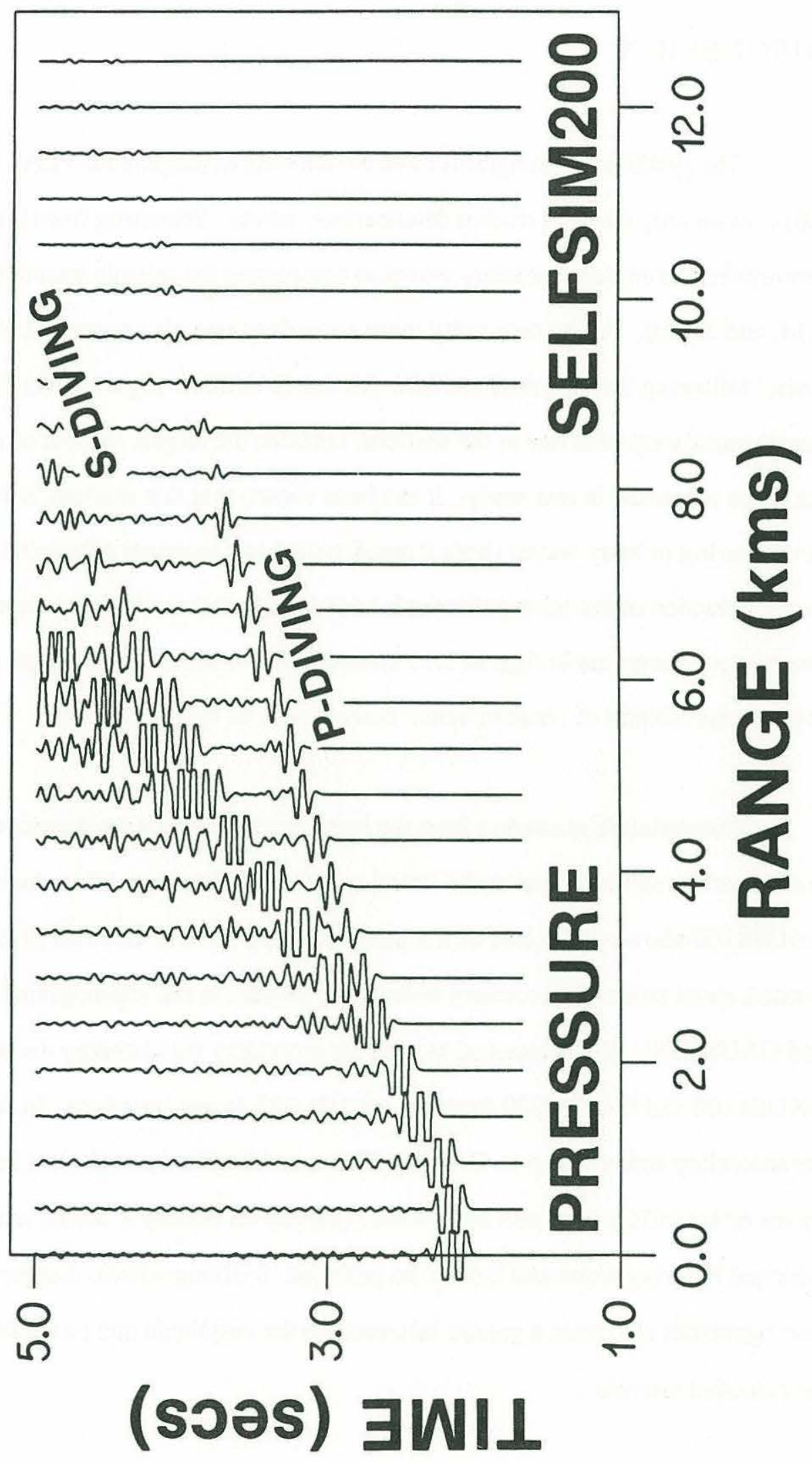




\section{DISCUSSION}

The synthetic seismograms from the laterally homogeneous FLAT model (figure 2.8) contain only the well studied deterministic waves. Scattering from lateral heterogeneities causes secondary waves to complicate the seismic traces (see figures 2.10, 2.14 , and $2.15 \mathrm{~b}$ ). For $k a$ near unity these secondary arrivals appear as random incoherent 'noise' following the principal arrivals. Model GAUSS50 (figure 2.10), with $k a$ approximately equal to one at the seafloor, contains the largest amount of random noise for the cases presented in this study. It has been shown that this random 'noise' is caused by the scattering of body waves (both $\mathrm{P}$ and $\mathrm{S}$ types) and secondary Stoneley wave generation from interaction of the principal arrivals with the heterogeneities. An inspection of the pressure seismograms in figures 2.10 and 2.14 shows that in general, for the Gaussian models, the amount of random 'noise' increases as $k a$ approaches one.

Deterministic scattering from the heterogeneities begins to dominate the secondary features of the seismograms as the lateral extent of the heterogeneities increases. Model GAUSS100 shows much less of the random 'noise' seen in GAUSS10 and GAUSS50. Instead, more coherent secondary arrivals are present in the seismograms of GAUSS100 and GAUSS200. The amount of incoherent secondary wave energy decreases in both GAUSS 100 and GAUSS200 from the models with lower $k a$ values. In fact, almost all of the secondary arrivals seen in GAUSS 200 can be identified as coherent secondary Stoneley waves or secondary scattered body waves (mainly secondary S waves arriving after the principal P-diving wave and before the principal S-diving wave). Larger scale heterogeneities also have a greater influence on the amplitude and travel time anomalies of the principal arrivals. 
One measure of the influence of lateral heterogeneities on the coherence of wave propagation is the cross correlation of seismogram arrivals at different ranges. Frankel and Clayton (1986) carried out this type of analysis on wave propagation through a halfspace with random heterogeneities. Our study is more complicated because of the presence of different phases arising from interaction of seismic energy with the liquid-solid interface. Therefore, before we can compare the cross-correlation patterns of different models with lateral heterogeneity, we must first understand the behavior of the cross-correlation analysis within the laterally homogeneous FLAT model at different ranges (and thus different wave relationships).

The normalized cross-correlation coefficient between a given trace and any other trace is equal to one for wave propagation through a homogeneous box. However, because of wave interaction with the liquid-solid interface, the synthetic seismograms in this study contain many different wave types on a given seismic trace. Because the different waves travel with different speeds and directions and the source is not equidistant from all of the receivers, a cross-correlation between any two entire traces is going to contain these deterministic effects. As the distance between traces increases, the crosscorrelation coefficient should roughly decrease because of unique characteristics of the different waves present. We have chosen to look at only the first 0.5 seconds of the initial P-wave arrival (the direct wave at small ranges, the P-diving wave at large ranges) for the cross-correlation analysis in order to minimize the artifacts of differences in the wave types.

Figure 2.16 is a plot of the cross-correlation coefficients between a number of traces $(0.5$ seconds after the P-diving wave first zero crossing) which are the seafloor pressure time series of model FLAT from 6.0 and $9.0 \mathrm{~km}$. (see labels on curves in figure 2.16) and all of the other traces within this synthetic seismogram between 6.0 and $9.0 \mathrm{~km}$. This figure demonstrates that the correlation between P-diving waveforms is range 
Figure 2.16. Cross correlation coeficients for the synthetic seismogram traces between 6.0 and $9.0 \mathrm{~km}$. of model FLAT. The curves shown are for a number of reference traces (labelled) correlated with all other traces between 6.0 and $9.0 \mathrm{~km}$. Spatial lag is the difference between the range of the reference trace and that of the cross-correlation trace. Correlation analysis was done for the first 0.5 seconds after the P-diving wave first zero crossing. Note that the correlation coefficient falls off more rapidly for reference traces at relatively greater ranges. 


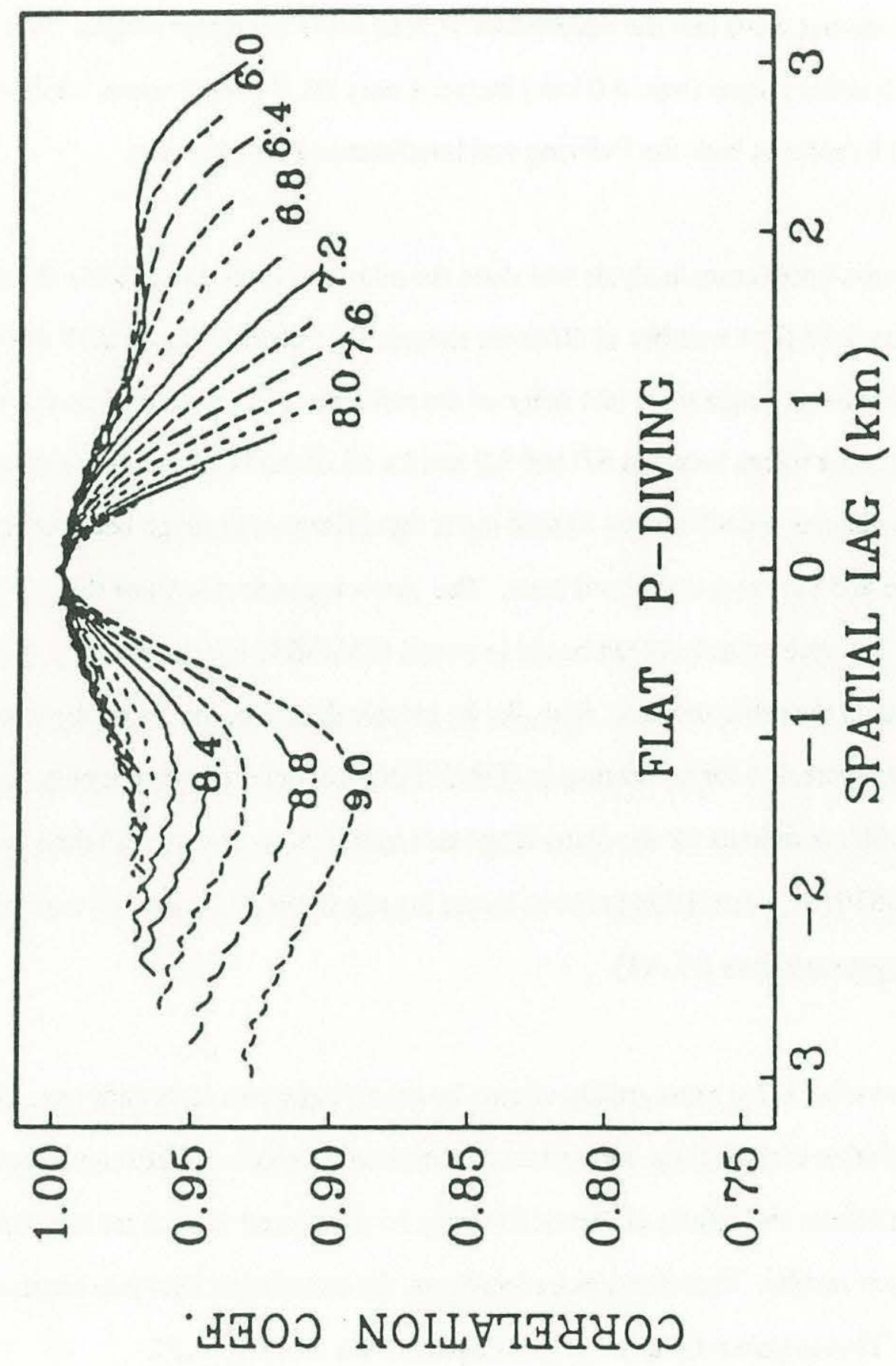


dependent even for this laterally homogeneous case. For example, the correlation between the $9.0 \mathrm{~km}$. trace and its neighbors decreases with range faster than the correlation between the $6.0 \mathrm{~km}$. trace and its immediate neighbors. This is due to the increase of interaction between the $\mathrm{P}$-diving wave and the interference P-head wave at greater ranges. The crosscorrelation at smaller ranges (near $6.0 \mathrm{~km}$.) includes only the P-diving wave, while at greater ranges it includes both the P-diving and interference P-head waves.

This cross-correlation analysis was done for all of the Gaussian models and is shown in figure 2.17 for a number of different ranges. The plots in figure 2.17 are crosscorrelations between a single trace (the range of the reference trace is labeled on top of each frame) and all other traces between 6.0 and $9.0 \mathrm{~km}$ for all of the Gaussian models and the laterally homogeneous FLAT model. Spatial lag is the difference in range between the reference trace and the cross-correlated trace. The general pattern for all of the ranges shown is that the correlation between traces in model GAUSS50 ( $k a$ approximately equal to one) is lower than the other models. Also, for $k a$ greater than one, the correlation between traces generally increases for increasing $k a$ (GAUSS200 coeficients are generally higher than GAUSS100 coeficients for the same range and spatial lag). For $k a$ less than one (model GAUSS10) the correlation between traces is only slightly less than that of the laterally homogeneous case (FLAT).

The waveforms are most greatly altered by scattering when $k a$ is near one. Since the cross-correlation computation normalizes for amplitude variations between traces and travel time variations, the effects observed for large $k a$ (discussed above) are not included in the correlation results. Therefore, as $k a$ increases, the correlation between traces should also increase. This is generally true for the ranges shown in figure 2.17. 
Although significant 'coda' exists for model GAUSS10, suggesting that random scatter is important, there is apparently little change in the waveform caused by the small scatterers. Since $k a$ is small for GAUSS10, the wave field is starting to see the velocity perturbations as an equivalent body with an average velocity field approaching that of the FLAT model. The random noise is slightly higher in frequencey and arises from scattering of the higher frequency components of the source. The predominantly $10 \mathrm{~Hz}$ P-diving wave is not greatly affected.

The distinction between the cross correlation characteristics of the different models decreases as the range of the reference trace increases. A clear separation exists between the cross-correlation patterns of the different models if the reference trace is at a relatively small range (such as $6.0 \mathrm{~km}$. in figure 2.17a.). As the range of the reference trace increases there is an overall decrease in most of the absolute values of the correlation coeficients as well as a decrease in the distinction of the patterns between models. This is probably due to the influence of the interference head wave at greater ranges. That is, low correlation between traces is influenced more by the interference head wave than by the heterogeneity at large ranges. Model GAUSS50 which has a lower correlation curve than the others has about the same correlation values with different reference ranges because the large amount of scattering causes the interference head wave to be at a low amplitude already (see figures 2.10 and 2.14 for a comparison of interference head wave amplitudes). Models with less random scatter have stronger interference head waves at large ranges so that the decrease in correlation coefficients with range is larger for these models. 
Figure 2.17. Comparison of the cross-correlation curves for all Gaussian models and the laterally homogeneous model at 4 different reference trace ranges. a. Reference trace at $6.0 \mathrm{~km}$. There is a clear distinction between the curves for the different models at this range. b. $7.0 \mathrm{~km}$. reference trace. c. $8.0 \mathrm{~km}$. reference trace. d. $9.0 \mathrm{~km}$. reference treace. The absolute values for the GAUSS50 curve have remained about the same for the 4 different reference ranges presented. However, the overall values for the other curves have decreased with increasing range so that by $9.0 \mathrm{~km}$. it is difficult to notice any major distinctions between the 5 curves. 
A

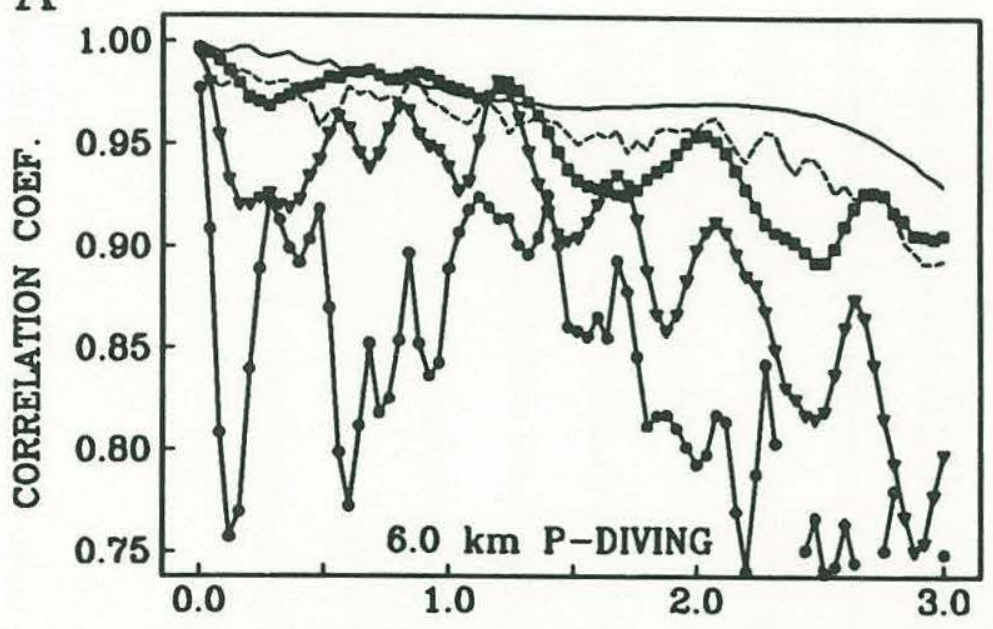

a

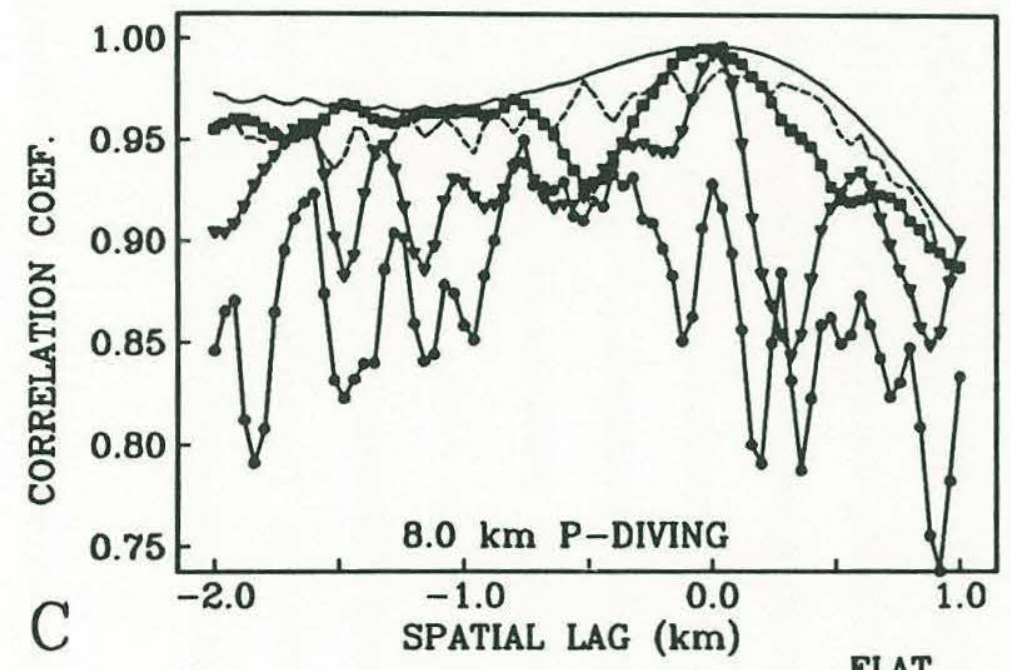

FLAT

GAUSS 10

GAUSS50

GAUSS $100^{\circ}$

GAUSS200"
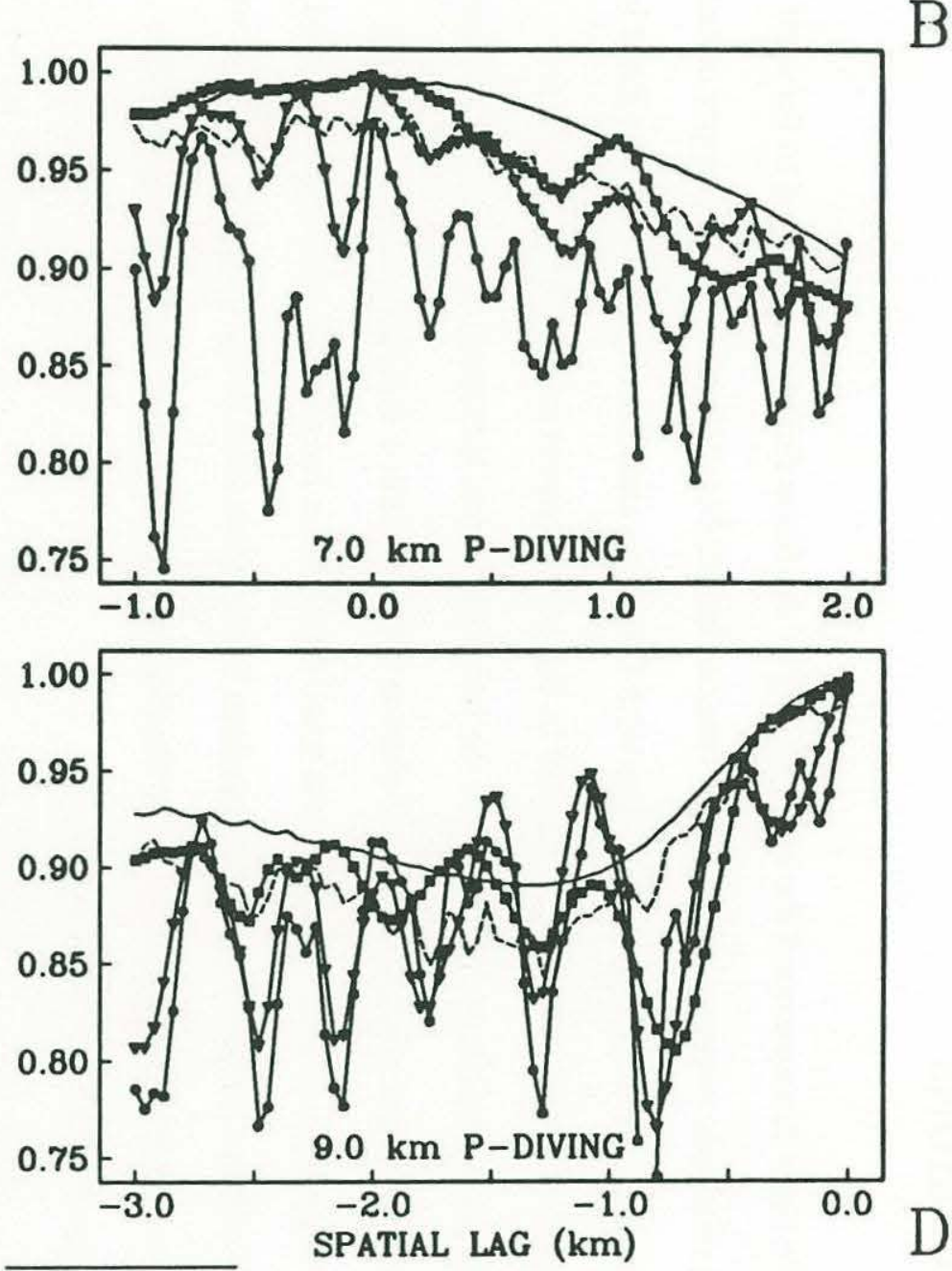


\section{CONCLUSIONS}

We have demonstrated the applicability of the finite difference method to forward modeling of elastic wave scattering from random lateral heterogeneities in the upper oceanic crust. For random velocity perturbations with Gaussian autocorrelation functions there is a general trend of increasing random scatter in the seismograms as $k a$ approaches one.

Secondary deterministic scattering from the heterogeneities increases as $k a$ increases from one. Velocity perturbations with a self-similar autocorrelation function cause scattering with characteristics of both the large and small correlation length Gaussian models. That is, the self-similar model contains the random backscatter seen in the Gaussian models with $k a$ less than one as well as the effects of travel time and amplitude anomalies seen in the Gaussian models with $k a$ larger than one.

Heterogeneities in the upper crust near the water-solid interface act as sources for secondary Stoneley waves when illuminated by primary seismic energy. The presence of the water-solid interface in the models is important because the majority of the seafloor 'noise' seen in the synthetic seismograms seems to travel as secondary Stoneley waves. Simple models of propagation through a half-space (e.g. Frankel and Clayton, 1986, McLaughlin et.al., 1985) do not include interface phenomena which are an integral part of the marine problem. Also, inclusion of the water-solid interface allows the examination of the influence of scattering on the many different wave types which arise from interaction with the interface. The interference P-head wave has a significant influence on the crosscorrelation between traces in the scattering models.

Cross-correlation analysis of the P-diving wave in the scattering models reveals two important characteristics. First, as $k a$ approaches one from below, the correlation between 
seismic traces decreases. Deterministic scattering from spatially larger heterogeneities $(k a>1)$ causes much less decorrelation of the traces than does 'random' scattering from heterogeneities with $k a$ near one. Second, the cross-correlation values are dependent upon the range of the reference trace of the analysis. This is due to the influence of the interference head wave at large ranges. 


\section{REFERENCES}

Aki, K., 1973, Scattering of P-waves under the Montana Lasa, J. Geophys. Res., 1346.

Aki, K., 1982, Scattering and attenuation, Bull. Seism. Soc. Am., v.72, p.319--330.

Alford, R.M., Kelly, K.R., and Boore, D.M., 1974, Accuracy of finite difference modeling of the acoustic wave equation, Geophysics, v.39., p.834-842.

Alterman, Z., and Loewenthal, D., 1972, Computer generated seismograms, in Methods in Computational Physics (Bolt, B.A., ed.), v.12, Academic Press, New York.

Bhasavanija, K., 1983, A finite difference model of an acoustic logging tool: The borehole in a horizontally layered geologic medium, Ph.D. thesis, Colorado School of Mines, Golden, Colorado.

Brekhovskikh, L.M., 1960, Waves in Layered Media, Academic Press, New York, 503p.

Bullen, K.E., and Bolt, B.A., 1985, An Introduction to the theory of Seismology, Cambridge University Press, New York, 498 p.

Cerjan, Charles, Kosloff, Dan, Kosloff, Ronnie and Reshef, Moshe, 1985, A nonreflecting boundary condition for discrete acoustic and elastic wave equations, Geophysics, v.50, p.705-708.

Cerveny, V., and Ravindra, R., 1971, Theory of Seismic Head Waves, University of Toronto Press, Toronto, pp.235-250.

Chernov, L.A., 1960, Wave Propagation in a Random Medium, McGraw-Hill Book Co., New York, 168p.

Clayton, R., and Engquist, B., 1977, Absorbing boundary conditions for acoustic and elastic wave equations, Bull. Seis. Soc. Am., v.67, p.1529-1540.

Dougherty, M.E., and Stephen, R.A., 1987, Geoacoustic scattering from seafloor features in the ROSE area, J. Acoust. Soc. Am., v.82, p.238-256.

Ewing, J.I., and Purdy, G.M., 1982, Upper crustal velocity structure in the ROSE area of the East Pacific Rise, J. Geophys. Res., v.87, p.8397-8402.

Fornberg, B., 1987, The pseudospectral method: Comparisons with finite differences for $\mathrm{t}$ he elastic wave equation, Geophysics, v.52, p.483-501.

Frankel, Arthur, and Clayton, Robert W., 1986, Finite difference simulations of seismic scattering: Implications for the propagation of short-period seismic waves in the crust and models of crustal heterogeneity, J. Geophys. Res., v.91, p.6465-6489.

Hudson, J.A., and Heritage, J.R., 1981, The use of the Born approximation in seismic scattering problems, Geophys. J. R. astr. Soc., v.69, p.649-657. 
Kelly, K.R., Ward, R.W., Treitel, S., and Alford, R.M., 1976, Synthetic seismograms: A finite difference approach, Geophysics, v.41, p.2-27.

Levander, A.R., 1985, Use of the telegraphy equation to improve absorbing boundary efficiency for fourth-order acoustic wave finite difference schemes, Bull. Seism. Soc. Am., v.75, p.1847-1852.

Mandelbrot, B.B., 1977, Fractals, form, chance, and dimensions, W.H. Freeman and Company.

McLaughlin, K.L., 1983, Spatial coherency of seismic waveforms, Ph. D. thesis, Univ. of California-Berkeley, 275p.

McLaughlin, K.L., Anderson, L.M., and Der, Z.A., 1985, Investigation of scattering and attenuation of seismic waves using 2-dimensional finite difference calculations, presented at Symposium on scattering of waves in random media and random rough surfaces, Pennsylvania State University.

Morse, P.M., and Feshbach, H., 1953, Methods of Theoretical Physics, McGraw-Hill Book Company, New York.

Nafe, J.E., and Drake, C.L., 1957, Variations with depth in shallow and deep water marine sediments of porosity, density, and the velocities of compressional and shear waves, Geophysics, v.22, p.523-552.

Nicoletis, L., 1981, Simulation numerique de la propagation d'ondes sismiques dans les milieux stratifies a deux et trois dimensions: contributions a la construction et a l'interpretation des sismogrammes synthetiques, Ph. D. thesis, Universite Pierre et Marie Curie, Paris, France.

Purdy, G.M., 1982, The variability in seismic structure of layer 2 near the East Pacific Rise at 12 N, J. Geophys. Res., v.87, p.8403-8416.

Sato, H., 1982, Attenuation of S-waves in the lithosphere due to scattering by its random velocity structure, J. Geophys. Res., v.87, p.7779-7786.

Schirmer, Florian, 1980, Experimental determination of properties of the Scholte wave in the bottom of the North Sea, in Bottom-interacting Ocean Acoustics, ed.

Kuperman, W.A., and Jensen, F.B., Plenum Press, New York, p.285-298.

Stephen, R.A., 1988, A review of finite difference methods for seismo-acoustic problems at the sea floor, Reviews of Geophysics, v.26, p.445-458.

Stephen, R.A., 1988, Lateral heterogeneity in the upper oceanic crust at DSDP Site 504, J. Geophys. Res., v. 93, p.6571-6584.

Stephen, R.A., Pardo-Casas, F., and Cheng, C.H., 1985, Finite difference synthetic acoustic logs, Geophysics, v.50, p.1588-1609.

Stephen, R.A., and Bolmer, S.T., 1985, The direct wave root in marine seismology, Bull. Seis. Soc. Am., v.75, p.57-67.

Tuthill, J.D., Lewis, B.R., and Garmany, J.D., 1981, Stonely waves, Lopez Island noise, and deep sea noise from 1 to $5 \mathrm{hz}$, Marine Geophysical Researches, v.5, p.95-108. 
Virieux, J., 1986, P-SV wave propagation in heterogeneous media: Velocity-stress finite difference method, Geophysics, v.51, p.889-901. 
CHAPTER 3

Seismo/acoustic propagation through rough seafloors 


\section{INTRODUCTION}

The problem of seismic energy scattering from heterogeneities within the crust is complicated by the presence of topography on the seafloor or sediment/basement interface. While heterogeneities within the crust can contain velocity contrasts on the order of 1-20 per cent, the water-solid interface can represent a velocity contrast of over 100 per cent. Because of this sharp impedance contrast, non-planar seafloors can cause large scattering effects when energy propagates into the oceanic crust. With increased interest in the seismic study of mid-ocean ridges where the impedance contrast is particularly high, it is important to understand the relationship between topography and seismo/acoustic propagation through the crust.

Topography of the seafloor covers a wide spectrum of wavenumbers. While the general impression of the seafloor is one of a deep, flat, sediment covered abyssal plane, Menard (1964) points out that most of the world ocean floor is indeed covered by rough, hilly terrain (as much as $85 \%$ of the Pacific ocean floor may have this type of terrain). Early surveys of compilations of the statistics of ocean floor topography showed a very red spectrum (Bell, 1975; Bell, 1979), that is, one which is weighted heavily in the longer wavelengths. However, inclusion of more recent deep-tow and mid-ocean ridge data will add at least some power to the spectrum at higher wavenumbers. Regardless, there is enough topography with wavenumbers similar to those of seismic energy to merit study of the propagation effects of topography. No section of the ocean floor is perfectly smooth, but rather, each contains a range of topographic length scales. The section of the range of length scales seen will depend on the resolution of the detection method used (Akal, 1978; Ogilvy, 1987). Seismic energy will be most effective in detecting topography which has length scales similar to and larger than that of the seismic wavelength. 
Acoustic scattering from rough surfaces is, in general, a very well studied problem. Many approximations exist to calculate the field scattered from a rough bottom (Ogilvy, 1987). Well known solutions which contain restrictive approximations include Rayleigh's method of plane wave summation (Rayleigh, 1878), the method of small perturbations (very small topography with respect to acoustic wavelength), the Kirchhoff method (assumes shallow slopes), and methods which sum contributions from distributions of points sources, facets, or protuberances along the bottom (see Ogilvy, 1987).

Recently, methods have been developed which are more flexible and require fewer limiting assumptions and give more realistic results. Bouchon (1985) used a boundary integral formulation coupled with the discrete wavenumber method to calculate the scattered field emanating from a rough surface of arbitrary wavenumber. This method has been successfully used to model diffraction of energy from a corrugated boundary (Campillo and Bouchon, 1985; Paul and Campillo, 1988). The emergence of more powerful computers has made the use of numerical methods such as the finite difference and finite element methods much more attractive because of the complete solution to the scattering problem with very few limiting assumptions. The major limitation of these methods is that they are computationally intensive and unpractical for very long range models. However, these methods are well suited for investigating the local effects of scattering at the sea floor. Stephen (1984) used the finite difference method to explain double head waves caused by seafloor topography and Dougherty and Stephen (1987, chapter 1) used this method to study 'refraction branch diffractions' from seafloor features beyond the critical range. (Hill and Levander, 1984; Levander and Hill, 1985) also used the finite difference method and investigated scattering from rough low velocity layers within the crust and rough elastic surface layers. 
The work for this chapter involves the use of a velocity-stress formulation of the finite difference method for elastic wave propagation (Dougherty and Stephen, 1988 (chapter 2); Stephen, 1988; Virieux, 1986) to investigate scattering from rough seafloor topography. While the long term goal of this work is to be able to distinguish between scattering from volume and surface heterogeneities, this chapter deals mainly with procedural problems with the method. Briefly, most of the difficulty in using this method for modelling smoothly varying topography lies in the definition of a non-planar, curvilinear seafloor on a rectangular grid. For rectangular grids which are numerically stable, the discretization of the seafloor profile imposes a stair-stepping microroughness onto the larger scale sinusoidal topography. Scattering phenomena due to this problem will be discussed as well as 'real' effects which can be expected from sinusoidal topography. 


\section{INITIAL MODELS}

The goal of this and future work on scattering from rough surfaces is to provide insight into the distinction between topographic and volumetric scattering mechanisms and effects. There are obviously an infinite number of topographic profiles present in the oceans so we have no hope of modelling every specific case. The best that can be done is

to quantify characteristics of scattering from generalized seafloor structures. In an effort to start this process, this chapter deals with a number of rough seafloor models which contain sinusoidal topography with height on the order of the seismic wavelength. While the existence of exactly this type of topography is highly unlikely, these models do provide a good starting point for the problem and are useful in that they point out both some important scattering mechanisms as well as operational constraints on the method used.

Sinusoidal seafloor profiles were chosen for this first set of models for a number of reasons. The regular nature of the sine wave makes the topography and underlying velocity structure easy to define on the finite difference grid. Also, the regularity of the sine functions allows for easy modification of height and wavelength parameters. The disadvantage of using a regular structure is that Bragg interference effects may become an issue. Another reason why the sine wave was chosen was because it provides for an easily defined, smoothly varying bottom. The effects of sharp breaks (such as the quarter space problem) have been investigated by a number of different authors (Achenbach et al., 1982; Kelly et al., 1976; Virieux, 1984; Virieux, 1986). Finally, in a very rough sense, sinusoidal topography does approximate linear, ridge-parallel, seafloor structures near midocean ridges. 
Elastic wave propagation through a number of rough seafloor models was calculated by using a 2-D elastic finite difference formulation of the elastodynamic wave equations for heterogeneous media. This formulation is well suited to handle laterally heterogeneous media and the interface between a liquid and solid present in marine models (Dougherty and Stephen, 1988 (chapter 2); Stephen, 1988b; Virieux, 1986). More detail on the specific formulation used can be found in Dougherty and Stephen (1988, chapter 2) and Stephen (1988a). A diagram of the grid layout is presented in figure 3.1. A rectangular grid with vertical and horizontal spacing of 10 meters was used for most of the models presented in this chapter. Outgoing energy is absorbed on the top, bottom and right hand sides of the grid (Dougherty and Stephen, 1988 (chapter 2); Stephen, 1988c, Clayton and Engquist, 1977), while the left hand side acts as an axis of symmetry. The sinusoidal profiles have a phase shift of $-\pi / 2$ to avoid symmetry problems at zero range. Two rows of receivers (hydrophones) are located 20 meters above the seafloor and 500 meters above the topography baseline (see figure 3.1). The row of receivers located just above the seafloor is not horizontal, but rather, it follows the topography of the seafloor.

Initially, a set of sinusoidal models with 10 meter grid spacing and a variety of amplitude and wavelength values were run. These seafloor profiles are shown in figure 3.2 and the velocity-depth function of model SIN360 for average, hill, and valley sections are shown in figure 3.3. The 'average' velocity-depth function used is the same as for the Dougherty and Stephen $(1987,1988$, chapters 1 and 2) FLAT model and is typical of young ocean crust (Ewing and Purdy, 1982; Purdy, 1982a). The effect of hills and valleys on the velocity profiles is to decrease the second gradient under hills and increase the second gradient under valleys.

The two-dimensional P-wave velocity profile for model SIN360 is shown in figure 3.4. This model has sinusoidal seafloor topography with a wavelength of 3.6 kilometers 
and amplitude of 150 meters ( 300 meters from peak to valley). Features of the results of this model demonstrate both the characteristics of scattering from large deterministic seafloor structures as well as the limitations of the finite difference grid (with coarse grid spacing) in handling the non-planar water-solid interface.

As seen in figures 3.3 and 3.4, the gradient in P-wave velocity is different between hills and valleys. In general, the first segment of the 'average' velocity-depth function follows the seafloor, that is, the vertical gradient (not the normal gradient) is the same at all points in range. The second gradient segment is deformed under the topography to be larger under valleys and smaller under hills. Also, the effective gradient for waves normally incident on the topography will be greater than the actual vertical gradient. Both of these factors will add to the travel time anomalies present in the time series.

Angle of incidence for a P-wave source at zero range is plotted against range at the seafloor for model SIN360 in figure 3.5. Also shown on the figure are the critical angles and ranges for $\mathrm{P}$ and $\mathrm{S}$-diving waves from the surface source. Critical ranges for $\mathrm{P}$ and $\mathrm{S}$ diving waves in figure 3.5 occurs where the angle of incidence curve crosses the P or Sdiving wave critical angle line. Backscattering will occur when angle of incidence is zero and ray theory 'shadow zones' would occur beyond ranges where the source path is tangent to the seafloor (angle $\mathrm{i}=\pi / 2$ ). Of course, no actual 'shadow zones' are present because of 'Franz-type' waves which are diffracted from the curved surface at grazing incidence. These diffracted waves are analogous to the circumferential waves seen in studies of acoustic plane wave scattering from large elastic cylinders (Neubauer, 1986). Multiple head waves for both compressional and shear waves will occur whenever the angle of incidence curve crosses the critical angle curve for the respective wave types then falls back below it (Stephen, 1984). 
Figure 3.1. Finite difference grid parameters for the initial rough seafloor models. The seafloor is located at a mean depth of $3.0 \mathrm{~km}$. (topography baseline). Hydrophones are located every 40 meters in range along one horizontal row in the water column and one row which follows the seafloor 20 meters above the topography. 


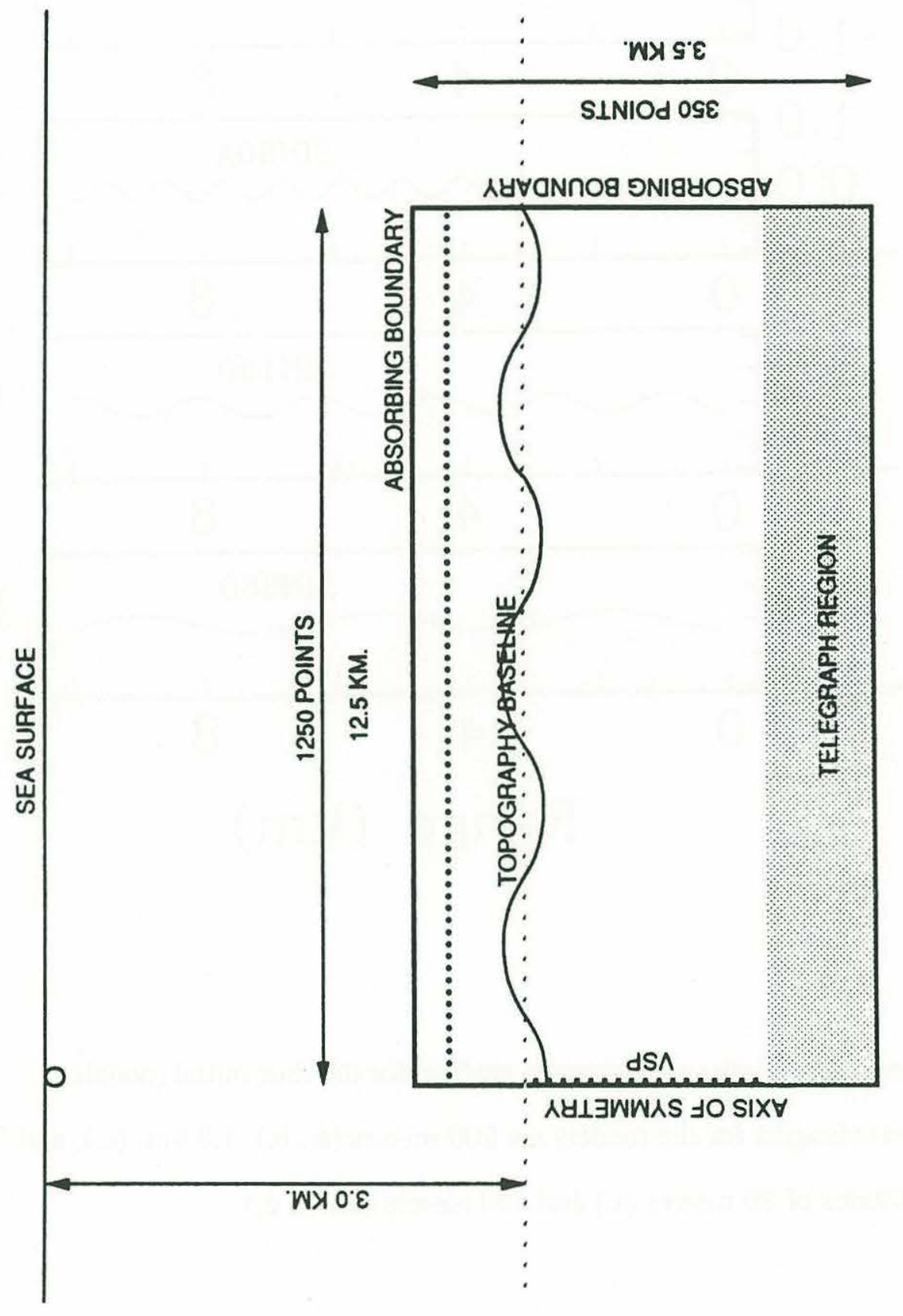




\section{Seafloor topography}

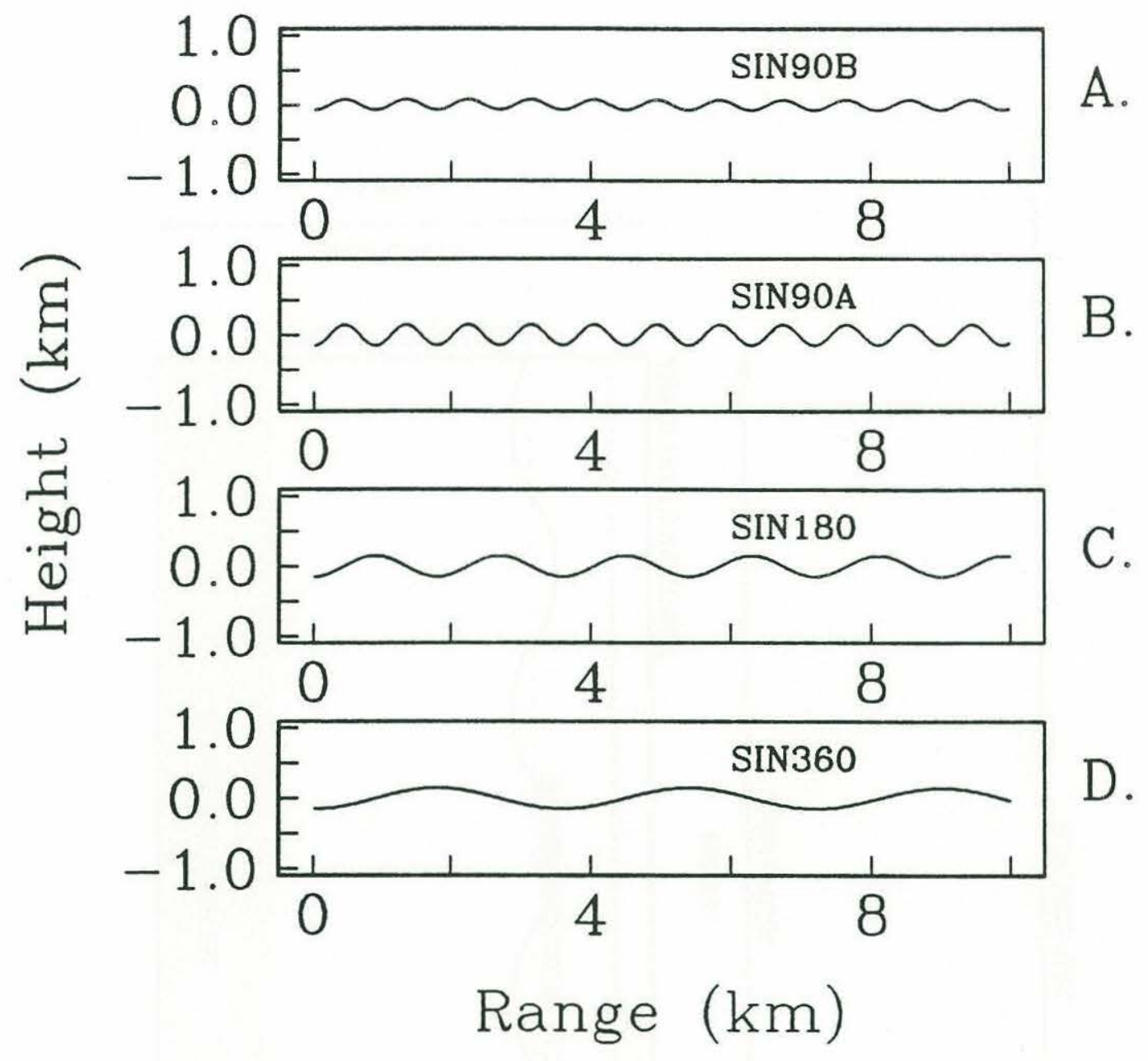

Figure 3.2. Sinusoidal seafloor topography profiles for the four initial models.

Topography wavelengths for the models are 900 meters (a., b.), $1.8 \mathrm{~km}$. (c.), and $3.6 \mathrm{~km}$. (d.) with amplitudes of 80 meters (a.) and 150 meters (b., c., d.). 


\section{$\mathrm{P}$-velocity $(\mathrm{km} / \mathrm{sec})$}

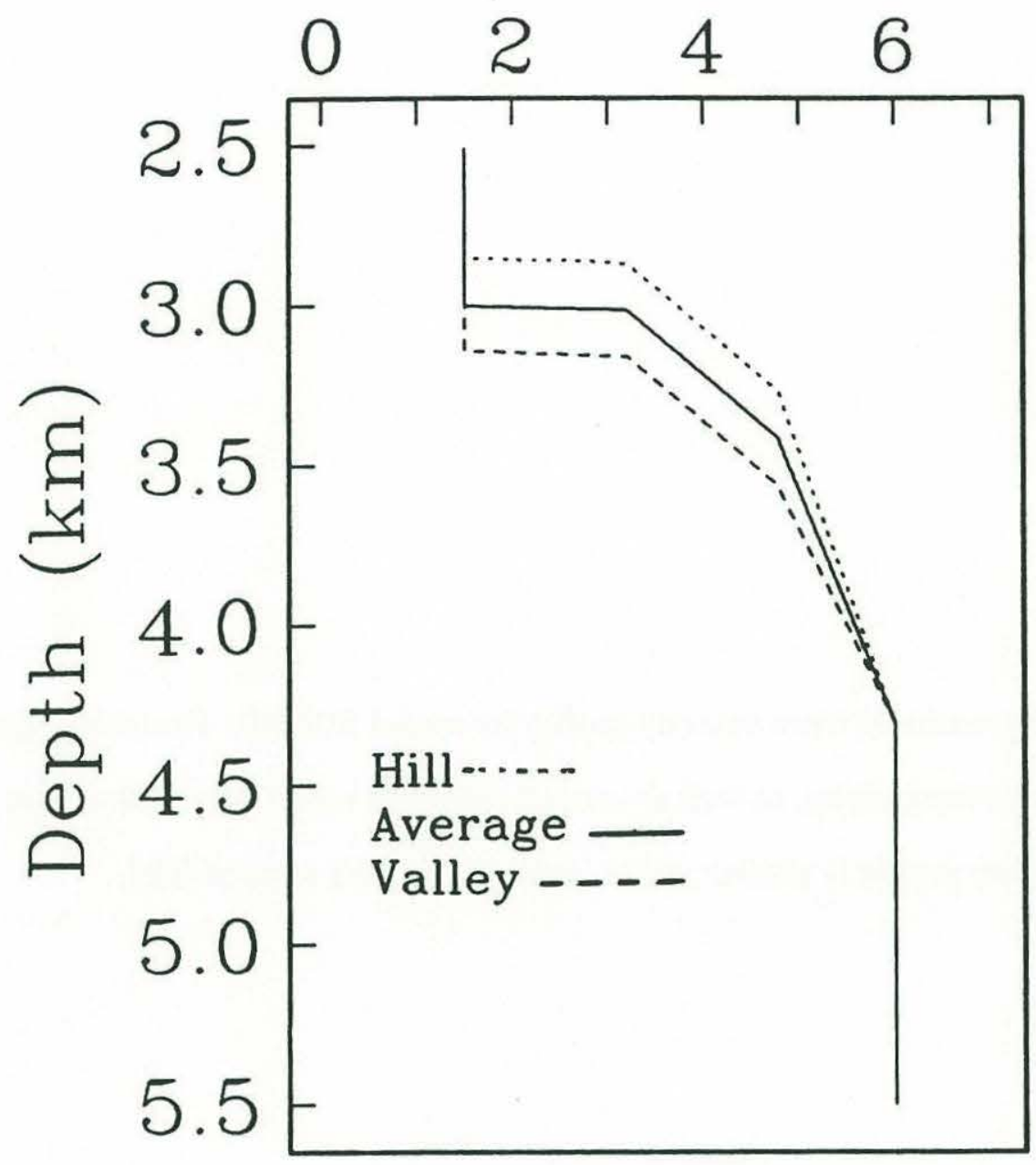

Figure 3.3. Velocity-depth functions for model SIN360 beneath hills (short dashes), valleys (long dashes) and average structure (solid line). The first gradient segment is constant regardless of structure and the second gradient segment is either lengthened or compressed to meet a constant velocity of $6.0 \mathrm{~km} / \mathrm{sec}$ at $4.3 \mathrm{~km}$. depth. Shear wave velocity-depth profiles are similar and assume a Poisson's ratio of 0.25 . 
Figure 3.4. Compressional wave velocity profile for model SIN360. Horizontal gradients are imposed by the topography, as well as vertical gradients which vary with range. The shear wave velocity profile is similar and assumes a Poisson's ratio of 0.25 . 
SIN360 P-velocity

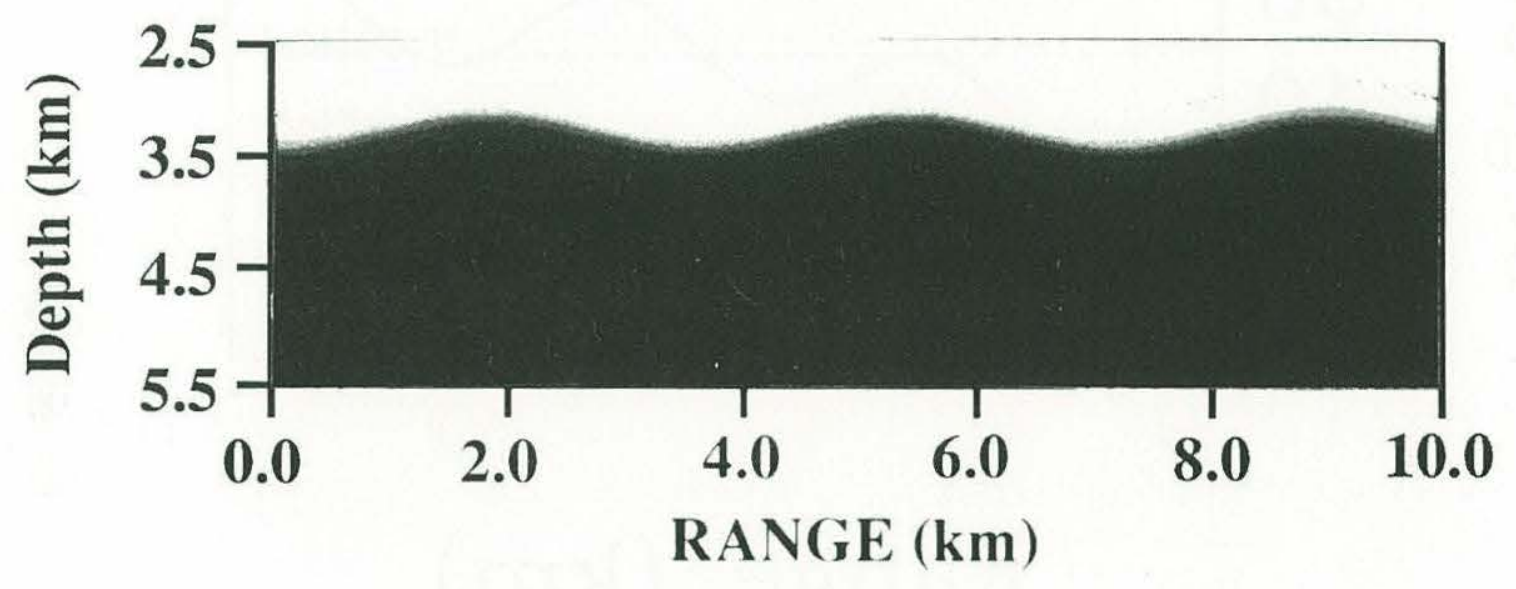




\section{Incidence angle}

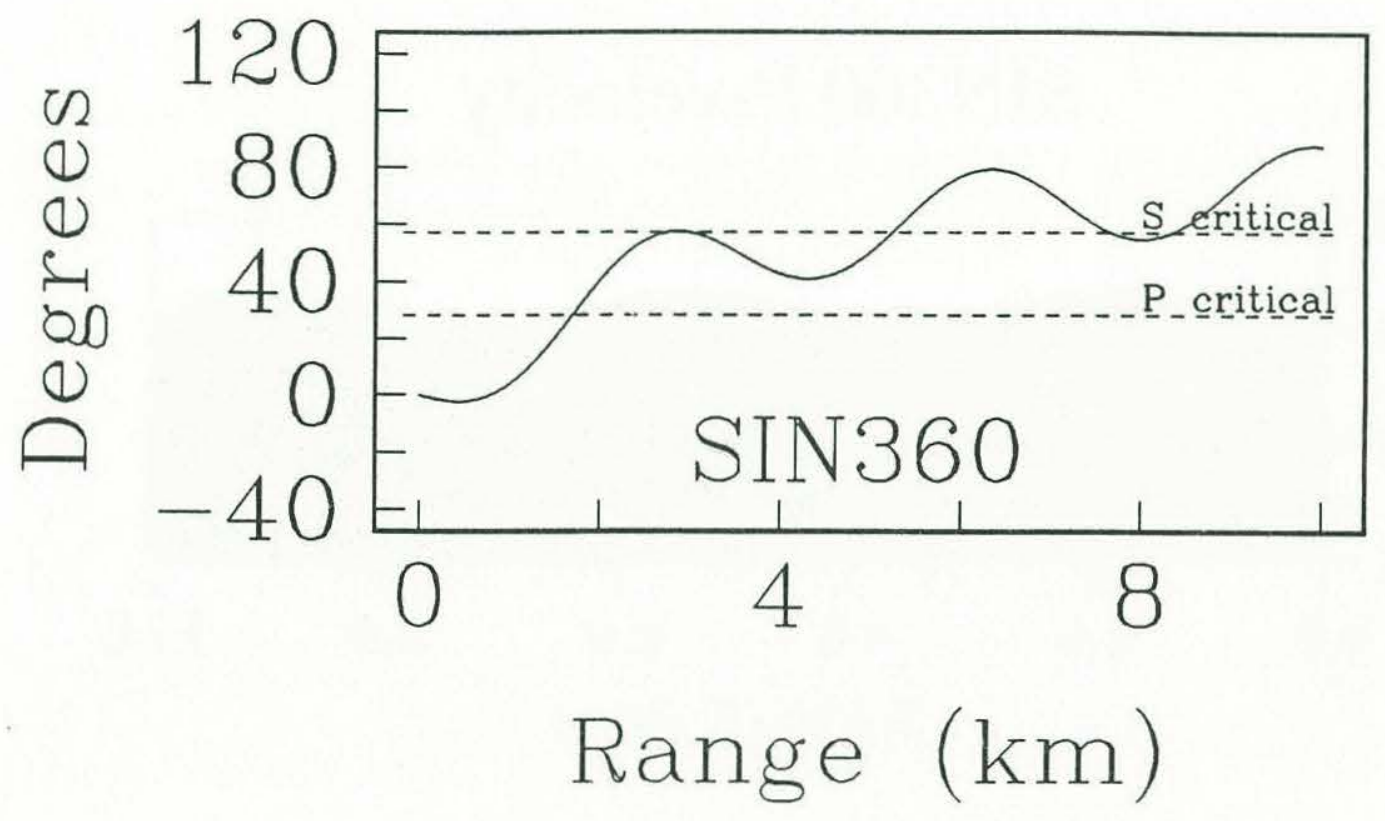

Figure 3.5. Angle of incidence vs. range for a pulse source at $0.0 \mathrm{~km}$. range and $3.0 \mathrm{~km}$. above the seafloor for model SIN360. Topography allows for multiple S-wave critical ranges. Theoretically, every range where the critical angle falls below the critical angle for $\mathrm{P}$ or S energy (given by the dashed lines), $\mathrm{P}$ or $\mathrm{S}$ energy can be directly transmitted into the bottom. 
Model SIN360, as well as models SIN90A, SIN90B, and SIN100 presented below, were run for 5000 time steps at one millisecond per time step for a total real time of 5 seconds after the surface shot (and a $10 \mathrm{~Hz}$ source). Numerical Schlieren diagrams (figure 3.6) were output every 400 time steps ( 0.4 seconds) starting at 2.0 seconds. These diagrams represent normalized compressional (top) and shear (bottom) transient energy within the model environment (chapter 2 for more on this format). Dougherty and Stephen (1988, chapter two) present both the snapshots and time series in the same format as this study for the laterally homogeneous model (FLAT) with a horizontal, planar seafloor. This laterally homogeneous FLAT model can be considered as a 'control' model for the work shown here.

Figure 3.6 is a series of snapshots which represents the time evolution of wavefronts (P-waves top and shear waves bottom) within model SIN360. The initial Pwave pulse source is just above the seafloor after 2.0 seconds (figure 3.6a). At the 2.4 second snapshot (figure 3.6b) the wavefront has partitioned into transmitted $\mathrm{P}$ and $\mathrm{S}$, reflected $\mathrm{P}$, and direct $\mathrm{P}$ waves. Note particularly the curved $\mathrm{P}$-wave front which has been reflected from the left side of the first hill. This will also show up as additional curvature in the P-diving wave arrival in the $t-x$ space of the time series. By 2.8 seconds after the shot, the wave interactions have gotten much more complex (figure 3.6c). Multiple shear and compressional transmitted waves at very close range are probably caused by constructive interference between wavefronts scattered from the stepwise curved seafloor (the curved interface is made up of a number of 10 meter rectangular steps). It will be shown later that when the grid increment is made smaller, much of this scattering will disappear.

There are a number of notable scattering features which appear in the snapshots from 3.2 to 4.8 seconds (figures 3.6d-h). Most prominent among these is the preponderance of scattered energy which is found between the primary arrivals (those seen 
Figure 3.6 a,b. Snapshots of normalized compressional (top) and shear (bottom) energy for model SIN360. The sinusoidal seafloor is located by the solid line at an average depth of $3.0 \mathrm{~km}$. 3.6a. 2.0 seconds after the shot. The cylindrical wave from the pulse line source at the sea surface is just reaching the seafloor at zero range. No shear energy is present yet in the system. $3.6 \mathrm{~b}$. 2.4 seconds after the shot. Seismic energy has interacted with the seafloor and partitioned into direct, seafloor reflected, and transmitted waves of both compressional (top frame) and shear (bottom frame) types. 


\section{SIN360}
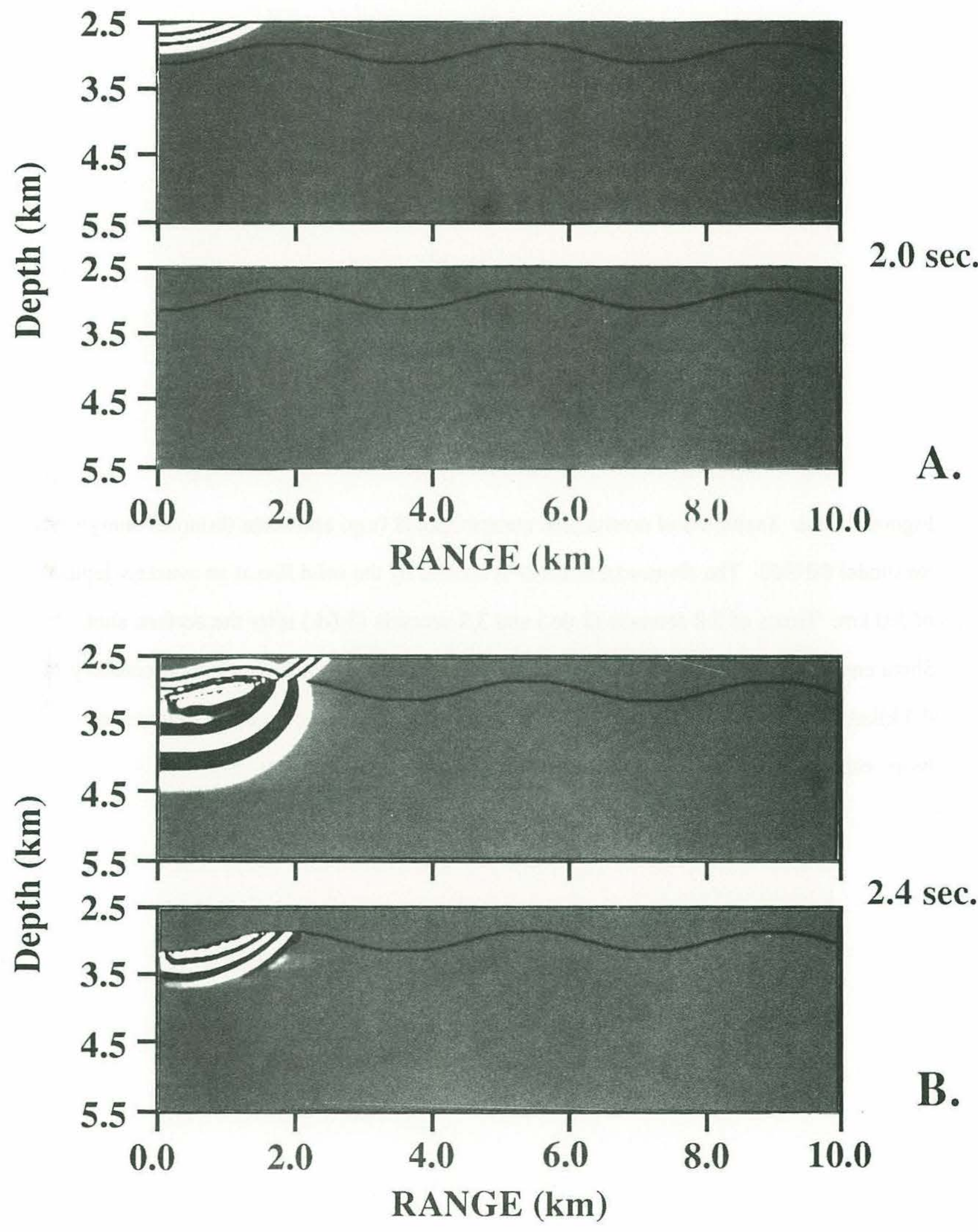
Figure $3.6 \mathrm{c}$,d. Snapshots of normalized compressional (top) and shear (bottom) energy for model SIN360. The sinusoidal seafloor is located by the solid line at an average depth of $3.0 \mathrm{~km}$. Times of 2.8 seconds (3.6c.) and 3.2 seconds (3.6d.) after the surface shot. Shear energy will continue to be transmitted into the crust out to a range of approximately 5.5 kilometers (see figure 3.5). The energy which appears random and incoherent has been scattered from the small grid-induced steps of the sloping topography. 


\section{SIN360}
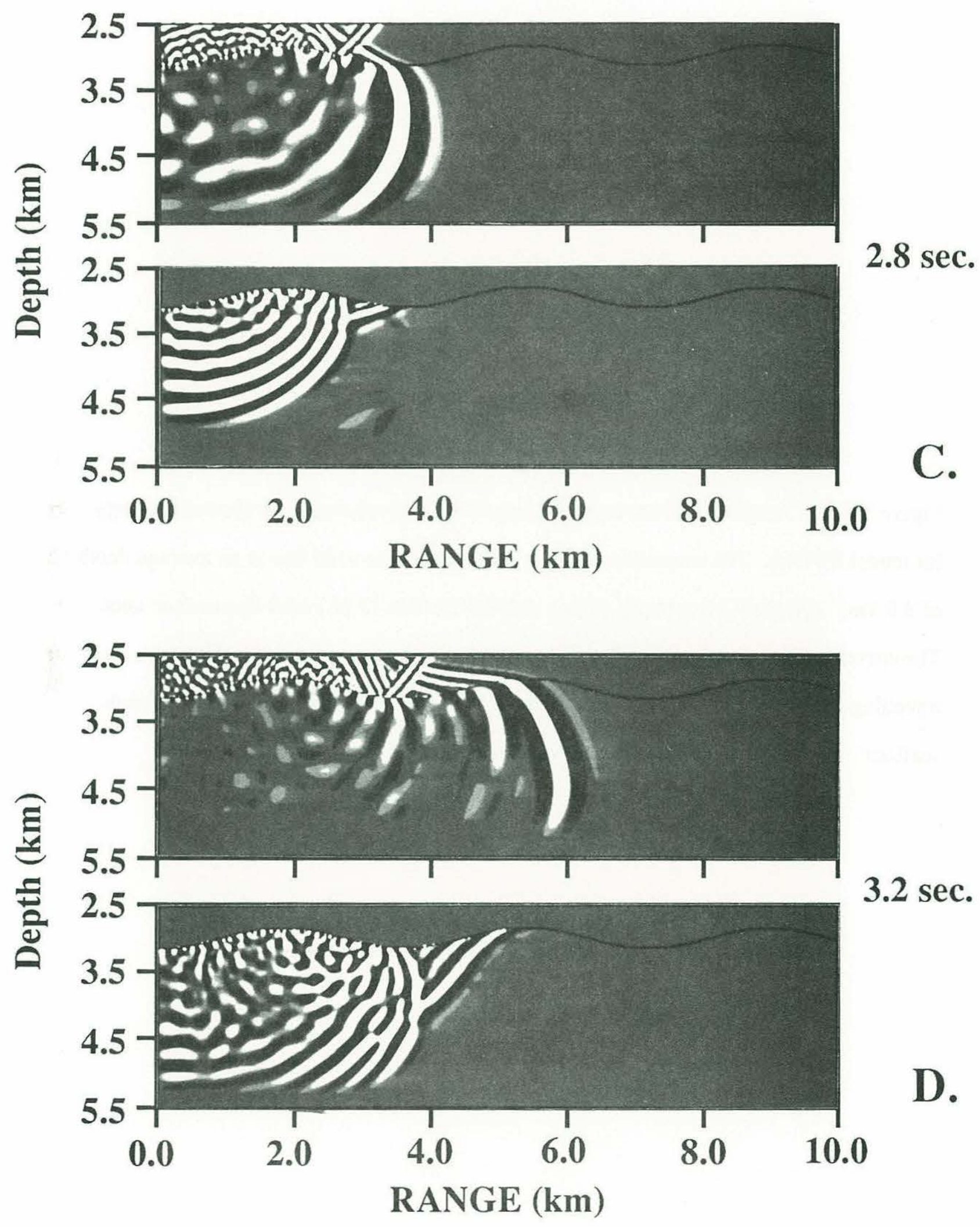
Figure 3.6 e,f. Snapshots of normalized compressional (top) and shear (bottom) energy for model SIN360. The sinusoidal seafloor is located by the solid line at an average depth of $3.0 \mathrm{~km}$. Times of 3.6 seconds (3.6e.) and 4.0 seconds (3.6f.) after the surface shot. The curved arrivals in the shear plot of 4.0 second (3.6f.) around range $2.0 \mathrm{~km}$. are travelling to the left and are shear waves which have been backscattered from the rough seafloor. These show up as curved arrivals on VSP receivers. 


\section{SIN360}

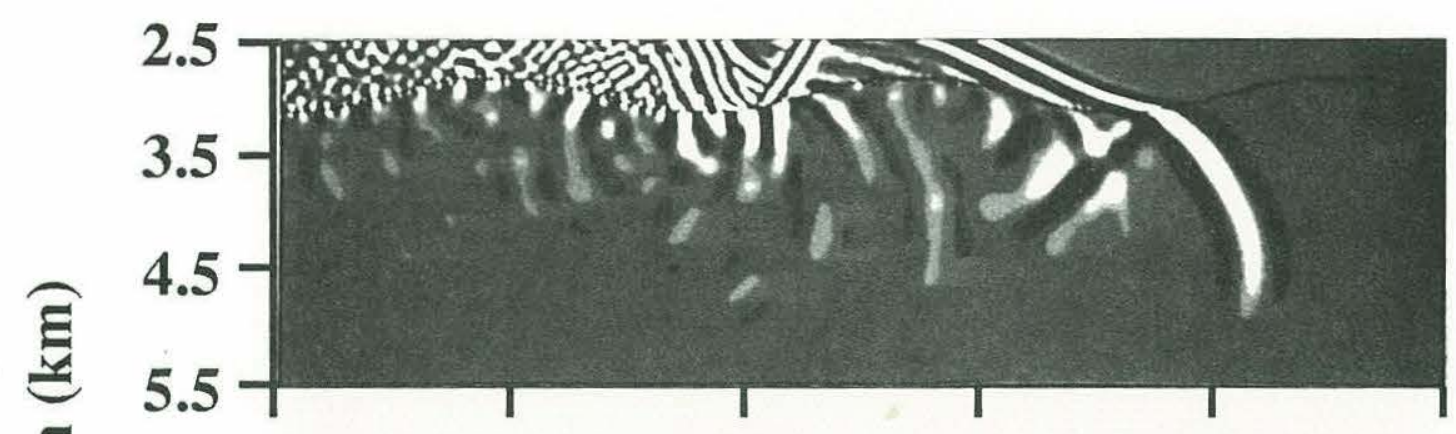

气ัँ

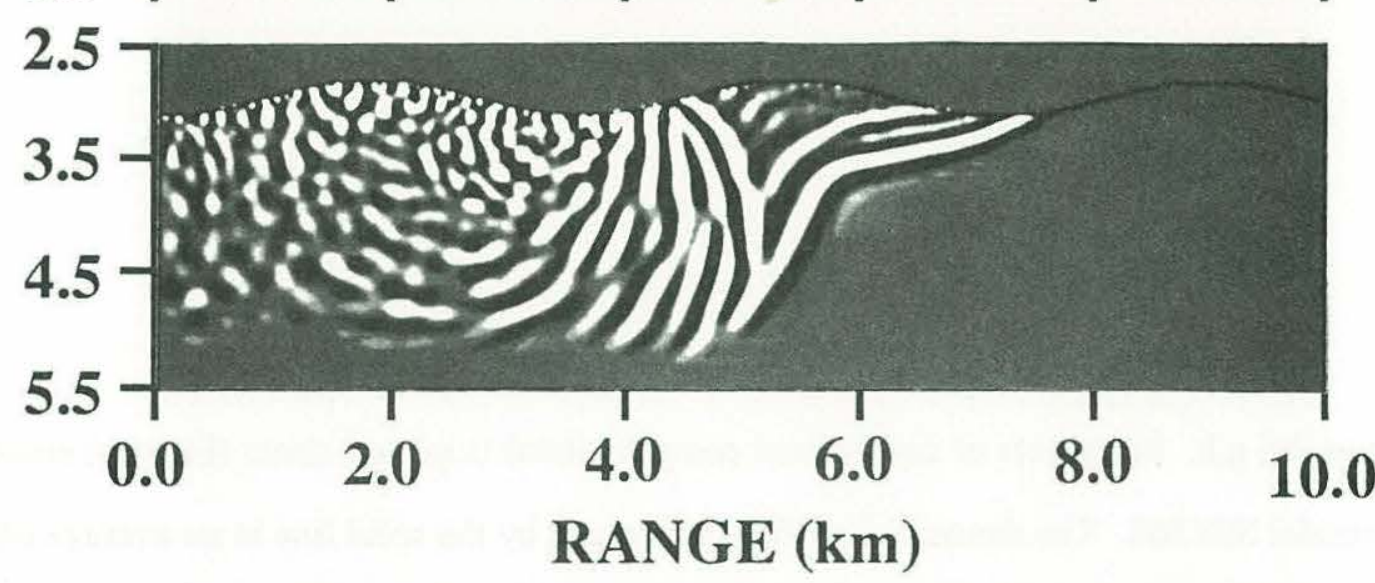

$3.6 \mathrm{sec}$.

E.

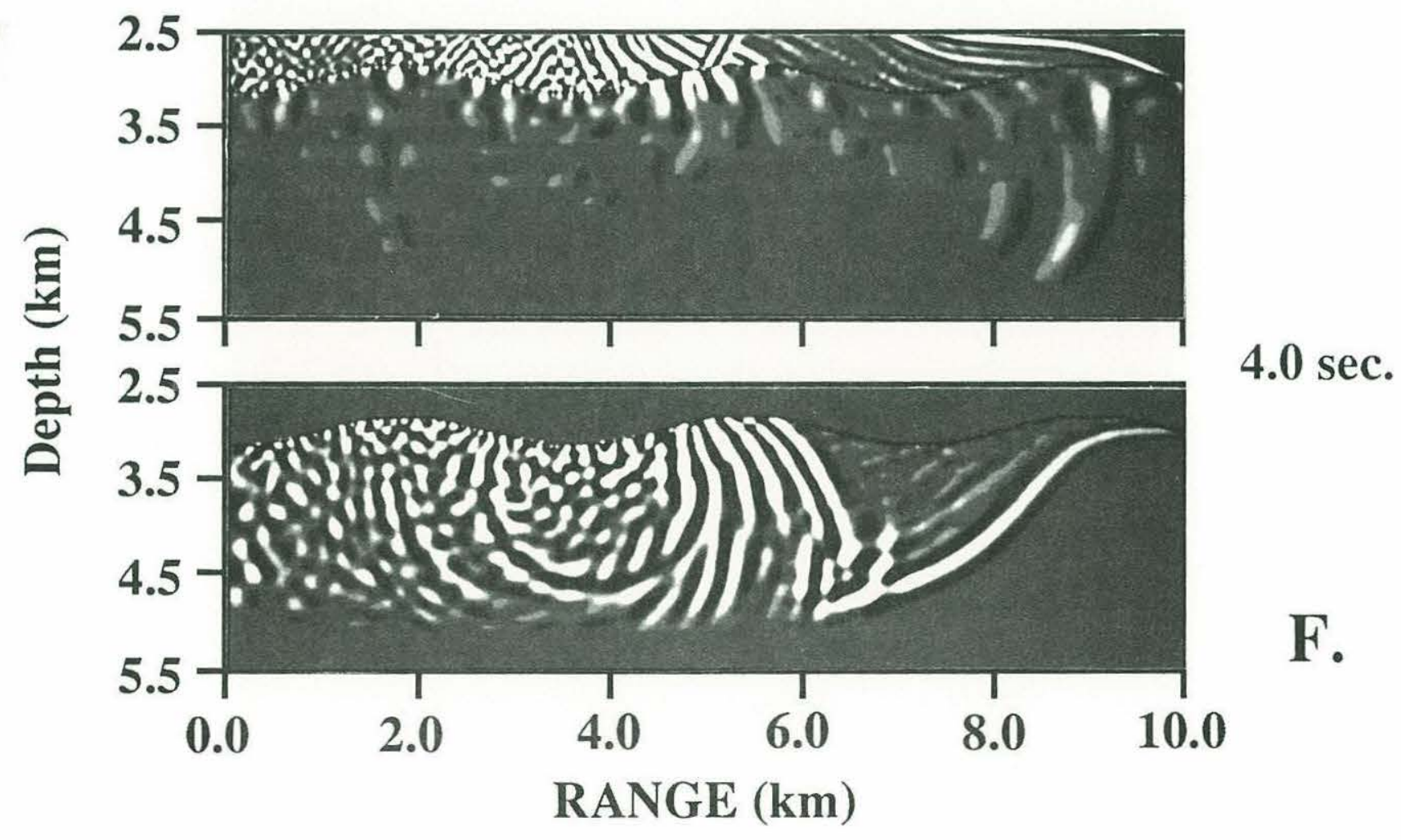


Figure $3.6 \mathrm{~g}$,h. Snapshots of normalized compressional (top) and shear (bottom) energy for model SIN360. The sinusoidal seafloor is located by the solid line at an average depth of $3.0 \mathrm{~km}$. Times of 4.4 seconds (3.6g.) and 4.8 seconds (3.6h.) after the surface shot. 


\section{SIN360}
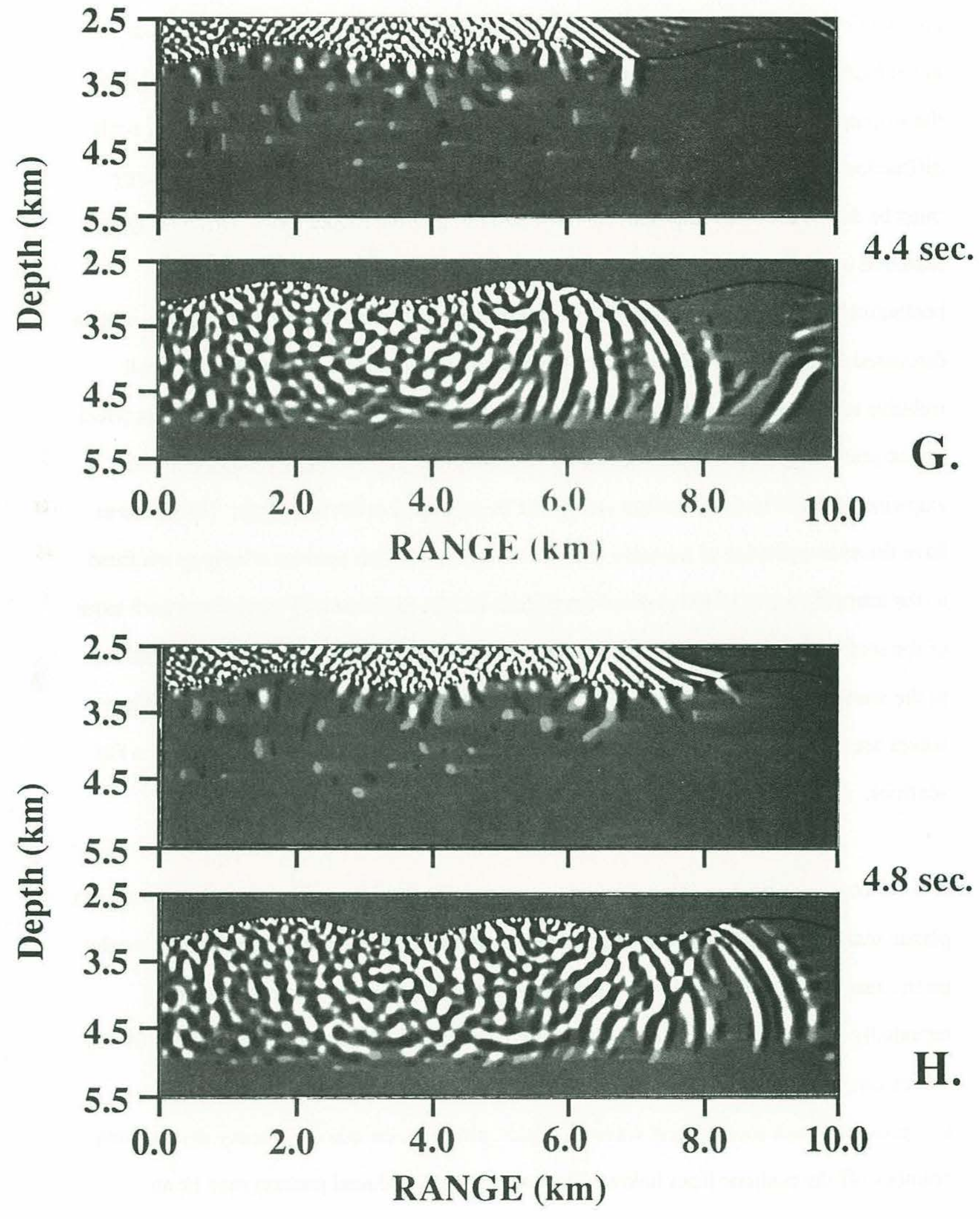
There are a number of notable scattering features which appear in the snapshots from 3.2 to 4.8 seconds (figures $3.6 \mathrm{~d}-\mathrm{h}$ ). Most prominent among these is the preponderance of scattered energy which is found between the primary arrivals (those seen in the FLAT model of chapters one and two plus additional diving waves associated with the sloping sea floor). Since the slopes in model SIN360 are relatively small, small scale diffraction type scattering is not expected. Therefore, the incoherently scattered energy must be due to the steps imposed by coarse sampling of the topography. This energy is scattered in all directions from the seafloor. Also, a significant amount of energy is backscattered or reverberated into the water column. The accuracy of this scattering will be discussed in more detail below, but for these preliminary models, it appears that small (relative to the seismic wavelength) sharp topographic features can have a significant effect on the scattering of seismo/acoustic energy. One consequence of this scattering at the stepwise interface is that interface waves can be generated at the boundary. These waves have the characteristics of Stoneley waves and appear as small packets of energy confined to the immediate area of the seafloor (amplitude decays exponentially away from both sides of the seafloor) and they travel at a velocity just less than the compressional wave velocity in the water (Luppé and Doucet, 1988; Scholte, 1947; Stoneley, 1924). These interface waves are similar to those generated by scattering from volume heterogeneities with a flat seafloor.

Compressional and shear diving waves generated from an acoustic pulse source in a planar seafloor environment are singular and continuous. However, when the topography on the seafloor is steep enough to cause the angle of incidence of the direct wave to repeatedly fall below the critical angle for diving energy, additional distinct $\mathrm{P}$ and S-diving waves can occur. These additional diving waves must not be confused with compressional and shear diving wave multiples which are caused by primary diving wave bounces off the seafloor from below. This critical angle induced process may be an 
of incidence to fall below the critical angle for P-waves after the primary P-wave (see figure 3.5). There is, however, a relatively strong P-wave multiple that can be seen in the 3.2 second snapshot (figure 3.6d) which, along with the interference P-headwave (made up of a superposition of all P-wave multiples) rapidly decreases in amplitude in succeeding snapshots. The shear wave critical angle is much higher than that for compressional waves and the topography of SIN360 is steep enough to allow for an additional S-diving wave. This diving wave falls directly behind the primary S-diving wave in the 4.0 second snapshot.

A more traditional means of observing the synthetic seismic data is by the use of time series of hydrophone response. Hydrophone receivers were placed in 2 rows, one located 20 meters above the seafloor and one horizontal array located 500 meters above the topography baseline (the point in the sine curve where amplitude is zero) as seen in figure 3.1. Hydrophones were spaced every 8 grid points or 80 meters apart in both receiver rows. The time series from both rows of receivers of model SIN360 are shown in figure 3.7. Two amplitude scales are presented for each seismogram. The smaller amplitude scale was used to display an unclipped direct/reflected wave and a scale factor five times larger was used to display a visible and unclipped P-diving wave. None of the seismogram arrivals have been corrected for topography (as in Purdy, 1982b) so that in general the arrivals (except for the direct arrival) for the horizontal row of receivers will appear more curved than those for the seafloor hydrophones.

Figures $3.7 \mathrm{c}$ and $3.7 \mathrm{~d}$ are the pressure time series for the row of hydrophones along the seafloor plotted at the two different amplitude scales. The P-diving wave is only slightly curved due to travel time anomalies induced by the different velocity-depth profiles below hills and valleys. Amplitude anomalies due to focussing/defocussing around the topography are prominent along the entire range of the arrival when compared to the FLAT 
Figure 3.7 a,b. Waterborne hydrophone receivers for model SIN360. 3.7a. Arrivals have been scaled to show an unclipped direct wave. Discontinuity of the reflected arrival is caused by multiple shear wave critical ranges caused by the sinusoidal topography (see figure 5). 3.7b. Amplitudes are increased by a scale factor of five over figure 3.7a. to show the unclipped P-diving wave. Scattered arrivals after the reflected and P-diving waves are quite significant with respect to the $\mathrm{P}$ and $\mathrm{S}$ primary transmitted waves. 

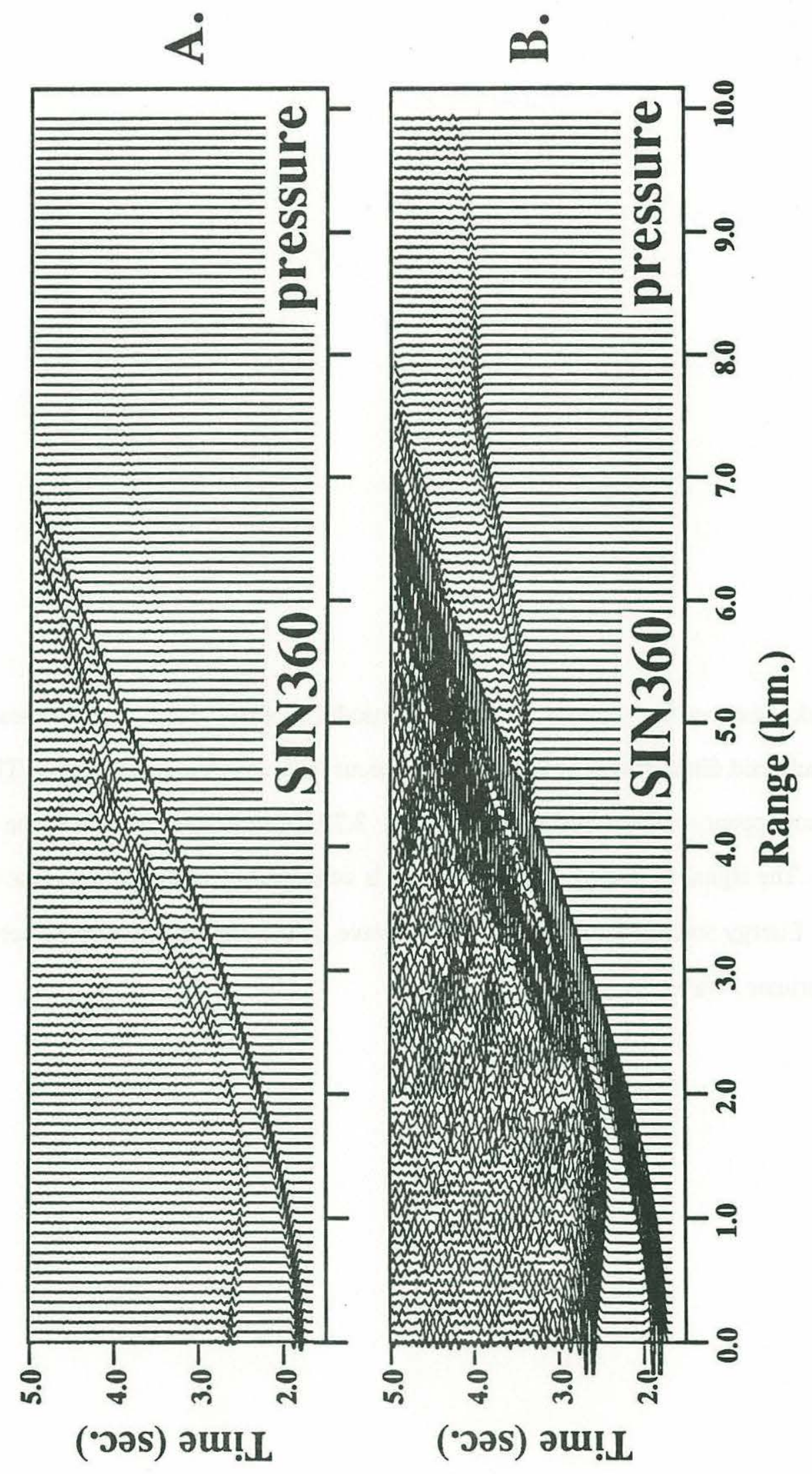
Figure $3.7 \mathrm{c}$,d. Seafloor hydrophone receivers for model SIN360. 3.7c. Amplitudes relative to unclipped direct wave. Interface waves occur following the direct wave. This 'noise' does not appear on the waterborne receivers. 3.7d. Amplitudes relative to the Pdiving wave. The signal following the direct wave is swamped by scattered interface and body waves. Energy scattered out of the P-diving wave by the topography arrives between the $\mathrm{P}$ and $\mathrm{S}$ primary waves. 

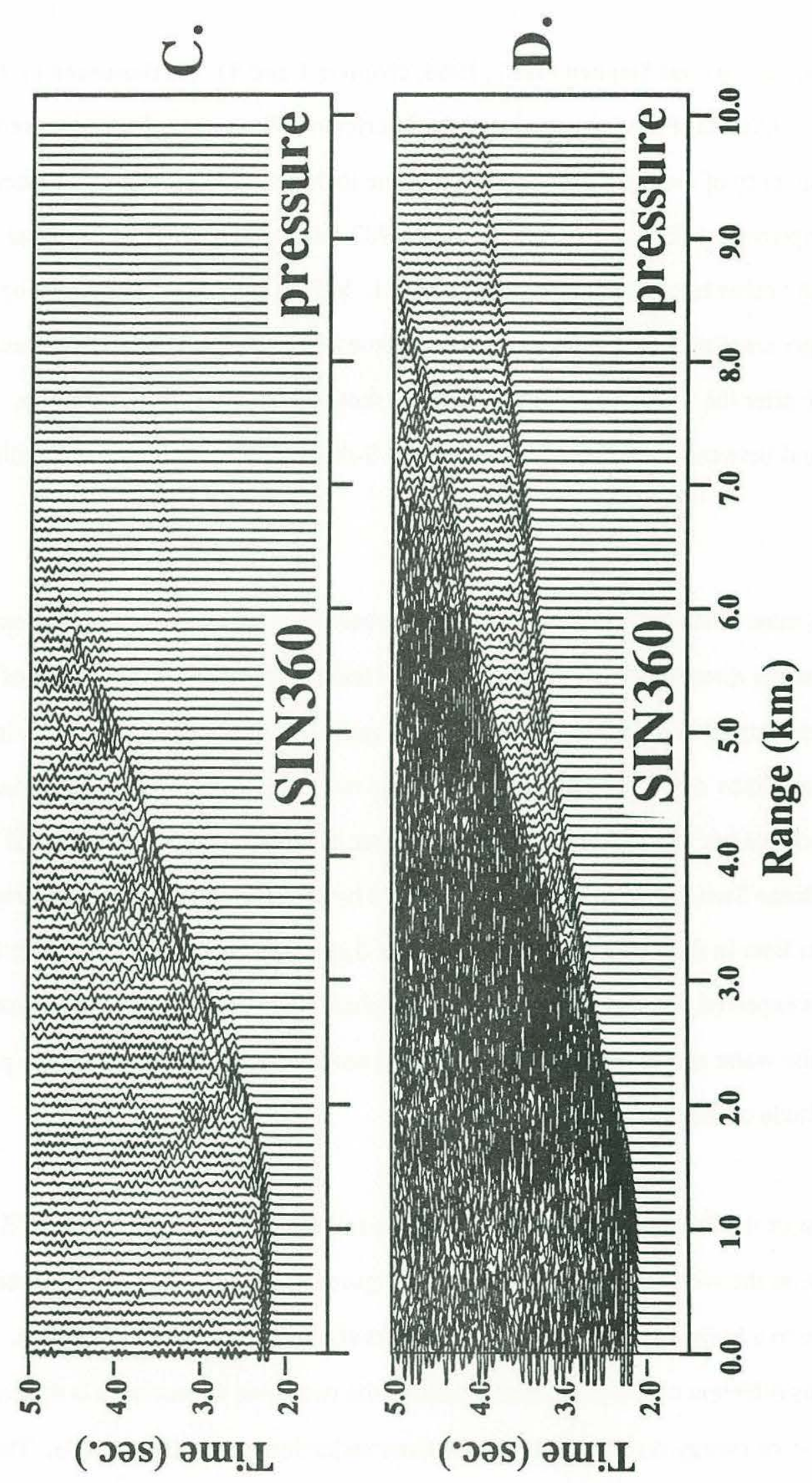
model of Dougherty and Stephen (1987, 1988, chapters 1 and 2). As discussed earlier, there are no additional P-diving waves and the interference $\mathrm{P}$-wave is also not present. The lack of occurrence of the interference P-wave is due to destructive interference caused by the rough topography (Dougherty and Stephen, 1987 (chapter 1)). Additional shear waves, on the other hand, do appear in figure $3.7 \mathrm{~d}$. In fact, the second shear-diving wave has a stronger amplitude than the first arrival at some ranges. Scattered shear waves are present both after the direct wave (although these show up better in the waterborne receivers) and between the P-diving wave and the S-diving waves (primary and additional arrivals).

The most obvious feature of the seafloor receivers is the amount of strong energy arriving after the direct wave for ranges between 0 and 6 kilometers. While some of this energy is made up of scattered body waves, the majority of the energy is travelling in the form of an interface wave. The Stoneley waves are travelling in both the forward and reverse directions but are difficult to distinguish from the direct wave in the forward direction. Some Stoneley waves are also generated by scattering of the P-diving wave and show up just later in time than the P-diving arrival (i.e. at approximately $7.5 \mathrm{~km}$. in figure 3.7d). As is expected, the Stoneley waves have a phase velocity less than the acoustic velocity in the water and in general have very large amplitude, comparable in some places to the amplitude of the direct compressional wave.

Most of the features present in the seafloor seismograms (figures 3.7c and 3.7d) are also present on the waterborne receivers seen in figures 3.7a and 3.7b. Again, these receivers are in a horizontal row located 500 meters above the topography baseline. The most obvious difference between seismograms of the two rows of receivers is the relative lack of 'noise' or energy arriving after the seafloor reflection of the direct wave. This energy in the seafloor time series is the Stoneley wave energy which decreases 
exponentially in amplitude away from the seafloor. The row of receivers placed 500 meters above the seafloor does not pick up this energy confined to the seafloor. As a result, the backscattered body waves, mainly of S-type, show up as distinct arrivals after the reflected wave in the upper row of receivers. In general, however, the amount of backscattered energy is much less in these receivers than for those along the seafloor. The greater curvature of the P-diving and reflected waves is due to variable water path lengths caused by the topography. If travel times were explicitly being investigated, this could be corrected in part by applying a topography correction to the data.

Another, way to observe body waves within the crust is the vertical seismic profile (VSP). A VSP is carried out by recording normally incident shots at a number of locations in a borehole or along a vertical array. When the source and receiver array are offset from normal incidence, this type of experiment is referred to as an oblique seismic experiment (OSE, Stephen, 1983; Stephen et al., 1979; Stephen., 1980; Stephen, 1984). By using the finite difference method, we have the ability to place receivers anywhere on the grid. SIN360 was rerun with a vertical arrays of geophones starting at zero range and spaced every kilometer out to the boundary of the model. Scattered body waves will have a similar appearance in both the VSP and OSE geometries. The purpose here for looking at a vertical array is not to obtain velocity information, but rather, to observe sideways travelling waves (specifically backscattered body waves).

The vertical array of geophones can be seen in figure 3.1 and a cartoon of 'expected' P-wave arrivals for a laterally homogeneous environment is shown in figure 3.8. A reflected arrival from a layer within the depth of the vertical array would intersect one of the primary arrivals at the depth of the layer. A near vertical reflection for a deeper layer would have an arrival shape much like that of a sideways travelling (backscattered) body wave, but there are no reflectors below the seafloor in this model. S-wave arrivals 
Figure 3.8. Arrivals expected at a vertical array of geophones. 


\section{Depth}

옹

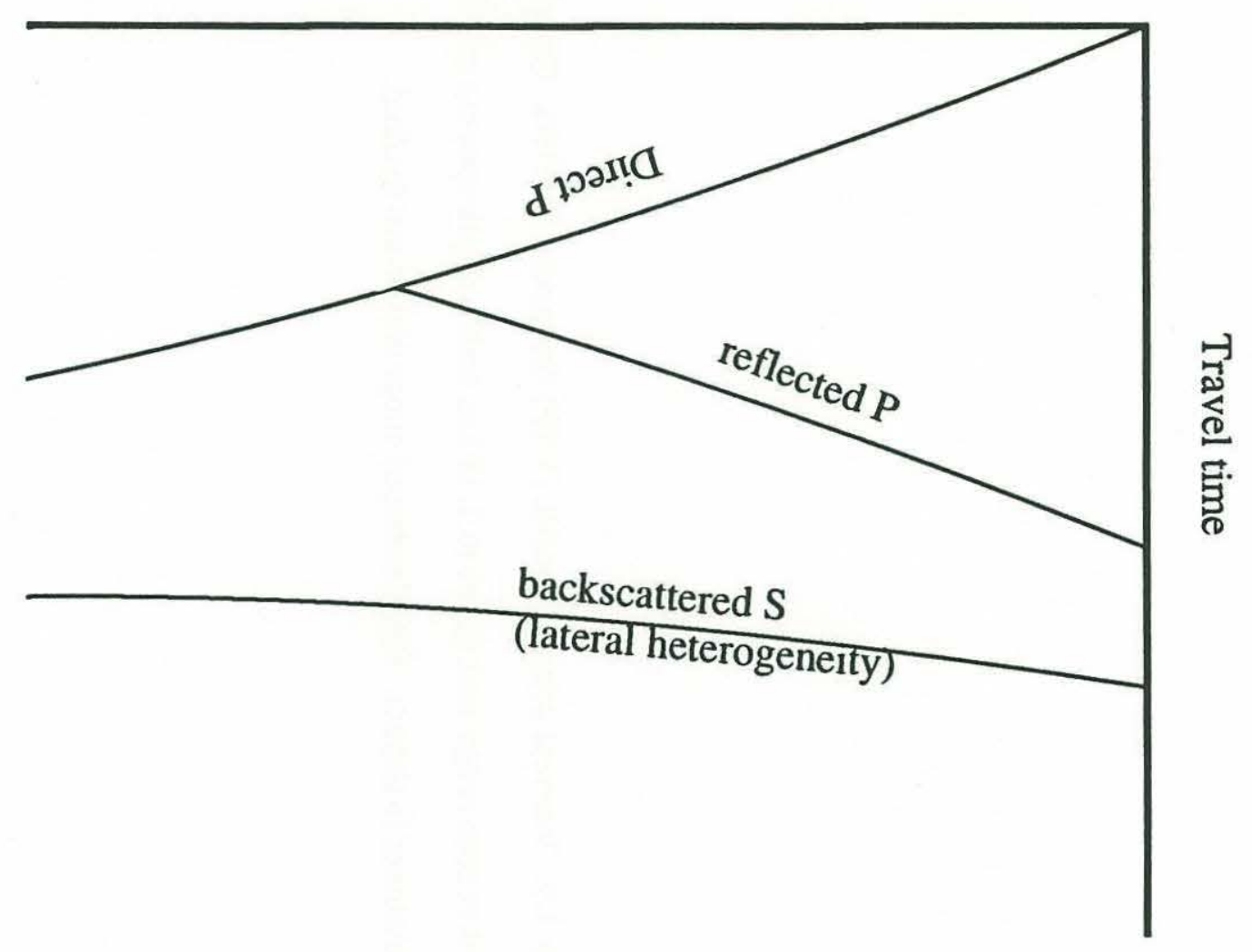


Figure 3.9. Vertical seismic profile (VSP) for model SIN360. Geophone receivers are located at zero range and down to $1.48 \mathrm{~km}$ with a depth spacing of 40 meters. Vertical displacement is shown. Backscattered shear waves are marked. 


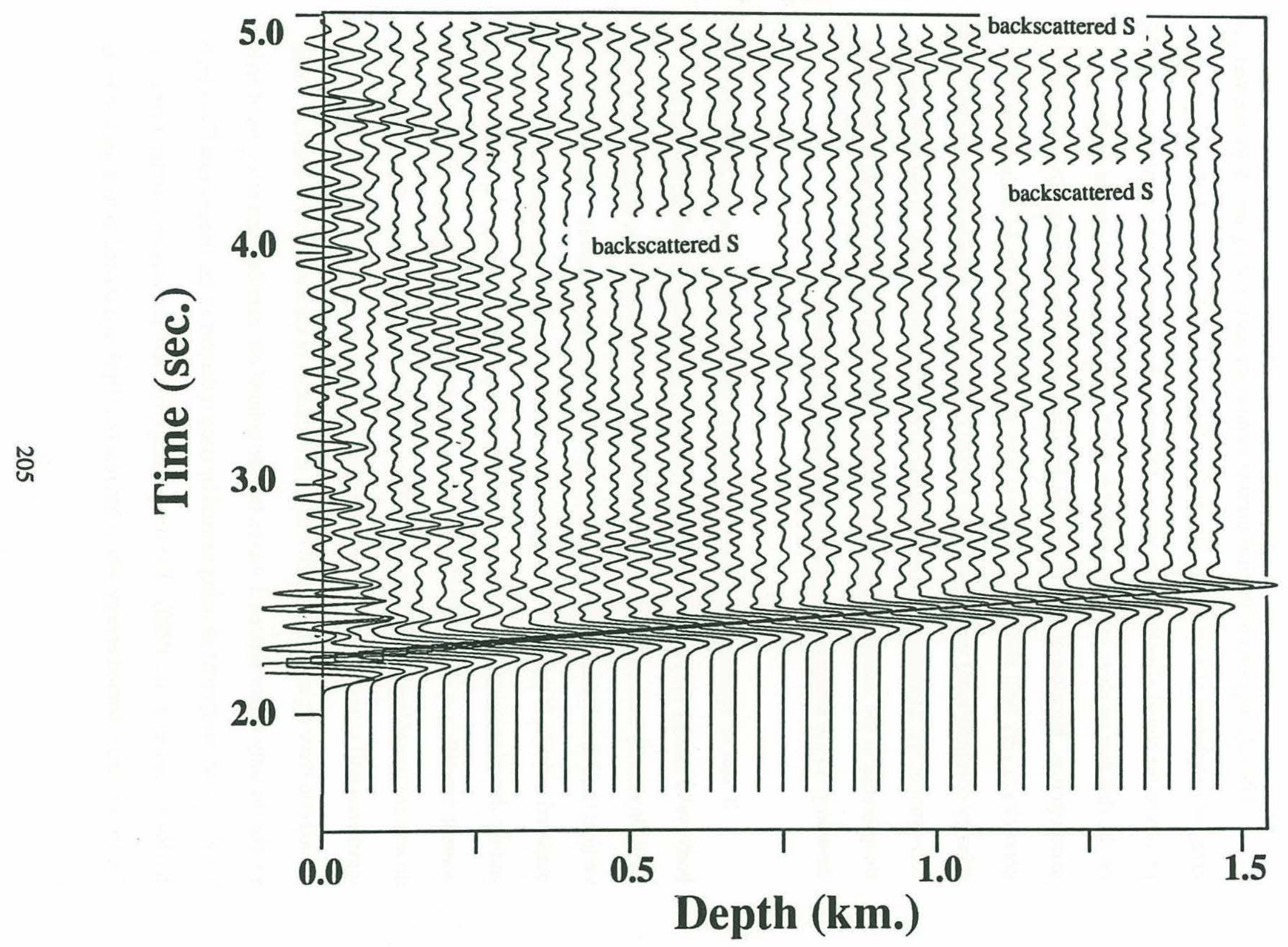


would be similar but with a different particle motion than P-wave arrivals.

The seismograms of vertical particle motion are shown in figure 3.9 for the vertical array of geophones at zero range. At shallow depths, the high amplitude Stoneley waves dominate the signal after the direct wave. However, the interface waves only have an effect on the first few receiver locations so that at depth, only body waves appear on the seismograms. Because of the fact that the interface waves die off so rapidly, the vertical geometry is very well suited for looking at both primary and scattered body waves. The primary compressional wave is the first arrival on both plots. Primary shear waves are not converted at the interface for normal incidence compressional energy. Since there are no sharp breaks in the velocity profile of the model (other than the seafloor) all of the rest of the energy in the seismograms must be scattered body waves.

It can be assumed from the results seen in the snapshots that most of the scattered body wave energy is in the form of shear waves. Since a large part of the energy is travelling nearly horizontally (at least at later times) the relatively large amplitudes in the vertical motion seismogram would also indicate scattered shear waves. The shapes of the scattered arrivals, if they can be singled out, are indicative of the curvature of the wavefront and the direction (relative to horizontal) it is travelling. Simplistically, if a scattered plane wave is travelling upwards, it will have a negative slope in the t-x space of figure 3.9. A downward travelling wave will have positive slope in the seismogram. Curvature of the wavefront will cause a slight curvature in the seismogram. Arrivals in figure 3.9 which are curved and have a generally negative slope are marked as backscattered S-waves. These are not the only backscattered S-waves but are pointed out here because this type of arrival has also been interpreted as being caused by deep reflectors in the ocean crust (Becker, et al., 1988; Kennet et.al., 1980). The unique shape of these arrivals on a vertical array is due to the backscattered energy which has reached depth and started to turn back towards 
the seafloor. The relatively high velocities at depth causes the arrival times at depth to be earlier than the arrival times near the water-solid interface. Since these wavefronts can also be clearly seen in the snapshots, it is our contention that these arrivals seen in vertical seismic arrays are not due to near vertically travelling $\mathrm{P}$-waves, but rather, from near horizontally travelling shear waves which have turned at depth.

Three other initial models were run to study the general effects of the influence of sinusoid wavelength and amplitude on the scattering of seismic energy. The seafloor profiles of these models are shown in figure 3.2 and have wavelengths of 900 meters and 1.8 kilometers. Two of the models, SIN90A and SIN90B have the same wavelength but different amplitudes ( 150 meters and 80 meters respectively). The different seafloor topographic profiles are manifest in two ways. First, the angle of incidence vs. range plots for the three models (figure 3.10) show many more critical angle crossings than for SIN360 which has a much larger wavelength. Sharper topography, especially in model SIN90A causes much more complicated horizontal gradients to be present just below the seafloor.

Results for model SIN180 are shown in figure 3.11. Many of the same features seen in SIN360 are also present in this model. Additional diving waves of both compressional and shear types are present in SIN180 and can be seen in both the 4.0 second snapshot and time series plots. Additional P-waves seen in the time series were not present in SIN360 and are due to the slightly more variable slopes present with the shorter wavelength topography. Also, many more additional S-waves are possible (seefigures $3.7 \mathrm{a}$ and $3.7 \mathrm{~b}$ ) because of the steeper, shorter hills. In general. more energy is able to enter the crust at large ranges as shear energy than in SIN360 because sections of topography with angles of incidence less than the critical angle are present even at large ranges (see figures 3.10 and 3.11). Of course, steeper, shorter topography also produces 
Figure 3.10. Angle of incidence vs. range for models SIN90B (3.10a.) SIN90A. (3.10b.) and SIN180 (3.10c.). Wavelength of the topography is 90 meters for SIN90A and SIN90B and $1.8 \mathrm{~km}$. for SIN180. Amplitude for the topography is 150 meters for SIN90B and SIN180 and 80 meters for SIN90A. 


\section{Incidence angle}

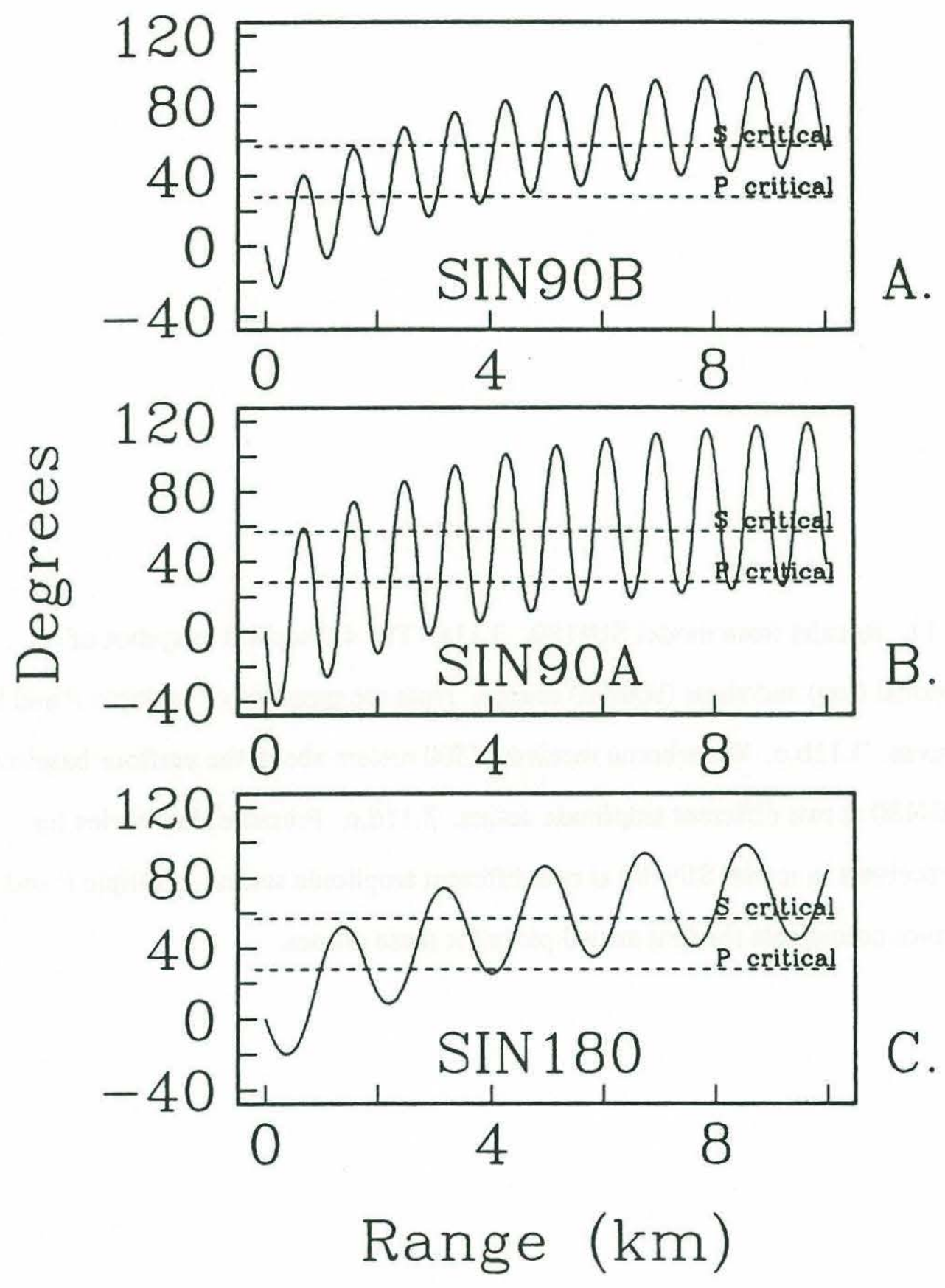


Figure 3.11. Results from model SIN180. 3.11a. The 4.0 second snapshot of compressional (top) and shear (bottom) energy. Note the presence of multiple P and S diving waves. 3.11 b,c. Waterborne receivers ( 500 meters above the seafloor baseline) for model SIN180 at two different amplitude scales. 3.11d,e. Pressure time series for seafloor receivers in model SIN180 at two different amplitude scales. Multiple P and S diving waves complicate the first arrival picks for these phases. 


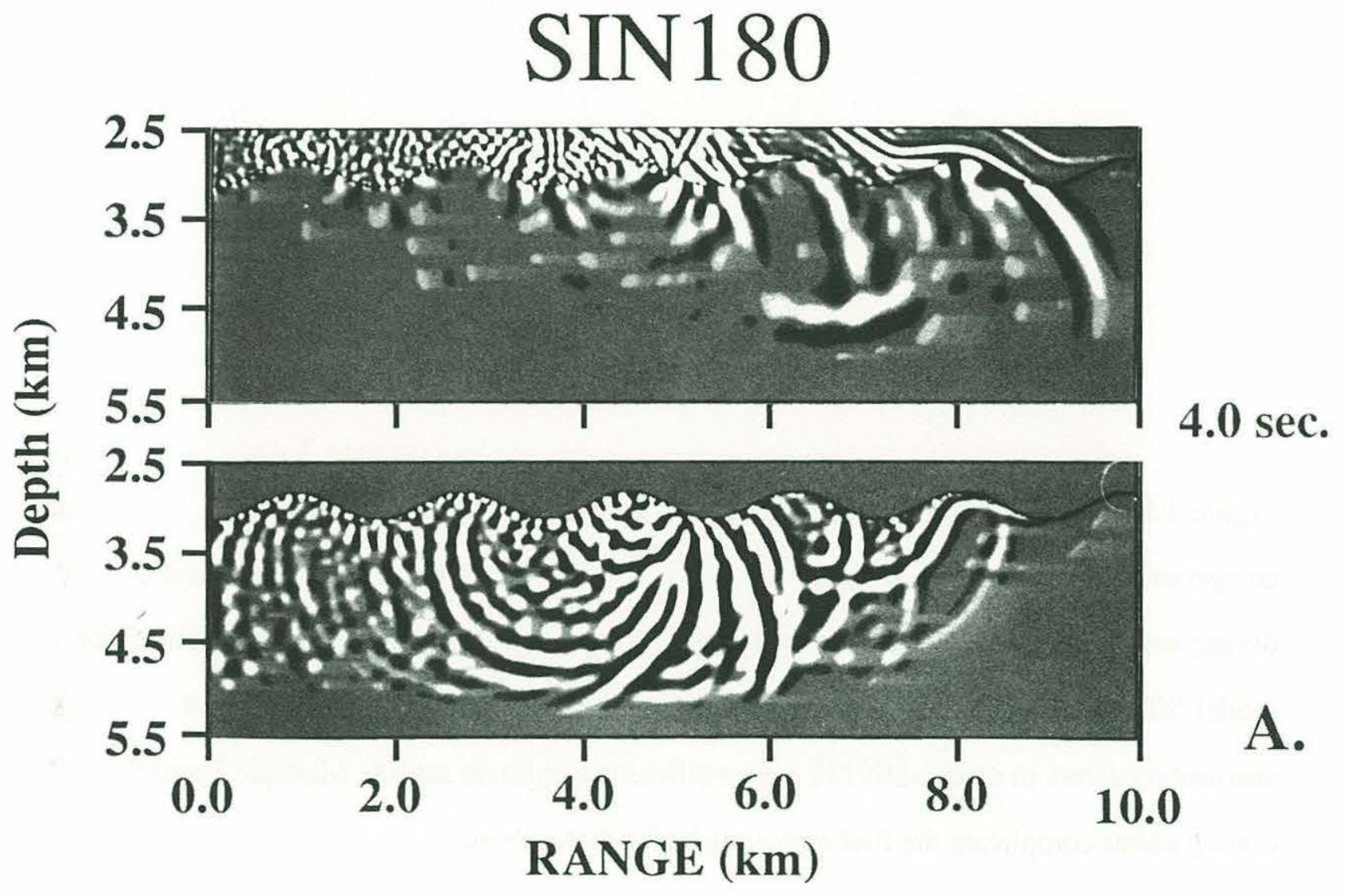


Figure 3.11. Results from model SIN180. 3.11a. The 4.0 second snapshot of compressional (top) and shear (bottom) energy. Note the presence of multiple $\mathrm{P}$ and $\mathrm{S}$ diving waves. 3.11 b,c. Waterborne receivers ( 500 meters above the seafloor baseline) for model SIN180 at two different amplitude scales. 3.11d,e. Pressure time series for seafloor receivers in model SIN180 at two different amplitude scales. Multiple P and S diving waves complicate the first arrival picks for these phases. 

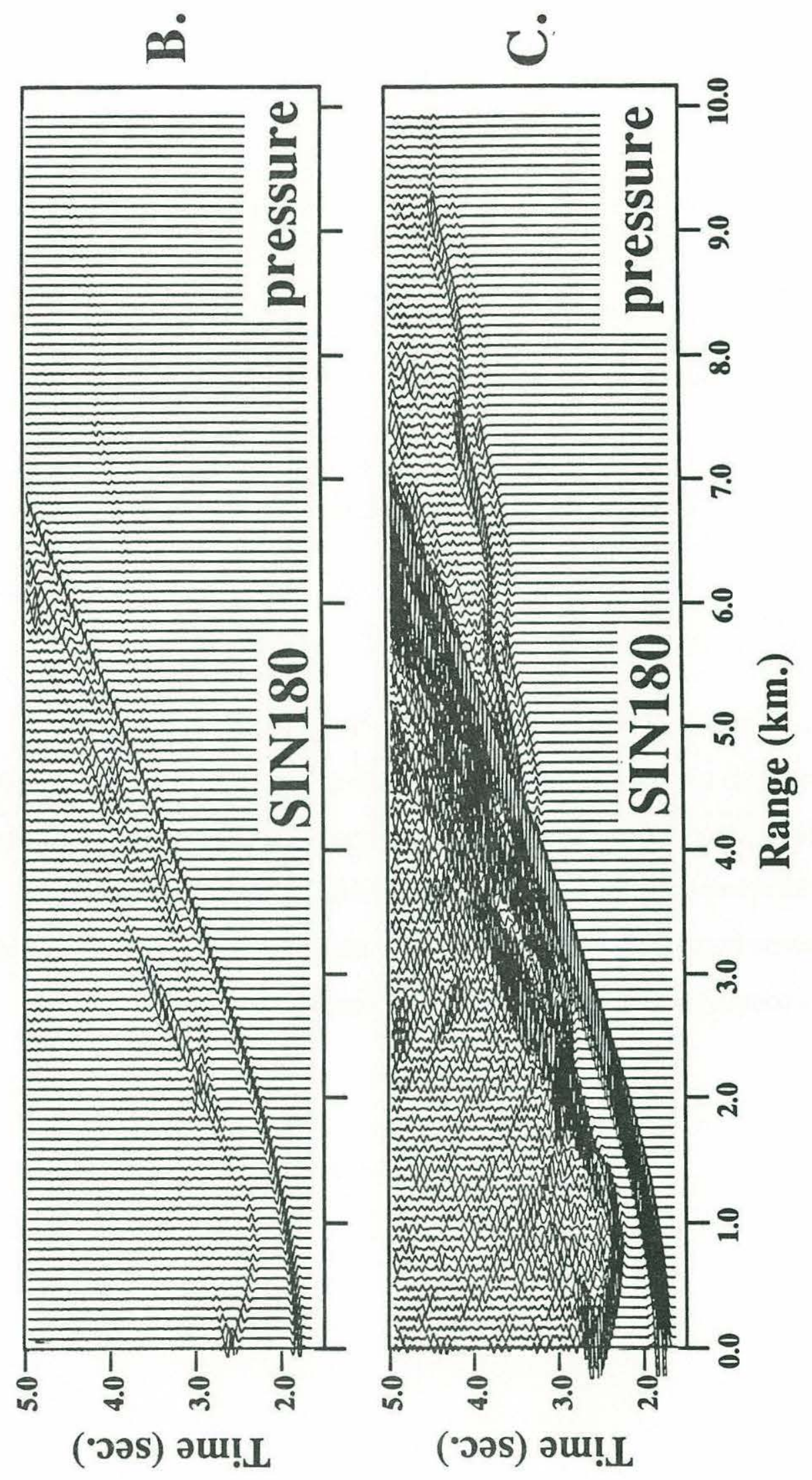
Figure 3.11. Results from model SIN180. 3.11a. The 4.0 second snapshot of compressional (top) and shear (bottom) energy. Note the presence of multiple P and S diving waves. 3.11 b,c. Waterborne receivers ( 500 meters above the seafloor baseline) for model SIN180 at two different amplitude scales. 3.11d,e. Pressure time series for seafloor receivers in model SIN180 at two different amplitude scales. Multiple P and S diving waves complicate the first arrival picks for these phases. 


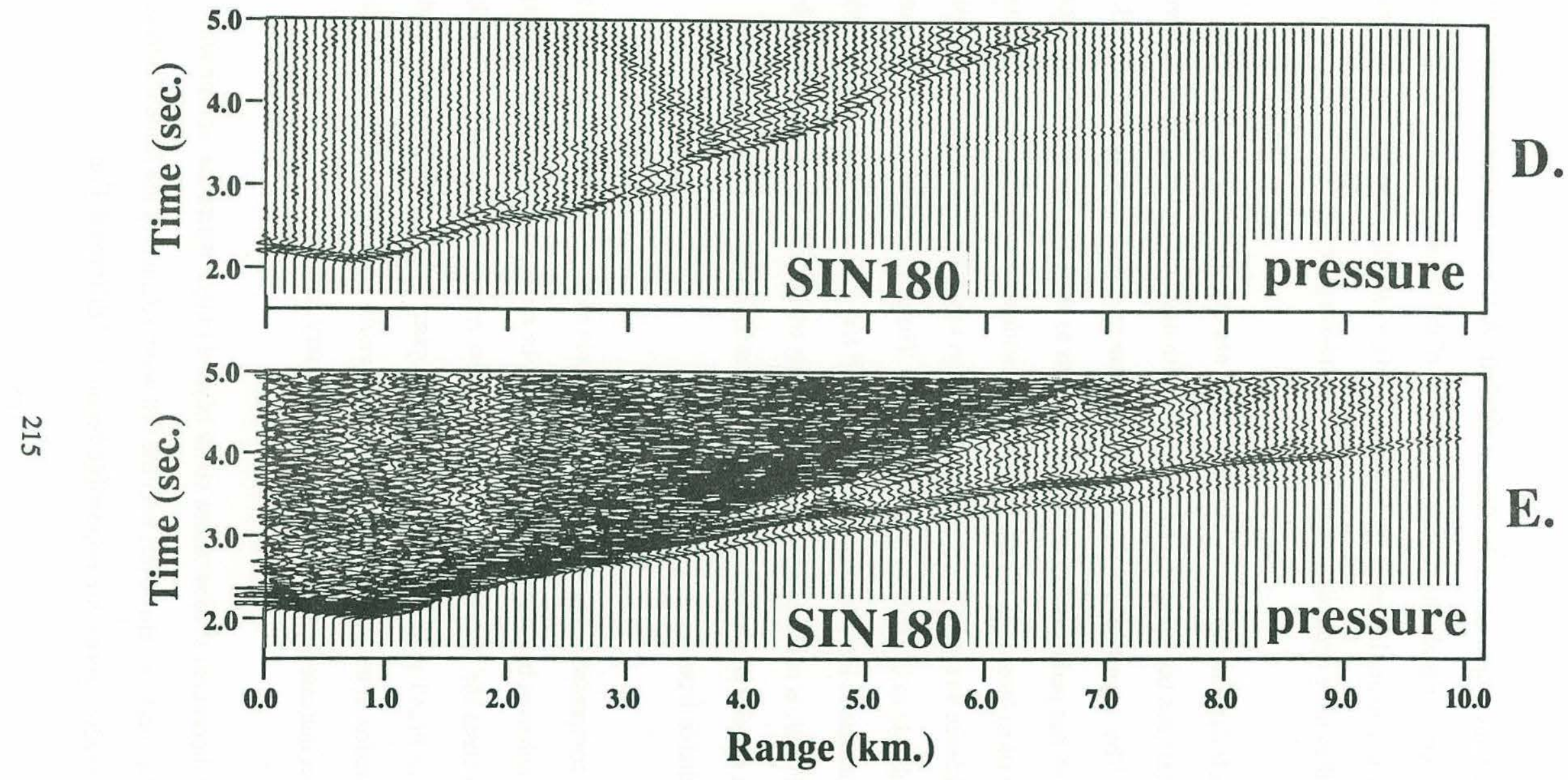


more dramatic travel time anomalies than in SIN360. Also note that the scattered noise in the seafloor receivers is about the same for all four of the initial models. This suggests that it is probably due to the 10 meters steps of the interface, which is about the same for all 4 models (i.e. they have approximately the same micro-roughness).

Models SIN90A and SIN90B were chosen not only to demonstrate shorter wavelength features but also to compare models with the same wavelength but different amplitudes. The snapshots and time series for these models are shown in figures 3.12 and 3.13. SIN90A has perhaps the most obvious effects as any of the initial models because of its relatively steep facets and wide fluctuations in incidence angle with range. Although the angle of incidence vs range curve for SIN90B has as many fluctuations, they are not as large in amplitude as in SIN90A (see figure 3.10). The principle effect of the fluctuations in angle of incidence is the presence of additional $\mathrm{P}$ and $\mathrm{S}$ diving waves. The P-diving arrival of SIN90A is made up of a number of diving waves with no distinct principle arrival. The shear diving wave arrival is also extremely incoherent and practically indistinguishable from 'noise'.

The compressional and shear diving waves in model SIN90B (figure 3.13) are both much more coherent but additional waves can still be resolved. The shallower topography of SIN90B causes the diving wave arrivals to appear more like those for the laterally homogeneous FLAT model of Dougherty and Stephen (1987,1988; chapters 1 and 2). Further reduction in the height of the topography would cause fewer additional diving waves and an increase in the coherence of the primary arrivals.

Ray theoretical shadow zones occur beyond ranges where the source wave is tangent to the seafloor. However, with the full wave solution of the finite difference technique, 'shadow zones' are impossible because of diffracted 'Franz-type' 
circumferential waves (Doolittle et al., 1967; Harbold and Steinberg, 1968; Neubauer, 1968). Areas in ray theory shadow zones occur at ranges greater than three kilometers in SIN90A but not until ranges greater than nine kilometers in SIN90B. This causes the direct and reflected arrivals of SIN90A to be less continuous than those of SIN90B. Backscattered reflected P-waves will occur whenever angle of incidence is equal to zero. These account for the first few strong backscattered arrivals in the water column receivers of the two models.

The seafloor receivers for both models contain bands of high amplitude interference patterns above valleys. This is most obvious for SIN90A (figure 3.12b-e). Since these bands do not show up in the water column receivers, they must be due to constructive interference of interface waves travelling in different directions over the topography. A less regular seafloor structure would not tend to produce these regular areas of interference because of less coherent interaction of the wavefronts.

A number of important scattering phenomena have been found in the previous models. It appears, however, that there are features of these models which cannot be due to scattering from the larger sinusoidal topography but must be due to scattering from smaller scale features such as the steps comprising the sloping seafloor. Intuitively, one can identify those scattering characteristics which are due to larger scale sinusoidal features (i.e. multiple diving waves, backscattered body waves for negative angle of incidence, and anomalies in travel time and amplitude) and those due to microroughness of the sloping seafloor (i.e. interface waves and the majority of scattered body waves). Also, if scattering from ten meter steps is indeed prominent, then it probably leads more to Bragg scattering problems than the regular nature of the sine wave (at least for the larger wavelength models). Fortunately, the superposition of ten meter features on larger scale topography is probably not unrealistic for many parts of the seafloor, particularly those 
Figure 3.12. Results from model SIN90A. 3.12a. The 4.0 second snapshot of compressional (top) and shear (bottom) energy. Note the presence of multiple P and S diving waves. $3.12 \mathrm{~b}, \mathrm{c}$. Waterborne receivers ( 500 meters above the seafloor baseline) for model SIN90A at two different amplitude scales. 3.12d,e. Pressure time series for seafloor receivers in model SIN90A at two different amplitude scales. 


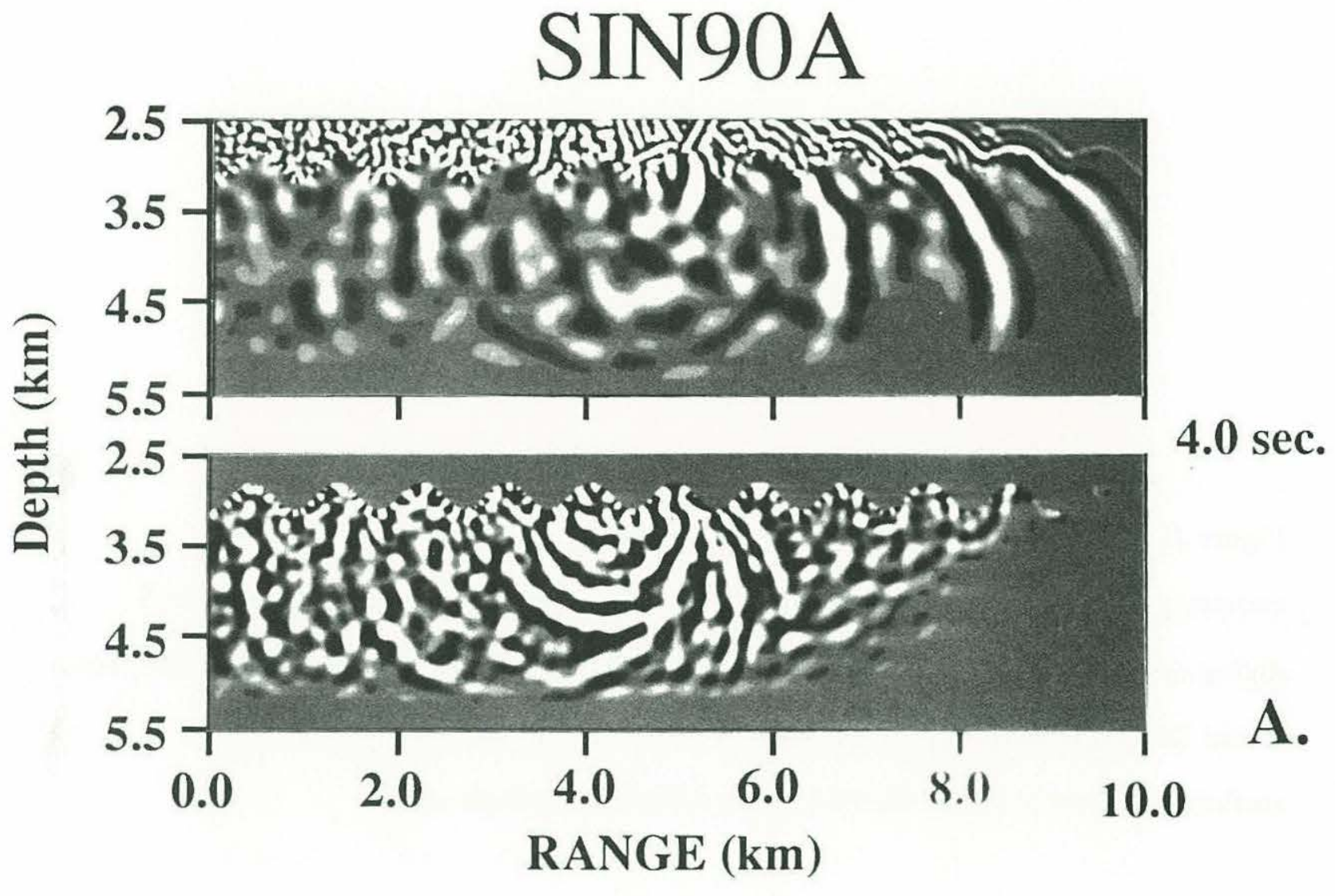


Figure 3.12. Results from model SIN90A. 3.12a. The 4.0 second snapshot of compressional (top) and shear (bottom) energy. Note the presence of multiple P and S diving waves. $3.12 \mathrm{~b}, \mathrm{c}$. Waterborne receivers ( 500 meters above the seafloor baseline) for model SIN90A at two different amplitude scales. 3.12d,e. Pressure time series for seafloor receivers in model SIN90A at two different amplitude scales. 

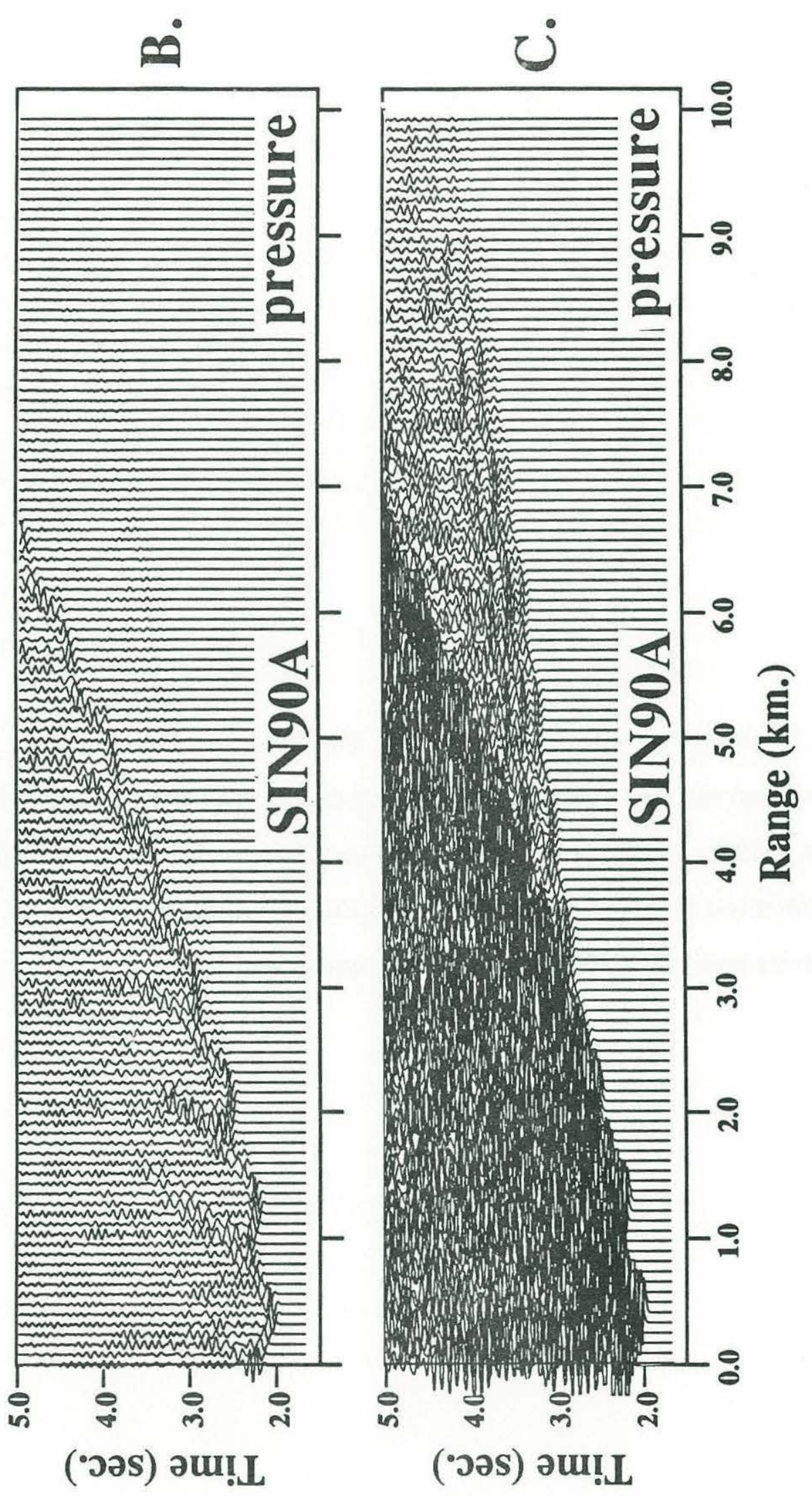
Figure 3.12. Results from model SIN90A. 3.12a. The 4.0 second snapshot of compressional (top) and shear (bottom) energy. Note the presence of multiple P and S diving waves. $3.12 \mathrm{~b}, \mathrm{c}$. Waterborne receivers ( 500 meters above the seafloor baseline) for model SIN90A at two different amplitude scales. 3.12d,e. Pressure time series for seafloor receivers in model SIN90A at two different amplitude scales. 

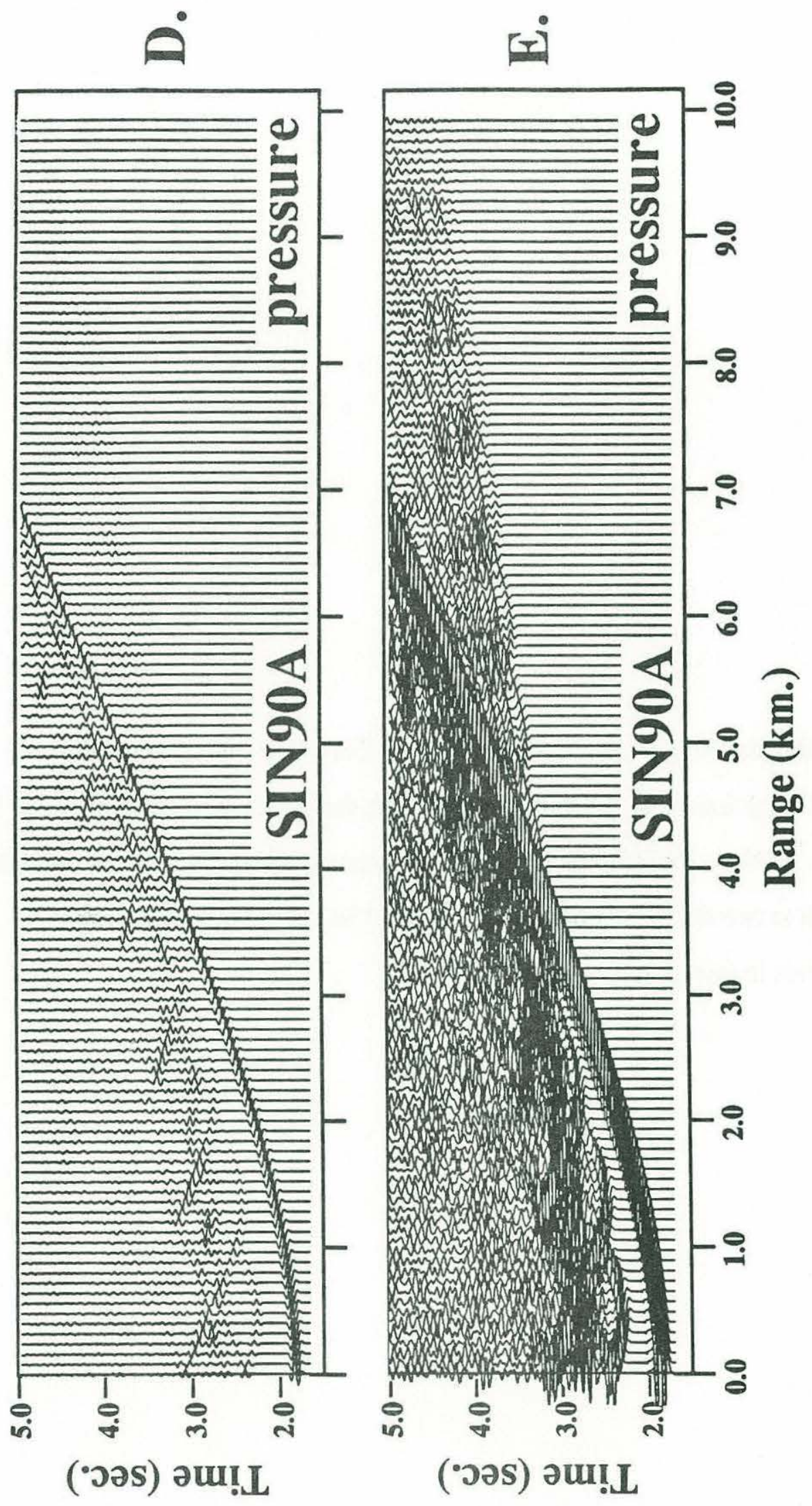
Figure 3.13. Results from model SIN90B. 3.13a. The 4.0 second snapshot of compressional (top) and shear (bottom) energy. Note the presence of multiple $\mathrm{P}$ and $\mathrm{S}$ diving waves. $3.13 \mathrm{~b}, \mathrm{c}$. Waterborne receivers ( 500 meters above the seafloor baseline) for model SIN90B at two different amplitude scales. 3.13d,e. Pressure time series for seafloor receivers in model S 


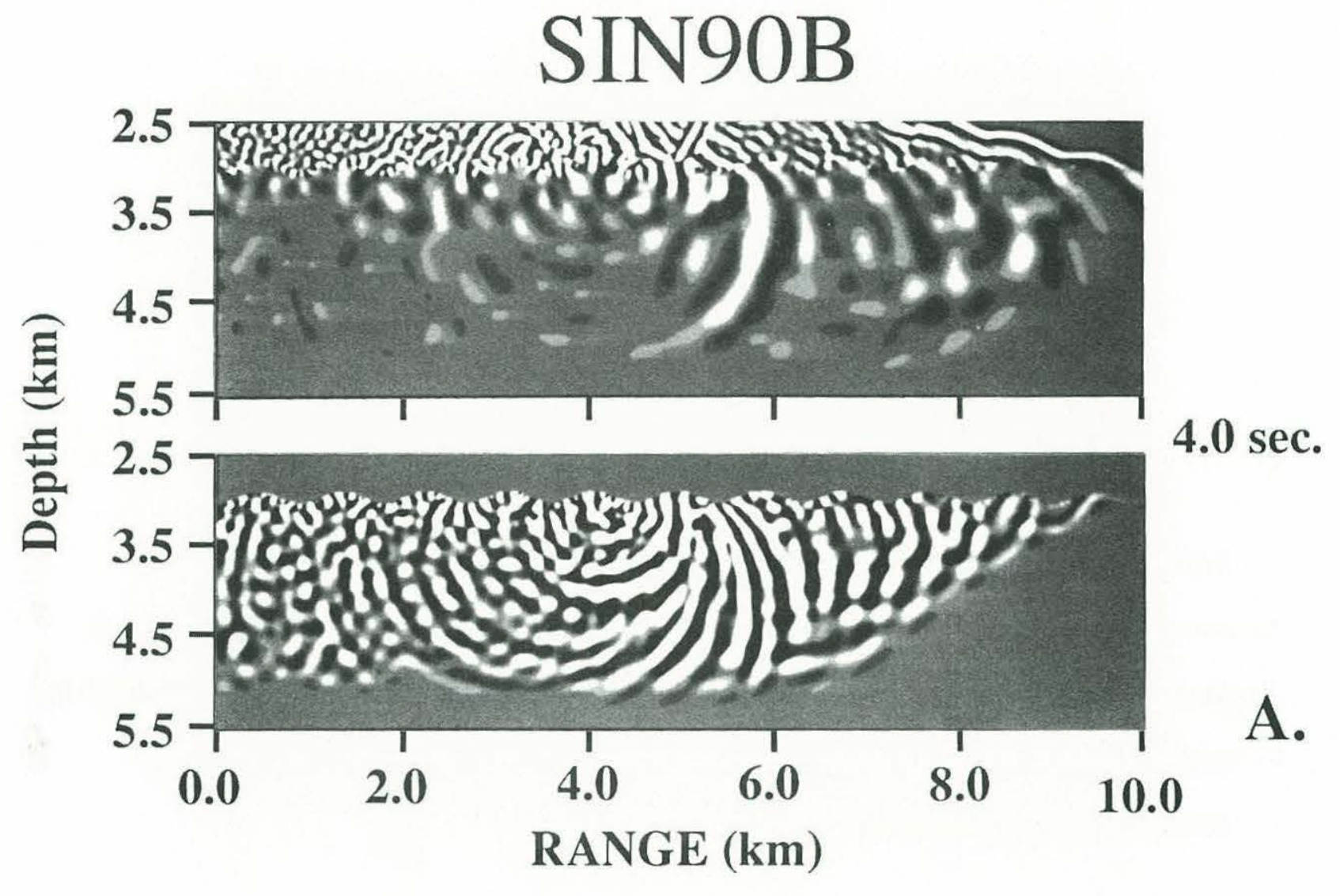


Figure 3.13. Results from model SIN90B. 3.13a. The 4.0 second snapshot of compressional (top) and shear (bottom) energy. Note the presence of multiple P and S diving waves. 3.13 b,c. Waterborne receivers ( 500 meters above the seafloor baseline) for model SIN90B at two different amplitude scales. 3.13d,e. Pressure time series for seafloor receivers in model S 

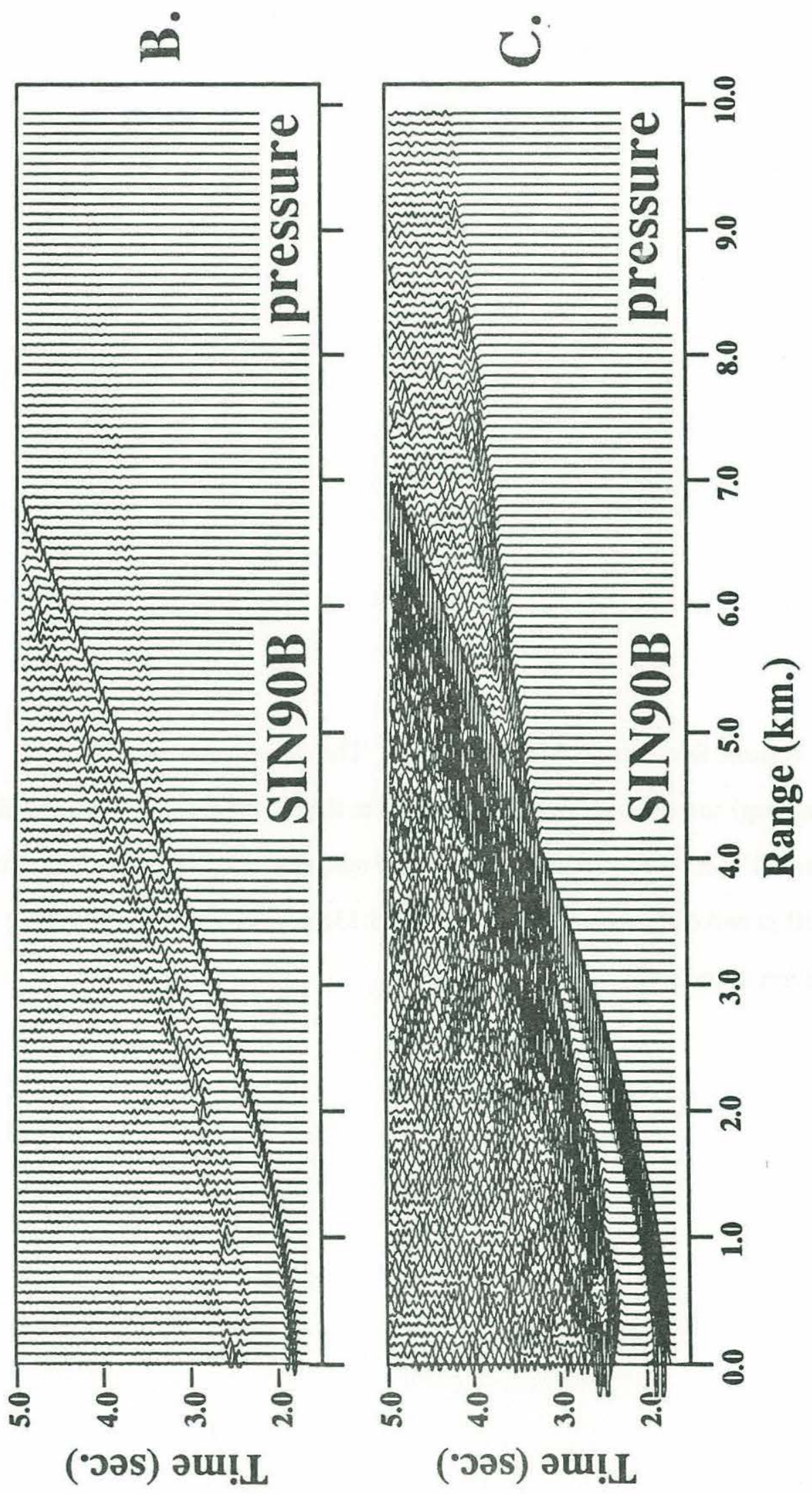
Figure 3.13. Results from model SIN90B. 3.13a. The 4.0 second snapshot of compressional (top) and shear (bottom) energy. Note the presence of multiple P and S diving waves. 3.13 b,c. Waterborne receivers ( 500 meters above the seafloor baseline) for model SIN90B at two different amplitude scales. 3.13d,e. Pressure time series for seafloor receivers in model S 

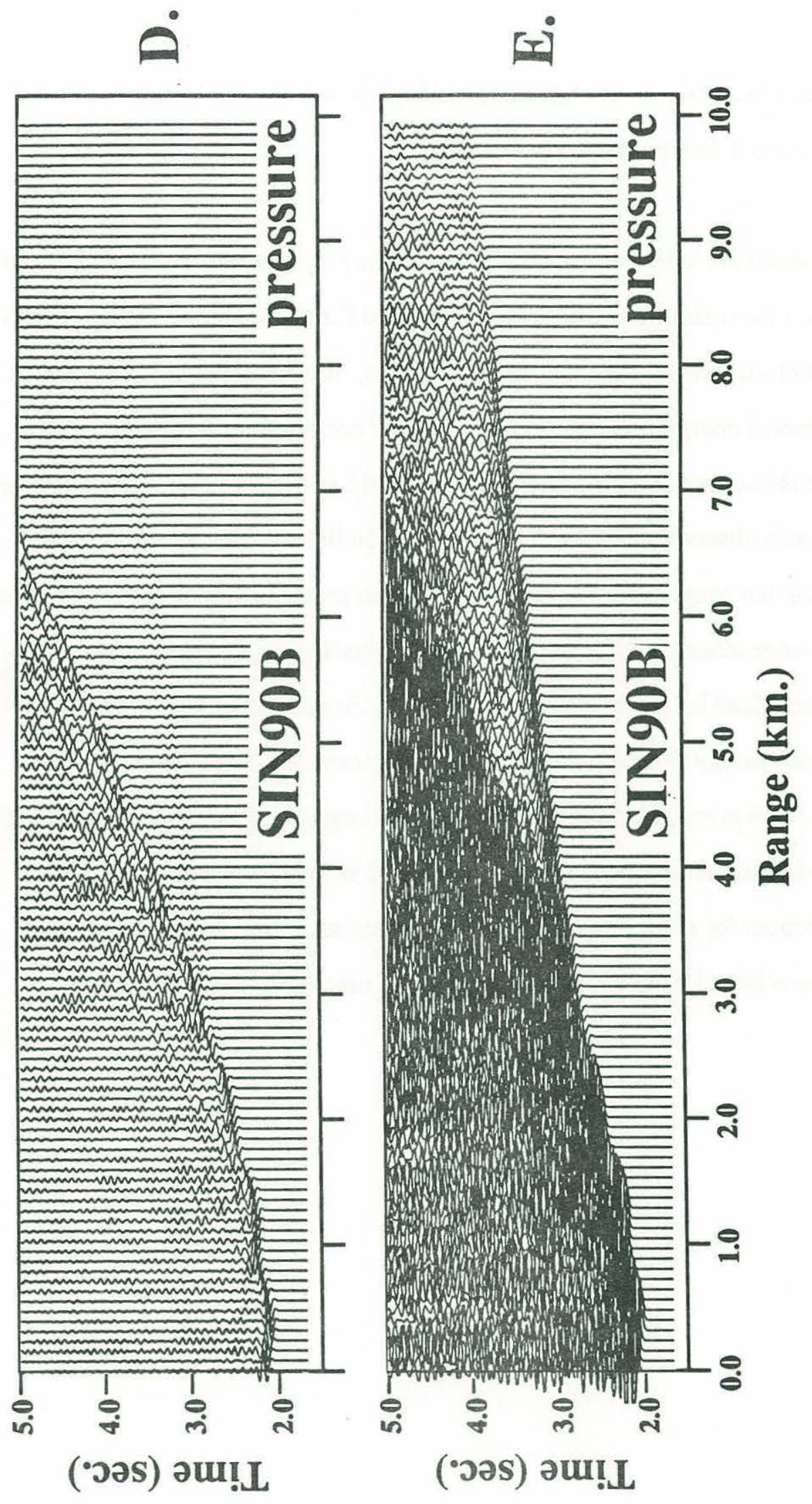
areas close to mid ocean ridges. Therefore, scattering phenomena due to this microroughness are not unexpected in these areas.

The characteristic 'criss-cross' pattern of scattering from small scale roughness on interfaces in this work is similar to that seen by Paul and Campillo (1988) in their work on the diffraction of elastic waves from corrugated surfaces. It is unclear from their results whether the diffracted energy is due entirely to the 'real' corrugations or if some of it is indeed due to the discretization of the interface. Hill and Levander (1984) and Levander and Hill (1985) also observed this pattern of scattering in finite difference models with small scale rectangular roughness. Levander (1988) also presents the results from a fourthorder finite difference scheme with a smoothly varying seafloor model (seafloor topography characterized by one cycle of a cosinusoid). In this model there is a large amount of scattered energy which is attributed solely to scattering from the large scale sinusoid feature. However, this feature is probably too large (topography amplitude $=100$ m., wavelength $=1.0 \mathrm{~km}$, and seismic frequency $=30 \mathrm{~Hz}$ ) with respect to the seismic wavelength to account for all of this energy. Some, if not most of this scattered energy must be due to the relatively large (10 meter) grid steps used to define the interface. 


\section{TEST MODELS}

The remainder of this chapter will be devoted to the determination of the validity and accuracy of scattering from the microroughness caused by the grid discretization of rough interfaces. Two tests were devised to investigate the accuracy of the finite difference solution to the rough seafloor problems. First, the grid increments were decreased in order to increase accuracy by allowing more grid points per wavelength and to more accurately describe a smoothly varying interface. The standard 15 points per wavelength used for the preliminary models is probably not fine enough to solve the sinusoidal model but may be sufficient to solve the actual stepwise interface imposed by the grid. The second way to check accuracy of the solution is by solving the reciprocal problem. A solution to the elastodynamic system should be reciprocal in both space and time (Aki and Richards, 1980 p.25-28). Two models with geometries which are reciprocal in space are presented.

\section{Finer grids}

The finite difference technique is a very computationally intensive method for the solution of the elastic wave equation. At the standard stability condition of ten points per wavelength, the computer power exists to solve realistic seismic refraction models relatively easily. Changing grid parameters can quickly increase the size of the model beyond the limits of an economic solution. Halving the grid step size (from ten to five meters) effectively increases the size of the model eight times. The number of grid steps in range and depth must both be doubled to result in a model with the same real range and depth in kilometers. In addition, the time step must also be halved (and the number of steps doubled) to preserve the stability condition in time (Dougherty and Stephen, 1988 (chapter 2); Kelly et al., 1976; Virieux, 1986). The computer resources available during 
this work allowed the grid dimension to be halved to five meters per step but did not allow for any grids at finer increments.

Two models were computed for the finer grid increment of five meters. Both of these models had the same large scale sinusoidal topography and velocity profiles as model SIN360. The first of these models, TEST1, used the same interpolation scheme to define the seafloor as in SIN360 but the smaller grid steps allowed a smoother interface to be defined (see figure 3.14). The small five meter steps should have a lesser effect on the 10 $\mathrm{Hz}$ seismic waves than did the ten meter steps of SIN360. The second model, TEST2, has the same ten meter step structure of the water-solid interface of SIN360 (see figure 3.14). However, the five meter spacing should provide a more accurate solution than the coarser ten meter grid.

TEST1 was slightly shorter in range than the initial models and has overall grid dimensions of 1900 by 550 grid points $(9.5 \times 2.75$ kilometers at five meters per grid point) with the same boundary and source conditions of the initial models (see figure 3.1). The distance between the bottom of the transition zone and the top of the telegraph region is slightly less for model TEST1 but this should not have a significant effect since we are interested mainly in interface effects. The model was run out to 8100 time steps (4.05 seconds at 0.0005 seconds/time step) to facilitate comparison of the 4.0 second snapshot of compressional and shear energy between the test models and SIN360. This snapshot for TEST1 is shown in figure 3.15a. In general, the amount of scattered energy is much less in TEST1 when compared to the 4.0 second snapshot of SIN360 (figure 3.6f). A few isolated scattered shear waves are still present in TEST1 but are of a much lesser energy level than most of the corresponding waves in SIN360. Also, the Stoneley waves at the interface are much lower in amplitude than in the previous models. Some scattering phenomena which are presumably due to the effect of the larger scale sinusoidal 


\section{Grid interfaces}

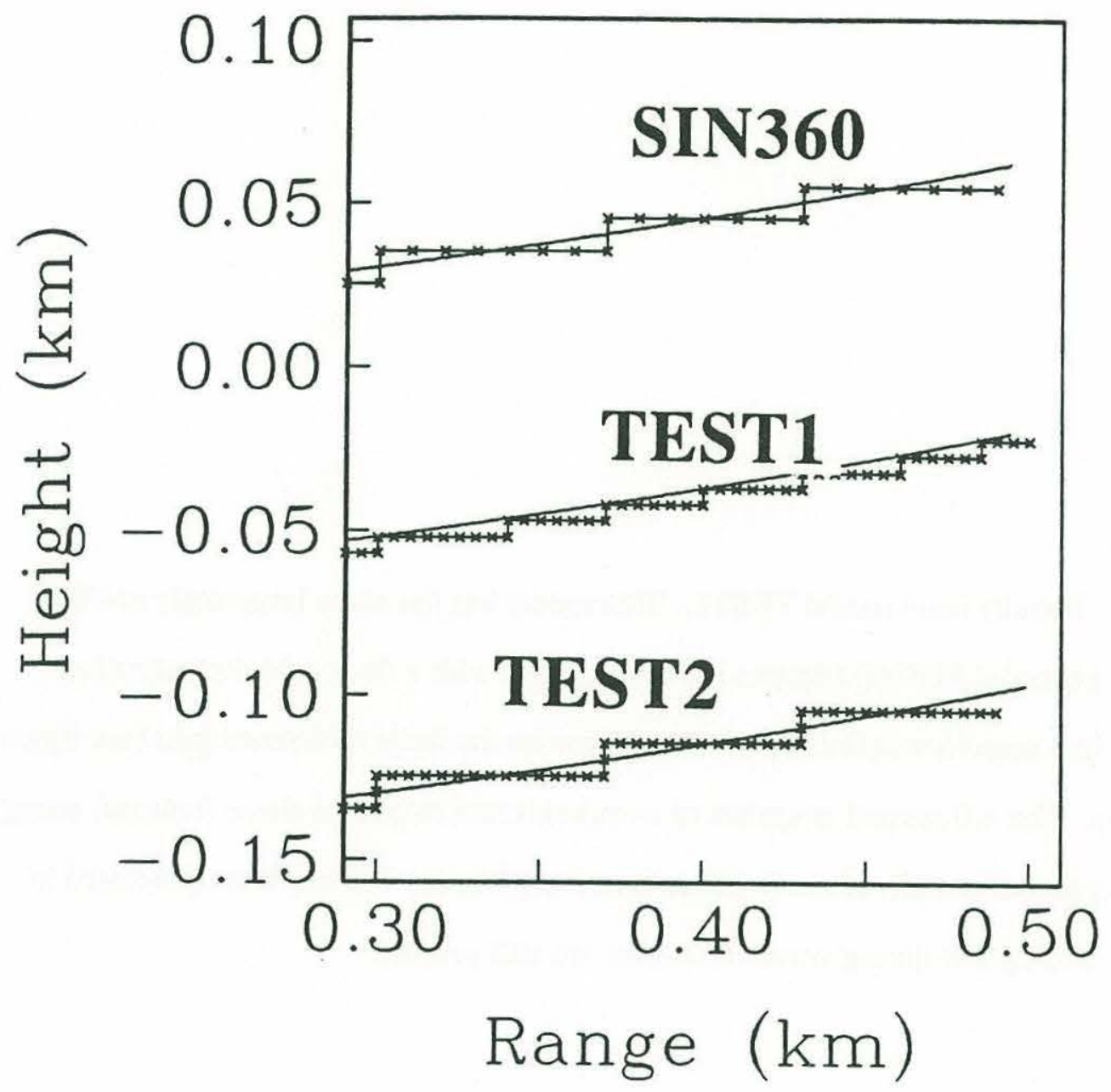

Figure 3.14. Close-up of the finite difference grid interfaces for models SIN360 (top), TEST1 (middle), and TEST2 (bottom). The grid interface is shown by the solid line with $x$ 's ( $x$ 's being located at grid points). The actual sinusoidal boundary is shown by the plain solid line. Grid spacing is 10 meters for SIN360 and 5 meters for models TEST1 and TEST2. 
Figure 3.15. Results from model TEST1. This model has the same large scale seafloor topography as model SIN 360 (figures 3.6 and 3.7) but with a finer grid discretization. This results in a smoother definition of the seafloor on the finite difference grid (see figure 3.14). 3.15a. The 4.0 second snapshot of compressional (top) and shear (bottom) energy. Note that much, but not all, of the incoherent scattered 'noise' has not been generated in this model. Multiple S-diving waves however, are still present. 


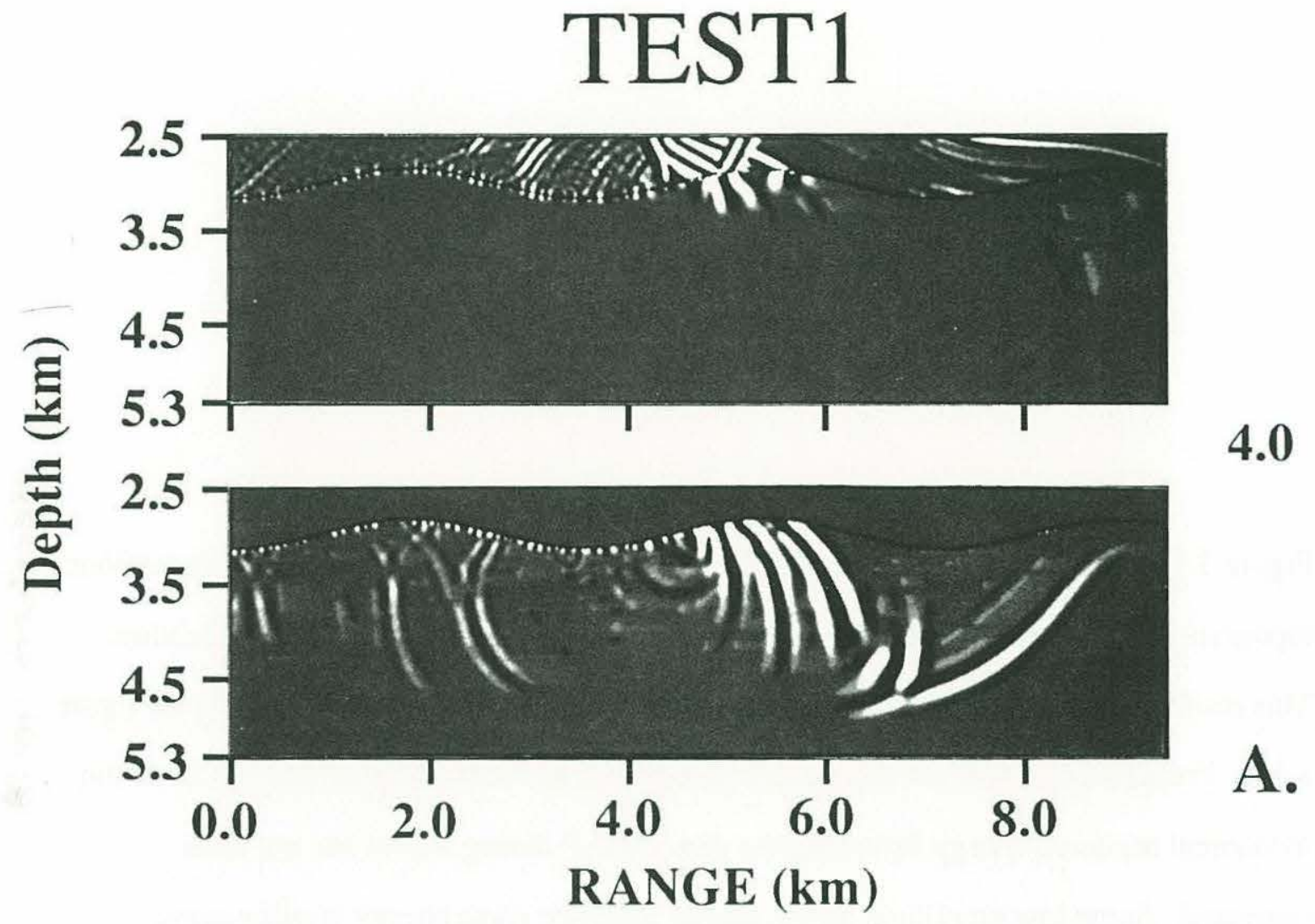


Figure 3.15b. Results from model TEST1. This model has the same large scale seafloor topography as model SIN360 (figures 3.6 and 3.7) but with a finer grid discretization. This results in a smoother definition of the seafloor on the finite difference grid (see figure 3.14). Presented here are the time series for seafloor receivers. Much, but not all of the incoherent scattered energy following the direct and P-diving waves has not been generated. Some low amplitude backscattered interface wave energy is still present following the direct wave. 


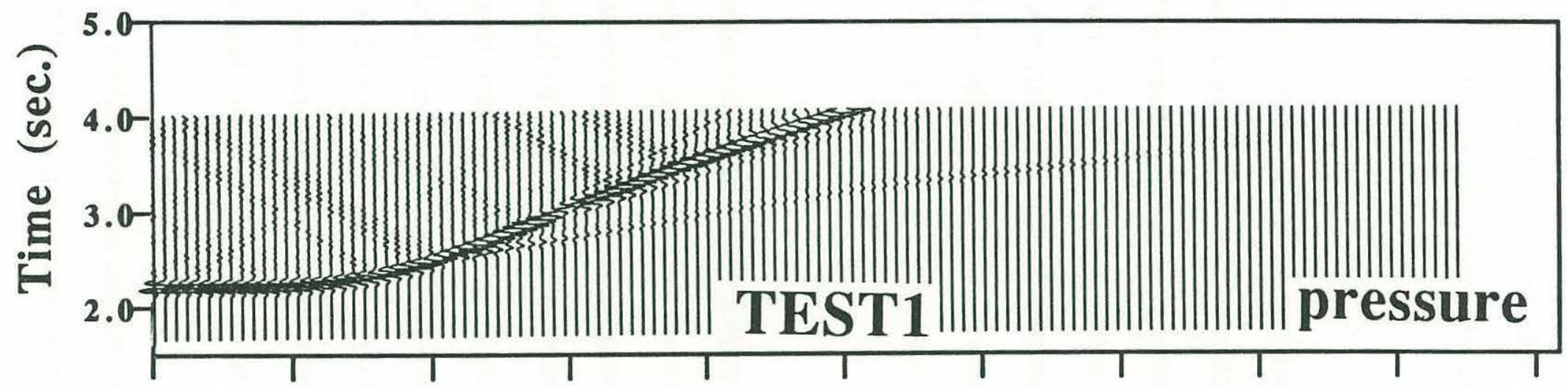

$\underset{\text { w }}{~}$

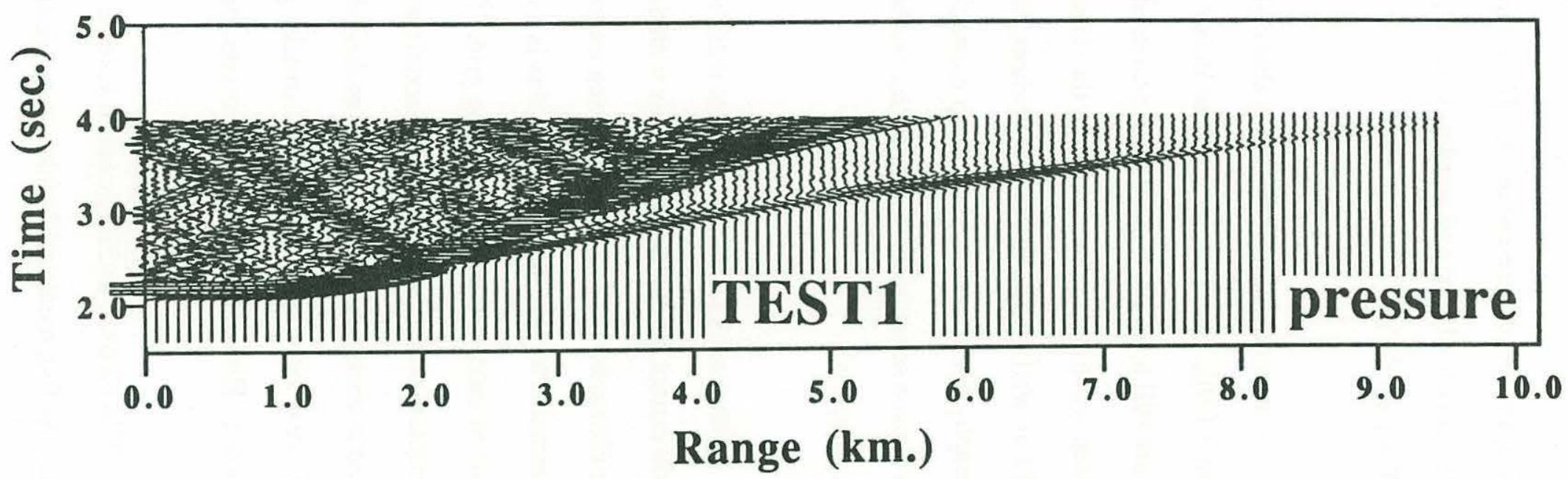


topography are still present in TEST1 as expected. These include additional shear diving waves, and travel time and amplitude anomalies of the principle arrivals (when compared to the FLAT model).

The same general results are also evident in the seafloor time series of TEST1 (figure 3.15b). The amplitude of the 'noise' following the direct wave is much reduced but there are still a few, low amplitude, scattered shear wave arrivals present in the time series. Interface wave amplitude following the direct wave is less with the finer step structure of TEST1 as well. Also, as in the snapshots, characteristics due to the larger scale topography are present and show up more clearly than in SIN360. Since only seafloor receiver time series were saved for this model, the signal from higher in the water column is not presented.

The results of TEST1 indicate that the majority of the backscattered energy in SIN360 cannot be due to interactions with the large scale topography. If this scattering is due to diffractions from the smaller ten meter steps on the finite difference seafloor, then what remains to be determined is if this is a realistic phenomenon at $10 \mathrm{~Hz}$ wavelengths, or is it due to inaccuracies of the coarse grid. The ideal procedure for obtaining an answer to this problem would be to calculate models with decreasing grid size until a constant solution is obtained. As mentioned earlier, the computer resources available limited this procedure to only one iteration with smaller grid increments (five meter spacing instead of 10 meter). However, useful conclusions can be drawn even from this limited test.

TEST2 used an interpolation scheme for seafloor definition which created ten meter steps on the five meter grid which were identical to the ten meter steps of SIN360 (see figure 3.14). Using this scheme, the diffractions from the coarse seafloor can be modelled more accurately on the finer grid. TEST2 has the same depth dimension as TEST1 (550 
points) but only reaches a range of 6.0 kilometers ( 1200 points at five meters per point). The model size is more than sufficient for close range (less than five kilometers) scattering.

The 4.0 second snapshot of compressional and shear energy and the seafloor pressure time series for model TEST2 are shown in figures $3.16 \mathrm{a}$ and $3.16 \mathrm{~b}$, respectively. The best qualitative comparison can be made with the 4.0 second snapshots of TEST2 (figure 3.16a) and SIN360 (figure 3.6f). In general, the strength of scattered energy is about the same in the two models. In both cases, scattering from the stepwise seafloor is much stronger that in the TEST1 model which has a smoother grid defined seafloor. A comparison between the seafloor pressure time series of models SIN360 (figure 3.7) and TEST2 (figure 3.16b.) also shows general agreement between scattered amplitudes. However, because TEST2 represents a more accurate solution to the problem (because of finer grid spacing), corresponding individual traces from the two models are not identical. Results from both the snapshots and time series indicate that features on the order of $1 / 15$ wavelength produce an important component of the scattered field.

\section{Reciprocal problem}

A second way to verify the accuracy of the finite difference formulation used is to compute the reciprocal problem. Because elastic wave propagation is a linear process, the signal generated between a source-receiver pair should be independent of direction. That is, the process of elastic wave propagtion is reciprocal in the spatial dimension (Aki and Richards, 1980, p.25-28). For example, within the same environment or model, the pressure signal received by a receiver at a range of 2.0 kilometers from a source at 0.0 kilometers should be the same as a signal received by a pressure receiver at 0.0 kilometers range from a source at 2.0 kilometers (for source and receivers at reciprocal heights and in 
Figure 3.16. Results from model TEST2. This model has the same large scale sinusoidal seafloor structure as models SIN360 and TEST1. However, the finer discretization of the seafloor in model TEST2 follows the coarse steps of model SIN360 (see figure 3.14).

3.16a. The 4.0 second snapshot of compressional (top) and shear (bottom) energy. Incoherent scattered energy (scattering from stepwise seafloor discretization) is generated and is as strong as in model SIN360 (see figure 3.6). 


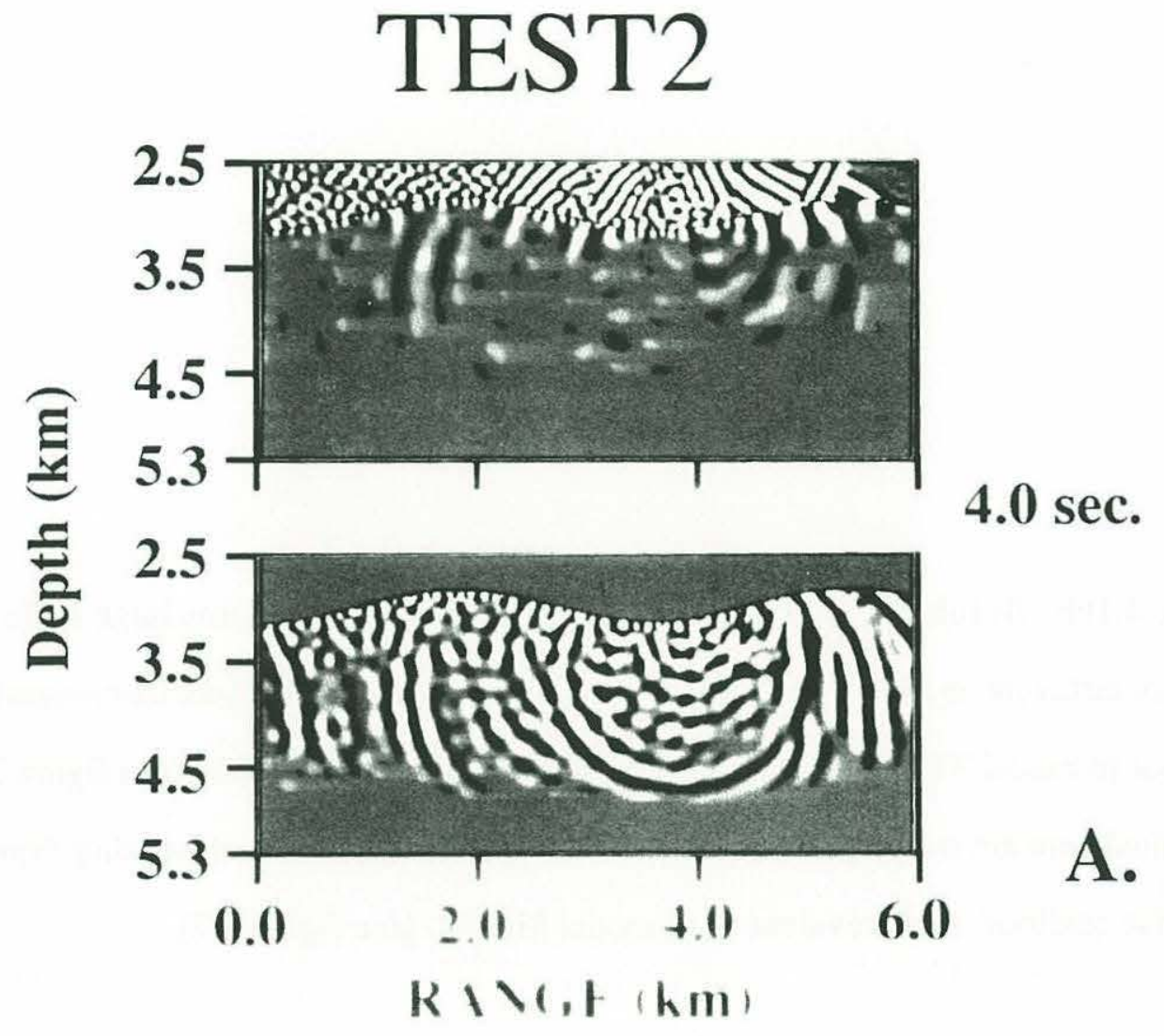


Figure 3.16b. Results from model TEST2. This model has the same large scale sinusoidal seafloor structure as models SIN360 and TEST1. However, the finer discretization of the seafloor in model TEST2 follows the coarse steps of model SIN360 (see figure 3.14). Presented here are the pressure time series for seafloor receivers. Scattering from the stepwise seafloor is as prevalent as in model SIN360 (see figure 3.7). 

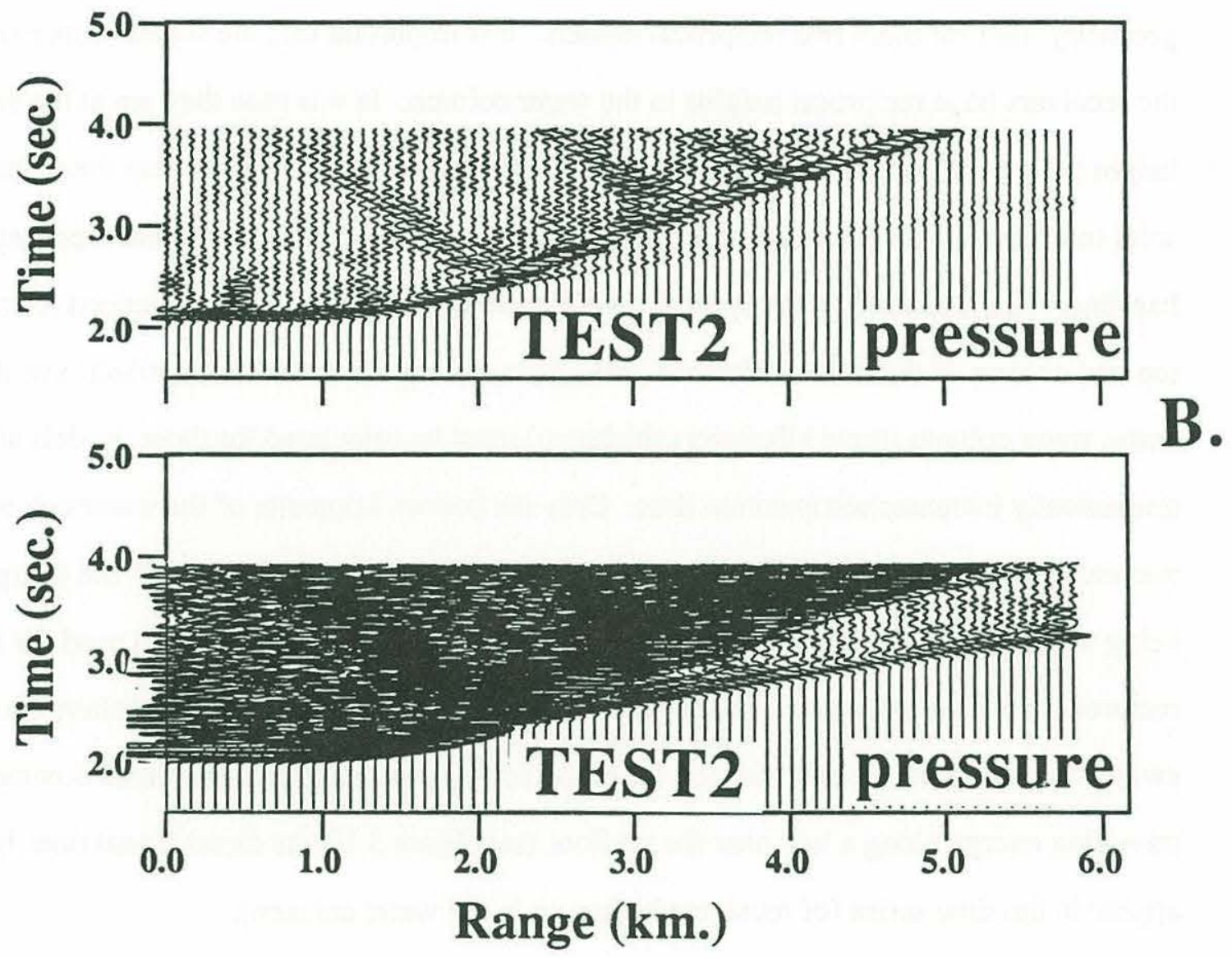
a fluid).

In order to calculate this problem using our formulation, a few changes had to be made on the grid used for all of the previous models. Figure 3.17 shows the grid geometry used for these two reciprocal models. It is important that the actual source and the receivers be at reciprocal heights in the water column. In this case they are at the same height because this formulation does not allow for placing the source too near the watersolid interface. The source and receivers are located 1.5 kilometers above the topography baseline. This was used as the optimal level location to minimize false reflections from the top and corners of the finite difference grid. Also, to help minimize false reflections, the entire water column (three kilometers thickness) must be calculated for these models which dramatically increases computation time. Only the bottom kilometer of the water column was calculated using finite differences in the previous models with the rest of the energy being absorbed by the top boundary. This top absorbing boundary was also used for the reciprocal problem but since it is not perfectly absorbing, it was necessary to place it as far away from the receivers as possible. Since the source is actually introduced as downward travelling energy along a line near the seafloor (see figure 3.13) its direct signal does not appear in the time series (of receivers higher up in the water column).

Two models were run (RECIP1 and RECIP2) which had reciprocal structure and geometry. A grid dimension of 10 meters was used for both models with a time step of 0.001 second. A constant velocity crust $(3.2 \mathrm{~km} / \mathrm{sec}$ for P-waves, $1.85 \mathrm{~km} / \mathrm{sec}$ for Swaves) beneath the sinusoidal topography was used rather than the gradients and lateral heterogeneity profiles of the previous models. This was done to isolate effects of the small interface steps from any energy turning in the gradients. Both models used the same interface structure as SIN360 (topography amplitude $=150$ meters, wavelength $=3.6$ kilometers) with receivers located every 40 meters. Although only a few receivers will be 
used for the reciprocity results, the entire line was saved and used for identification of arrivals. The topography of RECIP2 used the reciprocal sinusoid to RECIP1 (i.e. a phase shift of 180 degrees was added, see figure 3.17). The periodic nature of the seafloor in both models allows more than one source-receiver pair in each model to overlap with the corresponding pair in the other model. RECIP1, with its source over a valley and receivers at 1.8 and 5.4 kilometers (and so on every $3.6 \mathrm{~km}$. in range) over hills is reciprocal to RECIP2, with its source over a hill and receivers at 1.8 and 5.4 kilometers (and out) over valleys. The signals from the 1.8 kilometer receivers of the two models should be the same, as well as those for the 5.4 kilometer receivers.

It is important to understand that the time series from all of the corresponding receivers in the two models will not be similar, only those source-receiver pairs which have reciprocal geometry. Figure 3.18 presents the time series signals from two reciprocal receiver pairs at 1.8 and 5.4 kilometers in models RECIP1 and RECIP2. In both cases, the agreement is very good, especially at earlier times in the time series. Slight discrepancies at later times are due to two factors. First, it has already been shown that the 10 meter solution is slightly inaccurate when compared to the 5 meter solution to the rough interface problem. Therefore, some directional inaccuracies may be present in the reciprocal models. A larger source of disagreement in the later sections of the time series is due to boundary problems. Although the absorbing boundaries are considered to be quite efficient in absorbing energy, they are not perfect and add to the signal at late times. The receivers were placed as far away as possible from any problem areas but still received some boundary noise. Since the geometry which includes the seafloor, source-receiver pair, and grid boundaries is not reciprocal, any corner or edge reflections will not correspond in the two models. 
Figure 3.17. Grid layout for the reciprocal problem. The entire water column is calculated using finite differences for this problem. Crust below the seafloor is of a constant velocity to isolate seafloor scattering effects. Note that while the source-seafloor-receiver geometries in the two models are reciprocal, the relationship between the source-receivers and the artificial numerical boundaries and corners is not. Reciprocal traces occur at 1.8 and $5.4 \mathrm{~km}$. range ( and so on every $3.6 \mathrm{~km}$.). 


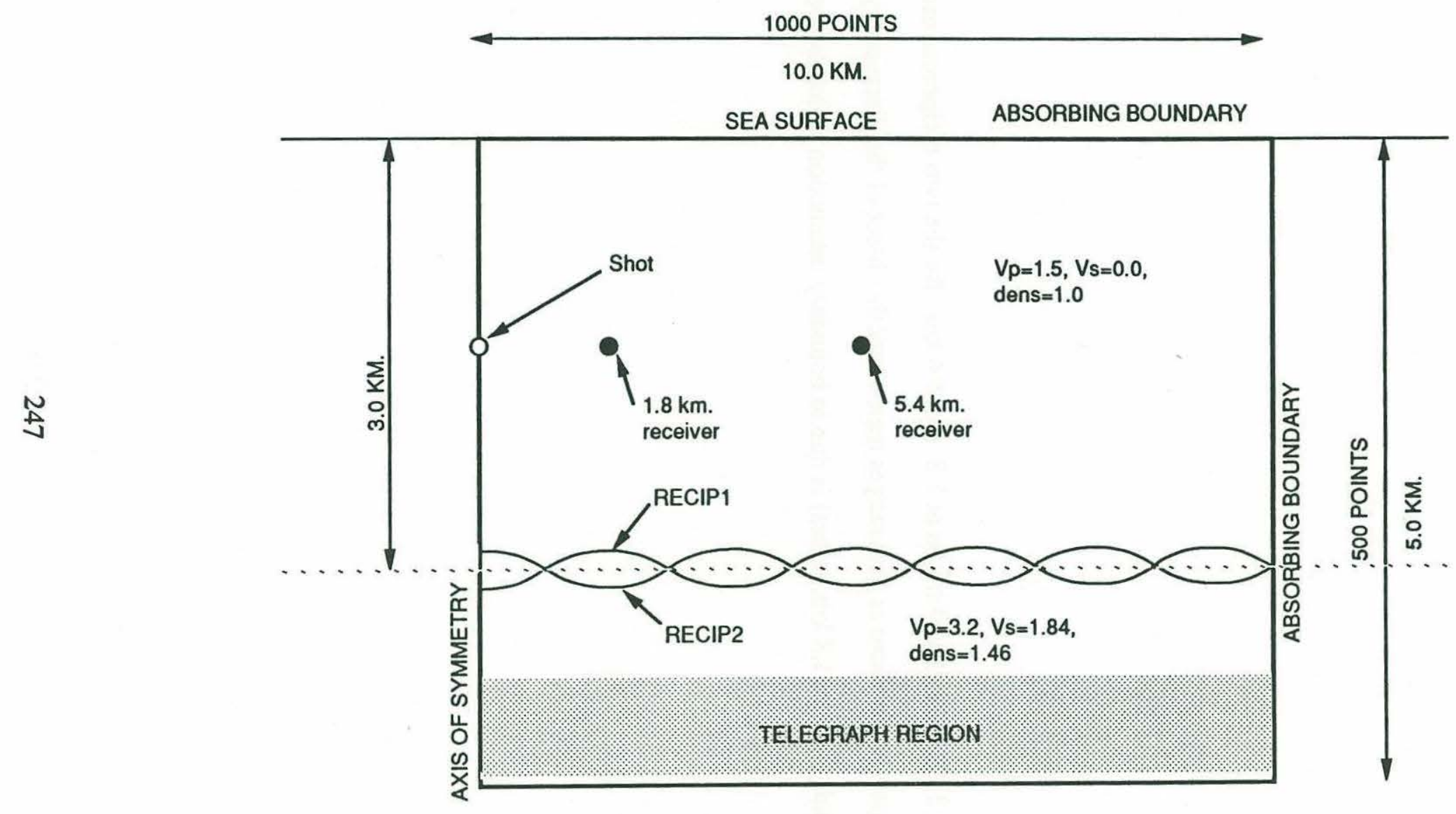


Figure 3.18. Reciprocal traces at 1.8 and $5.4 \mathrm{~km}$. for the two reciprocal models. The initial part of the traces at both ranges match exactly. Most of the discrepancy at later times (especially for the $1.8 \mathrm{~km}$. traces) is due to boundary interaction which is not reciprocal. 


\section{RECIPROCAL MODELS}

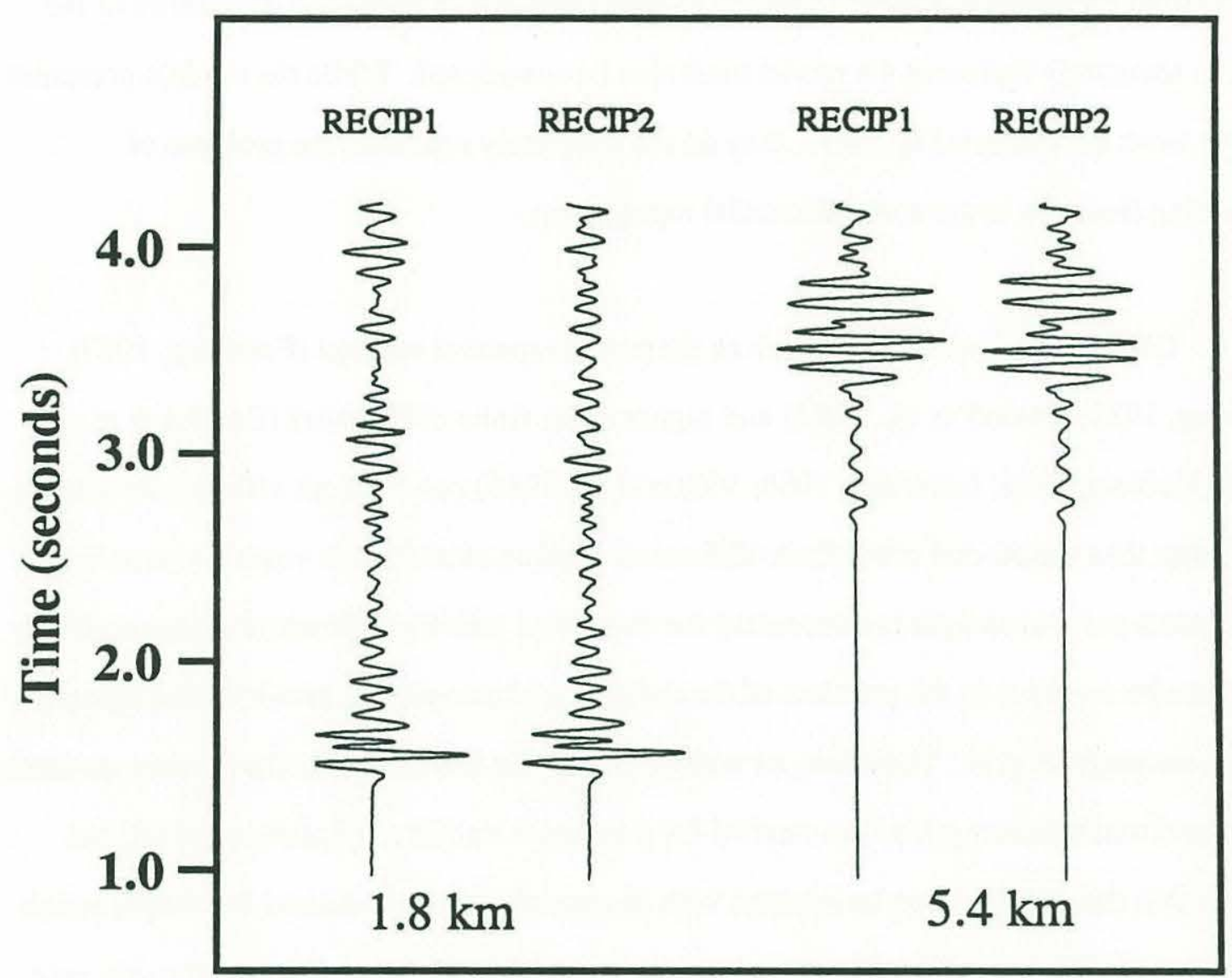


The results from the test models point out an important numerical result of this work. That is, the need to consider more than numerical stability and dispersion when determining the necessary grid spacing. Since features with sizes on the order of $1 / 15$ of the seismic wavelength have been shown to cause significant scattering, the ability of the grid to accurately represent the model must also be considered. While the models presented in this work are numerically stable, they do not accurately represent the problem of scattering from the larger scale sinusoidal topography.

Other numerical methods such as the pseudo-spectral method (Fornberg, 1987; Gazdag, 1981; Kosloff et al., 1984) and higher order finite differences (Bayliss et al., 1986; Dablain, 1986; Levander, 1988; Vidale et al., 1985) can be more efficient for certain problems than the second order finite difference scheme used for this work because fewer grid points per wavelength are necessary for numerical stability. However, these methods will also be sensitive to the problem of the definition of non-planar, non-level topography with a rectangular grid. Therefore, as with second order formulations, these other methods will need much denser grids than needed for numerical stability. (Fornberg, 1988) has shown that this problem can be avoided with the pseudo-spectral method for simpie rough interfaces by deforming the grid around the interface so that the interface lies directly on a grid line (no 'stair-stepping' needed for non-level interfaces). Some of the efficiency of that method is lost by using deformed grids but the method shows good results for simple rough interfaces between homogeneous media. However, logical deformation of grids for more complicated 2-D structures and velocity profiles is probably not realistic. 


\section{CONCLUSIONS}

The finite difference models of scattering from sinusoidal seafloor topography presented in this paper have demonstrated the effects of scattering from both macro- and micro-roughness, as well as some important constraints on the method used. The initial models, run with relatively coarse grids show that effects such as travel time anomalies, additional compressional and shear diving waves and some strong back reflected arrivals are due to the rough topography. Steep topography allows energy, especially shear energy, to enter the seafloor even at great ranges. Ray theoretical shadow zones are not present because of Franz-type waves diffracted into areas where the grazing angle is less than zero.

It was suspected from the initial model results that not all of the scattered signal could be due to the large scale sinusoidal topography. Phenomena such as backscattered body waves (predominantly shear waves), scattering into interface waves, and strong interference effects are most likely due to the grid imposed micro-roughness superimposed onto the larger scale topography. Seen in a vertical seismic profile geometry, the backscattered, near horizontally travelling S-waves appear very similar to arrivals which have previously been interpreted as near vertically travelling P-waves reflected from layers deep in the crust. All of these scattered wave types are important because of the abundance of smaller scale features on the sea floor especially near mid-ocean ridges.

Models with finer grids and both smooth and coarse water-solid interfaces demonstrated that scattering from interface steps on the order of $1 / 15$ wavelength is realistic and important. However, even finer grids (>30 points/wavelength) are necessary for better definition of a smooth seafloor (without significant micro-roughness). The 
reciprocal problem computed with a coarse grid (15 points/wavelength) demonstrated that the coarse grid provides a realistic simulation of wave propagation through sinusoidal topography with grid imposed small scale topography. 


\section{REFERENCES}

Achenbach, J. D., Gautesen, A.K., and McMakem, H., (1982), Ray methods for waves in elastic solids, Pitman Publ. Inc.

Akal, T., (1978), Two-dimensional space series analysis for sea-floor roughness, Mar. Geotech., 3(2), 171-182.

Aki, K., and Richards, P. G., (1980), Quantitative Seismology, W.H. Freeman and Co., San Francisco, 932p.

Bayliss, A., Jordan, K.E., LeMesurier, B.J., and Turkel, E., (1986), A fourth-order accurate finite-difference scheme for the computation of elastic waves, Bull. Seis. Soc. Am., 76, 1115-1132.

Becker, K., Sakai, H., et al., (1988), Proc, ODP. Init. Repts. (Pt. A), 111: College Station, TX (Ocean Drilling Program).

Bell, T. H., (1975), Statistical features of the sea - floor topography, Deep - Sea Res., 22, 883-892.

Bell, T. H., (1979), Mesoscale sea floor roughness, Deep - Sea Res., 26A, 65-76.

Bouchon, M., (1985), A simple, complete numerical solution to the problem of diffraction of SH waves by an irregular boundary, J. Acoust. Soc. Am., 77, 1-5.

Campillo, M. and Bouchon, M., (1985), Synthetic SH seismograms in a laterally varying medium by the discrete wavenumber method, Geophys. J. Roy. astr. Soc., 83,

Clayton, R. and Engquist, B., (1977), Absorbing boundary conditions for acoustic and elastic wave equations, Bull. Seis. Soc. Am., 67, 1529-1540.

Dablain, M. A., (1986), The application of higher-order differencing to the scalar wave equation, Geophysics, 51, 54-66.

Doolittle, R.D., Überall, H., and Ugincius, P, (1967), Sound scattering by elastic 
cylinders, J. Acoust. Soc. Am., 43, 1-14.

Dougherty, M. E. and Stephen, R. A., (1987), Geoacoustic scattering from seafloor features in the ROSE area, J. Acoust. Soc. Am., 82, 238-256.

Dougherty, M. E. and Stephen, R. A., (1988), Seismic energy partitioning and scattering in laterally heterogeneous ocean crust, PAGEOPH, 128(1/2), 195-229.

Ewing, J. I. and Purdy, G. M., (1982), Upper crustal velocity structure in the ROSE area of the East Pacific Rise, J. Geophys. Res., 87, 8397-8402.

Fornberg, B., (1987), The pseudospectral method: Comparisons with finite differences for the elastic wave equation, Geophysics, $52,483-501$.

Fornberg, B., (1988), The pseudospectral method: Accurate representation of interfaces in elastic wave calculations, Geophysics, 53(5), 625-637.

Gazdag, J., (1981), Modeling of the acoustic wave equation with transform methods, Geophysics, 46, 854-859.

Harbold, M. L. and Steinberg, B. N., (1968), Direct experimental verification of creeping waves, J. Acoust. Soc. Am., 45(3), 592-603.

Hill, N. R. and Levander, A. R., (1984), Resonances of low-velocity layers with lateral variations, Bull. Seis. Soc. Am., 74, 521-537.

Kelly, K. R., Ward, R.W., Treitel, Sven, and Alford, R.M., (1976), Synthetic seismograms: A finite difference approach, Geophysics, 41, 2-27.

Kennett, P., Ireson, R.L., and Conn, P.J., (1980), Vertical seismic profiles: Their application in exploration geophysics, Geophys. Prospecting, 28, 676-699.

Kosloff, D., Reshef, M., and Loewenthal, D., (1984), Elastic wave calculations by the Fourier method, Bull. Seis. Soc. Am., 74, 875-891.

Levander, A. R., (1988), Fourth-order finite difference P-SV seismograms, Geophys., 53(11), 1425-1436.

Levander, A. R. and Hill, N. R., (1985), P-SV resonances in irregular low velocity surface layers, Bull. Seis. Soc. Am., 75, 847--864. 
Luppé, F. and Doucet, J., (1988), Experimental study of the Stoneley wave at a plane liquid-solid interface, J. Acoust. Soc. Am., 83(4), 1276-1279.

Menard, H.W., (1964), Marine geology of the Pacific. McGraw-Hill, 271 p.

Neubauer, W. G., (1968), Pulsed circumferential waves on aluminium cylinders in water, J. Acoust. Soc. Am., 45(5), 1134-1144.

Neubauer, W. G., (1986), Acoustic Reflection from surfaces and shapes, Washington, D.C., Naval Research Laboratory.

Ogilvy, J. A., (1987), Wave scattering from rough surfaces, Rep. Prog. Phys., 50, 15531608.

Paul, A. and Campillo, M., (1988), Diffraction and conversion of elastic waves at a corrugated interface, Geophysics, 53(11), 1415-1424.

Purdy, G. M., (1982a), The variability in seismic structure of layer 2 near the EPR at 12 N, J. Geophys. Res, 87, 8403-8416.

Purdy, G. M., (1982b), The correction for the travel time effects of seafloor topography in the interpretation of marine seismic data, J. Geophys. Res., 87, 8389-8396.

Rayleigh, L., (1878), The Theory of Sound, London, Macmillan.

Scholte, J. G., (1947), The range of existence of Rayleigh and Stoneley waves, Roy. astr. Soc. Mon. Not. Geophys. Supp., 5, 120-126.

Stephen, R. A., (1983), The oblique seismic experiment of DSDP leg 70, Initial Rep. Deep Sea Drill. Proj., 69, 301-308.

Stephen, R. A., (1984), Finite difference seismograms for laterally varying marine models, Geophys. J. R. astr. Soc., 79, 184-198.

Stephen, R. A., (1984), Synthetic veritcal seismic profiles by the method of finite differences. In Toksoz, M. N. and Stewart, R.R., Vertical Seismic Profiling, Part B: Advanced Concepts, Geophysical Press, Amsterdam, p. 63-79.

Stephen, R. A.,(1988a), Finite difference methods for bottom interaction problems, Computational Acoustics: Wave Propagation,D. Lee, R. L. Sternberg 
and M. H. Schultz,225-238.

Stephen, R.A., (1988b), Lateral heterogeneity in the upper oceanic crust at DSDP Site 504, J. Geophys. Res., v. 93, p.6571-6584.

Stephen, R.A., (1988c), A review of finite difference methods for seismo-acoustic problems at the sea floor, Reviews of Geophysics, v.26, p.445-458.

Stephen, R. A., Louden, K.E., and Matthews, D.H., (1979), The oblique seismic experiment on Deep Sea Drilling Project leg 52, Initial Rep. Deep Sea Drill. Proj., v.51-53, 675-704.

Stephen, R. A., Louden, K.E., and Matthews, D.H., (1980), The oblique seismic experiment on DSDP leg 52, Geophys. J. R. astron. Soc., 60, 289-300.

Stoneley, R., (1924), Elastic waves at the surface of separation of two solids, Proc. R. Soc. Lond., 106(A), 416-428.

Vidale, J., Helmberger, D.V., and Clayton, R.W., (1985), Finite-difference seismograms for SH waves, Bull. Seis. Soc. Am., 75, 1765-1782.

Virieux, J., (1984), SH-wave propagation in heterogeneous media: Velocity-stress finitedifference method, Geophysics, 49, 1933-1957.

Virieux, J., (1986), P-SV wave propagation in heterogeneous media: Velocity-stress finite difference method, Geophysics, 51(4), 889-901. 


\section{CHAPTER 4}

\section{A time domain solution to acoustic wave scattering from an infinite elastic cylinder}




\section{A time domain solution to acoustic wave scattering from an infinite elastic cylinder.}

\section{INTRODUCTION}

This chapter presents a time domain solution to the problem of acoustic wave scattering from an infinite elastic cylinder by using the finite difference method. It is difficult to imagine how such a conceptually 'simple' problem has been the subject of so much interest and elucidation over the years. This is a very well studied problem and has been so for over 100 years, beginning with the efforts of the eminent nineteenth century acoustician, Lord Rayleigh (Rayleigh, 1878). Our purpose for trying this problem is not to shed any new light onto the mechanisms of scattering or to discover any heretofore unknown scattered modes, but rather, to use the problem as a stringent test of the finite difference method used for other, more geologically relevant scattering problems (Dougherty and Stephen, 1988 (chapter 2); Stephen, 1988; Virieux, 1984; Virieux, 1986).

In a time domain sense, two phenomena characterize the problem of scattering from a cylinder. First, the backscattered signal received after the illumination of an infinite cylinder by an incident pulse source contains not a single reflected arrival but a number of pulses arriving after the initial specular reflection. Also, insonification by a CW (continuous wavelet) source results in a complex interference pattern of energy scattered in a number of discrete directions from the cylinder. Lord Rayleigh first studied this problem for the cases of rigid and acoustic cylinders in a fluid medium and found that much of the reverberated energy was due to the existence of diffracted waves which travel along the 
circumference of the cylinder. These results were expanded to the elastic cylinder case by Faran (1951) and Lowan (1946) who found that even more circumferential wave modes are possible when the cylinder also supports shear. The situation for nonrigid and elastic cylinders is complicated further by the presence of transmitted and whispering gallery modes as seen by Doolittle et al. $(1966,1968)$ and Brill and Überall $(1970)$.

Solutions to the cylindrical scattering problem have been obtained by a number of analytical, experimental, and numerical methods. Analytically, the problem has been solved by two basic techniques for cylinders with radii near the size of the acoustic wavelength or larger. Rayleigh (1878), Lowan et al. (1946), and Faran (1951) presented solutions which were comprised of series of normal modes of the cylinder. This method produces a solution which implicitly contains all possible wavetypes scattered by the cylinder if enough modes are included. The disadvantage of this method is that individual wave types which contribute to the total scattered field cannot easily be individually examined. Also, a fair amount of computation is involved in making the series converge. Other authors, (Doollittle et al., 1966,1967, Brill and Überall, 1970, and Neubauer, 1986) have employed the Sommerfeld-Watson transformation (Watson, 1919) which transforms the infinite normal mode series expression for the scattered pressure into a contour integral in the complex mode number plane. While this solution may converge more quickly than the normal mode solution, the zeros must still be found numerically. The disadvantages of this method are that it is not a total solution (unless all of the zeros are found) and that the numerical search for the poles may be complicated, especially for small cylinder radii (Frisk et al., 1975; Grace and Goodman, 1966). Numerical experiments have verified theoretical results (and vice versa) for both the total field solutions and for individual contributions to the scattered field (Brill and Überall, 1970; Faran, 1951; Harbold and Steinberg, 1968; Neubauer, 1968; Neubauer et al., 1974; Stoyanov et al., 1989). 
This study is an attempt to solve the cylindrical scatterer problem by using the numerical finite difference formulation of the wave equation. Finite difference methods are well established for elastic wave propagation problems concerning both simple and complex structures (Alterman and Loewenthal, 1972; Dougherty and Stephen, 1988 (chapter 2); Frankel and Clayton, 1984; Kelly et al., 1976; Levander and Hill, 1985; Stephen, 1988a,b; Virieux, 1986). The robust nature of the method is not always necessary for acoustics problems or problem with simple geometrical relationships (for which analytical solutions are easily obtained). While various finite difference formulations of the wave equation have been tested against analytical solutions for layered or rectangular structures (Kelly et al., 1976; Stephen, 1983; Virieux, 1986), the more rigorous test of scattering from non-planar, non-rectangular objects has not been well studied. The finite difference formulation used for this study is a 2-D elastic displacement-stress code in rectangular coordinates and has been applied to earth models with complex structures (Dougherty and Stephen, 1988 (chapter 2); Stephen, 1988; Virieux, 1986). The models presented in this work are solved by using the method without any modifications from that used for the earth models. Steady state (using a monochromatic CW source) and transient wave (using a broad-band Gaussian pulse source) solutions are presented. 


\section{CW SOURCE}

The first cylindrical model was run with a monochromatic continuous wavelet (CW) source. The theory for this situation is well developed for a homogeneous elastic infinite cylinder and is briefly outlined below. The geometry of the cylinder and incident $\mathrm{CW}$ plane wave source is shown in figure 4.1 with the cylinder extending infinitely in both directions along the $\mathrm{Z}$ (cylindrical) and/or y (Cartesian) axes and the plane wave extending infinitely in the $\mathrm{x}$ and $\mathrm{y}$ directions (Cartesian). The normal mode solution of Faran (1951) was chosen for the analytical solution because of its relative ease of calculation. The Sommerfeld-Watson transformation technique provides insight into which phases are contributing to the scattered field but is more complicated numerically for small $k a$ values and the total solution is not easily reached. The normal mode solution gives a total scattered pressure wavefield which is easily compared to the total field produced by the finite difference method.

The solution to the infinite elastic cylinder problem consists of solving the wave equation inside and outside of the cylinder and matching solutions at the boundary. The infinite cylinder is independent of the Z-dimension ( into and out of the plane of the page in figure 4.2) and is thus able to be modelled easily by the 2-D finite difference method as well as by the analytical methods. Inside the homogeneous elastic cylinder, displacements are governed by the elastic wave equation for homogeneous media;

$$
(\lambda+2 \mu) \nabla(\nabla \cdot \mathbf{u})-\mu \nabla \times(\nabla \times \mathbf{u})=\rho \frac{\delta^{2} \mathbf{u}}{\delta t^{2}}
$$




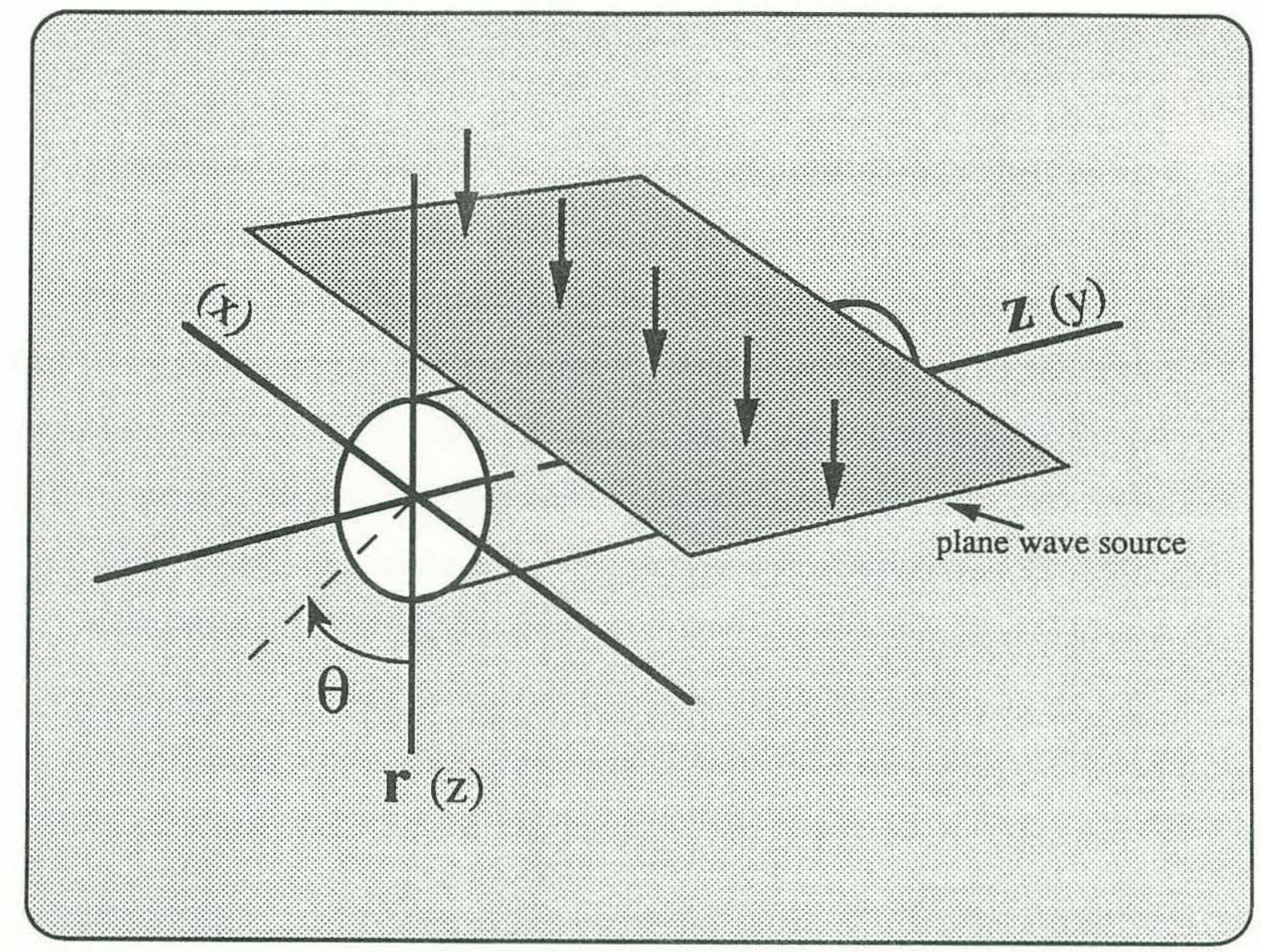

Figure 4.1. Geometrical considerations for the problem of plane wave scattering by an infinite elastic cylinder. Cylindrical coordinates (bold) were used for theoretical formulations and Cartesian coordinates (in parentheses) were used for the finite difference modelling. The cylinder extends infinitely in both directions along the $\mathrm{Z}$ (cylindrical) or $\mathrm{y}$ (Cartesian) axis. 
where $\mathbf{u}$ is displacement, $t$ is time, $\lambda$ and $\mu$ are Lame's constants, $\rho$ is density and $\nabla$ is the del vector operator. The displacement can be derived from a scalar and vector potential:

$$
\mathbf{u}=-\nabla \Psi+\nabla \times \mathbf{A}
$$

where $\nabla \Psi$ is the compressional component of displacement and $\nabla x A$ comprises the shear component within the elastic cylinder. Solutions of wave equations for the potentials within the cylinder involve infinite sum expressions of Bessel functions in cylindrical coordinates;

$$
\Psi=\sum_{n=0}^{\infty} a_{n} J_{n}\left(k_{1} r\right) \cos (n \theta)
$$

$$
A_{z}=\sum_{n=0}^{\infty} b_{n} J_{n}\left(k_{2} r\right) \sin (n \theta)
$$

where $\mathrm{J}_{\mathrm{n}}$ represents the Bessel function of the first kind of order $\mathrm{n}, \mathrm{k} 1,2$ are the compressional and shear wavenumber, respectively, within the solid cylinder and an and $b_{n}$ are coefficients to be determined. Substitution of (3) and (4) into (2) yields the following expressions for radial and azimuthal displacements;

$$
\begin{aligned}
& \mathrm{u}_{\mathrm{r}}=\sum_{\mathrm{n}=0}^{\infty}\left[\frac{\mathrm{nb}}{\mathrm{r}} J_{n}\left(k_{2} r\right)-a_{n} \frac{d J_{n}\left(k_{1} r\right)}{d r}\right] \cos (n \theta) \\
& u_{\theta}=\sum_{n=0}^{\infty}\left[\frac{n a_{n}}{r} J_{n}\left(k_{1} r\right)-b_{n} \frac{d J_{n}\left(k_{2} r\right)}{d r}\right] \sin (n \theta)
\end{aligned}
$$




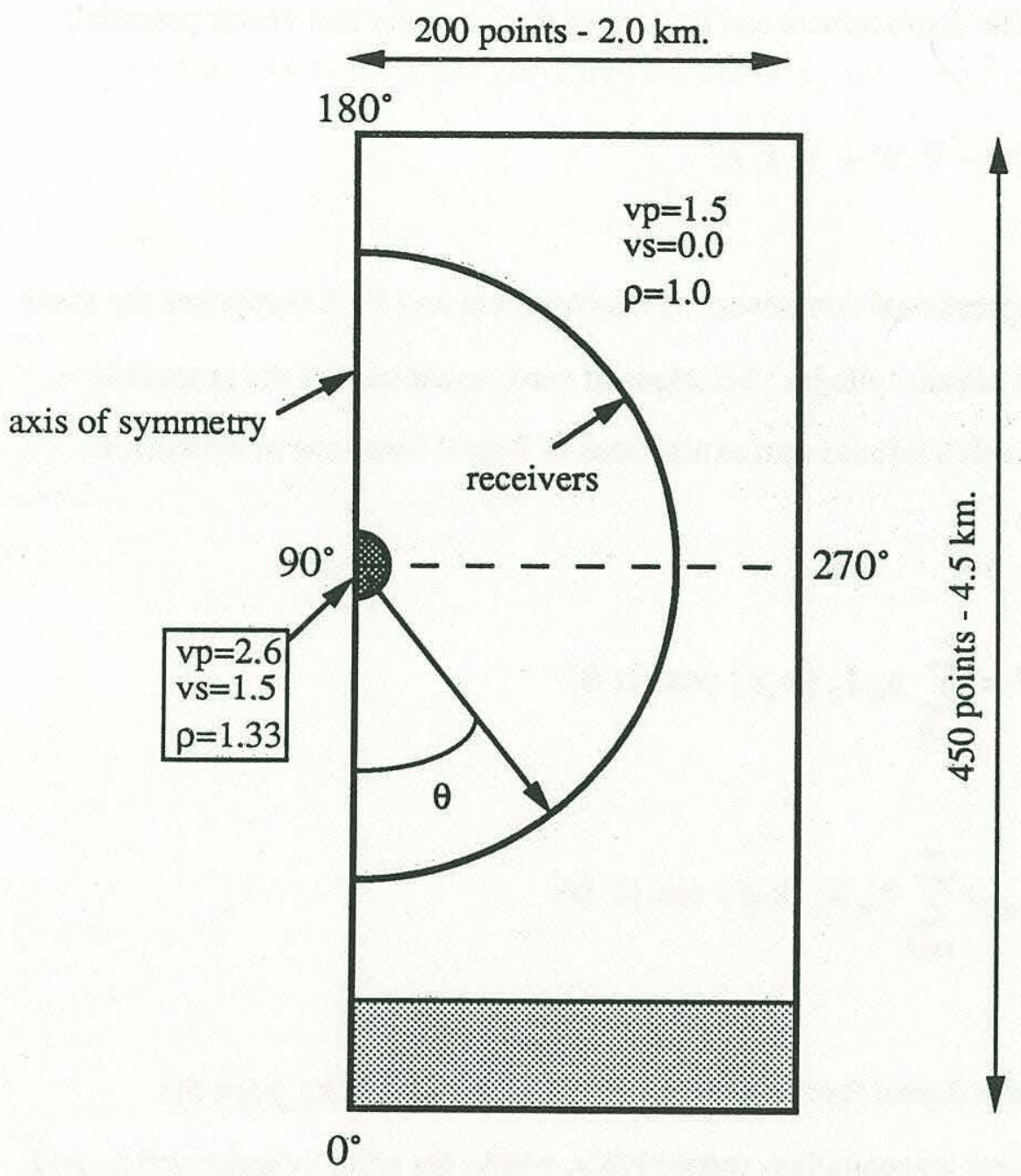

Figure 4.2. Grid geometry used for the finite difference solution. Absorbing boundaries based on the parabolic equation are used on the top and right sides of the grid. The left hand side is an axis of symmetry. Energy travelling downward is attenuated in the stipled region by using the telegraph equation. The cylinder is placed on the axis of symmetry for the CW source model and extends infinitely into and out of the page. 
The field in the fluid outside of the cylinder is somewhat less complicated than that within the elastic medium. Waves within the fluid obey the acoustic wave equation for homogeneous media;

$$
\nabla^{2} \mathrm{p}=\frac{1}{\mathrm{c}_{3}^{2}} \frac{\delta^{2} \mathrm{p}}{\delta \mathrm{t}^{2}}
$$

where $\mathrm{p}$ is pressure and $\mathrm{c} 3$ is the compressional wave velocity in the fluid. The incident $\mathrm{CW}$ plane wave can be represented by;

$$
p_{i}=p_{0} \sum_{n=0}^{\infty} \varepsilon_{n}(-j)^{n} J_{n}\left(k_{3} r\right) \cos (n \theta)
$$

where $p_{0}$ is amplitude , $\varepsilon_{n}$ is the Neumann factor $\left(\varepsilon_{0}=1 ; \varepsilon_{n}=2, n>0\right), j=s q r t(-1)$, and $k_{3}$ is the wavenumber of acoustic waves in the fluid. The radial component of the CW plane wave source is all that is needed to match boundary conditions, it is given by;

$$
u_{i, r}=\frac{p_{0}}{\rho_{3} \omega^{2}} \sum_{n=0}^{\infty} \varepsilon_{n}(-j)^{n} \frac{\delta\left(J_{n}\left(k_{3} r\right) \cos (n \theta)\right)}{\delta r}
$$

Because of symmetry about $\theta=0$, the outgoing scattered pressure and displacement must be given by;

$$
p_{s}=\sum_{n=0}^{\infty} c_{n}\left(J_{n}\left(k_{3} r\right)-j N_{n}\left(k_{3} r\right)\right) \cos (n \theta)
$$


(11) $\quad \mathrm{u}_{\mathrm{s}, \mathrm{r}}=\frac{1}{\rho_{3} \omega^{2}} \sum_{\mathrm{n}=0}^{\infty} \mathrm{c}_{\mathrm{n}} \frac{\delta\left(\left(\mathrm{J}_{\mathrm{n}}\left(\mathrm{k}_{3} \mathrm{r}\right)-\mathrm{j} \mathrm{N} \mathrm{N}_{\mathrm{n}}\left(\mathrm{k}_{3} \mathrm{r}\right)\right) \cos (\mathrm{n} \theta)\right)}{\delta \mathrm{r}}$

where $\mathrm{N}_{\mathrm{n}}$ is the Bessel function of the second kind of order $\mathrm{n}$ and $\mathrm{c}_{\mathrm{n}}$ are the coefficients needed to describe the scattered pressure field. These coefficients are found by matching boundary conditions across the cylinder surface. At a liquid-solid interface there are three conditions which must be met;

i. continuity of normal stress (in the fluid, normal stress is given by the total pressure);

$$
\mathrm{p}_{\mathrm{i}}+\mathrm{p}_{\mathrm{s}}=-\tau_{\mathrm{r} 1}
$$

ii. continuity of the normal component of displacement;

$$
\mathrm{u}_{\mathrm{i}, \mathrm{r}}+\mathrm{u}_{\mathrm{s}, \mathrm{r}}=\mathrm{u}_{\mathrm{r}}
$$

iii. tangential stress within the cylinder must vanish at the interface (liquid will not support shear);

$$
\tau_{\mathrm{r}, \theta}=\tau_{\mathrm{r}, \mathrm{z}}=0
$$

Solving these simultaneous equations for $\mathrm{p}_{\mathrm{s}}$ is complicated because of the infinite series involved and the number of constants $\left(c_{n}\right)$ which must be determined. This has been done by Faran (1951) who arrived at an expression for the amplitude of scattered pressure in the far field as a function of azimuth; 


$$
\left|p_{s}\right|=p_{0}\left(\frac{2}{\pi k_{3} r}\right)^{1 / 2}\left|\sum_{n=0}^{\infty} \varepsilon_{n} \sin \eta_{n} \exp \left(j \eta_{n}\right) \cos (n \theta)\right|
$$

The expressions for the scattering phase angles, $\eta$, are too involved for presentation here but are given by Faran (1951) and Lowan et al. (1946) and are composed of complex expressions of Bessel functions and their derivatives.

Equation 15 (including the expressions for $\eta$ ) was solved out to 15 terms for comparison with the finite difference runs discussed below. Although this modal solution is analytical, it must be numerically evaluated. In order to test the accuracy of the numerical formulation of the analytical solution used for this work, the results of the code were compared against Faran's results for metal cylinders with excellent agreement. Also, values output by the numerical formulation for the intermediate scattering angles, $\eta$, were tested against tables in Faran (1951) and Lowan et al. (1946), again with excellent agreement.

The velocities and densities of metal cylinders presented in most of the literature are too high to be realistic for earth materials in the upper crust. The metal cylinders commonly used for test experiments have relatively large elastic constants ( a p-wave velocity of 4.28 $\mathrm{km} / \mathrm{sec}$, shear wave velocity of $2.165 \mathrm{~km} / \mathrm{sec}$ and a density of $8.5 \mathrm{gm} / \mathrm{cc}$ for brass). These high values represent a velocity contrast of over 200 per cent at the water-solid interface and are cause instabilities in the finite difference modeling scheme used here. The oceanic crustal scattering models presented in Dougherty and Stephen (1988) and heterogeneous crustal halfspace models presented by Frankel and Clayton (1984) have a maximum crustal velocity variation of 10 per cent. Since this study is an attempt to verify the use of the finite difference formulation for scattering in the upper crust, a much smaller velocity contrast 
was chosen for the infinite cylinders used in this work. Because most effects need a fairly significant contrast to be visible, a p-wave velocity of $2.6 \mathrm{~km} / \mathrm{sec}$, s-wave velocity of 1.5 $\mathrm{km} / \mathrm{sec}$, and density of $1.33 \mathrm{gm} / \mathrm{cc}$ were chosen as the parameters for the cylinder. These elastic parameters provide enough contrast to show effects but not so much that it is unrealistic for the earth models or that instabilities in the formulation will be developed.

The orientation of the cylinder on the finite difference grid is shown in figure 4.2. A radius of 160 meters was used to give a $k a$ value (wavenumber, $\mathrm{k}$, times radius, a) of 6.7 for a $10 \mathrm{~Hz}$ source. An attempt was made to use the same finite difference formulation and grid setup (including the same boundary conditions, transition zone setup, etc) as was used for the earth models (Dougherty and Stephen, 1988 (chapter 2), and chapter 3). While this may not be the optimal setup for solving the cylinder problem, we did not feel that it was necessary or valid to create special conditions for this problem. The cylinder was placed on the axis of symmetry in order to save computer costs. Since this is theoretically a symmetrical problem (with a symmetrical solution), it is not necessary to calculate both sides of the cylinder. Some initial models were run which calculated the entire cylinder to check the feasibility of putting the cylinder on the axis of symmetry. These results indicated an inherent asymmetry in the way the grid was being defined. This problem was corrected and the finite difference models were indeed made to be symmetric.

Receivers are located in a semi-circle surrounding the center of the cylinder at a distances of 1.4 kilometers. Azimuthal distribution of receivers is one per degree. A 10 $\mathrm{Hz}$ sinusoidal CW source was introduced as a plane wave along the top of the grid. The source has only a vertical component of displacement so that the solution is symmetric about the center of the cylinder (range $=0$ ). Other details of the grid such as source introduction, absorbing boundaries, etc. can be found in Dougherty and Stephen (1988). 
The CW finite difference model used 10 meter grid spacing and a time step of 1 millisecond. These grid and time step dimensions are well within the accepted guidelines for numerical stability and dispersion requirements of the grid formulation (Kelly et al., 1976; Virieux, 1986). The length of the run (6 seconds or 6000 timesteps) was long enough for steady state to be reached.

The quantity of interest from the finite difference runs is the scattered pressure field. Since the total field is the default output of the method, the unperturbed source field must be subtracted to leave the scattered field. This was done by computing an acoustic halfspace model (no cylindrical scatterer) with receivers in the same positions as in the scattering model. Then, the total and unperturbed finite difference fields are both known and the scattered field can be obtained by a simple subtraction. The time series of the scattered field for the CW model are shown in figure 4.3. This is the scattered pressure in the water at a distance of 1.4 kilometers from the center of the cylinder. The time series are plotted against azimuths from 180 degrees (backscattering direction) to 360 degrees (full forward scattering direction). Scattering angles from 1 to 179 degrees have symmetric counterparts in the series presented.

The time series demonstrate a number of features unique to the scattering from a $\mathrm{CW}$ source. First, the transition to steady state takes place about 2.0 seconds after the beginning of the scattered signal (or about 4.25 seconds into the time series). Most of the time series go through some amplitude fluctuations until about 4.25 seconds when the amplitude levels out. Since the CW source is monochromatic, the time series also contain a single frequency for all azimuths and times. Subsequent to the onset of steady state, the only quantity in the time series which is variable is the pressure amplitude as a function of azimuth. Because of the elastic nature of the cylinder and the existence of circumferential waves (in the full wave solution of the finite difference method) there is no geometric 
Figure 4.3. Time series of scattered pressure from the $10 \mathrm{~Hz} \mathrm{CW}$ source for circular receivers between 180 and 360 degrees (see figure 4.2) at a distance of $1.4 \mathrm{~km}$. from the cylinder center. These data show a strong dependence of scattering strength on azimuth. 


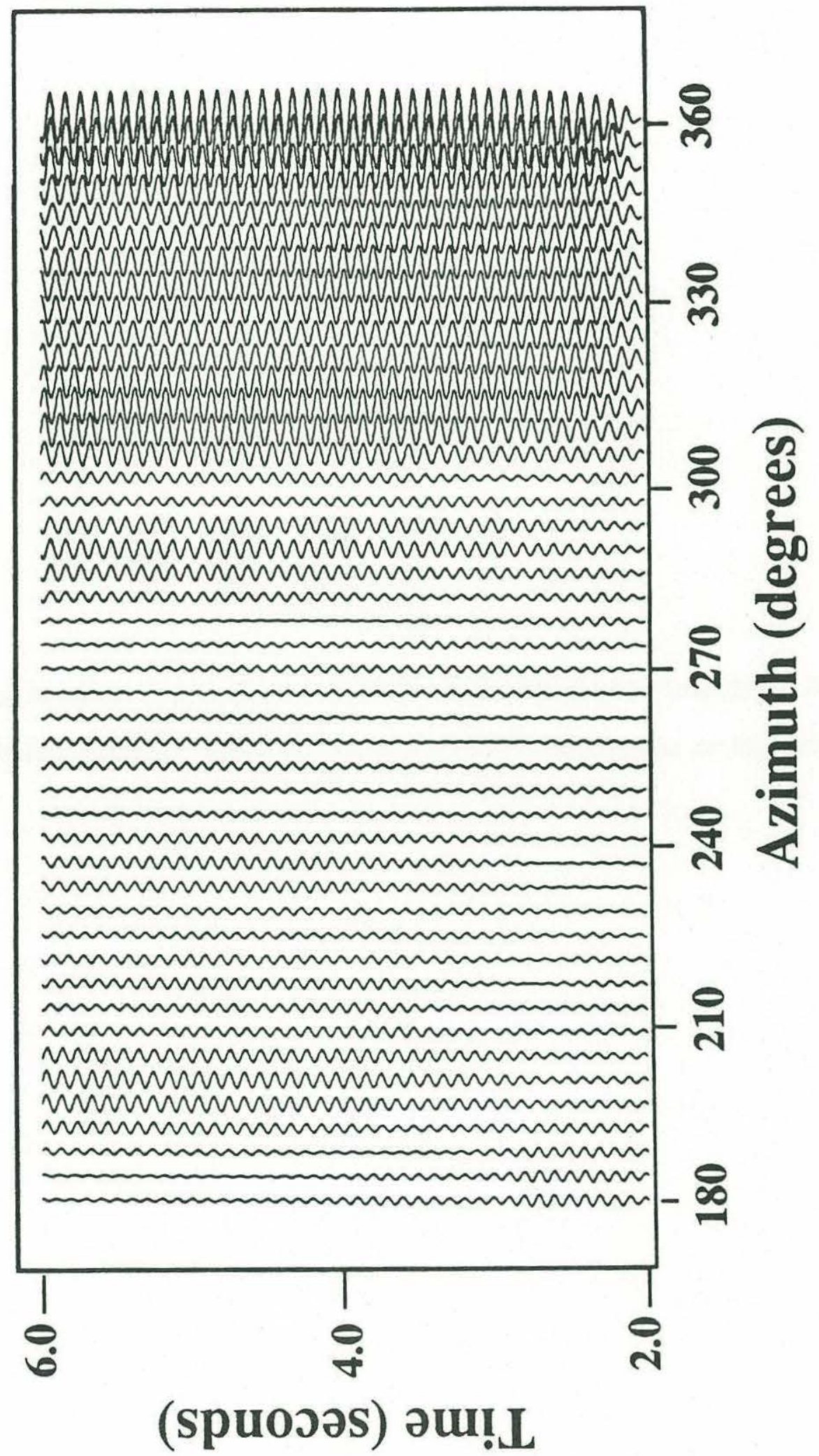


Figure 4.4. Analytical (solid line) vs. finite difference (dashed line) results of scattered pressure strength vs. azimuth for the $10 \mathrm{~Hz} \mathrm{CW}$ source model. Cylinder radius is 0.160 $\mathrm{km}$. 


\section{Scattering pattern}

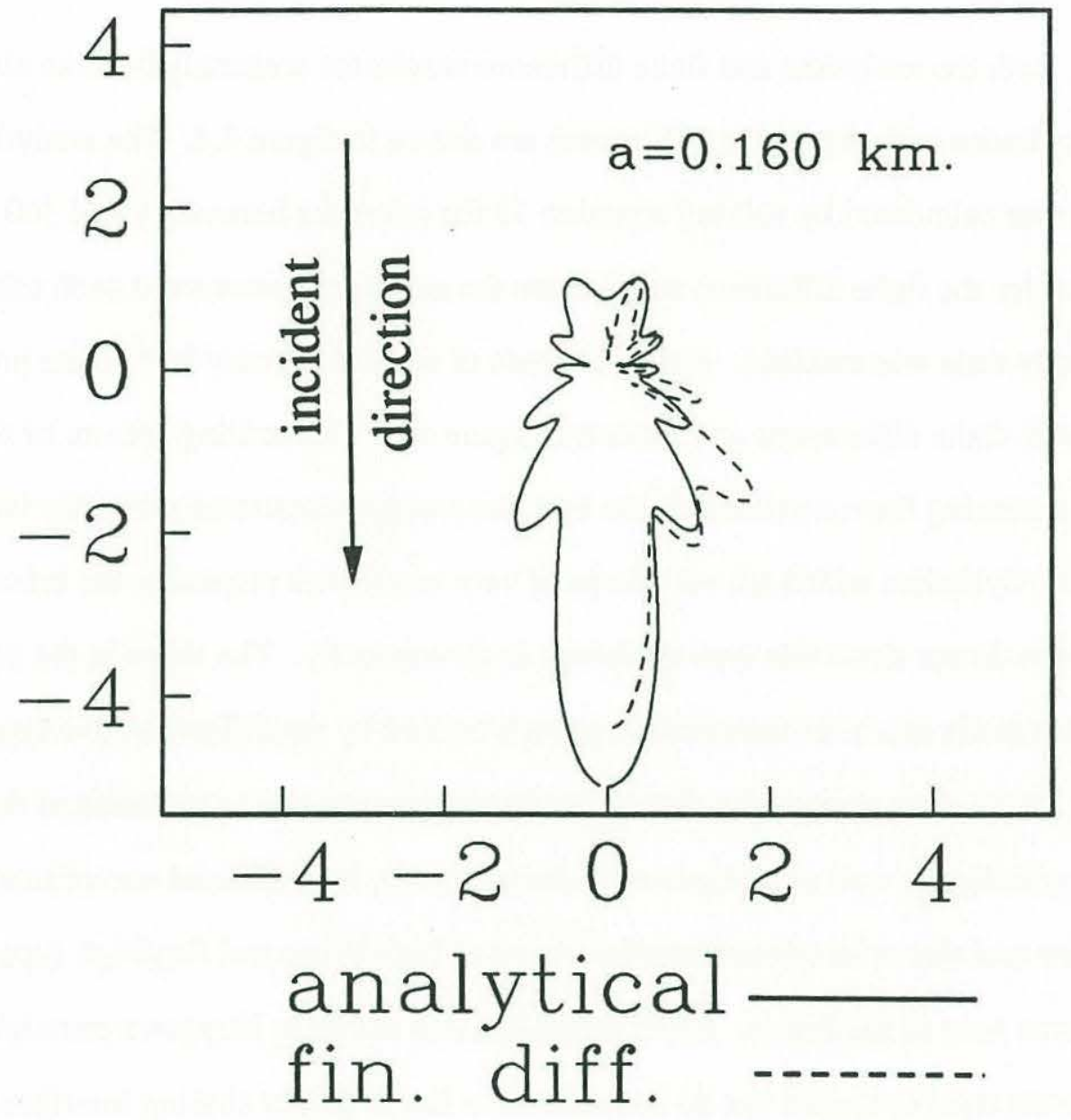


shadow zone in the forward direction. In fact, the strongest signal occurs in forward directions and smaller amplitudes occur at a number of azimuths within the figure. This is also predicted by the normal mode analytical solution.

Both the analytical and finite difference results for scattering from an elastic infinite cylinder with the $10 \mathrm{~Hz} \mathrm{CW}$ source are shown in figure 4.4. The analytical solution was calculated by solving equation 15 for azimuths between 1 and 360 degrees. The value for the finite difference solution are the maximum pressure at each azimuth taken after steady state was reached. Again, because of symmetry, only half of the problem was solved with finite differences and plotted in figure 4.4. The striking feature of the pattern for CW scattering from a cylinder is the fact that energy is scattered primarily into distinct azimuths. Cylinders which are very large or very small with respect to the seismic wavelength do not show this type of pattern as dramatically. The lobes in the scattering pattern are really due to an interference pattern created by the different wave types of the system. Body wave such as the direct plane wave source, waves transmitted through the elastic cylinder (as well as whispering gallery modes), and reflected waves interfere with each other and also with circumferential waves of both Franz and Rayleigh types to create the patterns seen in the figure. Franz waves are due solely to Huygen's principle and exist for perfectly rigid cylinders but do not exist for a flat or gently sloping interface (without shadow zones). Rayleigh-type circumferential waves exist only with elastic media and degenerate to true Rayleigh waves for flat interfaces.

The agreement between the analytical and finite difference solutions is quite good considering the coarseness of the grid used and the fact that a rectangular rather than cylindrical coordinate system is used for the finite difference formulation. A curved surface, such as the edge of the cylinder, defined on a rectangular grid must necessarily be 
stepwise. Previous work (chapter 3) has shown that scattering does occur from these small steps which will affect the interference pattern seen in figure 4.4.

The finite difference method also does some inherent averaging over space (and time ) in calculating spatial derivatives. This averaging can blur the actual location of boundaries. The interference pattern seen in a plot of scattered pressure vs. azimuth is very sensitive to the diameter of the cylinder for a given wavelength of energy. Analytical scattering patterns for cylinders with radii of one grid step more and less $(0.17$ and 0.15 $\mathrm{km}$.) and one half grid step more and less $(0.165$ and $0.155 \mathrm{~km})$ that that used for the finite difference model are shown in figure 4.5. Small changes in diameter (or $k a$ ) have a dramatic influence on the scattering pattern and the averaging done by the method probably does account for at least some of the discrepancies between the analytical and finite difference solutions, especially for azimuths near the backscattered direction (90<azimuth<270).

It is extremely difficult, if not impossible, to pick contributions from the individual wave types in the time series or scattering patterns. A few of the individual contributions can, however, be seen in the snapshots of compressional energy on the grid. Figure 4.6 is the snapshot of compressional and shear scattered (unperturbed source field removed) energy on the grid 4.0 seconds after introduction of the CW source onto the top of the grid. Normalized compressional and shear energy was calculated by using the divergence and curl of displacements output by the finite difference formulation (see Dougherty and Stephen, 1988 (chapter 2)). The scattered field was obtained from the total field by the same procedure used for the time series of subtracting the homogeneous halfspace solution from the total field to leave the scattered field. 
Figure 4.5. Analytical (solid line) vs. finite difference (dashed line) results of scattered pressure strength vs. azimuth for the $10 \mathrm{~Hz} \mathrm{CW}$ source model. Cylinder radii of $0.15 \mathrm{~km}$ (4.5a.), $0.155 \mathrm{~km}$. (4.5b.), $0.165 \mathrm{~km}$. (4.5c.), and $0.170 \mathrm{~km}$. (4.5d.) for the analytical patterns. The finite difference results are for a cylinder radius of $0.16 \mathrm{~km}$. The analytical pattern is especially sensitive to cylinder radius in the backscattered direction. 

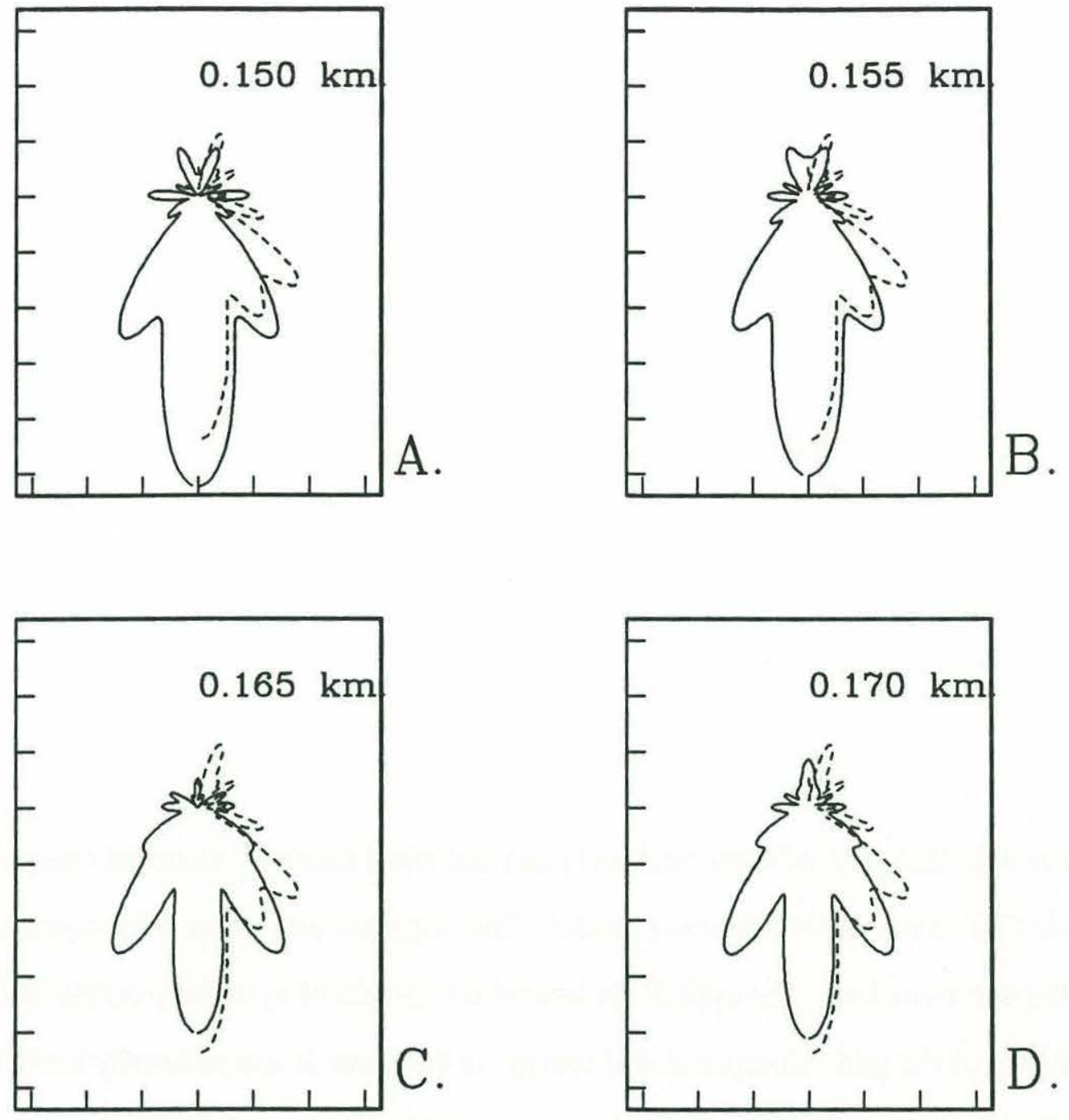
Figure 4.6. Snapshot of compressional (top) and shear (bottom) scattered energy for the $10 \mathrm{~Hz} \mathrm{CW}$ source finite difference model. This snapshot was taken 4.0 seconds after the source was turned on. The cylinder is located on the axis of symmetry on the left hand boundary of the grid. Compressional energy in the water is scattered only into a few discrete directions. Shear energy only appears inside of the elastic cylinder. 


\section{CW SCATTERED ENERGY}
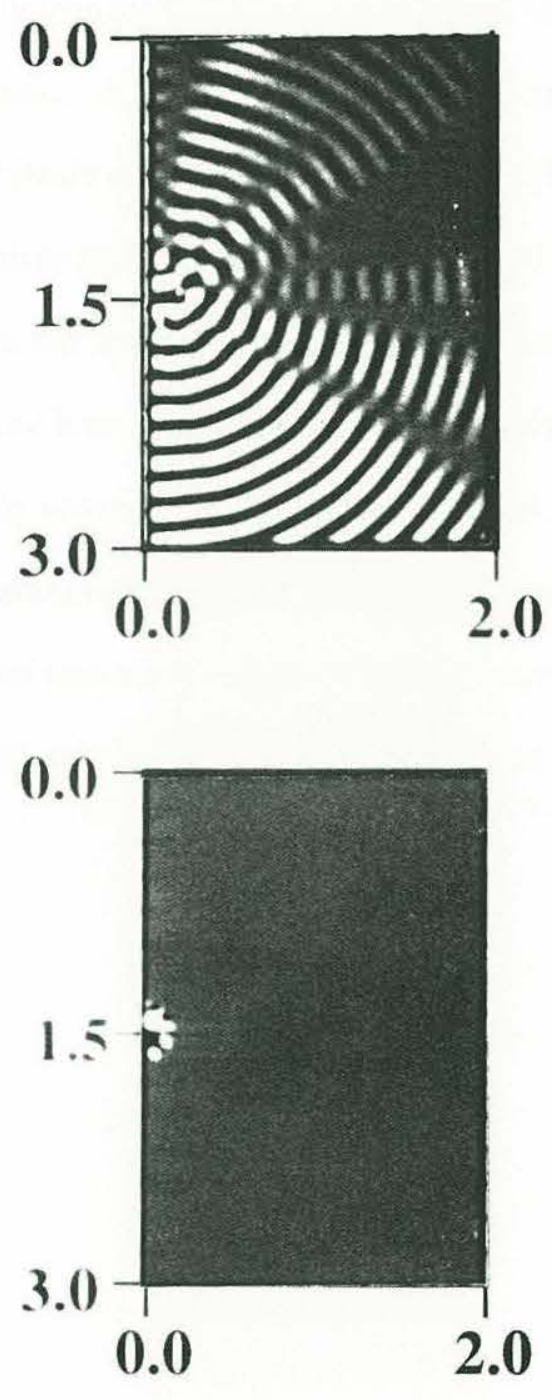
The main scattering features which can be seen in the snapshots of figure 4.6 are the interference pattern and the energy inside the cylinder. The interference pattern in the fluid manifests itself as energy scattered into certain discrete azimuths with the main lobe being in the forward direction (no 'shadow zone'). Also, at distances greater than about 3 cylinder diameters, the scattered field does not change significantly with increased distance from the cylinder center (along a particular azimuth). Two clear contributors to the scattered interference pattern in the fluid can be seen within the elastic cylinder and along its boundary. Body waves transmitted into the cylinder can be seen inside the elastic solid (refer to figure 4.2 for cylinder boundaries). Although much of the transmitted energy probably is reverberated as 'whispering gallery' modes, direct and vertically travelling compressional and shear waves can be seen in the upper and lower plots respectively. These waves interfere directly with circumferential waves both inside and outside of the cylinder boundary. Elastic components of the interface waves can be seen along the inside of the cylinder boundary especially in the shear energy plot (lower part of figure 4.6). 


\section{PULSE SOURCE}

Another method which can be used to examine scattering from an elastic cylinder is to use a broad band pulse source rather than a monochromatic CW source. Although the scattering patterns presented in figures 4.4 and 4.5 are useful, they only provide the response for a single frequency. Another quantity, $\mathrm{F}_{\infty}$, the scattering form function, provides the strength of scattering in any direction for all frequencies present in the broad band source. Each monochromatic $\mathrm{CW}$ solution contains one value of $\mathrm{F}_{\infty}$ at a particular value of $k a$. By using a broad band pulse source, a range of values of $F_{\infty}$ can be established in a single experiment or finite difference run. In this way, a pattern of the influence of frequency (or conversely, diameter of the cylinder for a single frequency) on the scattering strength can be determined. Since it is known that scattering strength, especially in the backscattering direction, is very sensitive to the diameter of the cylinder (see figures 4.4 and 4.5 ) perhaps a better test of the finite difference method is to examine the pattern of the backscattering form function rather than try to precisely match a particular monochromatic response.

A time domain solution to the transient source problem can also give added insight to the mechanisms of scattering which result in the interference patterns of the monochromatic source problem. The short duration of the pulse allows the wavefield to separate into discrete components after initial interaction with the cylinder. Time series for both the analytical and finite difference solutions can be obtained and compared for the existence and location of arrivals in time. Successive snapshots of wavefronts show quite clearly how the incident energy is partitioned between direct, transmitted, and circumferential phases. Another advantage of using the pulse source is that steady state 
need not be obtained for the analysis (although sufficient time must be run to include all of the significant arrivals).

Theoretically, the use of a broadband pulse source to determine the amplitude of the backscattering form function is straight forward and follows the work of (Dardy et al., 1977; Neubauer, 1986). The plane wave pressure source used for the finite difference models is in the shape of the second derivative of a Gaussian curve and is given by (Kelly et al., 1976; Stephen, 1985);

$$
\mathrm{p}_{\mathrm{i}}(\mathrm{t})=\mathrm{A}\left[4 \xi\left(3 \mathrm{t}-2 \xi \mathrm{t}^{3}\right) \exp \left(-\xi \mathrm{t}^{2}\right)\right]
$$

and the Fourier transform is given by;

$$
g_{i}(k a)=\int_{-\infty}^{\infty} p_{i}(t) \exp (-j \omega t)
$$

The parameter $\xi$ in equation (16) determines the peak frequency and bandwidth of the source wavelet. Both the source time function and its Fourier transform are given in figure 4.7. This wavelet has a peak frequency of $10 \mathrm{~Hz}$ and upper and lower halfpower frequencies of 13.5 and $6.8 \mathrm{~Hz}$ respectively. The peak, upper, and lower frequencies correspond to $k a$ values of $6.7,9.0$, and 4.5 respectively.

The form function for a given receiver azimuth relates incident to scattered pressure for a range of $k a$ values. From equation (15), the scattered pressure in the far field for a given azimuth is; 


$$
p_{i}(\theta)=p_{0}\left(\frac{2}{\pi k_{3} r}\right)^{1 / 2} \sum_{n=0}^{\infty} \varepsilon_{n} \sin \eta_{n} \exp \left(j \eta_{n}\right) \cos (n \theta)
$$

and the form function for the backscattered direction is given by;

$$
f_{\infty}(\pi)=\left(\frac{\pi k_{3} r}{2}\right)^{1 / 2}\left(\frac{p_{s}(\pi)}{p_{0}}\right) \sum_{n=0}^{\infty} \varepsilon_{n} \sin \eta_{n} \exp \left(j \eta_{n}\right) \cos (n \theta)
$$

The backscattered pressure has the Fourier transform;

$$
g_{s}(k a, \pi)=\left(\frac{2}{\pi k_{3} r}\right)^{1 / 2} f_{\infty}(k a, \pi) g_{i}(k a)
$$

so that the amplitude of the backscattered form function can be expressed as;

$$
\left|f_{\infty}(k a, \pi)\right|=\left(\frac{\pi k_{3} r}{2}\right)^{1 / 2}\left|\frac{g_{s}(k a, \pi)}{g_{i}(k a)}\right|
$$

The finite difference method uses a pressure source function given by equation (10) and calculates scattered pressure (equation 18). After calculation of the Fourier transforms of these two pressures (equations 17 and 20) the amplitude of the backscattered form function with respect to $k a$ can be determined by equation 21 . The analytical solution to scattering of the source function given in equation 16 by an aluminum cylinder can be obtained by equation (15). Calculation of the backscattered form function for this case by using equation (21) agrees exactly with the analytical curve for an aluminum cylinder seen in Dardy et al (1977, figure 3). 
Figure 4.7. Frequency content (4.7a.) and pressure time signal (4.7b.) of the Gaussian pulse source used for the transient problem. 

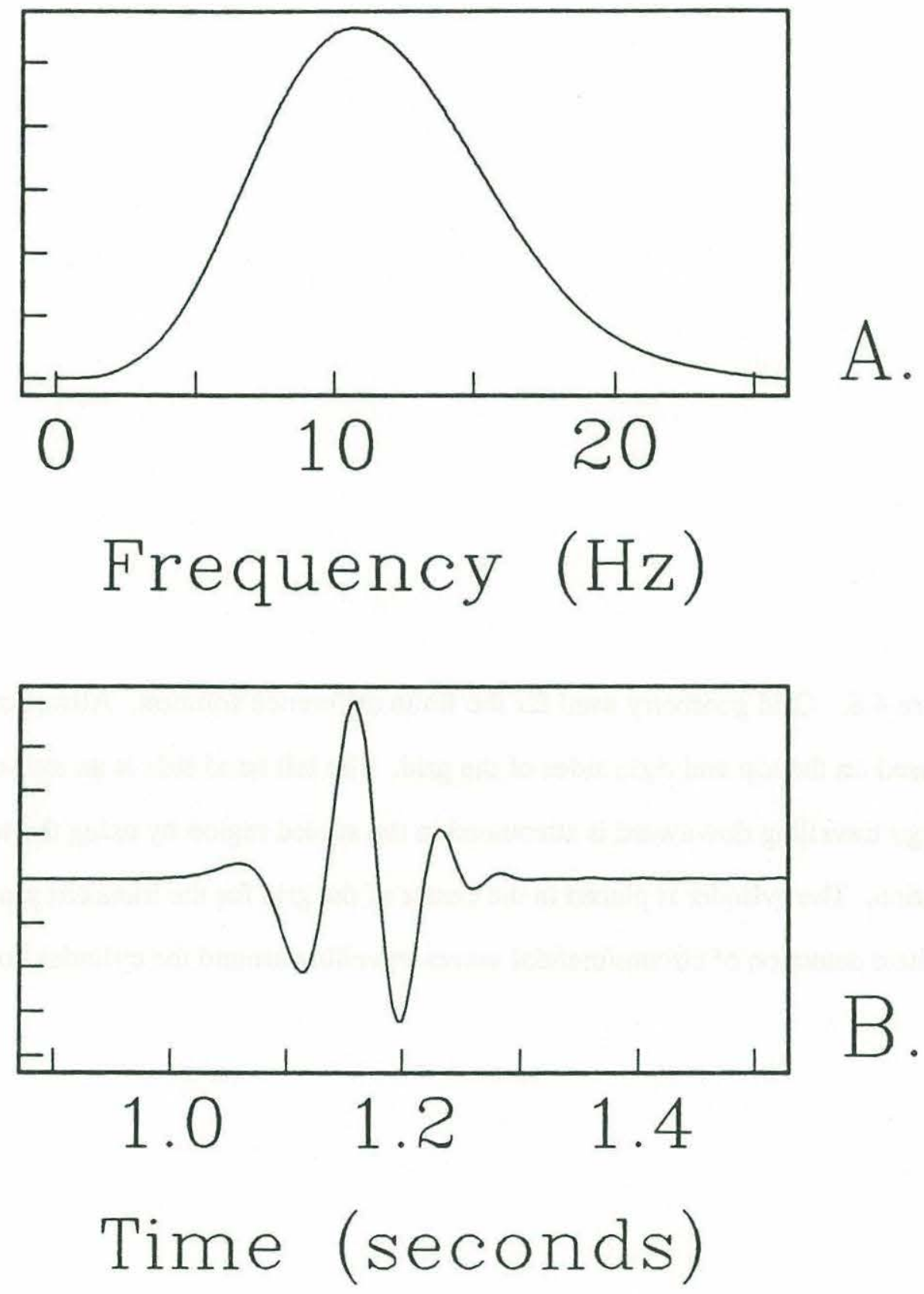
Figure 4.8. Grid geometry used for the finite difference solution. Absorbing boundaries are used on the top and right sides of the grid. The left hand side is an axis of symmetry. Energy travelling downward is attenuated in the stipled region by using the telegraph equation. The cylinder is placed in the center of the grid for the transient problem to facilitate detection of circumferential waves travelling around the cylinder boundaries. 


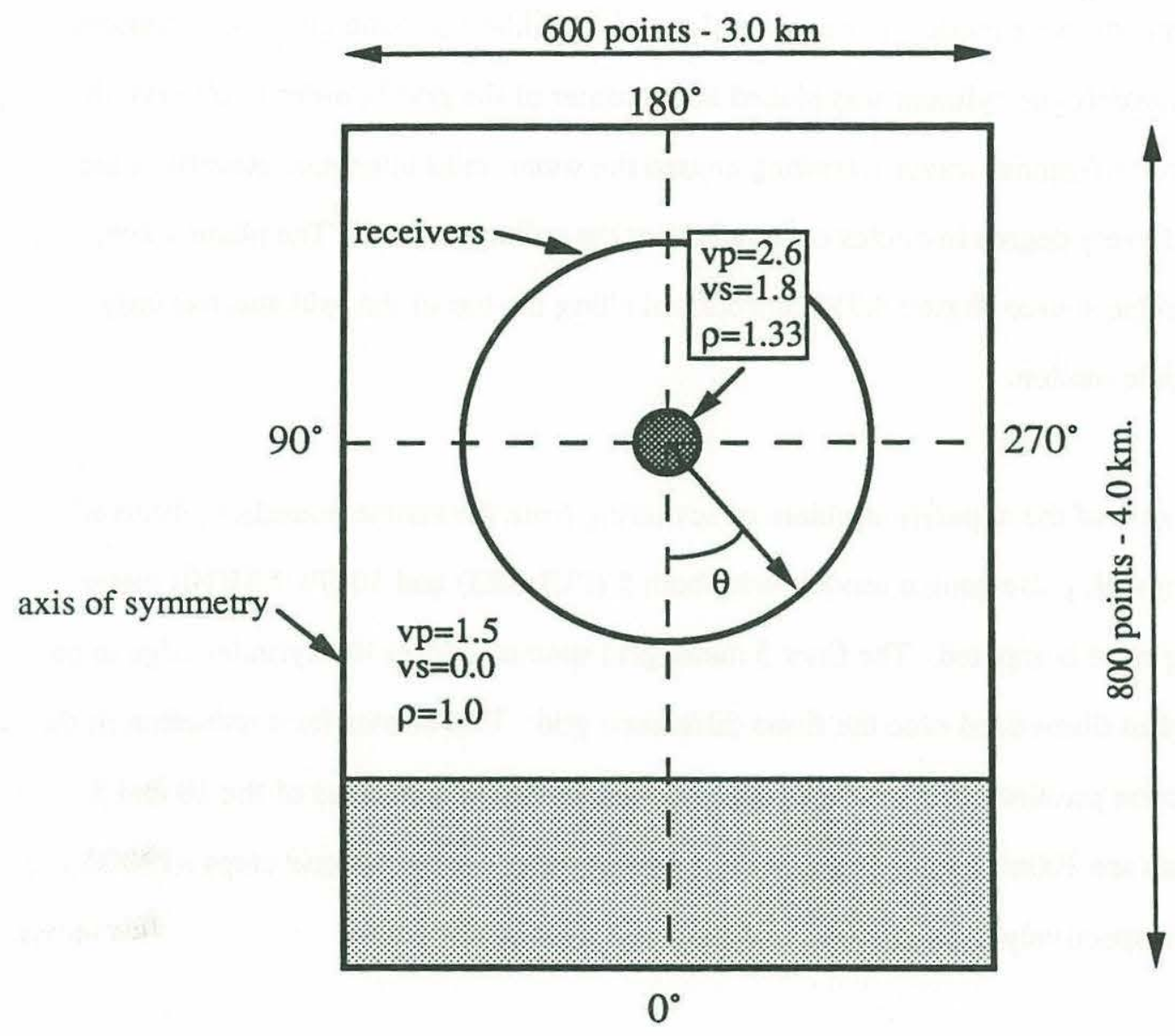


The cylinder model was rerun with a few slight modifications for the pulse source. The geometry for these models is shown in figure 4.8. Although more computer memory and time is needed, the cylinder was placed at the center of the grid in order to observe the predicted circumferential waves travelling around the water-solid interface. Receivers are again placed every degree in circles centered about the cylinder center. The plane wave, broadband pulse source (figure 4.7) is introduced along the top of the grid and has only vertical particle motion.

Because of the apparent problem of scattering from the coarse boundary observed in the CW model, pulse source models with both 5 (PULSE5) and 10 (PULSE10) meter grid spacing were computed. The finer 5 meter grid spacing causes the cylinder edge to be smoother when discretized onto the finite difference grid. This allows for a reduction in the edge diffraction problem of the coarser models. The overall dimensions of the 10 and 5 meter models are $300 \times 400$ grid steps $\times 6000$ times steps and $600 \times 800$ grid steps $\times 12000$ time steps, respectively.

Snapshots of pressure determined by the finite difference method are shown in figure 4.9 for model PULSE5. The time domain solution provided by the method gives added insight into the wavefronts which contribute to the problem which is not available with the CW source models. The partitioning of energy from the pulse is readily visible in the snapshots. Snapshots in figure 4.9 are shown for every 0.15 seconds starting 0.5 seconds after the plane wave source is introduced along the top of the grid. In figure 4.9a, the plane wave source has just reached the cylinder. Elastic wave velocities within the cylinder (p-wave velocity of $2.6 \mathrm{~km} / \mathrm{sec}$, s-wave velocity of $1.5 \mathrm{~km} / \mathrm{sec}$ ) cause the portion of the wavefront transmitted into the cylinder to move ahead of the source wave and increase in wavelength. 
The scattered wavefield becomes much more complicated after the source wave has passed completely through the region of the cylinder (figures $4.9 \mathrm{~b}-\mathrm{f}$ ). The circular wavefronts which emanate from the cylinder in later time steps are complex combinations of reflected, transmitted, and circumferential waves predicted by various ray and acoustic theoretical studies.

In the backscattered direction (towards the top of each snapshot in figure 4.9), the strongest and most obvious scattered arrival is the reflected wave seen in all but the first snapshot of figure 4.9. Energy which is transmitted once through the cylinder makes up the strongest arrivals in the forward scattering directions. Most of the important wavefronts which combine in the $\mathrm{CW}$ case to form the complex interference patterns of figures 4.4 and 4.5 are present in figure $4.9 \mathrm{~d}$. The reflected $(R)$ and transmitted $(T)$ waves are results of simple ray theoretical interactions with the cylinder boundaries. Fast (FI) and slow (SI) interface or circumferential waves are due to the curved nature of the interface. The slow interface wave is similar to the Franz wave present in the rigid cylinder case (Doolittle and Überall, 1966; Frisk et al., 1975; Karal and Keller, 1964). In this elastic case, however, it does have an elastic wave component inside the cylinder. This circumferential wave has a cardioid shape in the fluid portion of the model and shows up best in figures $4.9 \mathrm{~d}$ and $4.9 \mathrm{e}$. Whispering gallery modes and the interference of fast interface waves make up the high energy pulse travelling in the backscattered direction just inside of the reflected wavefront (seen in figure $4.9 \mathrm{c}, \mathrm{d}, \mathrm{f}$ ). Other energy in the snapshots is due to continuous leaking of whispering gallery modes back into the fluid and scattering of these modes and circumferential waves from the corners present on the discretized cylinder boundary. 
Figure 4.9. Snapshots of compressional energy in the finite difference grid of the pulse source model PULSE5. See figure 4.8 for the location of the cylinder in the center of the grid. Cylinder radius is $0.16 \mathrm{~km}$. with grid spacing of 5 meters. The snapshot time increment is 0.15 seconds. In the first frame (4.9a.), the source has just illuminated the cylinder causing a backscattered reflection and a transmitted wavefront which is slightly ahead of the source plane wave because of the faster P-wave velocity in the cylinder. 0.75 seconds later (4.9f.), the scattered field is comprised of a complex combination of reflected and diffracted waves, whispering gallery reverberations, and circumferential waves (cardioid-shaped wavefront in figure 4.9f.). 
PULSE5 SCATTERED PRESSURE
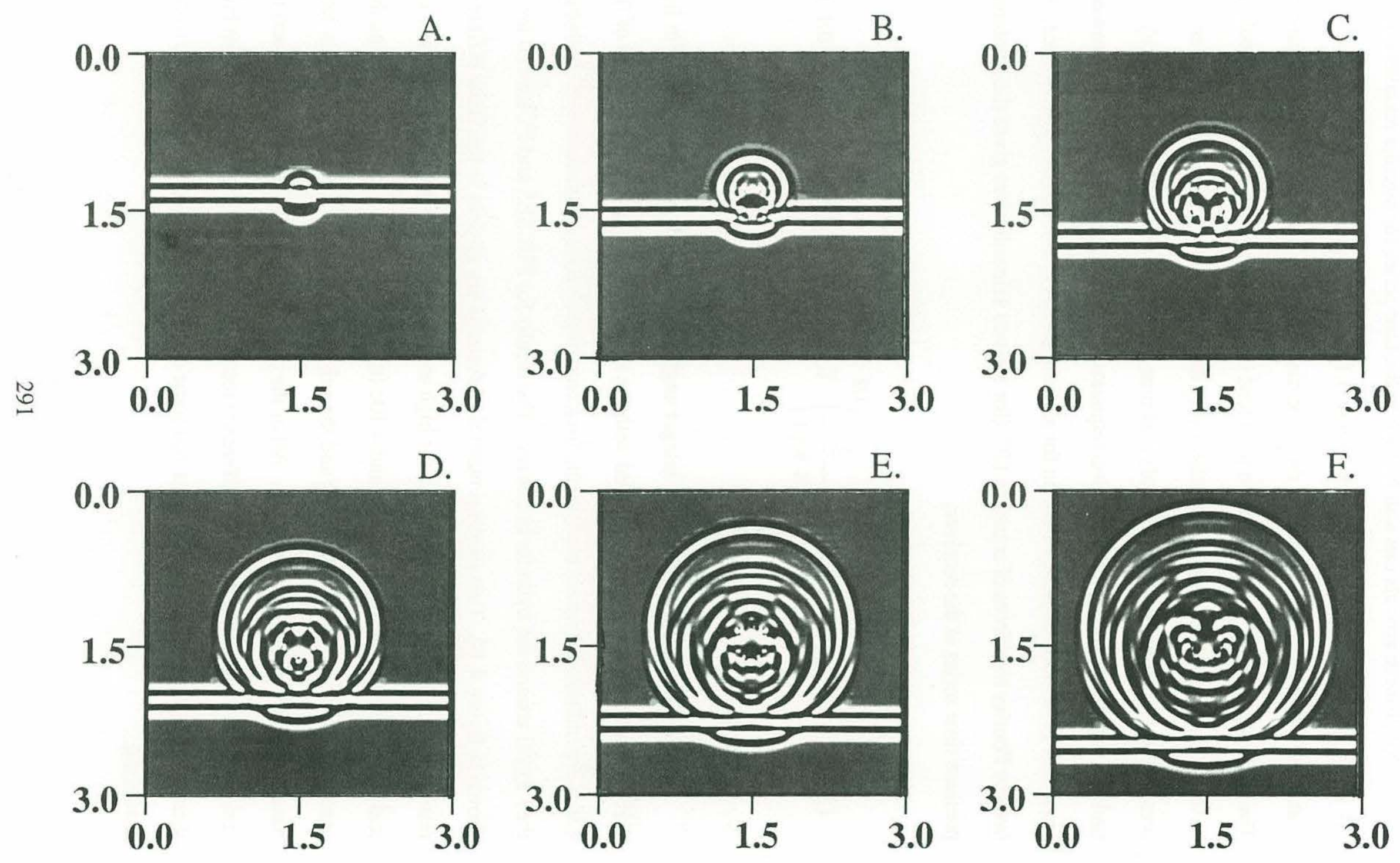
All of these arrivals can also be seen in the time series for circular receivers located $1.4 \mathrm{~km}$.from the center of the cylinder (figure 4.10). Time series traces can also be obtained analytically for receivers at the same discrete points and are shown in figure 4.10. Theoretically, snapshots such as those produced by the finite difference method could also be calculated analytically but would take a very large amount of computer time given present computational resources. Analytical time series are created for each angle by multiplying the scattering form function (equation 19 at any azimuth) and source power (equation 17) in the frequency domain for all frequencies present in the pulse source. An inverse Fourier transform of equation (20) (for a given azimuth) then gives the scattered pressure time series at the receiver;

$$
p_{s}(t, \theta)=\int\left(\frac{2}{\pi k_{3} r}\right)^{1 / 2} f_{\infty}(k a, \pi) g_{i}(k a) \exp (-j \omega t) \partial \omega
$$

Equation (22) was solved for integer angles up to 360 degrees and plotted in figure 4.10a. Time series arrivals are marked with the same notation as in the snapshots of figure 4.9. Circumferential waves cross in the backscattered direction (azimuth $=180$ degrees) as they travel around the cylinder boundary. The results for PULSE5 and PULSE10 are also shown in figure 4.10. Two obvious numerical features are present in the finite difference results and can be ignored. These are the high amplitude source arrivals in the lower rught and left hand sides of figures $4.10 \mathrm{~b}$ and $4.10 \mathrm{c}$ (the analytical solution only contains the scattered pressure, not the total field) and the axis of symmetry arrivals in the upper left hand side of figures $4.10 \mathrm{~b}$ and $4.10 \mathrm{c}$. All of the arrivals expected from the analytical time series are also present in the finite difference results. PULSE5 has good agreement but there are discontinuities in the arrivals and some additional incoherent arrivals due to edge scattering. 
As mentioned earlier, the backscattered form function is often used to describe scattering from a cylinder. This is obtained analytically by using equation 21 . The finite difference value for this is derived from the signal of the receiver at the backscattered direction with the source pulse removed. The procedure used is essentially the inverse of the procedure used for creating the analytical time series but with the finite difference scattered pressure in the left hand side of equation 22 . These functions for the analytical and PULSE5 models are shown in figure 4.11 for $k a$ values within the band of the source frequencies. Shown in the figure are analytical (solid line) traces assuming cylinder radii of $0.16 \mathrm{~km}, 0.165 \mathrm{~km}$. and $0.17 \mathrm{~km}$. (figures $4.11 \mathrm{a}, 4.11 \mathrm{~b}, 4.11 \mathrm{c}$, respectively) compared with the finite difference solution for a cylinder radius of $0.16 \mathrm{~km}$. (dashed lines in figures 4.11a, 4.11b, 4.11c). The shapes of the curves for the finite difference model do not change noticeably but the different cylinder radii values have the effect of a phase shift on the curves. The best match for the curves is found for a cylinder radius of $0.17 \mathrm{~km}$. Apparently, the finite difference source wave is 'seeing' a slightly larger cylinder than is actually being used. In all cases, the absolute level of the backscattered form function curve is comparable between the analytical and finite difference solutions. Considering the sensitivity of the backscattered pressure to cylinder radius seen in figure 4.4 and 4.5 , the finite difference approximation to the cylinder does an excellent job reproducing scattering levels and effects. 
Figure 4.10. Analytical (4.10a.) and finite difference (PULSE5 in 4.10b. and PULSE10 in 4.10 c.) pressure time series vs. azimuth for the transient source problem. The finite difference results contain two numerical features which do not appear in the analytical solution. These are the return from the axis of symmetry (curved arrival in the upper left hand portion of figure 4.10b. and 4.10c.) and the direct source arrival (strong arrivals in the lower right and left hand portions of $4.10 \mathrm{~b}$. and $4.10 \mathrm{c}$.). 


\section{Pressure time series}
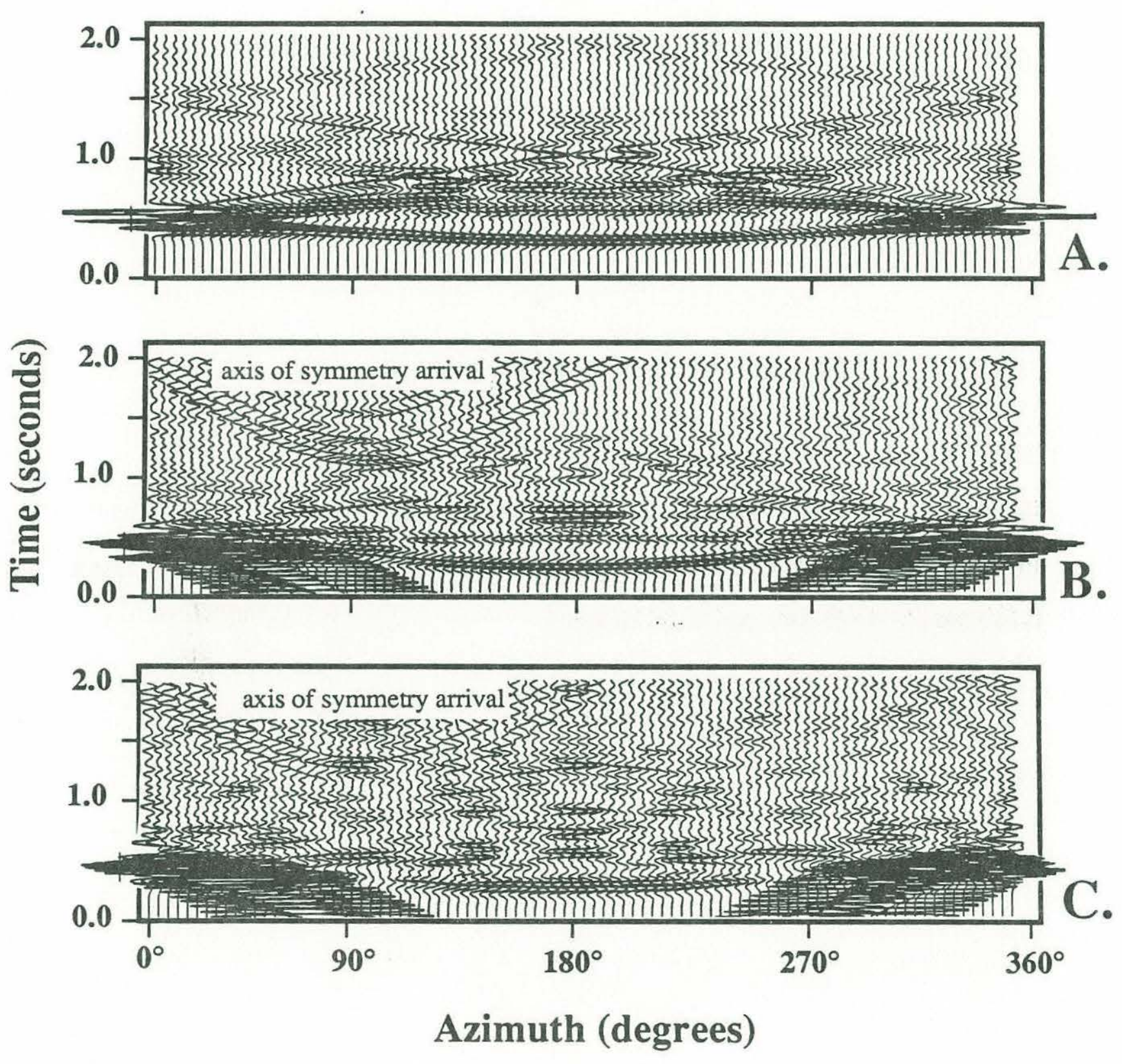
Figure 4.11. Backscatter form function for analytical results (solid line) and model

PULSE5 (dashed line). Cylinder radii used to plot PULSE5 results (see equation 21) is a. $0.160 \mathrm{~km}$. , b. $0.165 \mathrm{~km}$. , and c. $0.170 \mathrm{~km}$. 


\section{BACKSCATTER FORM FUNCTION}

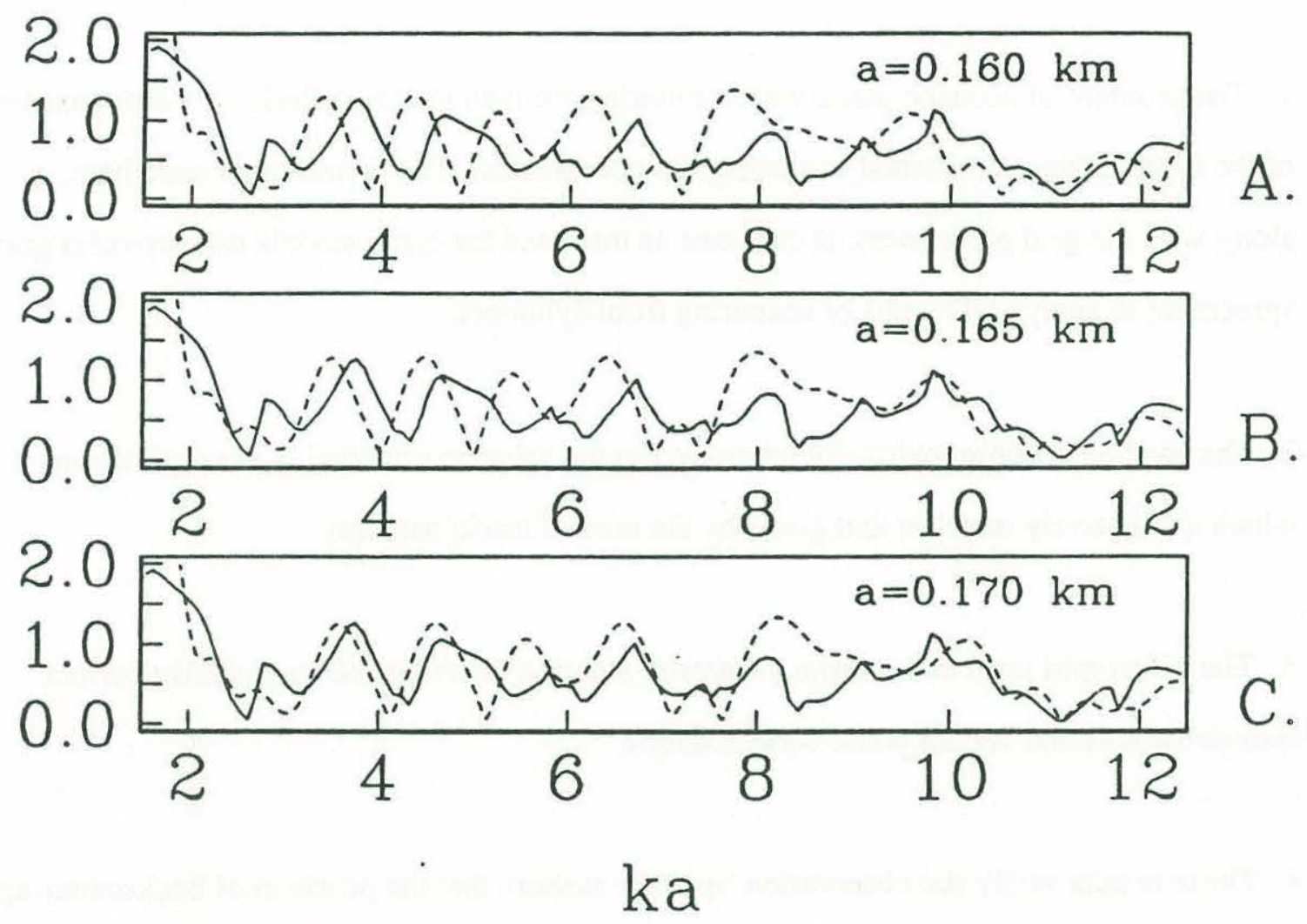

ANALYTICAL -
FIN. DIFF. 


\section{CONCLUSIONS}

1. The problem of acoustic plane wave scattering from an elastic cylinder is a stringent test of the finite difference method in rectangular coordinates. The formulation used here, along with the grid parameters, is the same as that used for earth models and provides good agreement to analytical results of scattering from cylinders.

2. The method is computationally intensive but the solution obtained is a complete one which qualitatively matches that given by the normal mode solution.

3. The offset grid used in the finite difference solution provides the analytically correct symmetric solution for the plane wave sources.

4. These results verify the observation by other authors that the problem of backscattering from a cylinder is very sensitive to cylinder diameter (or conversely, to frequency). The finite difference solution appears to be seeing a slightly larger cylinder that that actually defined on the grid.

5. Rough corners caused by the definition of the curved cylinder boundary on the square finite difference grid complicate the solution. Decreasing the size of the grid step lessened this problem for the pulse source models but finer grids are probably necessary for an 'exact' match to the analytical solution. This problem is heightened by the magnitude of the velocity contrast at the cylinder boundary and is probably not as important for the earth models with much smaller velocity heterogeneity contrasts and gradational (rather that sharp) scatterer boundaries. 


\section{REFERENCES}

Alterman, Z. and Loewenthal, D., (1972), Computer generated seismograms, Methods in Computational Physics, New York, Academic Press.

Brill, D. and Überall, H., (1970), Acoustic waves transmitted through solid elastic cylinders, J. Acoust. Soc. Am., 50(2), 921-939.

Dardy, H. D., Bucard, J.A., Schuetz, L.S., and Dragonette, L.R., (1977), Dynamic widebandwidth acoustic form function determination, J. Acoust. Soc. Am., 62(6), 1373-1376.

Doolittle, R. D. and Überall, H., (1966), Sound scattering by elastic cylindrical shells, J. Acoust. Soc. Am., 39(2), 272-275.

Doolittle, R. D., Überall, H., and Ugincius, P., 1968, Sound scattering by elastic cylinders, J. Acoust. Soc. Am., 43(1), 1-14.

Dougherty, M. E. and Stephen, R. A., (1988), Seismic energy partitioning and scattering $i$ n laterally heterogeneous ocean crust, PAGEOPH, 128(1/2), 195-229.

Faran, J. J., (1951), Sound scattering by solid cylinders and spheres, J. Acoust. Soc. Am., 23, 405-418.

Frankel, A. and Clayton, R. W., (1984), A finite difference simulation of wave propagation in two-dimensional random media, Bull. Seis. Soc. Am., 74, 21672186.

Frisk, G. V., Dickey, J.W., and Überall, H., (1975), Surface wave modes on elastic cylinders, J. Acoust. Soc. Am., 58(5), 996-1008.

Grace, O. D. and Goodman, R. R., (1966), Circumferential waves on solid cylinders, J. Acoust. Soc. Am., 39, 173-174.

Harbold, M. L. and Steinberg, B. N., (1968), Direct experimental verification of creeping waves, J. Acoust. Soc. Am., 45(3), 592-603. 
Karal, F. C. and Keller, J. B., (1964), Elastic, electromagnetic, and other waves in a random medium, J. Math. Phys., 5, 527-547.

Kelly, K. R., Ward, R.W., Treitel, Sven, and Alford, R.M., (1976), Synthetic seismograms: A finite difference approach, Geophysics, 41, 2-27.

Levander, A. R. and Hill, N. R., (1985), P-SV resonances in irregular low velocity surface layers, Bull. Seis. Soc. Am., 75, 847--864.

Lowan, A. N., Morse, P.M., Feshbach, H., and Lax, M., (1946), Scattering and Radiation from Circular Cylinders and Spheres, Mathematical Tables Project and M.I.T. Underwater Sound Laboratory, Washington, D.C., U.S. Navy Department, Office of Research and Inventions.

Neubauer, W. G., (1968), Pulsed circumferential waves on aluminium cylinders in water, J. Acoust. Soc. Am., 45(5), 1134-1144.

Neubauer, W. G., (1986), Acoustic Reflection from surfaces and shapes, Washington, D.C., Naval Research Laboratory.

Neubauer, W. G., Voyt, R.H., and Dragonette, L.R., (1974a), Acoustic reflection from elastic spheres and rigid spheres and spheroids II. Transient analysis,, J. Acoust. Soc. Am., 55(6), 1130-1137.

Neubauer, W. G., Voyt, R.H., and Dragonette, L.R., (1974b), Acoustic reflection from elastic spheres I. Steady state signals, J. Acoust. Soc. Am., 55(6), 1123-1129.

Rayleigh, L., (1878), The Theory of Sound, London, Macmillan.

Stephen, R. A., (1983), A comparison of finite difference and reflectivity seismograms for marine models, J. R. astr. Soc., 72, 39-58.

Stephen, R. A., (1985), Finite-difference synthetic acoustic logs, 50(10), 1588-1609.

Stephen, R. A.,1988,Finite difference methods for bottom interaction problems,Computational Acoustics: Wave Propagation,D. Lee, R. L. Sternberg and M. H. Schultz,225-238. 
Stoyanov, A., Überall, H., Luppé, F., and Quentin, G., (1989), Observation of surface waves on a solid cylinder in a fluid, J. Acoust. Soc. Am., 85(1), 137-140.

Virieux, J., (1984), SH-wave propagation in heterogeneous media: Velocity-stress finitedifference method, Geophysics, 49, 1933-1957.

Virieux, J., (1986), P-SV wave propagation in heterogeneous media: Velocity-stress finite difference method, Geophysics, 51(4), 889-901.

Watson, G. N., (1919), Proc. Roy. Soc. (London), A95, 83-99. 


\section{CONCLUSIONS}




\section{Conclusions}

The subject of this work is the scattering of seismo/acoustic energy from lateral heterogeneities in the upper oceanic crust. For simplicity, most seismic studies in the past have assumed lateral homogeneity even though obvious scattering effects are seen in almost all seismic field data. Another common theme throughout the chapters of this thesis is that the scatterers studied all have spatial dimensions on the same order of magnitude as the seismo/acoustic wavelength. In this realm of scattering problems, scattering reaches a maximum and full wave, laterally heterogeneous modeling schemes are essential. The finite difference method of calculating wave propagation through heterogeneous elastic media was used for the various studies comprising this work. Results from the modeling demonstrate the importance of considering lateral heterogeneity as a scattering mechanism. Also, the work of chapters three and four point out some restrictions of the method for use with the rough seafloor scattering problem. A brief summary of the results from the individual chapters is presented here.

Chapter one dealt with the modeling of a diffracted arrival seen in a particular line of ocean bottom hydrophone data from the Rivera Ocean Seismic Experiment. This arrival is referred to as a 'refraction branch diffraction' because of its location in time and space after the refracted arrival and to distinguish it from a near normal incidence diffraction hyperbola. Finite difference models of deterministic seafloor features demonstrated that this arrival could be due to diffraction from any of a number of topographic features in the area. Energy scattered from the seafloor scatterer travels through the crust back to the ocean bottom receiver. Other arrivals, such as converted shear waves, interference waves and the pseudo-Rayleigh wave occur in the models but not in the original field data. This is probably due to the use of an inappropriate Poisson's ratio 
for the area (no shear conversion for higher Poisson's ratio) and the presence of more complicated topography in the field area (which would attenuate the interference waves).

The problem of incoherent scattering from continuous randomly heterogeneous upper oceanic crust was investigated in chapter two. Although these models dealt with flat seafloors and the strength of the heterogeneity may be debatable, this type of crust is probably much more realistic that a strictly laterally homogeneous one. For random velocity perturbations with a Gaussian distribution, there is a general trend of increasing random scatter as $k a$ ( $\mathrm{k}=$ wavenumber, $\mathrm{a}=$ correlation length) approaches one. Secondary deterministic scattering from the heterogeneities increases as $k a$ increases above one (larger heterogeneities). Self-similar distributions cause scattering with characteristics of a range of $k a$ values.

Heterogeneities in the models presented in chapter two act as secondary sources for Stoneley waves along the water-solid interface. This mechanism may be one explanation for the generation of seafloor noise which may also propagate partly in the form of interface waves. The final major result from chapter two is that propagation through continuously heterogeneous crust affects the correlation of primary arrival waveforms. In general, decorrelation reaches a maximum as $k a$ approaches one.

The effects of scattering from seafloor topography can be very similar to those from lateral heterogeneities. The purpose of the work in chapter 3 was to investigate scattering from more complicated topographic profiles than the isolated deterministic scatterers of chapter one. A more important result from chapter 3 was the evaluation of the use of the finite difference method with a rectangular grid for rough seafloor problems. The problems encountered in using this method involve the discretization of a curved interface on a rectangular grid. There is significant scattering of energy from the stair-step 
microroughness superimposed on the larger scale topography. Although this microroughness should not be present to solve the sinusoidal seafloor problem, the relatively large amount of energy scattered from these small features (1/15 wavelength) does point out the need to consider small scale topography in the scattering problem. Because some of this energy also travels as interface waves, this could be another mechanism for the generation of seafloor noise.

Despite the problem of scattering from the microroughness, many 'real' effects of propagation of the sinusoidal topography were also observed in the models. Travel time anomalies, multiple compressional and shear diving waves, as well as some strong backscattered arrivals are due to the larger scale topography. Steep topography allows energy, especially shear energy, to enter the seafloor even at great ranges. Ray theoretical shadow zones are not present because of Franz-type waves diffracted into areas where the grazing angle is less than zero.

Chapter 4 involved the use of the time domain finite difference method to solve the problem of acoustic wave scattering from an infinite elastic cylinder. Scattering mechanisms and grid considerations seen in this chapter are closely tied to those seen in the results of chapter three. The same finite difference formulation and grid used in chapters two and three was used unmodified for the cylinder problem in chapter 4 . This formulation provided good agreement to the analytical solution of the problem. The results obtained here agree with the observations of other authors that this problem is very sensitive to cylinder diameter. The finite difference solution appears to be seeing a slightly larger cylinder than that actually defined on the grid.

Discretization of the round cylinder cross-section on the rectangular finite difference grid caused the same type of problems as seen in chapter three with sloping seafloors. 
These problems were lessened somewhat in the models of both chapters by decreasing the grid step size but an even finer grid would be necessary to more accurately represent smoothly varying interfaces. The magnitude of the velocity contrast between the solid and the surrounding acoustic medium heightens this problem of scattering from rough corners (caused by the discretization). This is probably not an issue for the earth models in chapter two with dramatically smaller velocity contrasts and gradational scatterer boundaries.

An important point brought out by the results of chapters three and four is that the complexity of the model must be matched to the appropriate modeling technique. The 'simple' models presented in these chapters are probably better solved using deformed grids, different coordinate systems, or analytical methods. However, the objective of this work was to evaluate the use of the finite difference method with a rectangular grid for rough seafloor problems. It was found that extremely fine grids must be used in order to accurately represent the smoothly varying curved interfaces of the sinusoidal seafloor and cylinder models. The power of the finite difference method lies in its ability to handle models with media too complex to be solved analytically. The models in this study are a starting point for more complex models and help to point out some of the restrictions of the method. If the specification of the medium must be at a very fine scale for detailed earth structure, then a very fine finite difference grid is necessary and appropriate. The finite difference method using a rectangular grid will be an important tool for determining just how detailed the seafloor must be sampled deterministically to accurately represent interaction with a seismo/acoustic wavefield. It may turn out that interaction with smaller features (with respect to energy wavelength) can be represented as a stochastic component superimposed on the deterministic effects of larger scale topography. 
Common themes occur throughout the chapters of this work. First, since the sizes of the heterogeneities in all of the models have at least one dimension which is on the order of magnitude of the seismic wavelength, scattering effects are strong and concern the wavelike characteristics of the energy. Scattering effects from lateral volume heterogeneities and small topographic features (such as the grid-imposed steps of chapters three and four) are similar and both types of heterogeneities can act as secondary sources for Stoneley waves along the water-solid interface. Deterministic effects such as travel time and amplitude anomalies are stronger for the rough seafloor models because of the sharp impedance contrast and size of the large scale topography. These effects would be less for sediment covered or 'softer' seafloors and smaller topographic features. Franz waves and the lack of shadow zones due to the interaction of acoustic waves with the curved interface of a cylinder can also be seen in the sinusoidal seafloor models.

The work presented here ties in well with current areas of interest in marine seismology. There is much interest presently in the generation and propagation of seafloor noise. It has been shown here that scattering from topographic and volume heterogeneities can be an important mechanism for coupling of body waves (both in the water and in the upper crust) into interface waves along the seafloor. Areas of future work in this area include determining the relative importance of volume vs. surface scattering mechanisms and the influence of bottom elastic parameters on the generation of secondary Stoneley waves. Closely spaced array experiments should be able to identify modes of propagation of the noise.

Also of current interest is the fine scale characterization of the upper oceanic crust. The models shown here lead to the hypothesis that there is information regarding the fine scale structure of the oceanic crust to be found in the secondary features of seismograms. Inversion of seismic data for heterogeneity length scales should be possible after further 
evaluation of model results such as those presented here. This may, however, involve the collection of seismic data with very closely spaced receivers in order to properly identify phases. The ultimate goal of obtaining knowledge of fine scale crustal structure is to understand mechanisms of crustal formation at the mid-ocean ridges and the processes of evolution of the crust with age.

Reverberation of energy from the seafloor is another area of research activity where these results are relevant. Not only have the models in chapter three shown the importance of small scale (with respect to wavelength) topographic features in the scattering problem, they have also demonstrated an important mechanism for allowing energy to enter the upper crust at large ranges (beyond the flat seafloor critical range). It is currently not understood whether 'reverberated' energy is due to scattering from seafloor topography or volume heterogeneities. The type of modeling done here will help to determine the importance of considering elastic parameters of the bottom as well as seafloor topography in the acoustic reverberation problem. Computer resources are becoming available which will allow very fine grids to be used in rough seafloor models. These grids will be used to determine the influence of fine scale topography on the scattered field, that is, whether or not the small scale interactions must be modelled deterministically or whether they can be included as a stochastic component superimposed on larger scale deterministic effects. 